\title{
Prehospital Airway Management: A Systematic Review
}




\section{Comparative Effectiveness Review}

Number 243

\section{Prehospital Airway Management: A Systematic Review}

Prepared for:

Agency for Healthcare Research and Quality

U.S. Department of Health and Human Services

5600 Fishers Lane

Rockville, MD 20857

www.ahrq.gov

Contract No. 290-2015-00009-I

Prepared by:

Pacific Northwest Evidence-based Practice Center

Portland, OR

Investigators:

Nancy Carney, Ph.D.

Tamara Cheney, M.D.

Annette M. Totten, Ph.D.

Rebecca Jungbauer, Dr.P.H.

Matthew R. Neth, M.D.

Chandler Weeks, M.P.H.

Cynthia Davis-O'Reilly, B.S.

Rochelle Fu, Ph.D.

Yun Yu, M.S.

Roger Chou, M.D.

Mohamud Daya, M.D., M.S.

AHRQ Publication No. 21-EHC023

June 2021 
This report is based on research conducted by the Pacific Northwest Evidence-based Practice Center (EPC) under contract to the Agency for Healthcare Research and Quality (AHRQ), Rockville, MD (Contract No. 290-2015-00009-I) with funding provided by the Office of Emergency Medical Services (OEMS) within the National Highway Traffic Safety Administration (NHTSA). The findings and conclusions in this document are those of the authors, who are responsible for its contents; the findings and conclusions do not necessarily represent the views of AHRQ. Therefore, no statement in this report should be construed as an official position of AHRQ, the U.S. Department of Health and Human Services, or OEMS, NHTSA, U.S Department of Transportation.

\section{None of the investigators have any affiliations or financial involvement that conflicts with the material presented in this report.}

The information in this report is intended to help healthcare decision makers - patients and clinicians, health system leaders, and policymakers, among others-make well-informed decisions and thereby improve the quality of healthcare services. This report is not intended to be a substitute for the application of clinical judgment. Anyone who makes decisions concerning the provision of clinical care should consider this report in the same way as any medical reference and in conjunction with all other pertinent information, i.e., in the context of available resources and circumstances presented by individual patients.

This report is made available to the public under the terms of a licensing agreement between the author and the Agency for Healthcare Research and Quality. This report may be used and reprinted without permission except those copyrighted materials that are clearly noted in the report. Further reproduction of those copyrighted materials is prohibited without the express permission of copyright holders.

AHRQ or U.S. Department of Health and Human Services endorsement of any derivative products that may be developed from this report, such as clinical practice guidelines, other quality enhancement tools, or reimbursement or coverage policies, may not be stated or implied.

AHRQ appreciates appropriate acknowledgment and citation of its work. Suggested language for acknowledgment: This work was based on an evidence report, Prehospital Airway Management: A Systematic Review, by the Evidence-based Practice Center Program at the Agency for Healthcare Research and Quality (AHRQ).

Suggested citation: Carney N, Cheney T, Totten AM, Jungbauer R, Neth MR, Weeks C, DavisO’Reilly C, Fu R, Yu Y, Chou R, Daya M. Prehospital Airway Management: A Systematic Review. Comparative Effectiveness Review No. 243. (Prepared by the Pacific Northwest Evidence-based Practice Center under Contract No. 290-2015-00009-I.) AHRQ Publication No. 21-EHC023. Rockville, MD: Agency for Healthcare Research and Quality; June 2021. DOI: https://doi.org/10.23970/AHRQEPCCER243. Posted final reports are located on the Effective Health Care Program search page. 


\section{Preface}

The Agency for Healthcare Research and Quality (AHRQ), through its Evidence-based Practice Centers (EPCs), sponsors the development of evidence reports and technology assessments to assist public- and private-sector organizations in their efforts to improve the quality of healthcare in the United States.

The National Highway Traffic Safety Administration Office of Emergency Medical Services requested and funded this report from the EPC Program at AHRQ. AHRQ assigned this report to the following EPC: Pacific Northwest Evidence-based Practice Center (Contract No. 290-201500009-I).

The reports and assessments provide organizations with comprehensive, evidence-based information on common medical conditions and new healthcare technologies and strategies. They also identify research gaps in the selected scientific area, identify methodological and scientific weaknesses, suggest research needs, and move the field forward through an unbiased, evidence-based assessment of the available literature. The EPCs systematically review the relevant scientific literature on topics assigned to them by AHRQ and conduct additional analyses when appropriate prior to developing their reports and assessments.

To bring the broadest range of experts into the development of evidence reports and health technology assessments, AHRQ encourages the EPCs to form partnerships and enter into collaborations with other medical and research organizations. The EPCs work with these partner organizations to ensure that the evidence reports and technology assessments they produce will become building blocks for healthcare quality improvement projects throughout the Nation. The reports undergo peer review and public comment prior to their release as a final report.

AHRQ expects that the EPC evidence reports and technology assessments, when appropriate, will inform individual health plans, providers, and purchasers as well as the healthcare system as a whole by providing important information to help improve healthcare quality.

If you have comments on this evidence report, they may be sent by mail to the Task Order Officer named below at: Agency for Healthcare Research and Quality, 5600 Fishers Lane, Rockville, MD 20857, or by email to epc@ahrq.hhs.gov.

David Meyers, M.D.

Acting Director

Agency for Healthcare Research and Quality

Christine Chang, M.D., M.P.H.

Acting Director

Evidence-based Practice Center Program

Center for Evidence and Practice Improvement Agency for Healthcare Research and Quality
Arlene S. Bierman, M.D., M.S.

Director

Center for Evidence and Practice

Improvement

Agency for Healthcare Research and Quality

David W. Niebuhr, M.D., M.P.H., M.Sc.

Task Order Officer

Center for Evidence and Practice

Improvement

Agency for Healthcare Research and Quality 


\section{Acknowledgments}

The authors gratefully acknowledge the following individuals for their contributions to this project: Tracy Dana, M.L.S., Leah Williams, B.S., and Elaine Graham, M.L.S.

\section{Key Informants}

In designing the study questions, the EPC consulted several Key Informants who represent the end-users of research. The EPC sought the Key Informant input on the priority areas for research and synthesis. Key Informants are not involved in the analysis of the evidence or the writing of the report. Therefore, in the end, study questions, design, methodological approaches, and/or conclusions do not necessarily represent the views of individual Key Informants.

Key Informants must disclose any financial conflicts of interest greater than $\$ 5,000$ and any other relevant business or professional conflicts of interest. Because of their role as end-users, individuals with potential conflicts may be retained. The TOO and the EPC work to balance, manage, or mitigate any conflicts of interest.

The list of Key Informants who provided input to this report follows:

Jeremy Brown, M.D.

Director, NIH Office of Emergency Care

Bethesda, MD

Kathleen Brown, M.D.

Associate Division Chief for Clinical Affairs

Medical Director, Emergency Medicine

Children's National Health System

Washington, D.C.

Michael Davis, M.D., FACS

Colonel, USAF, Department of Defense

Director, U.S. Army Medical Research

Fort Detrick, MD

John Gallagher, M.D.

Director, Wichita/Sedgewick County EMS

System

Wichita, KS

Marianne Gausche-Hill, M.D., FACEP, FAAP, FAEMS

Medical Director, Los Angeles County

Emergency Medical Services

Professor of Clinical Emergency Medicine

and Pediatrics

UCLA

Torrance, CA
Matt Hansen, M.D., M.C.R.

Associate Professor of Emergency Medicine

Oregon Health \& Science University

Portland, OR

Rick Hunt, M.D., FACEP

Senior Medical Advisor

National Health Care Preparedness

Programs, ASPR (HHS)

Washington, DC

Jamie Kennel, M.S., NREMT-P

Program Director and Associate Professor

Oregon Paramedic Education Program

Klamath Falls, OR

E. Brooke Lerner, Ph.D., FAEMS

Emergency Medicine Jacobs School of

Medicine and Biomedical Sciences

Buffalo, NY

Michael Levy, M.D., FACEP, FAAEM,

FACP

National Association of EMS Officials

Anchorage, AK 
Ashish Panchal, M.D.

National Registry of EMTs

Emergency Medicine Ohio State University

Columbus, $\mathrm{OH}$

Diane Pilkey, R.N., M.P.H, HHS/HRSA/EMS-C

Emergency Medical Services for Children

Health Resources Services Administration,

HHS

Rockville, MD
Henry Wang, M.D., M.S.

Professor and Executive Vice-Chair of

Research

Department of Emergency Medicine

The Ohio State University

Columbus, $\mathrm{OH}$

\section{Technical Expert Panel}

In designing the study questions and methodology at the outset of this report, the EPC consulted several technical and content experts. Broad expertise and perspectives were sought. Divergent and conflicted opinions are common and perceived as healthy scientific discourse that results in a thoughtful, relevant systematic review. Therefore, in the end, study questions, design, methodologic approaches, and/or conclusions do not necessarily represent the views of individual technical and content experts.

Technical Experts must disclose any financial conflicts of interest greater than $\$ 5,000$ and any other relevant business or professional conflicts of interest. Because of their unique clinical or content expertise, individuals with potential conflicts may be retained. The TOO and the EPC work to balance, manage, or mitigate any potential conflicts of interest identified.

The list of Technical Experts who provided input to this report follows:

Kathleen Adelgais, M.D., M.P.H./M.S.P.H.*

Professor, Pediatrics-Emergency Medicine

Children's Hospital Colorado

University of Colorado

Denver, CO

Michael Aziz, M.D.*

Professor Anesthesiology and Perioperative

Medicine

Interim Vice Chair for Clinical Affairs

Anesthesiology and Perioperative Medicine

Oregon Health \& Science University

Portland, OR

Justin Benoit, M.D., M.S., FAEMS*

Assistant Professor of Emergency Medicine

University of Cincinnati

Cincinnati, $\mathrm{OH}$
Jeffrey M. Elder, M.D., FAAEM, FAEMS*

Medical Director of Emergency

Management for Louisiana Children's

Medical Center Health

Clinical Associate Professor in Emergency

Medicine

Louisiana State University Emergency

Medicine

New Orleans, LA

Tony Fernandez, Ph.D., NREMT-P

Research Assistant Professor

EMPPIC Director of Research

EMS Performance Improvement Center

University of North Carolina

Chapel Hill, NC 
Francis X. Guyette, M.D., M.S., M.P.H., FACEP, FAEMS*

Associate Professor of Emergency Medicine

Medical Director, STAT MedEvac

Emergency Department Attending Physician

University of Pittsburgh

Pittsburgh, PA
Jeffrey L. Jarvis, M.D., M.S., EMT-P*

Medical Director for Williamson County

EMS and Marble Falls Area EMS

Georgetown, TX

Henry Wang, M.D., M.S.*

Professor and Executive Vice-Chair of

Research

Department of Emergency Medicine

The Ohio State University

Columbus, $\mathrm{OH}$

*Provided input on Draft Report.

\section{Peer Reviewers}

Prior to publication of the final evidence report, EPCs sought input from independent Peer Reviewers without financial conflicts of interest. However, the conclusions and synthesis of the scientific literature presented in this report do not necessarily represent the views of individual reviewers.

Peer Reviewers must disclose any financial conflicts of interest greater than $\$ 5,000$ and any other relevant business or professional conflicts of interest. Because of their unique clinical or content expertise, individuals with potential nonfinancial conflicts may be retained. The TOO and the EPC work to balance, manage, or mitigate any potential nonfinancial conflicts of interest identified.

The list of Peer Reviewers follows:

Nichole Bosson, M.D., M.P.H., FAEMS

Assistant Medical Director, Los Angeles County EMS Agency Department of Emergency Medicine, Harbor-UCLA Medical Center

Los Angeles, CA

Darren Braude, M.D., EMT-P

Professor of Emergency Medicine and

Anesthesiology

University of New Mexico

Albuquerque, NM
Craig Goolsby, M.D., M.Ed., FACEP

Department of Military and Emergency

Medicine

National Center for Disaster Medicine and Public Health

Uniformed Services University of the Health

Sciences

Bethesda, MD 


\section{Prehospital Airway Management: A Systematic Review}

\section{Structured Abstract}

Objective. To assess the comparative benefits and harms across three airway management approaches (bag valve mask [BVM], supraglottic airway [SGA], and endotracheal intubation [ETI]) by emergency medical services in the prehospital setting, and how the benefits and harms differ based on patient characteristics, techniques, and devices.

Data sources. We searched electronic citation databases $\left(\right.$ Ovid $^{\circledR}$ MEDLINE $^{\circledR}, \mathrm{CINAHL}^{\circledR}$, the Cochrane Central Register of Controlled Trials, the Cochrane Database of Systematic Reviews, and Scopus ${ }^{\circledR}$ ) from 1990 to September 2020 and reference lists, and posted a Federal Register notice request for data.

Review methods. Review methods followed Agency for Healthcare Research and Quality Evidence-based Practice Center Program methods guidance. Using pre-established criteria, studies were selected and dual reviewed, data were abstracted, and studies were evaluated for risk of bias. Meta-analyses using profile-likelihood random effects models were conducted when data were available from studies reporting on similar outcomes, with analyses stratified by study design, emergency type, and age. We qualitatively synthesized results when meta-analysis was not indicated. Strength of evidence (SOE) was assessed for primary outcomes (survival, neurological function, return of spontaneous circulation [ROSC], and successful advanced airway insertion [for SGA and ETI only]).

Results. We included 99 studies (22 randomized controlled trials and 77 observational studies) involving 630,397 patients. Overall, we found few differences in primary outcomes when airway management approaches were compared.

- For survival, there was moderate SOE for findings of no difference for BVM versus ETI in adult and mixed-age cardiac arrest patients. There was low SOE for no difference in these patients for BVM versus SGA and SGA versus ETI. There was low SOE for all three comparisons in pediatric cardiac arrest patients, and low SOE in adult trauma patients when BVM was compared with ETI.

- For neurological function, there was moderate SOE for no difference for BVM compared with ETI in adults with cardiac arrest. There was low SOE for no difference in pediatric cardiac arrest for BVM versus ETI and SGA versus ETI. In adults with cardiac arrest, neurological function was better for BVM and ETI compared with SGA (both low SOE).

- ROSC was applicable only in cardiac arrest. For adults, there was low SOE that ROSC was more frequent with SGA compared with ETI, and no difference for BVM versus SGA or BVM versus ETI. In pediatric patients there was low SOE of no difference for BVM versus ETI and SGA versus ETI.

- For successful advanced airway insertion, low SOE supported better first-pass success with SGA in adult and pediatric cardiac arrest patients and adult patients in studies that mixed emergency types. Low SOE also supported no difference for first-pass success in 
adult medical patients. For overall success, there was moderate SOE of no difference for adults with cardiac arrest, medical, and mixed emergency types.

- While harms were not always measured or reported, moderate SOE supported all available findings. There were no differences in harms for BVM versus SGA or ETI. When SGA was compared with ETI, there were no differences for aspiration, oral/airway trauma, and regurgitation; SGA was better for multiple insertion attempts; and ETI was better for inadequate ventilation.

Conclusions. The most common findings, across emergency types and age groups, were of no differences in primary outcomes when prehospital airway management approaches were compared. As most of the included studies were observational, these findings may reflect study design and methodological limitations. Due to the dynamic nature of the prehospital environment, the results are susceptible to indication and survival biases as well as confounding; however, the current evidence does not favor more invasive airway approaches. No conclusion was supported by high SOE for any comparison and patient group. This supports the need for high-quality randomized controlled trials designed to account for the variability and dynamic nature of prehospital airway management to advance and inform clinical practice as well as emergency medical services education and policy, and to improve patient-centered outcomes. 


\section{Contents}

Evidence Summary .......................................................................................................................... ES-1

Introduction ............................................................................................................................. 1

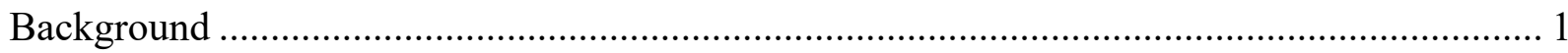

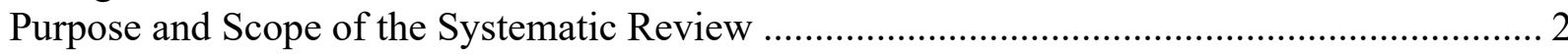

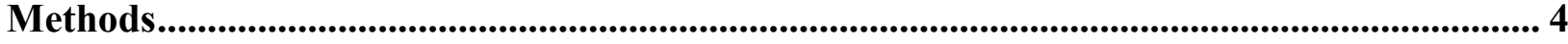

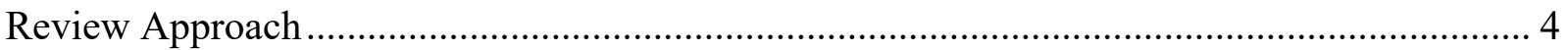

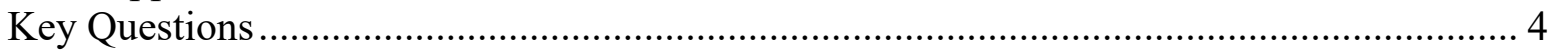

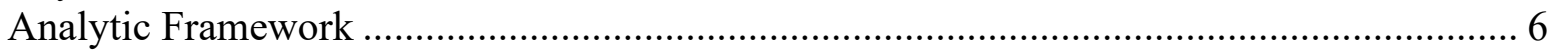

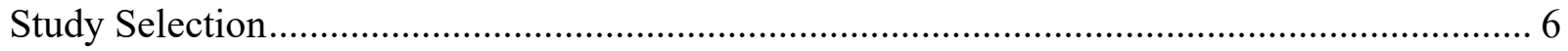

Data Extraction and Risk of Bias Assessment ………….................................................... 7

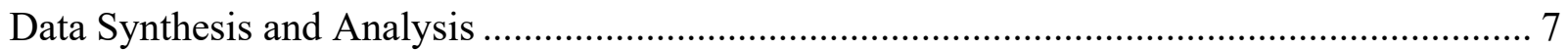

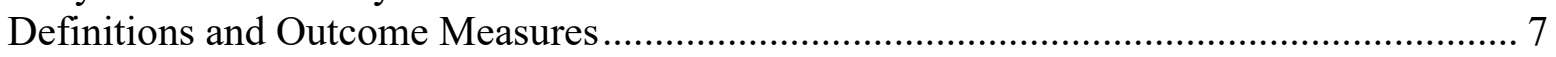

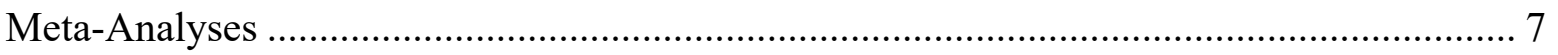

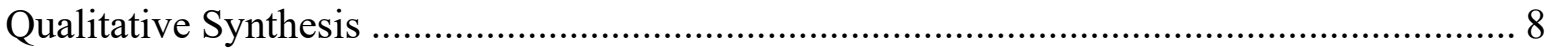

Grading the Strength of the Body of Evidence ………...................................................... 8

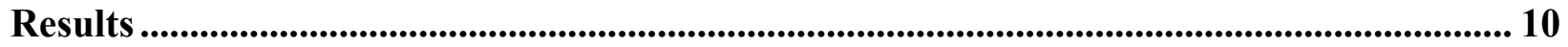

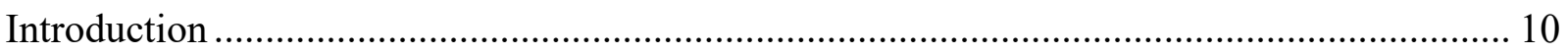

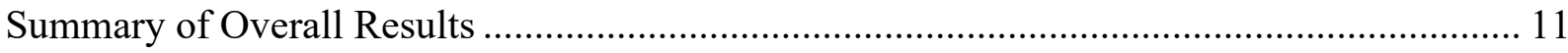

Individual Key Question Summaries …………………………………………………..... 15

Key Question 1: Bag Valve Mask Compared With Supraglottic Airway .............................. 15

Key Question 2: Bag Valve Mask Compared With Endotracheal Intubation ....................... 18

Key Question 3: Supraglottic Airway Compared With Endotracheal Intubation.................. 22

Key Question 4: Modifiers Within Airway Approaches ................................................ 28

Discussion................................................................................................................................... 46

Findings in Relation to the Decisional Dilemmas................................................................... 46

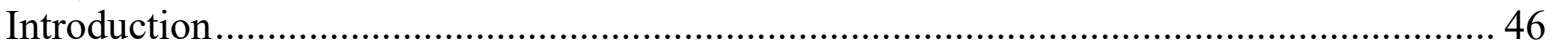

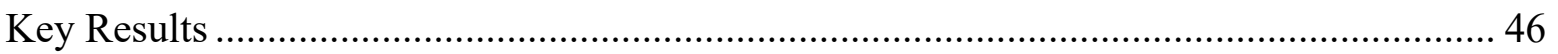

Summary of the Evidence ………….............................................................................. 50

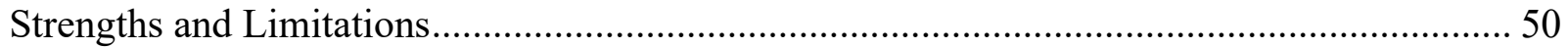

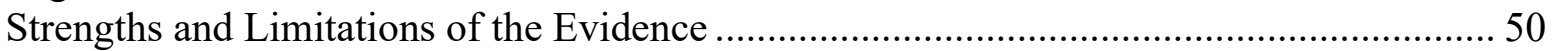

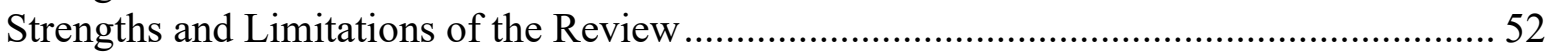

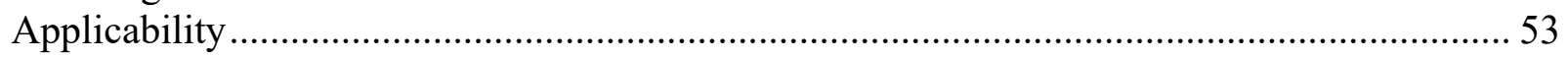

Implications for Clinical Practice, Education, Research, or Health Policy................................ 54

Provider Training, Expertise, and Skills Maintenance ………............................................... 55

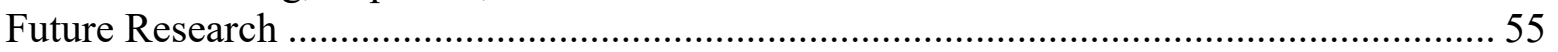

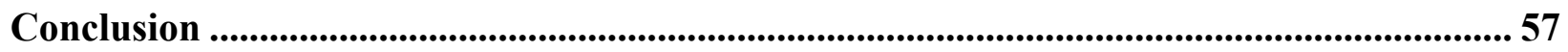

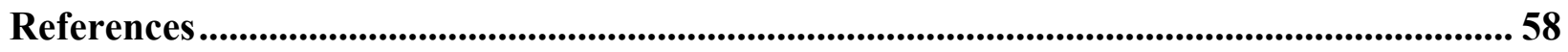

Abbreviations and Acronyms ................................................................................................. 67

\section{Tables}

Table A. Results of airway comparisons by age group ........................................................ ES-4

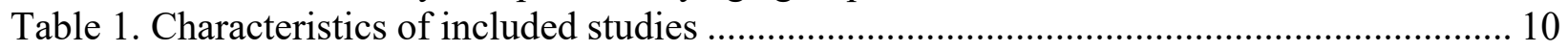

Table 2. Number of studies by Key Question and study design ................................................. 11

Table 3. Overview of conclusions: comparisons by emergency types and age groups ................. 12 
Table 4. Overview of conclusions: successful advanced airway insertion by emergency type and age: Key Question 3 ..................................................................................................... 13

Table 5. Overview of conclusions: outcomes for modifiers of endotracheal intubation: Key

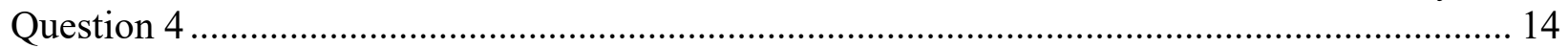

Table 6. SGA versus BVM: survival by emergency type and age group (BVM referent)........... 16

Table 7. SGA versus BVM: neurological function by emergency type and age group (BVM

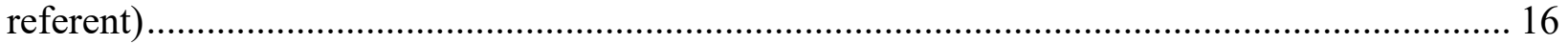

Table 8. SGA versus BVM: ROSC by emergency type and age group (BVM referent) ............. 17

Table 9. BVM versus SGA: additional outcomes............................................................... 17

Table 10. ETI versus BVM: survival in-hospital or at 1-month post incident by emergency type and age groups (BVM referent) ......................................................................................... 19

Table 11. ETI versus BVM: neurological function by emergency types and age groups (BVM

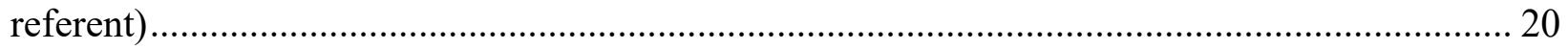

Table 12. ETI versus BVM: ROSC by emergency type and age groups (BVM referent) ............ 20

Table 13. BVM versus ETI: additional outcomes ...................................................................... 21

Table 14. BVM versus ETI: additional studies of survival and neurological function ................. 22

Table 15. ETI versus SGA: survival by emergency type and age group (SGA referent)............. 24

Table 16. ETI versus SGA: neurological function - modified Rankin Scale by age group (SGA

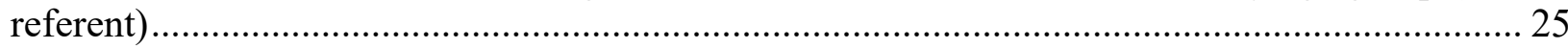

Table 17. ETI versus SGA: neurological function - Cerebral Performance Category / Pediatric

Cerebral Performance Category by age group (SGA referent).................................................. 25

Table 18. ETI versus SGA: ROSC by age group (SGA referent) …………………………..... 25

Table 19. ETI versus SGA: first-pass successful advanced airway insertion by emergency type

and age group (SGA referent) ............................................................................................ 26

Table 20. ETI versus SGA: overall successful advanced airway insertion by emergency type and

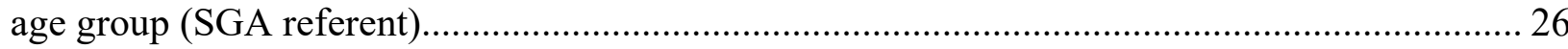

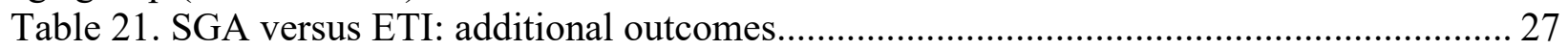

Table 22. Survival for RSI versus no medication by subgroup (no medication referent) ............. 32

Table 23. First-pass success for RSI versus no medication by subgroup (no medication

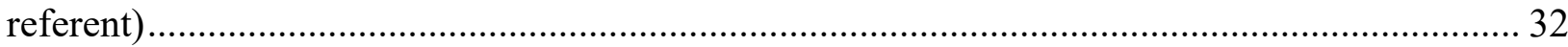

Table 24. Overall success for RSI versus no medication by subgroup (no medication referent). 33

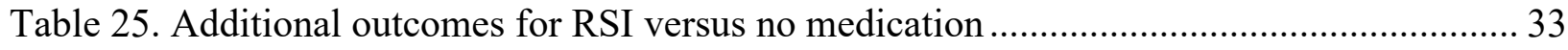

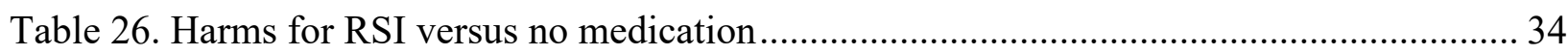

Table 27. First-pass success for RSI versus sedation-facilitated by subgroup (sedation-facilitated

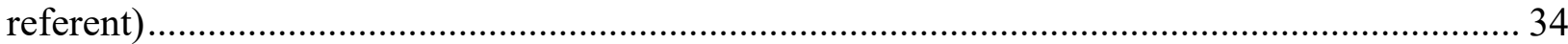

Table 28. Additional outcomes for RSI versus sedation-facilitated ............................................ 35

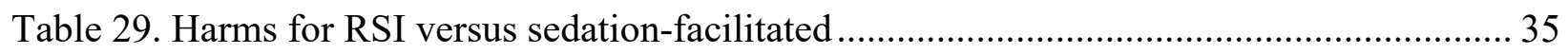

Table 30. First-pass success for sedation-facilitated versus no medication by subgroup (no

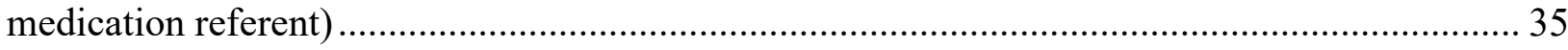

Table 31. Additional outcomes for sedation-facilitated versus no medication............................. 35

Table 32. First-pass success for video versus direct laryngoscopy by subgroup (direct

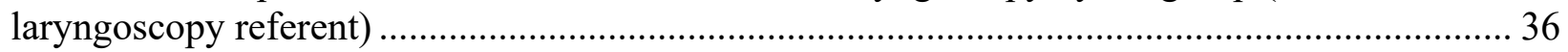

Table 33. Overall success for video versus direct laryngoscopy by subgroup (direct laryngoscopy

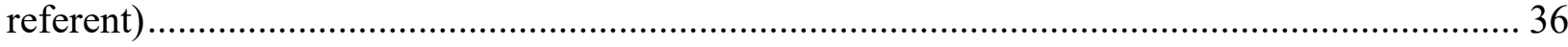

Table 34. Additional outcomes for video versus direct laryngoscopy............................................. 37

Table 35. Harms for video versus direct laryngoscopy ................................................................ 37 
Table 36. Outcomes for comparisons of laryngoscope blades and use of gum elastic bougie..... 38

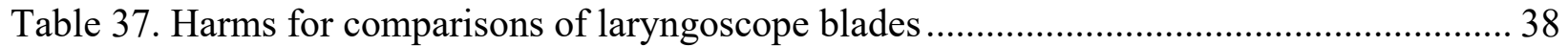

Table 38. First-pass success for comparisons of emergency type ………………….................. 39

Table 39. Overall success for comparisons of emergency type................................................... 39

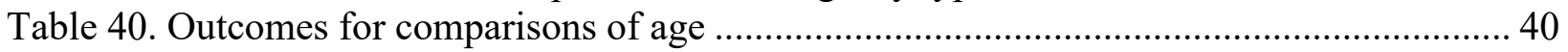

Table 41. Harms for comparisons of age within pediatrics ....................................................... 41

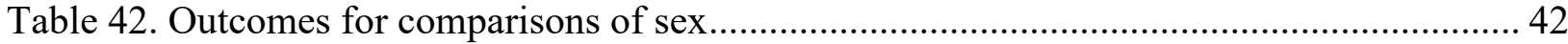

Table 43. Outcomes for comparisons of race ...................................................................... 42

Table 44. Outcomes for comparisons of perilaryngeal seal SGAs ............................................. 43

Table 45. Outcomes for comparisons of pharyngeal versus perilaryngeal seal SGAs .................. 44

Table 46. Harms for comparisons of pharyngeal versus perilaryngeal seal SGAs........................ 44

Table 47. Outcomes for comparisons of pharyngeal seal SGAs .................................................. 45

Table 48. Harms for Combitube versus PTLA …………………………………………….... 45

Table 49. Outcomes for comparisons of age: pediatric versus adult ......................................... 45

Figures

Figure 1. Analytic framework

\section{Appendixes}

Appendix A. Methods

Appendix B. Literature Flow

Appendix C. Included Studies

Appendix D. Excluded Studies

Appendix E. Study Characteristics Evidence Table

Appendix F. Outcomes Evidence Table

Appendix G. Risk of Bias

Appendix H. Meta-Analysis: Primary Analyses

Appendix I. Meta-Analysis: Sensitivity Analysis

Appendix J. Strength of Evidence 


\section{Evidence Summary}

\section{Main Points}

- Four Key Questions addressed the comparative benefits and harms across three airway management approaches by emergency medical services in the prehospital setting: Key Question 1 - bag valve mask [BVM] versus supraglottic airway [SGA]; Key Question 2 BVM versus endotracheal intubation [ETI]); Key Question 3 - SGA versus ETI; and Key Question 4 - how the benefits and harms differ based on patient characteristics, techniques, and devices.

- The most common finding, across emergency types and age groups, was of no differences in primary outcomes when prehospital airway management approaches were directly compared.

- None of the conclusions were supported by high strength of evidence (SOE); thus, future, more rigorous studies could change the findings.

- The following conclusions for Key Questions 1-3 were supported by low or moderate SOE (see Table A):

○ Survival measured in-hospital or at 1-month post incident:

- No difference in outcomes for all three comparisons in adult/mixed-age patients with cardiac arrest and pediatric patients with cardiac arrest.

- No difference when BVM was compared with ETI in adult trauma patients.

- Neurological function measured by the Cerebral Performance Category (CPC), Pediatric CPC, or modified Rankin Scale (mRS) in-hospital or at 1-month post incident:

- When BVM was compared with SGA, outcomes favored BVM in adult patients with cardiac arrest.

- When BVM was compared with ETI, there was no difference in outcomes in adult patients with cardiac arrest.

- When SGA was compared with ETI, outcomes measured by the CPC favored ETI in adult patients with cardiac arrest; there was no difference in outcomes measured by the mRS in this group.

- When ETI was compared with BVM or SGA, there was no difference in outcomes in pediatric patients with cardiac arrest.

- Return of spontaneous circulation (ROSC) (prehospital, sustained, or overall):

- When BVM was compared with SGA or ETI, there was no difference in outcomes in adult patients.

- When SGA was compared with ETI, outcomes favored SGA in adult patients.

- When ETI was compared with BVM or SGA, there was no difference in outcomes in pediatric patients.

- First-pass successful advanced airway insertion (Key Question 3 only):

- When SGA was compared with ETI, outcomes favored SGA in adult and pediatric patients with cardiac arrest and adult patients with mixed emergency types. 
- No difference when SGA was compared with ETI in adult patients with medical emergencies.

- Overall successful advanced airway insertion (Key Question 3 only):

- No difference when SGA was compared with ETI in adult patients with cardiac arrest, medical, or mixed emergency types.

- For other quantitatively analyzed comparisons and outcomes for Key Questions 1-3, there was insufficient evidence to support conclusions.

- Key findings for comparisons within ETI (Key Question 4):

○ Survival measured in hospital:

- No difference when rapid sequence intubation (RSI) was compared to ETI with no medication in adult/mixed-age patients with trauma.

- First-pass successful advanced airway insertion:

- When RSI was compared to ETI with no medication, RSI was favored in adults/mixed-age patients with mixed emergency types; there was no difference in adults/mixed-age patients with trauma.

- No difference when video laryngoscopy was compared with direct laryngoscopy in adult/mixed-age patients with cardiac arrest or mixed emergency types.

- Overall successful advanced airway insertion:

- When RSI was compared to ETI with no medication, RSI was favored in adults with trauma; there was no difference in adults/mixed-age patients with cardiac arrest or mixed emergency types.

- No difference when video laryngoscopy was compared with direct laryngoscopy in adult/mixed-age patients with cardiac arrest or mixed emergency types.

- Implications based on the current body of evidence and finding that no one airway management approach was consistently superior:

- It is possible all three airway management techniques have a role in prehospital care and the preferred airway approach depends on the setting, patient age and type, available provider expertise, and equipment.

○ Future research should:

- Focus on rigorous studies, preferably randomized controlled trials (RCTs), given that important and frequent sources of bias in prehospital airway research are difficult to address in observational studies.

- Construct comparisons that are more clearly defined by specific emergency types, patient groups, and emergency medical service (EMS) resources including training.

\section{Background and Purpose}

Emergency medical services care for people who experience emergencies with the goal of stabilizing, treating, and possibly transporting people to emergency departments. A key component of prehospital care is management of the patient's airway followed by ventilation, which is critical to immediate survival and impacts potential recovery.

Three airway management techniques routinely used by EMS include: BVM, SGA, and ETI. Each requires unique training and equipment. Individual research studies, experience with hospitalized patients, and EMS agency resources and personnel experience have led to questions 
about which prehospital airway management approach is best for what type of patients in specific situations.

Given the complexity of the prehospital environment, many factors are likely to influence patient outcomes, in addition to the airway type. The purpose of this review is to provide a synthesis of the currently available research on the comparative effectiveness of these three airway techniques in prehospital care to help inform EMS practice guidelines and policy.

\section{Methods}

We employed methods consistent with those outlined in the Agency for Healthcare Research and Quality Evidence-based Practice Center Program methods guidance (https://effectivehealthcare.ahrq.gov/topics/cer-methods-guide/overview). We identified and synthesized studies published between January 1, 1990 and September 8, 2020. We included studies that compared two types of airways or compared variations of one type of airway, such as video and direct laryngoscopy. Details about our search strategies, inclusion criteria, assessment, and synthesis of the evidence are included in the full report text and appendices.

Our approach and results were specific to characteristics of airway management and research on this topic. A key characteristic was that in prehospital care there are fundamental differences in airway management requirements for trauma, cardiac arrest, and other medical needs. Similarly, the needs and challenges of airway management for children differ significantly from those for adults. Given these differences, our results were organized into groups defined by age and emergency type. Studies were not combined across these groups, as such combinations would not be clinically meaningful. Pooled estimates were generated separately for RCTs and observational studies; however, the conclusions and SOE assessments presented include all study designs. When there were conflicting findings, we prioritized those from RCTs with low risk of bias.

\section{Results}

Our results synthesized the findings of 99 studies from 101 publications involving 630,397 patients that compared BVM to SGA (Key Question 1, 22 studies), BVM to ETI (Key Question 2, 22 studies), SGA to ETI (Key Question 3, 41 studies), or compared variations of one of the three airway approaches (Key Question 4, 51 studies). The results for Key Questions 1, 2, and 3 for the outcomes of survival in-hospital or at 1-month post incident, neurological function at discharge or at 1-month post incident, ROSC, and first-pass and overall success are presented in Table A.

The overall findings suggested that there are few differences in primary outcomes between the three methods of airway management studied. Similarly, few differences were found in studies that compared variations of one type of airway (e.g., video versus direct laryngoscopy). 
Table A. Overview of conclusions: comparisons by emergency types and age groups

\begin{tabular}{|c|c|c|c|c|}
\hline Outcome & Emergency Type and Age & $\begin{array}{l}\text { KQ1: } \\
\text { BVM vs. SGA }\end{array}$ & $\begin{array}{l}\text { KQ2: } \\
\text { BVM vs. ETI }\end{array}$ & $\begin{array}{l}\text { KQ3: } \\
\text { SGA vs. ETI }\end{array}$ \\
\hline \multirow{4}{*}{ Survival } & $\begin{array}{l}\text { Cardiac arrest: } \\
\text { Adults/Mixed }\end{array}$ & No difference & No difference & No difference \\
\hline & Cardiac arrest: Pediatrics & No difference ${ }^{a}$ & No difference & No difference ${ }^{a}$ \\
\hline & Trauma: Adults & No conclusion ${ }^{a}$ & No difference & No conclusion ${ }^{a}$ \\
\hline & Trauma: Pediatrics & No evidence & No conclusion ${ }^{a}$ & No evidence \\
\hline \multirow{4}{*}{$\begin{array}{l}\text { Neurological } \\
\text { Function }\end{array}$} & Cardiac arrest: Adults & $\begin{array}{l}\text { mRS: No evidence } \\
\text { CPC: Favors BVM }\end{array}$ & $\begin{array}{l}\text { mRS: No evidence } \\
\text { CPC: No } \\
\text { difference }\end{array}$ & $\begin{array}{l}\text { mRS: No difference } \\
\text { CPC: Favors ETI }\end{array}$ \\
\hline & Cardiac arrest: Pediatrics & No conclusion ${ }^{a}$ & No difference & No difference $^{a}$ \\
\hline & Trauma: Adults & No evidence & No evidence & No evidence \\
\hline & Trauma: Pediatrics & No evidence & No evidence & No evidence \\
\hline \multirow[b]{2}{*}{ ROSC $^{b}$} & Cardiac arrest: Adults & No difference & No difference & Favors SGA \\
\hline & Cardiac arrest: Pediatrics & No conclusion ${ }^{a}$ & No difference ${ }^{a}$ & No difference ${ }^{a}$ \\
\hline \multirow{8}{*}{$\begin{array}{l}\text { First-Pass } \\
\text { Success }^{c}\end{array}$} & Cardiac arrest: Adults & $\mathrm{NA}$ & NA & Favors SGA \\
\hline & Cardiac arrest: Pediatrics & $\mathrm{NA}$ & NA & Favors SGA ${ }^{a}$ \\
\hline & Trauma: Adults & NA & NA & No conclusion ${ }^{\mathrm{a}}$ \\
\hline & Trauma: Pediatrics & NA & NA & No conclusion ${ }^{\mathrm{a}}$ \\
\hline & Medical: Adults & NA & NA & No difference \\
\hline & Medical: Pediatrics & NA & NA & No conclusion ${ }^{\mathrm{a}}$ \\
\hline & Mixed: Adults & NA & NA & Favors SGA \\
\hline & Mixed: Pediatrics & $\mathrm{NA}$ & NA & No evidence \\
\hline \multirow{8}{*}{$\begin{array}{l}\text { Overall } \\
\text { Success }^{c}\end{array}$} & Cardiac arrest: Adults & $\mathrm{NA}$ & NA & No difference \\
\hline & Cardiac arrest: Pediatrics & NA & NA & No evidence \\
\hline & Trauma: Adults & $\mathrm{NA}$ & NA & No conclusion ${ }^{\mathrm{a}}$ \\
\hline & Trauma: Pediatrics & NA & NA & No evidence \\
\hline & Medical: Adults & $\mathrm{NA}$ & NA & No difference \\
\hline & Medical: Pediatrics & NA & NA & No evidence \\
\hline & Mixed: Adults & $\mathrm{NA}$ & NA & No difference $^{a}$ \\
\hline & Mixed: Pediatrics & NA & NA & No evidence \\
\hline Harms $^{d}$ & All groups & No difference & No difference & $\begin{array}{l}\text { No difference: } \\
\text { Aspiration, } \\
\text { Oral/Airway Trauma, } \\
\text { Regurgitation } \\
\text { Favors SGA: } \\
\text { Multiple Insertion } \\
\text { Attempts } \\
\text { Favors ETI: } \\
\text { Inadequate } \\
\text { Ventilation }\end{array}$ \\
\hline
\end{tabular}

BVM = bag valve mask; CPC = Cerebral Performance Category; ETI = endotracheal intubation; KQ = Key Question; $\mathrm{mRS}=$ modified Rankin Scale; NA = not applicable; ROSC = return of spontaneous circulation; SGA = supraglottic airway

Bold Text $=$ Moderate SOE, Standard text $=$ Low SOE, Italicized text $=$ Insufficient SOE

${ }^{\text {a }}$ Results based only on observational studies

${ }^{b}$ ROSC was only reported in studies of cardiac arrest

c Success was qualitatively synthesized for KQ1 and 2; results available in full report

${ }^{\mathrm{d}}$ Harms were qualitatively synthesized; meta-analysis not possible as harms are different

Also included in the full report were studies that were analyzed qualitatively, which compared SGA devices, variations on ETI, or reported other outcomes.

\section{Strengths and Limitations}

We identified and pooled studies that compared primary outcomes for different types of airway management used in prehospital care. Given the challenges of this environment, the size of the body of evidence was a key strength. It was also useful that most studies included 
outcomes important to patients and were not limited to process measures that may be less relevant. The most important limitations were weaker observational study designs, rendering them vulnerable to indication and survival biases. Bias, confounding, and incomplete data are difficult to avoid, given the dynamic nature of airway management in the field. Specifically, use of more than one airway approach was common, yet the order, detail, and duration of use was rarely adequately documented and included in analyses. Additionally, the influence of prehospital ventilation was not adequately assessed in the literature, so differences noted in outcomes after various airway management strategies may actually be related to the ventilation provided and not the airway method. Variations within types of airways based on differences in devices and training made generalizations difficult. Finally, there was a lack of evidence focusing on pediatric prehospital airway management.

\section{Conclusion and Implications}

Overall, this review found no strongly supported differences in primary outcomes, with most of the results being "no difference" across the three common methods of airway management in prehospital care. Whereas this may be due in part to study limitations, it also may reflect the reality that no one airway approach is consistently more effective across different patient needs and the widely variable prehospital environment. Attempting to derive algorithmic protocols that identify single approach recommendations based solely on effectiveness may not be possible or desirable given this heterogeneity. Future research should focus on rigorous studies, particularly RCTs, given the multiple possible sources of bias and confounding in studies of prehospital airway management that are difficult to address in observational research designs. This research should focus on patient subgroups and factors where there are inconsistencies in the currently available evidence. 


\section{Introduction}

\section{Background}

Airway management is one of the most important aspects of prehospital care. It is critical to patient survival and it affects the potential for recovery from emergent illness or injury. Effective airway management ensures airway patency to allow for oxygenation and ventilation and may protect against aspiration depending on the management approach. The primary objective in the prehospital setting is to ensure adequate oxygenation and ventilation until the transfer of patient care to an emergency department (ED) or hospital.

Historically, endotracheal intubation (ETI) has been considered the gold standard for airway management. However, while this may be true in a controlled environment, prehospital setting success rates vary, and high rates of complications attributed to a range of factors have been reported. ${ }^{1-5}$ In addition, different airways require management that involves varying levels of invasiveness or complexity, as well as distinct technologies and expertise. The simplest approaches are part of general first aid, while the most complex involve the use of drugs and surgical techniques. Basic airway management includes the use of manual maneuvers (e.g., jaw thrust or chin lift) and simple airway adjuncts (e.g., oropharyngeal airway or nasopharyngeal airway), which are devices inserted orally or nasally to facilitate airway patency. Ventilation is often achieved using a bag valve mask (BVM). In addition to ETI, other advanced airway management techniques include placement of supraglottic airway (SGA) devices, pharmacologically facilitated intubation (rapid sequence intubation [RSI], delayed sequence intubation [DSI], or sedation-facilitated intubation without paralytics), and percutaneous or surgical techniques. (Note: We use the term "supraglottic airway" in this report to describe various "extraglottic airway" methods. While "extraglottic airway" may be more technically correct, we use the term "supraglottic airway" - due to its more common use in the literature - to classify advanced airway devices that are placed outside of the trachea to facilitate oxygenation and ventilation.)

The choice of technique and the potential for success depend on the setting, severity of the patient's condition, training and skill level of emergency medical services (EMS) personnel, and available equipment. Field personnel without SGA or ETI training can perform basic maneuvers.

The challenge in prehospital airway management is to determine the appropriate approach given patient needs, and the skills and equipment available. Addressing this challenge includes considering a wide range of issues such as: (1) correct identification of patients appropriate for prehospital airway management, (2) appropriate use of advanced techniques, (3) what provider level should be certified to perform different prehospital airway interventions, (4) comparison of the benefit and harms across different airway management approaches (basic and advanced), (5) types of devices to use, (6) the setting for the airway intervention (e.g., on scene or during transport), (7) first-pass and overall success rates for advanced airway insertion, and (8) influence of patient characteristics on success rates (e.g., cardiac vs. noncardiac, trauma vs. nontrauma, traumatic brain injury vs. no brain injury, age, and comorbidities). Thus, a core decisional dilemma in prehospital care is to match the airway management approach with the needs of the patient, the resources available, and EMS personnel training and experience, to select the strategies most likely to produce the best patient outcomes.

In addition, prehospital airway management is related to several practice and policy challenges that influence the quality of prehospital care. One policy challenge is defining the skill levels for different personnel classifications and estimating how many EMS providers at 
each level are required to meet the needs of each community. Another is that barriers differ across rural and urban communities, with prehospital care playing a particularly vital role in areas with long transport-to-hospital distances $/$ time $^{6}$ and underserved areas. Furthermore, direct linkages among prehospital care and inpatient, outpatient, and emergency care have been established and strengthened by technology (e.g., telehealth) and organizational changes. These are transforming prehospital care and contributing to higher quality care as EMS becomes integrated into learning healthcare systems and health information exchange systems.

A key challenge is determining the comparative effectiveness, and balancing potential benefits and harms, of the use of different airway approaches for individual patients, given the considerations described above. This is made more difficult by the lack of a definitive gold standard in prehospital care and the wide range of possible prehospital care scenarios. ${ }^{1-5}$

Guideline developers and EMS system leaders wish to develop recommendations based on research in an environment of expanding options for prehospital airway management. Evidencebased guidelines are needed to establish a standardized approach to airway management in the prehospital setting, and national and local efforts are currently underway.

EMS agencies are part of larger healthcare systems and are essential components of the healthcare safety net for many communities. Medical direction is now required for all levels of prehospital personnel, and the most seriously ill or injured patients seen in the ED often arrive through EMS. Expanded EMS system capacities, including the availability of data collection and information integration, have made possible research examining the relationships between prehospital care and patient outcomes. As a result, there is now a body of literature that may provide evidence about the association of airway management approaches with outcomes across different types of patients and environments.

\section{Purpose and Scope of the Systematic Review}

The purpose of this systematic review was to identify and synthesize the evidence available to support the development of evidence-based recommendations and guidelines for prehospital airway management. The sponsoring funder in this effort was the National Highway Traffic Safety Administration (NHTSA), Office of Emergency Medical Services, who will utilize the review as a foundation for developing guidelines.

Specifically, this review focused on comparing the benefits and harms across three different airway management approaches: BVM, SGA, and ETI. Given the possible variations in the prehospital setting, this review considered how the benefits and harms may differ across the following factors: (1) specific techniques and devices used for each airway management approach, (2) the characteristics of the EMS personnel (e.g., training, certification, and expertise), and (3) patient characteristics (e.g., demographics, type and severity of illness or injury, and the patient location/environment).

Factors that influenced the scope of this systematic review include:

- The safety, efficacy, and risks from pharmacologically facilitated prehospital intubation when utilized;

- The likelihood that multiple attempts or delays increase the probability of poor outcomes;

- Challenges in triage and decision making outside the hospital;

- The initial and ongoing training as well as maintenance of skill needed for the different airway management techniques;

- The availability of new advanced supraglottic devices which may be easier to utilize and provide effective oxygenation and ventilation in the prehospital setting; 
- Uncertainty surrounding the role of recent initiatives such as video laryngoscopy and use of the gum elastic bougie.

Research exists on these topics, but in most cases, individual studies are not sufficient to inform policy as they are conducted in single populations or environments, may ask narrow questions, or are unable to reach definitive conclusions. In this report, we aggregated the individual studies both quantitatively and qualitatively to provide a synthesis of the evidence on the comparative benefits and harms from the use of BVM, SGA, and ETI, modified by techniques or devices used, provider characteristics, and patient characteristics. 


\section{Methods}

\section{Review Approach}

This systematic review followed the methods suggested in the Agency for Healthcare Research and Quality (AHRQ) Methods Guide for Effectiveness and Comparative Effectiveness Reviews ${ }^{7}$ (hereafter "AHRQ Methods Guide"). All methods were determined a priori, and a protocol was published on the AHRQ website and submitted to PROSPERO, a systematic reviews registry (registration no. CRD42020170201). Below is a summary of the specific methods used in this review. A more detailed description of methods, including literature search strategies, is provided in Appendix A.

\section{Key Questions}

Key Questions were posted for public comment November 22 through December 20, 2019. Comments received emphasized the value of stratifying results as much as possible by modifiers such as airway types, patient characteristics, and provider level of training and experience. The need for precision in definitions was emphasized and comments suggested review of new technologies. Concern was expressed about the ability of the literature to reflect and report on unrecognized failures to provide adequate airway management. Public comments were considered to inform the review process. Revisions were made for clarity of definitions and inclusion of new technology was confirmed.

\section{Key Question 1}

a. What are the comparative benefits and harms of bag valve mask versus supraglottic airway for patients requiring prehospital ventilatory support or airway protection?

b. Are the comparative benefits and harms modified by:

i. Techniques or devices used?

ii. Characteristics of emergency medical services personnel (including training, proficiency, experience, certification, licensure level, and/or scope of practice level)?

iii. Patient characteristics?

\section{Key Question 2}

a. What are the comparative benefits and harms of bag valve mask versus endotracheal intubation for patients requiring prehospital ventilatory support or airway protection?

b. Are the comparative benefits and harms modified by:

i. Techniques or devices used?

ii. Characteristics of emergency medical services personnel (including training, proficiency, experience, certification, licensure level, and/or scope of practice level)?

iii. Patient characteristics? 


\section{Key Question 3}

a. What are the comparative benefits and harms of supraglottic airway versus endotracheal intubation for patients requiring prehospital ventilatory support or airway protection?

b. Are the comparative benefits and harms modified by:

i. Techniques or devices used?

ii. Characteristics of emergency medical services personnel (including training, proficiency, experience, certification, licensure level, and/or scope of practice level)?

iii. Patient characteristics?

\section{Key Question 4}

What are the comparative benefits and harms of the following variations of any one of the three included airway interventions (bag valve mask, supraglottic airways, or endotracheal intubation) for patients requiring prehospital ventilatory support or airway protection:

i. Techniques or devices used?

ii. Characteristics of emergency medical services personnel (including training, proficiency, experience, certification, licensure level, and/or scope of practice level)?

iii. Patient characteristics? 


\section{Analytic Framework}

Figure 1 presents the analytic framework.

Figure 1. Analytic framework

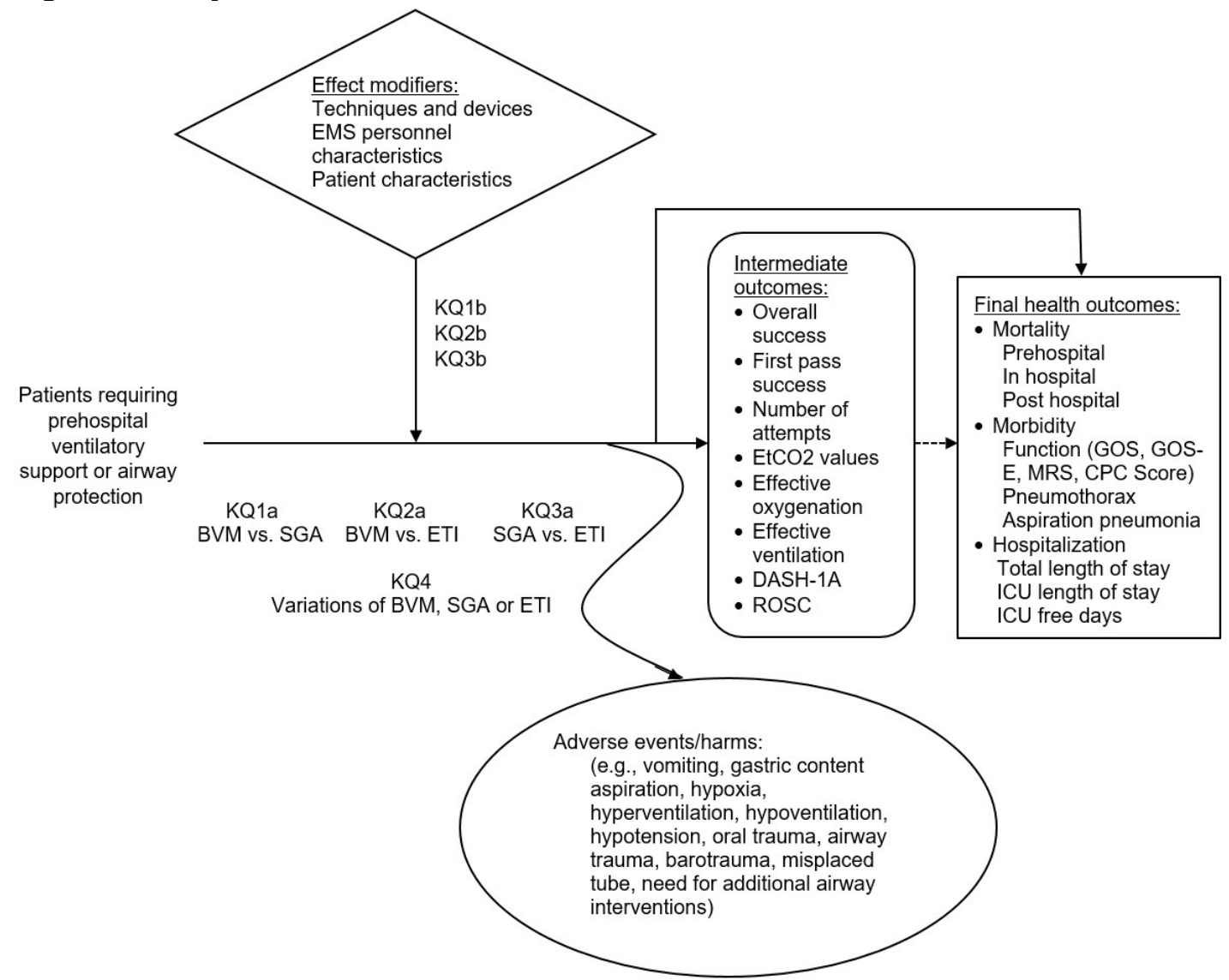

BVM = bag valve mask; CPC Score = Cerebral Performance Category Score; DASH-1A = Definitive Airway Sans Hypoxia on First Attempt; EMS = emergency medical services; ETI = endotracheal intubation; GOS = Glasgow Outcome Scale; GOS-E = Glasgow Outcome Scale Extended: Hypoxia/Hypotension on First Attempt; ICU = intensive care unit; KQ = Key Question; MRS $=$ modified Rankin Scale; ROSC $=$ return of spontaneous circulation; SGA = supraglottic airway

\section{Study Selection}

Criteria used to triage abstracts and review full texts of research articles for inclusion and exclusion were pre-established, in accordance with the AHRQ Methods Guide, ${ }^{7}$ and were developed based on the Key Questions and population, intervention, comparator, outcome, setting, study design (PICOS) specified for this project (populations, interventions, comparators, outcomes, setting; see Appendix A). To ensure accuracy, all excluded abstracts were dual reviewed to confirm exclusion. All abstracts deemed potentially appropriate for inclusion by at least one reviewer triggered retrieval of the full-text article. Each full-text article, including any articles suggested by peer reviewers or any that arose from the public posting process, was then independently reviewed for eligibility by two team members. During full-text review, all randomized controlled trials (RCTs) and comparative observational studies were retained and categorized according to which Key Questions they addressed. The literature flow appears in Appendix B. 
Authors of a paper who were on the research team did not review their own publications. Disagreements between two team members regarding study inclusion were resolved by consensus of the investigators involved.

\section{Data Extraction and Risk of Bias Assessment}

After studies were selected for inclusion, data were abstracted including study design, year, setting, country, sample size, eligibility criteria, population and clinical characteristics, intervention characteristics, and results relevant to each Key Question, as outlined in the PICOS table (Appendix A). Data from included studies (Appendix C) were abstracted into an interactive database to facilitate meta-analyses. All abstracted data were verified for accuracy and completeness by a second team member. A record of studies excluded at the full-text level with reasons for exclusion is provided in Appendix D.

Predefined criteria were used to assess the quality of included studies. Study design-specific criteria were used, as recommended in the chapter, "Assessing the Risk of Bias of Individual Studies When Comparing Medical Interventions" in the AHRQ Methods Guide. ${ }^{7}$ Randomized controlled trials were evaluated using Cochrane risk of bias criteria, ${ }^{8}$ and observational studies were evaluated using criteria developed by the U.S. Preventive Services Task Force. ${ }^{9}$

For full data extraction (Appendix E and Appendix F) and risk of bias assessment (Appendix G), please see the Methods Appendix A.

\section{Data Synthesis and Analysis}

We constructed evidence tables including study characteristics, results, and quality ratings for all included studies (Appendix E and Appendix F), along with summary tables that highlight the main findings provided in the Results section of this report. Results were organized by Key Question and stratified by major subgroups.

\section{Definitions and Outcome Measures}

Studies in which greater than 85 percent of the participants were cardiac arrest patients were categorized as cardiac arrest at the study level. Studies or subgroups were categorized as pediatric based on each study's age-based definition. Studies in which less than 10 percent of patients were pediatric were categorized as adult at the study level. Studies for which age distribution was not distinguished were categorized as "mixed-age" at the study level.

Results for all outcomes specified in the PICOS were abstracted and analyzed, either quantitatively or qualitatively. We prioritized direct patient centered outcomes for meta-analyses. These included survival to hospital discharge or 30 days, neurological function at hospital discharge or 30 days, return of spontaneous circulation (ROSC), and successful airway placement on the first attempt (first-pass success) and overall (overall success).

\section{Meta-Analyses}

Meta-analyses (Appendix $\mathrm{H}$ and Appendix I), using profile-likelihood random effects model ${ }^{10}$ were conducted to summarize data and obtain more precise estimates where there are at least two studies reporting outcomes that were homogeneous enough to provide a meaningful combined estimate. To determine whether meta-analyses were appropriate, we considered the quality of individual studies, the heterogeneity across several variables including patient characteristics, interventions, and outcomes, as well as the completeness of the same reported 
outcomes. All meta-analyzable outcomes were binary and risk ratio (RR) was the effect measure. Adjusted RRs or odds ratios (OR) were used in the meta-analysis if reported (an adjusted OR was first converted to an adjusted RR). ${ }^{11}$ Otherwise, the RR was calculated from the reported raw numbers. Statistical heterogeneity was assessed using the $\chi^{2}$ test, and the magnitude of heterogeneity using the $I^{2}$ statistic. $^{12}$

The Key Questions were designed to assess the comparative effectiveness and harms by airway intervention, emergency medical services personnel, and patient characteristics. Therefore, stratified analyses were conducted based on study design (i.e., RCTs or observational studies), emergency type (e.g. cardiac arrest, trauma), and population age (adult, pediatric, or mixed-age). Controlled clinical trials were grouped with either RCTs or observational studies in meta-analyses based on the characteristics of the study. If a study provided data for more than one definition of ROSC, we used in order of preference: sustained ROSC, any ROSC, prehospital ROSC. For neurological function, we did not pool across different assessment measures. Studies with mixed-age populations were grouped with the adult studies for stratification in the primary analyses. In primary analyses, we used data from the intent-to-treat analysis for RCTs, and if reported, propensity score matched results for observational studies. Sensitivity analyses were conducted by using other reported data (e.g., data from per-protocol, or as treated analysis for RCTs, unadjusted results), or by excluding studies with outlying results, those rated as high risk of bias, and studies in mixed-age populations, as separate analyses.

All analyses were performed by using STATA ${ }^{\circledR} 16.1$ (StataCorp, College Station, TX), and all results were reported with 95 percent confidence intervals (95\% CIs).

\section{Qualitative Synthesis}

Where pooling studies was not appropriate, qualitative syntheses, which include summary tables, tabulations of important study features, and narratives, were created and are presented by Key Questions and outcomes (see Results chapter and Appendix F).

\section{Grading the Strength of the Body of Evidence}

Regardless of whether evidence was synthesized quantitatively or qualitatively, the strength of evidence (Appendix J) for each Key Question/body of evidence was initially assessed by one researcher for each clinical outcome (see PICOS, Appendix Table A-1) by using the approach described in the AHRQ Methods Guide. ${ }^{7}$ To ensure consistency and validity of the evaluation, the strength of evidence was reviewed by one or more additional investigators prior to assigning a final grade, based on the following factors:

- Risk of bias across included studies (low, moderate, or high level of risk of bias)

- Consistency of results (consistent, inconsistent, or unknown/not applicable)

- Directness (direct or indirect)

- Precision of effect estimates (precise or imprecise)

- Reporting bias (suspected or undetected)

The strength of evidence (SOE) was determined for each outcome by each airway comparison (see Appendix J). RCTs and observational studies were not mixed in the pooled estimates and are reported separately by study design. However, the SOE assessment is for the entire body of evidence, based on the totality of evidence across study designs. In making the SOE determination and specifying what can be concluded from the evidence, RCTs with low or 
moderate risk of bias were prioritized over observational studies. In addition, if findings from observational studies conflicted with those of RCTs, the conclusion and final SOE were based on findings from the RCTs.

For description of overall grades, please see Methods (Appendix A). 


\section{Results}

\section{Introduction}

Our literature search produced 9,284 abstracts of potentially relevant articles. We reviewed 772 full-text publications. Of those, 99 studies from 101 publications involving 630,397 patients were included for this review (see Appendix B, Literature Flow).

In this section we presented the results of our quantitative and qualitative analyses of the studies included for the review that addressed one or more of the four Key Questions. We began with a summary of the overall findings across Key Questions, and then provided specific results for each individual Key Question. The list of included studies can be found in Appendix C. Table 1 presents characteristics of the included studies. Table 2 shows the number of studies by study design.

For quantitative analysis, we identified outcomes with studies that could be combined in meta-analyses (see Appendix H and I). These were survival in-hospital or at 1-month post incident for all Key Questions; neurological function at discharge or 1-month post incident, and return of spontaneous circulation (ROSC) for Key Questions 1-3; and successful advanced airway insertion for Key Questions 3 and 4 only (Tables 3-5). Forest plots for the primary analyses are presented in Appendix H. In the sections below that address individual Key Questions, we provided tables with the number of studies and number of patients included in the studies, and the pooled risk ratio with confidence interval and $\mathrm{I}^{2}$ for randomized controlled trials (RCTs) and observational studies separately. The overall strength of evidence (SOE) was provided for age and emergency type subgroup.

Details on the determinations that contributed to the SOE are in Appendix J. We conducted sensitivity analyses for all outcomes and discussed relevant findings in the individual sections below (forest plots for sensitivity analyses are included in Appendix I).

For qualitative analysis, we summarized studies that could not be included in meta-analyses and present the findings in the sections below that address individual Key Questions.

Table 1. Characteristics of included studies

\begin{tabular}{|c|c|c|c|c|c|c|}
\hline Category & Characteristics & $\begin{array}{l}\text { Overall } \\
\mathbf{N}(\%) \\
N=99^{a}\end{array}$ & $\begin{array}{l}\text { KQ1 } \\
N=22^{a}\end{array}$ & $\begin{array}{l}\text { KQ2 } \\
N=22^{a}\end{array}$ & $\begin{array}{l}\text { KQ3 } \\
N=41^{a}\end{array}$ & $\begin{array}{l}\text { KQ4 } \\
N=51\end{array}$ \\
\hline \multirow{3}{*}{$\begin{array}{l}\text { Year of } \\
\text { Publication }\end{array}$} & $1990-2000$ & $9(9.1 \%)$ & $1(4.5 \%)$ & $2(9.1 \%)$ & $3(7.3 \%)$ & $5(9.8 \%)$ \\
\hline & $2001-2010$ & $22(22.2 \%)$ & $5(22.7 \%)$ & $7(31.8 \%)$ & $9(22.0 \%)$ & $10(19.6 \%)$ \\
\hline & $2011-2020$ & $68(68.7 \%)$ & $16(72.7 \%)$ & $13(59.1 \%)$ & $29(70.7 \%)$ & $36(70.6 \%)$ \\
\hline \multirow{4}{*}{ Study Design } & $\mathrm{RCT}$ & $22(22.2 \%)$ & $4(18.2 \%)$ & $4(18.2 \%)$ & $5(12.2 \%)$ & $14(27.5 \%)$ \\
\hline & $\begin{array}{l}\text { Prospective } \\
\text { observational }\end{array}$ & $20(20.2 \%)$ & $5(22.7 \%)$ & $3(13.6 \%)$ & $10(24.4 \%)$ & $9(17.5 \%)$ \\
\hline & $\begin{array}{l}\text { Retrospective } \\
\text { observational }\end{array}$ & $50(50.5 \%)$ & $12(54.5 \%)$ & $15(68.2 \%)$ & $25(61.0 \%)$ & $23(45.1 \%)$ \\
\hline & Before/after & $7(7.1 \%)$ & $1(4.5 \%)$ & 0 & $1(2.4 \%)$ & $5(9.8 \%)$ \\
\hline \multirow{6}{*}{$\begin{array}{l}\text { Geographic } \\
\text { Location }\end{array}$} & $\begin{array}{l}\text { United } \\
\text { States/Canada }\end{array}$ & $48(48.5 \%)$ & $8(36.4 \%)$ & $10(45.5 \%)$ & $21(51.2 \%)$ & $26(51.0 \%)$ \\
\hline & Europe & $26(26.3 \%)$ & $4(18.2 \%)$ & $2(9.1 \%)$ & $8(19.5 \%)$ & $14(27.5 \%)$ \\
\hline & Asia & $17(17.2 \%)$ & $10(45.5 \%)$ & $9(40.9 \%)$ & $11(26.8 \%)$ & $4(7.8 \%)$ \\
\hline & Africa & $1(1.0 \%)$ & $0(0.0 \%)$ & $0(0.0 \%)$ & $0(0.0 \%)$ & $1(2.0 \%)$ \\
\hline & Australia & $6(6.1 \%)$ & $0(0.0 \%)$ & $1(4.5 \%)$ & $1(2.4 \%)$ & $5(9.8 \%)$ \\
\hline & Multiple countries & $1(1.0 \%)$ & $0(0.0 \%)$ & $0(0.0 \%)$ & $0(0.0 \%)$ & $1(2.0 \%)$ \\
\hline \multirow{3}{*}{$\begin{array}{l}\text { Prehospital } \\
\text { Setting }\end{array}$} & Urban & $46(46.5 \%)$ & $9(40.9 \%)$ & $13(59.1 \%)$ & $20(48.8 \%)$ & $23(45.1 \%)$ \\
\hline & Rural & $3(3.0 \%)$ & $1(4.5 \%)$ & $0(0.0 \%)$ & $1(2.4 \%)$ & $1(2.0 \%)$ \\
\hline & Mixed & $32(32.3 \%)$ & $7(31.8 \%)$ & $6(27.3 \%)$ & $16(39.0 \%)$ & $14(29.4 \%)$ \\
\hline
\end{tabular}




\begin{tabular}{|c|c|c|c|c|c|c|}
\hline Category & Characteristics & $\begin{array}{l}\text { Overall } \\
N(\%) \\
N=99^{a}\end{array}$ & $\begin{array}{l}\text { KQ1 } \\
\mathrm{N}=22^{\mathrm{a}}\end{array}$ & $\begin{array}{l}\text { KQ2 } \\
\mathrm{N}=22^{\mathrm{a}}\end{array}$ & $\begin{array}{l}\text { KQ3 } \\
\mathrm{N}=41^{\mathrm{a}}\end{array}$ & $\begin{array}{l}\text { KQ4 } \\
\mathrm{N}=51\end{array}$ \\
\hline & Not reported & $18(18.2 \%)$ & $5(22.7 \%)$ & $3(13.6 \%)$ & $4(9.8 \%)$ & $12(23.5 \%)$ \\
\hline \multirow{3}{*}{$\begin{array}{l}\text { Number of } \\
\text { Agencies/ } \\
\text { Institutions }\end{array}$} & Single & $44(44.4 \%)$ & $6(27.3 \%)$ & $7(31.8 \%)$ & $15(36.6 \%)$ & $28(54.9 \%)$ \\
\hline & Multiple & $54(54.5 \%)$ & $16(72.7 \%)$ & $15(68.2 \%)$ & $26(63.4 \%)$ & $22(43.1 \%)$ \\
\hline & Not reported & $1(1.0 \%)$ & $0(0.0 \%)$ & $0(0.0 \%)$ & $0(0.0 \%)$ & $1(2.0 \%)$ \\
\hline \multirow{5}{*}{$\begin{array}{l}\text { EMS Provider } \\
\text { Level }^{\text {b }}\end{array}$} & ETI-capable No & $7(7.1 \%)$ & $5(22.7 \%)$ & $0(0.0 \%)$ & $0(0.0 \%)$ & $3(5.9 \%)$ \\
\hline & ETI-capable Yes & $40(40.4 \%)$ & $7(31.8 \%)$ & $11(50.0 \%)$ & $15(36.6 \%)$ & $24(47.1 \%)$ \\
\hline & Advanced & $15(15.2 \%)$ & $1(4.5 \%)$ & $1(4.5 \%)$ & $1(2.4 \%)$ & $12(23.5 \%)$ \\
\hline & Mixed & $36(36.4 \%)$ & $9(40.9 \%)$ & $10(45.5 \%)$ & $25(61.0 \%)$ & $11(21.6 \%)$ \\
\hline & Not reported & $1(1.0 \%)$ & $0(0.0 \%)$ & $0(0.0 \%)$ & $0(0.0 \%)$ & $1(2.0 \%)$ \\
\hline \multirow{4}{*}{$\begin{array}{l}\text { Mode of } \\
\text { Transport }\end{array}$} & Ground & $61(61.6 \%)$ & $14(63.6 \%)$ & $13(59.1 \%)$ & $28(68.3 \%)$ & $29(56.9 \%)$ \\
\hline & Air & $10(10.1 \%)$ & $0(0.0 \%)$ & $0(0.0 \%)$ & $0(0.0 \%)$ & $10(19.6 \%)$ \\
\hline & Mixed & $17(17.2 \%)$ & $3(13.6 \%)$ & $3(13.6 \%)$ & $7(17.1 \%)$ & $8(15.7 \%)$ \\
\hline & Not reported & $11(11.1 \%)$ & $5(22.7 \%)$ & $6(27.3 \%)$ & $6(14.6 \%)$ & $4(7.8 \%)$ \\
\hline \multirow{4}{*}{ Age Group } & Pediatric & $7(7.1 \%)$ & $2(9.1 \%)$ & $4(18.2 \%)$ & $3(7.3 \%)$ & $3(5.9 \%)$ \\
\hline & Adult & $81(81.8 \%)$ & $19(86.4 \%)$ & $16(72.7 \%)$ & $36(87.8 \%)$ & $40(78.4 \%)$ \\
\hline & Mixed & $11(11.1 \%)$ & $1(4.5 \%)$ & $2(9.1 \%)$ & $2(4.9 \%)$ & $8(15.7 \%)$ \\
\hline & Not reported & $0(0.0 \%)$ & $0(0.0 \%)$ & $0(0.0 \%)$ & $0(0.0 \%)$ & $0(0.0 \%)$ \\
\hline \multirow{5}{*}{ Emergency Type } & Trauma & $15(15.2 \%)$ & $1(4.5 \%)$ & $4(18.2 \%)$ & $2(4.9 \%)$ & $9(17.6 \%)$ \\
\hline & Cardiac arrest & $49(49.5 \%)$ & $21(95.5 \%)$ & $17(77.3 \%)$ & $31(75.6 \%)$ & $13(25.5 \%)$ \\
\hline & Medical & $3(3.0 \%)$ & $0(0.0 \%)$ & $0(0.0 \%)$ & $2(4.9 \%)$ & $1(2.0 \%)$ \\
\hline & Mixed & $30(30.3 \%)$ & $0(0.0 \%)$ & $1(4.5 \%)$ & $6(14.6 \%)$ & $26(51.0 \%)$ \\
\hline & Not reported & $2(2.0 \%)$ & $0(0.0 \%)$ & $0(0.0 \%)$ & $0(0.0 \%)$ & $2(3.9 \%)$ \\
\hline
\end{tabular}

EMS = emergency medical services; ETI = endotracheal intubation; KQ = Key Question; RCT = randomized controlled trial

${ }^{a}$ Evans, 2016 counted as two studies

${ }^{\mathrm{b}}$ EMS Provider Level Categorization: Two topic experts reviewed all included studies and categorized EMS Provider Levels as ETI-capable No; ETI-capable Yes; Advanced (physicians, nurses, physician assistants) or Mixed levels (the EMS team included two or more of these 3 provider levels).

Table 2. Number of studies by Key Question and study design

\begin{tabular}{|l|l|l|l|}
\hline Key Question & $\begin{array}{l}\text { Randomized Controlled } \\
\text { Trials }\end{array}$ & Observational Studies & Total \\
\hline Key Question 1 & 4 & 18 & 22 \\
\hline Key Question 2 & 4 & 18 & 22 \\
\hline Key Question 3 & 5 & 36 & 41 \\
\hline Key Question 4 & 14 & 37 & 51 \\
\hline
\end{tabular}

\section{Summary of Overall Results}

The overall results are summarized in the bullet points and Table 3. Detailed results are presented in the individual Key Question sections.

- Survival measured in-hospital or at 1-month post incident:

- No difference in outcomes across all three comparisons in adult/mixed-age patients with cardiac arrest and pediatric patients with cardiac arrest.

o No difference when bag valve mask (BVM) was compared with endotracheal intubation (ETI) in adult trauma patients.

- Neurological function measured by the Cerebral Performance Category (CPC), Pediatric CPC, or modified Rankin Scale (mRS) in-hospital or at 1-month post incident:

○ When BVM was compared with supraglottic airway (SGA), outcomes favored BVM in adult patients with cardiac arrest.

- When SGA was compared with ETI, outcomes measured by the CPC favored ETI in adult patients with cardiac arrest; there was no difference in outcomes measured by the mRS in this group. 
$\circ$ When BVM was compared with ETI, there was no difference in outcomes in adult patients with cardiac arrest.

- When ETI was compared with BVM or SGA, there was no difference in outcomes in pediatric patients with cardiac arrest.

- $\quad$ ROSC (prehospital, sustained, or overall - cardiac arrest patients only):

$\circ$ When BVM was compared with SGA or ETI, there was no difference in outcomes in adult cardiac arrest patients.

- When SGA was compared with ETI, outcomes favored SGA in adult patients with cardiac arrest.

- When ETI was compared with BVM or SGA, there was no difference in outcomes in pediatric patients with cardiac arrest.

- Successful advanced airway insertion when SGA is compared with ETI:

- First-pass success favors SGA in adults with cardiac arrest and with mixed emergency types, and in pediatric patients with cardiac arrest; no difference in adults with medical emergencies.

- No difference in overall success in adults with cardiac arrest, medical emergencies, or mixed emergency types.

Table 3. Overview of conclusions: comparisons by emergency types and age groups

\begin{tabular}{|c|c|c|c|c|}
\hline Outcome & Emergency Type and Age & $\begin{array}{l}\text { KQ1: } \\
\text { BVM vs. SGA }\end{array}$ & $\begin{array}{l}\text { KQ2: } \\
\text { BVM vs. ETI }\end{array}$ & $\begin{array}{l}\text { KQ3: } \\
\text { SGA vs. ETI }\end{array}$ \\
\hline \multirow{4}{*}{ Survival } & $\begin{array}{l}\text { Cardiac arrest: } \\
\text { Adults/Mixed }\end{array}$ & No difference & No difference & No difference \\
\hline & Cardiac arrest: Pediatrics & No difference ${ }^{a}$ & No difference & No difference ${ }^{a}$ \\
\hline & Trauma: Adults & No conclusion ${ }^{a}$ & No difference & No conclusion ${ }^{a}$ \\
\hline & Trauma: Pediatrics & No evidence & No conclusion a & No evidence \\
\hline \multirow{4}{*}{$\begin{array}{l}\text { Neurological } \\
\text { Function }\end{array}$} & Cardiac arrest: Adults & $\begin{array}{l}\text { mRS: No evidence } \\
\text { CPC: Favors BVM }\end{array}$ & $\begin{array}{l}\text { mRS: No evidence } \\
\text { CPC: No } \\
\text { difference }\end{array}$ & $\begin{array}{l}\text { mRS: No difference } \\
\text { CPC: Favors ETIa }\end{array}$ \\
\hline & Cardiac arrest: Pediatrics & No conclusion ${ }^{a}$ & No difference & No difference ${ }^{a}$ \\
\hline & Trauma: Adults & No evidence & No evidence & No evidence \\
\hline & Trauma: Pediatrics & No evidence & No evidence & No evidence \\
\hline \multirow{2}{*}{ ROSC $^{b}$} & Cardiac arrest: Adults & No difference & No difference & Favors SGA \\
\hline & Cardiac arrest: Pediatrics & No conclusion ${ }^{a}$ & No difference ${ }^{a}$ & No difference ${ }^{a}$ \\
\hline \multirow{8}{*}{$\begin{array}{l}\text { First-Pass } \\
\text { Success }^{c}\end{array}$} & Cardiac arrest: Adults & NA & NA & Favors SGA \\
\hline & Cardiac arrest: Pediatrics & NA & $\mathrm{NA}$ & Favors SGA ${ }^{a}$ \\
\hline & Trauma: Adults & NA & NA & No conclusion ${ }^{\mathrm{a}}$ \\
\hline & Trauma: Pediatrics & NA & $\mathrm{NA}$ & No conclusion a \\
\hline & Medical: Adults & NA & NA & No difference \\
\hline & Medical: Pediatrics & NA & $\mathrm{NA}$ & No conclusion a \\
\hline & Mixed: Adults & NA & $\mathrm{NA}$ & Favors SGA ${ }^{a}$ \\
\hline & Mixed: Pediatrics & NA & NA & No evidence \\
\hline \multirow{8}{*}{$\begin{array}{l}\text { Overall } \\
\text { Success }^{c}\end{array}$} & Cardiac arrest: Adults & NA & NA & No difference \\
\hline & Cardiac arrest: Pediatrics & NA & NA & No evidence \\
\hline & Trauma: Adults & NA & NA & No conclusion ${ }^{a}$ \\
\hline & Trauma: Pediatrics & NA & NA & No evidence \\
\hline & Medical: Adults & NA & $\mathrm{NA}$ & No difference \\
\hline & Medical: Pediatrics & NA & NA & No evidence \\
\hline & Mixed: Adults & NA & NA & No difference ${ }^{a}$ \\
\hline & Mixed: Pediatrics & NA & $\mathrm{NA}$ & No evidence \\
\hline
\end{tabular}




\begin{tabular}{|l|l|l|l|l|}
\hline Outcome & Emergency Type and Age & $\begin{array}{l}\text { KQ1: } \\
\text { BVM vs. SGA }\end{array}$ & $\begin{array}{l}\text { KQ2: } \\
\text { BVM vs. ETI }\end{array}$ & $\begin{array}{l}\text { KQ3: } \\
\text { SGA vs. ETI }\end{array}$ \\
\hline & & & & $\begin{array}{l}\text { No difference: } \\
\text { Aspiration, } \\
\text { oral/airway trauma, } \\
\text { regurgitation }\end{array}$ \\
& & & & $\begin{array}{l}\text { Favors SGA: } \\
\text { Marms }\end{array}$ \\
& All groups & No difference & attempts \\
& & No difference \\
& & & & $\begin{array}{l}\text { Favors ETI: } \\
\text { Inadequate } \\
\text { ventilation }\end{array}$ \\
\hline
\end{tabular}

$\mathrm{BVM}=$ bag valve mask; $\mathrm{CPC}=$ Cerebral Performance Category; ETI = endotracheal intubation; KQ = Key Question; $\mathrm{mRS}=$ modified Rankin Scale; NA = not applicable; ROSC = return of spontaneous circulation; SGA = supraglottic airway

Bold Text $=$ Moderate SOE, Standard text $=$ Low SOE, Italicized text $=$ Insufficient SOE

${ }^{a}$ Results based only on observational studies

${ }^{\mathrm{b}} \mathrm{ROSC}$ was only reported in studies of cardiac arrest

${ }^{c}$ Success was qualitatively synthesized for KQ1 and 2; results available in full report

${ }^{\mathrm{d}}$ Harms were qualitatively synthesized; meta-analysis not possible as harms are different

Table 4. Overview of conclusions: successful advanced airway insertion by emergency type and age-Key Question 3

\begin{tabular}{|l|l|l|}
\hline Outcome & Emergency Type and Age & KQ3: SGA vs. ETI \\
\hline \multirow{5}{*}{ First-Pass Success } & Cardiac arrest: Adults & Favors SGA \\
\cline { 2 - 3 } & Cardiac arrest: Pediatrics & Favors SGA \\
\cline { 2 - 3 } & Trauma: Adults & No conclusion \\
\cline { 2 - 3 } & Trauma: Pediatrics & No conclusion \\
\cline { 2 - 3 } & Medical: Adults & No difference \\
\cline { 2 - 3 } & Medical: Pediatrics & No conclusion \\
\cline { 2 - 3 } & Mixed: Adults & Favors SGA \\
\cline { 2 - 3 } & Mixed: Pediatrics & No evidence \\
\hline \multirow{5}{*}{ Overall Success } & Cardiac arrest: Adults & No difference \\
\cline { 2 - 3 } & Cardiac arrest: Pediatrics & No evidence \\
\cline { 2 - 3 } & Trauma: Adults & No conclusion \\
\cline { 2 - 3 } & Trauma: Pediatrics & No evidence \\
\cline { 2 - 3 } & Medical: Adults & No difference \\
\cline { 2 - 3 } & Medical: Pediatrics & No evidence \\
\cline { 2 - 3 } & Mixed: Adults & No difference \\
\cline { 2 - 3 } & Mixed: Pediatrics & No evidence \\
\hline
\end{tabular}

ETI = endotracheal intubation; KQ = Key Question; SGA = supraglottic airway

Bold Text $=$ Moderate SOE, Standard text $=$ Low SOE, Italicized text $=$ Insufficient SOE

${ }^{\text {a }}$ Results based only on observational studies 
Table 5. Overview of conclusions: outcomes for modifiers of endotracheal intubation-Key Question 4

\begin{tabular}{|c|c|c|c|}
\hline Outcome & Emergency Type & $\begin{array}{l}\text { Video Versus Direct } \\
\text { Laryngoscopy }\end{array}$ & $\begin{array}{l}\text { ETI With Drug-Facilitation } \\
\text { Versus Without }\end{array}$ \\
\hline Survival & No evidence & No evidence & $\begin{array}{l}\text { RSI vS. no medication } \\
\text { No difference: } \\
\text { - Trauma: Adults/mixed- } \\
\text { age }\end{array}$ \\
\hline $\begin{array}{l}\text { First- } \\
\text { Pass } \\
\text { Success }\end{array}$ & $\begin{array}{ll}\text { Favors medical } \\
\text { - } & \text { Trauma vs. medical: } \\
\text { No difference } \\
\text { - } \\
\text { Medical vs. cardiac } \\
\text { - } \\
\text { arrest: Adults/mixed-age } \\
\text { Trauma vs. cardiac } \\
\text { - } \quad \text { Norrest: Adults/mixed-age } \\
\quad \text { arrest: Adults/mixed-age }\end{array}$ & $\begin{array}{l}\text { No difference } \\
\text { - } \quad \text { Cardiac arrest: Adults } \\
\text { - } \quad \text { Trauma: Adults } \\
\text { - } \quad \text { Mixed emergencies: } \\
\quad \text { Adults/mixed-age }\end{array}$ & $\begin{array}{l}\text { RSI vs. no medication } \\
\text { Favors RSI: } \\
\text { - Mixed emergencies: } \\
\text { Adults/mixed-age } \\
\text { No difference: } \\
\text { - Trauma: Adults/mixed- } \\
\quad \text { age } \\
\text { RSI vs. sedation-facilitated } \\
\text { Favors RSI: } \\
\text { - Mixed emergencies: } \\
\quad \text { Adults/mixed-age } \\
\text { Sedation-facilitated vs. no } \\
\text { medication } \\
\text { No difference: } \\
\text { Mixed emergencies: } \\
\text { Adults/mixed-age }\end{array}$ \\
\hline $\begin{array}{l}\text { Overall } \\
\text { Success }\end{array}$ & $\begin{array}{ll}\text { No difference } \\
\text { - } & \text { Medical vs. cardiac } \\
& \text { arrest: Adults/mixed-age } \\
\text { - } & \text { Trauma vs. cardiac } \\
& \text { arrest: Adults/mixed-age } \\
\text { - } & \text { Nonarrest vs. cardiac } \\
\text { - } & \text { arrest: Adults/mixed-age } \\
& \text { Trauma vs. medical: } \\
& \text { Adults/mixed-age }\end{array}$ & $\begin{array}{l}\text { No difference } \\
\text { - } \quad \text { Cardiac arrest: Adults } \\
\text { - } \quad \text { Mixed emergencies: } \\
\quad \text { Adults/mixed-age }\end{array}$ & $\begin{array}{l}\text { RSI vS. no medication } \\
\text { Favored RSI: } \\
\text { - Trauma: Adults } \\
\text { No difference } \\
\text { - Cardiac arrest: Adults } \\
\text { - Mixed emergencies: } \\
\quad \text { Adults/mixed-age }\end{array}$ \\
\hline Harms & No evidence & $\begin{array}{l}\text { More often with video: problems } \\
\text { advancing the tube }\end{array}$ & $\begin{array}{l}\text { RSI vs. no medication } \\
\text { More harms with no medication: } \\
\text { recognized esophageal } \\
\text { intubation, unrecognized } \\
\text { mainstem intubation, } \\
\text { hypotension } \\
\frac{\text { RSI vs. sedation-facilitated }}{\text { No cases in either group for }} \\
\text { esophageal intubation or } \\
\text { aspiration } \\
\frac{\text { Sedation-facilitated vs. no }}{\text { medication }} \\
\text { No harms reported in studies }\end{array}$ \\
\hline
\end{tabular}

$\mathrm{ETI}=$ endotracheal intubation; $\mathrm{RSI}=$ rapid sequence intubation; $\mathrm{SOE}=$ strength of evidence Bold Text $=$ Moderate SOE, Standard Text $=$ Low SOE 


\section{Individual Key Question Summaries}

\section{Key Question 1: Bag Valve Mask Compared With Supraglottic Airway}

\section{Key Results}

When BVM was compared with SGA for prehospital airway management:

- Survival in-hospital or at 1-month post incident (17 studies; $\mathrm{N}=49,153)$ was not significantly different between BVM and SGA for:

- Adult/mixed-age cardiac arrest patients (SOE: Low)

- Pediatric cardiac arrest patients (SOE: Low)

- Neurological function, defined as good neurological outcomes at discharge or 1-month post incident measured by the CPC or Pediatric CPC (10 studies; N=92,235), favored BVM versus SGA for:

- Adult/mixed-age cardiac arrest patients (SOE: Low)

- ROSC, defined as any ROSC (13 studies; $\mathrm{N}=47,841$ ), was similar between BVM and SGA for:

- Adult cardiac arrest patients (SOE: Low)

- Harms (4 studies; N=696) were not significantly different between BVM and SGA (SOE: Moderate)

\section{Summary of Results}

We included 22 studies that compared patient outcomes for BVM and SGA ( $N=70,718)$ (See Appendix $\mathrm{C}$ for the list of included studies). These included 4 RCTs (in 5 publications), ${ }^{13-17} 5$ prospective cohorts (in 5 publications), ${ }^{18-22} 12$ retrospective cohorts (in 11 publications), ${ }^{23-33}$ and 1 before-after study. ${ }^{34}$ The studies included 50 to 45,685 participants, and 8 were conducted in the United States and Canada, ${ }^{14,16-18,24-27} 6$ in Japan, ${ }^{20,22,29-31,33} 3$ in Austria, ${ }^{13,19,21} 2$ each in Taiwan ${ }^{23,34}$ and South Korea, ${ }^{28,32}$ and 1 in France. ${ }^{15}$ Six of these studies were rated low risk of bias (ROB), ${ }^{19,20,23,25,26,31} 12$ moderate (in 11 publications), ${ }^{13,15,16,18,22,24,27,29,32-34}$ and one high. ${ }^{30}$ Three (in 4 publications) were rated low ROB on certain outcomes and moderate on others (Appendix G). ${ }^{14,17,21,28}$

\section{Meta-Analysis}

Twenty-two studies were pooled to obtain estimates for survival, neurological function, and ROSC, stratified by emergency type, age, and study design (Appendix Figures H-1 to H-3). ${ }^{13-}$ 21,23-34 The results for each outcome by emergency type and age groups are reported in Tables 6 to 8 .

\section{Survival}

There was no difference in survival comparing BVM and SGA in observational studies enrolling pediatric patients with cardiac arrest (SOE: Low) (Table 6). For adult trauma patients, SOE was insufficient, as there was only one eligible study. In studies enrolling adults with cardiac arrest, results were mixed, with observational studies favoring BVM and RCTs showing no difference. When the data were analyzed using unadjusted results, or when studies of mixedage or those rated high ROB were excluded, results for observational studies with adult cardiac 
arrest patients showed no difference between BVM and SGA (Appendix Figures I-1 to I-4). Overall, results for adults with cardiac arrest suggested no difference between BVM and SGA on in-hospital or 30-day survival (SOE: Low).

Table 6. SGA versus BVM: survival by emergency type and age group (BVM referent)

\begin{tabular}{|c|c|c|c|c|c|}
\hline $\begin{array}{l}\text { Emergency } \\
\text { Type }\end{array}$ & Age Group & $\begin{array}{l}\text { Conclusion } \\
\text { (SOE) }\end{array}$ & $\begin{array}{l}\text { Study Number } \\
\text { and Design }\end{array}$ & $\begin{array}{l}\text { Number of } \\
\text { Patients }\end{array}$ & $\begin{array}{l}\text { Summary of Results } \\
\text { RR }(95 \% \mathrm{Cl}), \mathrm{I}^{2}\end{array}$ \\
\hline \multirow{4}{*}{$\begin{array}{l}\text { Cardiac } \\
\text { Arrest }\end{array}$} & \multirow[b]{2}{*}{ Adults/Mixed } & \multirow{2}{*}{$\begin{array}{l}\text { No difference } \\
\text { (Low) }\end{array}$} & $3 \mathrm{RCT}^{14-17}$ & 2,046 & 0.31 (0.15 to 2.98$), 0 \%$ \\
\hline & & & $\begin{array}{l}11 \mathrm{OBS}^{19-} \\
25,28,30,32\end{array}$ & 45,980 & 0.65 (0.41 to 0.96$), 69.8 \%$ \\
\hline & \multirow{2}{*}{ Pediatrics } & \multirow{2}{*}{$\begin{array}{l}\text { No difference } \\
\text { (Low) }\end{array}$} & No RCT & - & - \\
\hline & & & $2 \mathrm{OBS}^{18,26}$ & 1078 & $0.52(0.17$ to 1.98$), 0 \%$ \\
\hline \multirow{3}{*}{ Trauma } & \multirow{2}{*}{ Adults } & \multirow{2}{*}{$\begin{array}{l}\text { No conclusion } \\
\text { (Insufficient) }\end{array}$} & No RCT & - & - \\
\hline & & & $1 \mathrm{OBS}^{27}$ & 50 & $0.76(0.20$ to 2.80$), \mathrm{NA}$ \\
\hline & Pediatrics & - & - & - & - \\
\hline
\end{tabular}

$\mathrm{BVM}=$ bag valve mask; $\mathrm{CI}=$ confidence interval; $\mathrm{I}^{2}=$ statistical test of heterogeneity; $\mathrm{NA}=$ not applicable; OBS $=$ observational study; $\mathrm{RCT}$ = randomized controlled trial; $\mathrm{RR}=$ risk ratio; $\mathrm{SGA}=$ supraglottic airway; $\mathrm{SOE}=$ strength of evidence

\section{Neurological Function}

Studies of neurological function at discharge or 1-month post incident were limited to patients with cardiac arrest (Table 7). BVM was associated with good neurological outcome ratings versus SGA in one RCT (2 publications) and eight observational studies enrolling adults (SOE: Low). For pediatric patients, SOE was insufficient, as there was only one eligible observational study (Table 7). Sensitivity analyses of observational studies of adults were conducted with unadjusted results and excluding studies rated high risk of bias. In these, the pooled effect for observational studies changed to no difference between BVM and SGA (Appendix Figures I-5 to I-6). No changes in effect were detected in sensitivity analyses for the RCT or pediatric studies.

Table 7. SGA versus BVM: neurological function by emergency type and age group (BVM referent)

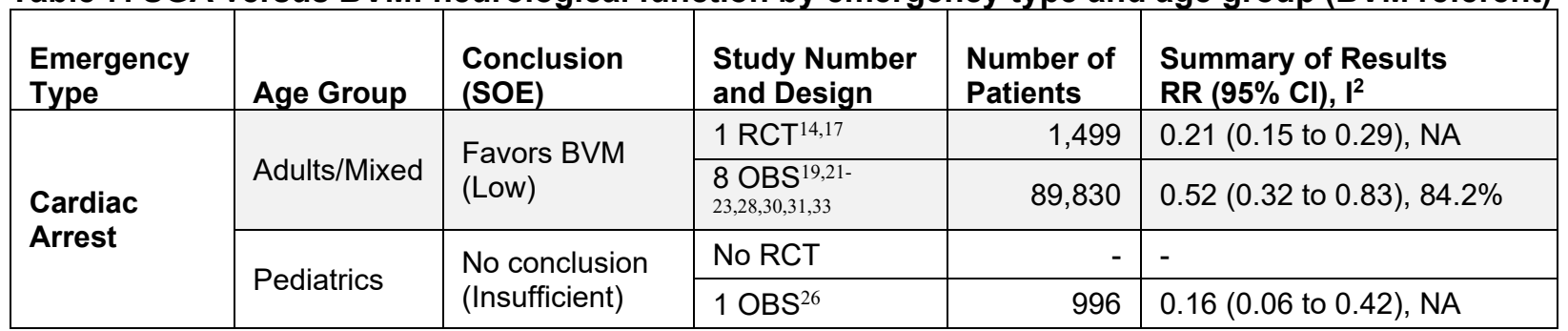

$\mathrm{BVM}=$ bag valve mask; $\mathrm{CI}=$ confidence interval; $\mathrm{I}^{2}=$ statistical test of heterogeneity; $\mathrm{NA}=$ not applicable; $\mathrm{OBS}=$ observational study; $\mathrm{RCT}$ = randomized controlled trial; $\mathrm{RR}=$ risk ratio; $\mathrm{SGA}=$ supraglottic airway; $\mathrm{SOE}=$ strength of evidence

\section{Return of Spontaneous Circulation}

Studies of ROSC were restricted to patients with cardiac arrest (Table 8). There was no difference in achieving any ROSC when comparing BVM versus SGA in RCTs and observational studies of adult patients (SOE: Low). For pediatric patients, SOE was insufficient, as there was only one eligible study. When the data were analyzed using unadjusted results, results for an observational study enrolling pediatrics favored SGA (Appendix Figure I-7); no other changes in effect were detected across sensitivity analyses. 
Table 8. SGA versus BVM: ROSC by emergency type and age group (BVM referent)

\begin{tabular}{|c|c|c|c|c|c|}
\hline $\begin{array}{l}\text { Emergency } \\
\text { Type }\end{array}$ & $\begin{array}{l}\text { Age } \\
\text { Group }\end{array}$ & $\begin{array}{l}\text { Conclusion } \\
\text { (SOE) }\end{array}$ & $\begin{array}{l}\text { Study Number and } \\
\text { Design }\end{array}$ & $\begin{array}{l}\text { Number of } \\
\text { Patients }\end{array}$ & $\begin{array}{l}\text { Summary of Results } \\
\text { RR }(95 \% \mathrm{Cl}), \mathrm{I}^{2}\end{array}$ \\
\hline \multirow{4}{*}{$\begin{array}{l}\text { Cardiac } \\
\text { Arrest }\end{array}$} & \multirow{2}{*}{ Adults } & \multirow{2}{*}{$\begin{array}{l}\text { No difference } \\
\text { (Low) }\end{array}$} & $3 \mathrm{RCT}^{13-15,17}$ & 1,657 & 0.94 (0.76 to 1.27$), 0 \%$ \\
\hline & & & $9 \mathrm{OBS}^{19-23,28,29,33,34}$ & 45,188 & 1.07 (0.82 to 1.40$), 87.2 \%$ \\
\hline & \multirow{2}{*}{ Pediatrics } & \multirow{2}{*}{$\begin{array}{l}\text { No conclusion } \\
\text { (Insufficient) }\end{array}$} & No RCT & - & - \\
\hline & & & $1 \mathrm{OBS}^{26}$ & 996 & 1.19 (0.75 to 1.88$), \mathrm{NA}$ \\
\hline
\end{tabular}

$\mathrm{BVM}$ = bag valve mask; $\mathrm{CI}=$ confidence interval; $\mathrm{I}^{2}=$ statistical test of heterogeneity; $\mathrm{NA}=$ not applicable; OBS = observational study; RCT = randomized controlled trial; ROSC = return of spontaneous circulation; $\mathrm{RR}=$ risk ratio; SGA = supraglottic airway; $\mathrm{SOE}=$ strength of evidence

\section{Qualitative Synthesis: Additional Outcomes}

Outcomes for comparisons of BVM to SGA that were not meta-analyzed included length of stay, measures of oxygenation/ventilation, and harms. These results are presented in Table 9. Overall, harms were similar between BVM and SGA groups across four studies (SOE: Moderate). ${ }^{13,15,18,19}$ There was no difference between BVM and SGA when comparing oxygenation and ventilation on emergency department (ED) arrival in five studies (SOE: Moderate) ${ }^{13,16,20,21,34}$ but one study ${ }^{20}$ reported lower arterial $\mathrm{pH}$ in the BVM group. For successful airway placement, results were inconsistent between studies with insufficient evidence to support a conclusion. Finally, for length of stay, only one observational study reported data (SOE: Insufficient). ${ }^{27}$

Table 9. BVM versus SGA: additional outcomes

\begin{tabular}{|c|c|c|c|c|}
\hline Outcome & $\begin{array}{l}\text { Conclusion } \\
\text { (SOE) }\end{array}$ & $\begin{array}{l}\text { Study Number } \\
\text { and Design }\end{array}$ & $\begin{array}{l}\text { Number } \\
\text { of } \\
\text { Patients }\end{array}$ & Summary of Findings \\
\hline Length of Stay & $\begin{array}{l}\text { No conclusion } \\
\text { (Insufficient) }\end{array}$ & $1 \mathrm{OBS}^{27}$ & 50 & $\begin{array}{l}\text { Significant difference in hospital- (6 } \\
\text { vs. } 1) \text { and ICU-free days favoring } \\
\text { BVM (7 vs. } 1), p<0.05\end{array}$ \\
\hline \multirow{2}{*}{$\begin{array}{l}\text { Successful } \\
\text { Airway }\end{array}$} & \multirow[b]{2}{*}{$\begin{array}{l}\text { No conclusion } \\
\text { (Insufficient) }\end{array}$} & $1 \mathrm{RCT}^{13}$ & 76 & No difference \\
\hline & & $1 \mathrm{OBS}^{19}$ & 517 & $\begin{array}{l}\text { Significantly fewer successful } \\
\text { airways established in BVM vs. } \\
\text { SGA }(30 \% \text { vs. } 93 \%, p<0.01)\end{array}$ \\
\hline \multirow[b]{2}{*}{$\begin{array}{l}\text { Oxygenation / } \\
\text { Ventilation }\end{array}$} & \multirow[b]{2}{*}{$\begin{array}{l}\text { No difference } \\
\text { (Moderate) }\end{array}$} & $2 \mathrm{RCT}^{13,16}$ & 145 & No difference \\
\hline & & $3 \mathrm{OBS}^{20,21,34}$ & 2,380 & $\begin{array}{l}\text { In } 1 \text { study, }{ }^{20} \text { significantly lower } \\
\text { median arterial pH in BVM vs. } \\
\text { SGA (7.08 mm Hg vs. } 7.12 \mathrm{~mm} \\
\mathrm{Hg}, \mathrm{p}=0.02)\end{array}$ \\
\hline \multirow[b]{2}{*}{ Harms } & \multirow[b]{2}{*}{$\begin{array}{l}\text { No difference } \\
\text { (Moderate) }\end{array}$} & $2 \mathrm{RCT}^{13,15}$ & 116 & $\begin{array}{l}\text { No differences between groups } \\
\text { across all reported harms }\end{array}$ \\
\hline & & $2 \mathrm{OBS}^{18,19}$ & 580 & $\begin{array}{l}\text { In } 1 \text { study, }{ }^{18} \text { lower rates of } \\
\text { aspiration pneumonitis within } 72 \\
\text { hours for BVM vs. SGA ( } 5 \% \text { vs. } \\
33 \% \text { ) }\end{array}$ \\
\hline
\end{tabular}

$\mathrm{BVM}=$ bag valve mask; ICU = intensive care unit; $\mathrm{OBS}=$ observational study; $\mathrm{RCT}=$ randomized controlled trial; $\mathrm{SGA}=$ supraglottic airway; $\mathrm{SOE}=$ strength of evidence 


\section{Key Question 2: Bag Valve Mask Compared With Endotracheal Intubation}

\section{Key Results}

When BVM was compared with ETI for prehospital airway management across all the included studies:

- Survival in-hospital or at 1 month (18 studies; $\mathrm{N}=52,770)$ was not significantly different for:

- Adult/mixed-age cardiac arrest patients (SOE: Moderate)

- Adult/mixed-age trauma patients (SOE: Low)

- Pediatric cardiac arrest patients (SOE: Low)

- Neurological function at discharge or 1-month post incident was not significantly different ( 9 studies; $\mathrm{N}=78,576$ ) for:

- Adult cardiac arrest patients (SOE: Moderate)

- Pediatric cardiac arrest patients (SOE: Low)

- ROSC rates were not significantly different (11 studies; $\mathrm{N}=48,802$ ) for:

- Adult cardiac arrest patients (SOE: Low)

- Pediatric cardiac arrest patients (SOE: Low)

- Harms: no differences (5 studies; $\mathrm{N}=3,918$; SOE: Moderate)

\section{Summary of Results}

We identified and analyzed 22 studies reported in 24 publications that compared patient outcomes for BVM and ETI (See Appendix C for the list of included studies). Two studies were reported in a single publication, ${ }^{24}$ while results from three studies were reported in two publications each. 35,36 and 37,38 and 14,17 These studies included three RCTs, ${ }^{14,17,37-39}$ one controlled clinical trial (CCT) which was included with the RCTs in the meta analyses, ${ }^{35,36}$ three prospective cohorts, ${ }^{18,21,22}$ and 15 retrospective cohorts. ${ }^{23-26,28-33,40-43}$

Seven of these studies were rated low ROB, ${ }^{23,25,26,31,37-39,42}$ six moderate ${ }^{24,29,32,33,41}$ and three high. ${ }^{30,40,43}$ Three were rated low ROB for survival and moderate for ROSC or neurological function outcomes. ${ }^{21,28,35,36}$ One study was rated moderate ROB for short-term outcomes and high ROB for survival at 1 month). ${ }^{22}$ (See Appendix G for ROB details).

The 22 studies included 106,325 patients and the individual studies ranged in size from $78^{30}$ to 49,534 patients. ${ }^{31}$ Most of the studies (15 of 22) included multiple emergency medical services (EMS) agencies or ambulance services. ${ }^{14,17,18,21,23,24,26,28,31,32,35-40,43}$ However, less than half (10 studies) were conducted in the United States, or the United States and Canada; ${ }^{14,17,18,24-26,35,36,40-43}$ nine were conducted in Asia (Japan, South Korea, Taiwan, Thailand); $22,23,28-33,43$ two in Europe, ${ }^{21,37,38}$ and one in Australia. ${ }^{39}$

\section{Meta-Analysis}

Meta analyses were conducted of studies that compared BVM to ETI for prehospital airway management for outcomes of survival, neurological function, and ROSC. Analyses were stratified by study design, age group and emergency type. For Key Question 2, sensitivity analyses did not change any conclusions. Therefore, only the primary analyses were summarized in this section. 


\section{Survival}

Eighteen studies contained data on survival for Key Question 2 comparing BVM and ETI, including 4 RCTs $^{14,17,35-37,39}$ and 14 observational studies. ${ }^{18,21-26,28,30,32,40-42}$

Across age groups and emergency types, the findings showed no difference in survival inhospital or at 1-month post incident when comparing BVM to ETI for prehospital airway management (Table 10; Appendix Figure H-4). For adult/mixed age cardiac arrest patients, we rated the SOE as moderate for this finding of no difference because the pooled estimates are based on several studies that included a large number of patients. The SOE was moderate rather than high because the findings were inconsistent across studies and the pooled estimates were not precise. We assessed the SOE as low for the conclusion of no difference for pediatric cardiac arrest patients and adult/mixed age trauma patients because each of these strata contained three studies with inconsistent results, and their pooled estimates were also imprecise. In both of these patient groups an RCT or CCT found no difference. The findings from the larger observational studies that favored BVM are likely affected by the fact that patients requiring ETI outside of controlled trials are less likely to survive. This suggests that future research could change the conclusion for these two groups.

For pediatric cardiac arrest patients, a moderate sized CCT $(n=591)^{35,36}$ and a small observational study of 136 patients found no difference, ${ }^{18}$ while a larger observational study $(n=1,508)$ found that survival was better with BVM. ${ }^{26}$ In the case of adult trauma patients, an RCT with 299 patients reported no difference ${ }^{39}$ while two observational studies involving 1,029 patients when combined favored BVM, reporting lower rates of survival with ETI. ${ }^{41,42}$ As only one retrospective cohort study of pediatric trauma patients was identified that reported survival, and it was rated as high risk of bias, ${ }^{40}$ the evidence was insufficient to support a conclusion.

Table 10. ETI versus BVM: survival by emergency type and age groups (BVM referent)

\begin{tabular}{|c|c|c|c|c|c|}
\hline $\begin{array}{l}\text { Emergency } \\
\text { Type }\end{array}$ & Age Group & $\begin{array}{l}\text { Conclusion } \\
\text { (SOE) }\end{array}$ & $\begin{array}{l}\text { Study Number } \\
\text { and Design }\end{array}$ & $\begin{array}{l}\text { Number of } \\
\text { Patients }\end{array}$ & $\begin{array}{l}\text { Summary of Results RR } \\
(95 \% \mathrm{Cl}), \mathrm{I}^{2}\end{array}$ \\
\hline \multirow{4}{*}{$\begin{array}{l}\text { Cardiac } \\
\text { Arrest }\end{array}$} & \multirow{2}{*}{ Adults/Mixed } & \multirow{2}{*}{$\begin{array}{l}\text { No difference } \\
\text { (Moderate) }\end{array}$} & $2 \mathrm{RCT}^{14,17,37,38}$ & 3,388 & 0.45 (0.07 to 2.89 ), $97.5 \%$ \\
\hline & & & 9 OBS $^{21-25,28,30,32}$ & 45,241 & 0.84 (0.52 to 1.32$), 85.4 \%$ \\
\hline & \multirow{2}{*}{ Pediatrics } & \multirow{2}{*}{$\begin{array}{l}\text { No difference } \\
\text { (Low) }\end{array}$} & $1 \mathrm{CCT}^{35,36}$ & 591 & $0.96(0.56$ to 1.66$), \mathrm{NA}$ \\
\hline & & & $2 \mathrm{OBS}^{18,26}$ & 1,644 & $0.44(0.28$ to 0.74$), 0 \%$ \\
\hline \multirow{4}{*}{ Trauma } & \multirow{2}{*}{ Adults/Mixed } & \multirow{2}{*}{$\begin{array}{l}\text { No difference } \\
\text { (Low) }\end{array}$} & $1 \mathrm{RCT}^{39}$ & 299 & 1.00 (0.85 to 1.16$), \mathrm{NA}$ \\
\hline & & & $2 \mathrm{OBS}^{41,42}$ & 1029 & $0.17(0.12$ to 0.32$), 10.7 \%$ \\
\hline & \multirow{2}{*}{ Pediatrics } & \multirow{2}{*}{$\begin{array}{l}\text { No conclusion } \\
\text { (Insufficient) }\end{array}$} & No RCT & - & - \\
\hline & & & $1 \mathrm{OBS}^{40}$ & 578 & $1.01(0.82$ to 1.24$), \mathrm{NA}$ \\
\hline
\end{tabular}

$\mathrm{BVM}=$ bag valve mask; $\mathrm{CCT}=$ controlled clinical trial; $\mathrm{CI}=$ confidence interval; $\mathrm{ETI}=$ endotracheal intubation; $\mathrm{NA}=$ not applicable; OBS = observational study; $\mathrm{RCT}=$ randomized controlled trial; $\mathrm{RR}=$ risk ratio; $\mathrm{SOE}=$ strength of evidence

\section{Neurological Function}

Data on neurological function were available from nine studies, including one RCT, ${ }^{37}$ one $\mathrm{CCT},{ }^{35,36}$ and seven observational studies. ${ }^{21-23,26,28,31,33}$ All of these studies involved cardiac arrest patients; none of the included studies of trauma patients included data on neurological function. The results for neurological function at discharge or 1-month post incident are presented in Table 11 and the forest plot in Appendix Figure H-5. 
The overall conclusion was of no difference when BVM was compared to ETI. For adults the evidence consisted of 7 studies with 76,477 patients. The SOE was moderate, not high, despite the large number of patients, because the findings were inconsistent across studies. One $\mathrm{RCT}^{37}$ and three observational studies ${ }^{22,23,28}$ reported no difference, while three other observational studies found that function was better with BVM when compared to ETI. ${ }^{21,31,33}$

For pediatric cardiac arrest patients, we made a conservative conclusion based on the fact that the intention-to-treat analysis of a clinical trial found no difference, ${ }^{35,36}$ while a single retrospective cohort study favored $\mathrm{BVM},{ }^{26}$ therefore there was some inconsistency. The SOE for the conclusion of no difference was low as we expect future studies could change this conclusion.

Table 11. ETI versus BVM: neurological function by emergency types and age groups (BVM referent)

\begin{tabular}{|c|c|c|c|c|c|}
\hline $\begin{array}{l}\text { Emergency } \\
\text { Type }\end{array}$ & $\begin{array}{l}\text { Age } \\
\text { Group }\end{array}$ & $\begin{array}{l}\text { Conclusion } \\
\text { (SOE) }\end{array}$ & $\begin{array}{l}\text { Study Number } \\
\text { and Design }\end{array}$ & $\begin{array}{l}\text { Number of } \\
\text { Patients }\end{array}$ & $\begin{array}{l}\text { Summary of Results RR } \\
(95 \% \mathrm{Cl}), \mathrm{I}^{2}\end{array}$ \\
\hline \multirow{4}{*}{$\begin{array}{l}\text { Cardiac } \\
\text { Arrest }\end{array}$} & \multirow{2}{*}{ Adults } & \multirow{2}{*}{$\begin{array}{l}\text { No difference } \\
\text { (Moderate) }\end{array}$} & $1 \mathrm{RCT}^{37,38}$ & 2,040 & 0.97 (0.65 to 1.47$), \mathrm{NA}$ \\
\hline & & & 6 OBS $^{21-23,28,31,33}$ & 74,437 & 0.65 (0.32 to 1.13$), 84.1 \%$ \\
\hline & \multirow{2}{*}{ Pediatrics } & \multirow{2}{*}{$\begin{array}{l}\text { No difference } \\
\text { (Low) }\end{array}$} & $1 \mathrm{CCT}^{35,36}$ & 591 & 1.45 (0.66 to 3.16$)$, NA \\
\hline & & & $1 \mathrm{OBS}^{26}$ & 1,508 & 0.33 (0.20 to 0.53 ), NA \\
\hline
\end{tabular}

$\mathrm{BVM}=$ bag valve mask; $\mathrm{CCT}=$ controlled clinical trial; $\mathrm{CI}=$ confidence interval; $\mathrm{ETI}=$ endotracheal intubation; $\mathrm{NA}=$ not applicable; $\mathrm{OBS}=$ observational study; $\mathrm{RCT}=$ randomized controlled trial; $\mathrm{RR}=$ risk ratio; $\mathrm{SOE}=$ strength of evidence Bold Text $=$ Moderate SOE

\section{Return of Spontaneous Circulation}

A total of 11 studies compared BVM and ETI in terms of ROSC for out-of-hospital cardiac arrest patients. The pooled results are summarized in Table 12 and the forest plot provided in Appendix Figure H-6. These studies include two RCTs ${ }^{14,17,37}$ and nine observational studies. ${ }^{21-}$ $23,25,26,28,29,33,43$ One study consisted of pediatric patients ${ }^{26}$ and the rest are of adults.

The studies comparing ROSC in adults include a RCT with low ROB conducted in France and Belgium. ${ }^{37}$ It is important to note that the providers performing ETI in this study were physicians, which limits generalizability to EMS systems in which nonphysicians perform ETI. Furthermore, it is unclear what level of provider provided BVM. This RCT and three of the observational studies ${ }^{22,23,28}$ favored ETI while one $\mathrm{RCT}^{14,17}$ and the other observational studies found no significant difference in ROSC for BVM versus ETI. The SOE was low.

Table 12. ETI versus BVM: ROSC by emergency type and age groups (BVM referent)

\begin{tabular}{|c|c|c|c|c|c|}
\hline $\begin{array}{l}\text { Emergency } \\
\text { Type }\end{array}$ & $\begin{array}{l}\text { Age } \\
\text { Group }\end{array}$ & $\begin{array}{l}\text { Conclusion } \\
\text { (SOE) }\end{array}$ & $\begin{array}{l}\text { Study Number and } \\
\text { Design }\end{array}$ & $\begin{array}{l}\text { Number of } \\
\text { Patients }\end{array}$ & $\begin{array}{l}\text { Summary of Results } \\
\text { RR }(95 \% \mathrm{Cl}), \mathrm{I}^{2}\end{array}$ \\
\hline \multirow{4}{*}{$\begin{array}{l}\text { Cardiac } \\
\text { Arrest }\end{array}$} & \multirow{2}{*}{ Adults } & \multirow{2}{*}{$\begin{array}{l}\text { No difference } \\
\text { (Low) }\end{array}$} & $2 \mathrm{RCT}^{14,17,37,38}$ & 3,338 & $\begin{array}{l}1.01(0.72 \text { to } 1,38) \\
68.9 \%\end{array}$ \\
\hline & & & 8 OBS $^{21-23,25,28,29,33,43}$ & 43,906 & $\begin{array}{l}1.18(0.85 \text { to } 1.56), \\
86.4 \%\end{array}$ \\
\hline & \multirow{2}{*}{ Pediatrics } & \multirow{2}{*}{$\begin{array}{l}\text { No difference } \\
\text { (Low) }\end{array}$} & No RCT & - & - \\
\hline & & & $1 \mathrm{OBS}^{26}$ & 1,508 & $1.10(0.88$ to 1.39$), \mathrm{NA}$ \\
\hline
\end{tabular}

$\mathrm{BVM}=$ bag valve mask; $\mathrm{CI}=$ confidence interval; $\mathrm{ETI}=$ endotracheal intubation; $\mathrm{NA}=$ not applicable; OBS = observational study; $\mathrm{RCT}=$ randomized controlled trial; $\mathrm{ROSC}=$ return of spontaneous circulation; $\mathrm{RR}=$ risk ratio; $\mathrm{SOE}=$ strength of evidence 


\section{Qualitative Synthesis: Additional Outcomes}

Outcomes from studies comparing BVM to ETI that were not meta-analyzed included length of stay ( 2 studies $\left.^{35,36,39}\right)$, successful airway insertion $\left(2\right.$ studies $\left.^{35-37}\right)$, and measures of adequate ventilation ( 2 studies $^{21,39}$ ). Additionally, four studies ${ }^{35-40}$ reported comparisons of several harms including the need for an additional airway, aspiration, oral trauma, vomiting/ regurgitation, and pneumothorax, as well as overall complications. Results are presented in Table 13. The SOE was low or insufficient for all of these additional outcomes except for harms. The four studies used different definitions of harms. Three of the four reported differences that were not statistically significant. The one significant finding was that regurgitation of gastric contents was 7.7 percentage points lower with ETI in one RCT.

Table 13. BVM versus ETI: additional outcomes

\begin{tabular}{|c|c|c|c|c|}
\hline Outcome & $\begin{array}{l}\text { Conclusion } \\
\text { (SOE) }\end{array}$ & $\begin{array}{l}\text { Number of } \\
\text { Studies }\end{array}$ & $\begin{array}{l}\text { Number of } \\
\text { Patients }\end{array}$ & Summary of Findings \\
\hline Length of Stay & $\begin{array}{l}\text { No difference } \\
\text { (Low) }\end{array}$ & $\begin{array}{l}1 \mathrm{RCT}^{39} \\
1 \mathrm{CCT}^{35,36}\end{array}$ & 1,142 & $\begin{array}{l}\text { - No significant difference in } \\
\text { median total days in hospital in } \\
\text { both studies } \\
\text { - No significant different in time } \\
\text { in ICU in both studies }\end{array}$ \\
\hline $\begin{array}{l}\text { Successful } \\
\text { Airway }\end{array}$ & $\begin{array}{l}\text { No conclusion } \\
\text { (Insufficient) }\end{array}$ & $\begin{array}{l}1 \mathrm{RCT}^{37} \\
1 \mathrm{CCT}^{35,36}\end{array}$ & 2,873 & $\begin{array}{l}\text { Inconsistent findings from studies } \\
\text { with different analytic approaches }\end{array}$ \\
\hline \multirow[t]{2}{*}{$\begin{array}{l}\text { Oxygenation / } \\
\text { Ventilation }\end{array}$} & \multirow[t]{2}{*}{$\begin{array}{l}\text { No difference } \\
\text { (Low) }\end{array}$} & $1 \mathrm{RCT}^{39}$ & 160 & $\begin{array}{l}\text { - No statistical difference in } \\
\mathrm{SaO}_{2}, \mathrm{pH}, \mathrm{PaO}_{2}, \mathrm{PaCO}_{2} \text {, at ED } \\
\text { arrival or Initial } \mathrm{ETCO}_{2}\end{array}$ \\
\hline & & $1 \mathrm{OBS}^{21}$ & 793 & $\begin{array}{l}\text { No clinical difference in initial } \\
\mathrm{ETCO}_{2} \text { or } \mathrm{SpO}_{2} \text { in propensity } \\
\text { matched groups and no } \\
\text { difference in } \mathrm{SpO}_{2} \text { in the total } \\
\text { cohort }\end{array}$ \\
\hline \multirow[t]{2}{*}{ Harms } & \multirow[t]{2}{*}{$\begin{array}{l}\text { No difference } \\
\text { (Moderate) }\end{array}$} & $\begin{array}{l}2 \mathrm{RCTs}^{37-39} \\
1 \mathrm{CCT}^{35,36}\end{array}$ & 3,062 & $\begin{array}{l}\text { - No difference in } \\
\text { ○ need for additional } \\
\text { airway } \\
\text { ○ aspiration, oral/airway } \\
\text { trauma or vomiting } \\
\text { o pneumothorax } \\
\text { - ETI resulted in expected lower } \\
\text { regurgitation of gastric } \\
\text { contents } 15.2 \% \text { vs. } 7.5 \% \text {; } \\
\text { absolute difference } 7.7 \%(95 \% \\
\mathrm{Cl} 4.9 \text { to } 10.4), \mathrm{p}<0.001\end{array}$ \\
\hline & & $2 \mathrm{OBS}^{18,40}$ & 714 & $\begin{array}{l}\text { - No difference in broadly } \\
\text { defined complications } \\
\text { - No different in blood in airway, } \\
\text { swelling, or pharyngeal injury }\end{array}$ \\
\hline
\end{tabular}

$\mathrm{BVM}=$ bag valve mask; ETI = endotracheal intubation; ICU = intensive care unit; OBS = observational study; RCT = randomized controlled trial; $\mathrm{SGA}=$ supraglottic airway; $\mathrm{SOE}=$ strength of evidence

Studies that reported results for survival and neurological function that could not be included in the meta-analyses are summarized in Table 14. Five studies reported survival to hospital admission, which was not clinically similar enough to be combined with the other time points of survival to discharge or 1-month post injury in meta-analysis. However, the conclusion remains the same as all reported no difference in survival rates between BVM and ETI. ${ }^{14,17,25,26,32,37}$ Three studies reported neurological function using three different measures (Glasgow Outcome Scale $[\mathrm{GOS}],{ }^{30}$ Functional Independence Measure $[\mathrm{FIM}],{ }^{40}$ and $\left.\mathrm{mRS}\right) .{ }^{14,17}$ The study results were not 
pooled due to this difference in assessment method. Given the inconsistent results across studies, we assessed this evidence as insufficient to support a conclusion.

Table 14. BVM versus ETI: additional studies of survival and neurological function

\begin{tabular}{|c|c|c|c|c|}
\hline Outcome & $\begin{array}{l}\text { Conclusion } \\
\text { (SOE) }\end{array}$ & $\begin{array}{l}\text { Number of } \\
\text { Studies }\end{array}$ & $\begin{array}{l}\text { Number of } \\
\text { Patients }\end{array}$ & Summary of Findings \\
\hline \multirow{2}{*}{$\begin{array}{l}\text { Survival to } \\
\text { Hospital } \\
\text { Admission }\end{array}$} & \multirow{2}{*}{$\begin{array}{l}\text { No difference } \\
\text { (Low) }\end{array}$} & $2 \mathrm{RCT}^{14,17,37}$ & 3,978 & - No significant difference \\
\hline & & $3 \mathrm{OBS}^{25,26,32}$ & 3,162 & - No significant difference \\
\hline \multirow{2}{*}{$\begin{array}{l}\text { Neurological } \\
\text { Function }\end{array}$} & \multirow{2}{*}{$\begin{array}{l}\text { No conclusion } \\
\text { (Insufficient) }\end{array}$} & $1 \mathrm{RCT}^{14,17}$ & 1,348 & \multirow{2}{*}{$\begin{array}{l}\text { Inconsistent finding from } 3 \\
\text { studies using different measures } \\
\text { of function (GOS, FIM, mRS) }\end{array}$} \\
\hline & & $2 \mathrm{OBS}^{30,40}$ & 211 & \\
\hline
\end{tabular}

$\mathrm{BVM}=$ bag valve mask; ETI = endotracheal intubation; FIM = Functional Independence Measure; GOS = Glasgow Outcome Scale; OBS = observational study; $\mathrm{mRS}=$ modified Rankin Scale; RCT = randomized controlled trial; $\mathrm{SOE}=\mathrm{strength}$ of evidence

\section{Key Question 3: Supraglottic Airway Compared With Endotracheal Intubation}

\section{Key Results}

When SGA was compared with ETI for prehospital airway management:

- Survival in-hospital or at 1-month post incident (20 studies; $N=180,692$ ) was not significantly different between SGA and ETI for:

- Adult/mixed-age cardiac arrest patients (SOE: Low)

- Pediatric cardiac arrest patients (SOE: Low)

- Neurological function, defined as good neurological outcomes at discharge or 1-month post incident (16 studies; $\mathrm{N}=203,246$ ):

- Favored ETI versus SGA for adult cardiac arrest patients when measured by the CPC (SOE: Low)

$\circ$ Was not significantly different between SGA and ETI for adult cardiac arrest patients when measured by the mRS (SOE: Low)

- Was not significantly different between SGA and ETI for pediatric cardiac arrest patients when measured by the Pediatric Cerebral Performance Category (PCPC) (SOE: Low)

- Return of spontaneous circulation, defined as any ROSC (18 studies; N=186,642):

- Favored SGA over ETI for adult cardiac arrest patients (SOE: Low)

- Was not significantly different between SGA and ETI for Pediatric cardiac arrest patients (SOE: Low)

- First-pass success (9 studies; $\mathrm{N}=34,200$ ):

$\circ$ Favored SGA versus ETI for adult cardiac arrest patients, pediatric cardiac arrest patients, and adult patients with mixed emergency types (SOE: Low)

- Was not significantly different between SGA and ETI for adult medical emergency patients (SOE: Low)

- Overall success (14 studies; $\mathrm{N}=71,660$ ) was not significantly different between SGA and ETI for:

- Adult cardiac arrest patients (SOE: Moderate)

- Adult medical emergency patients (SOE: Moderate)

$\circ$ Adult patients with mixed emergency types (SOE: Moderate) 
- Harms (7 studies; $\mathrm{N}=13,687$ )

- No difference between SGA and ETI for aspiration (2 studies; SOE: Moderate)

- No difference between SGA and ETI for oral/airway trauma (2 studies; SOE: Moderate)

O No difference between SGA and ETI for regurgitation (single RCT; SOE: Moderate)

- Favored SGA for multiple insertion attempts (single RCT; SOE: Moderate)

- Favored ETI for inadequate ventilation (single RCT; SOE: Moderate)

\section{Summary of Results}

We identified and analyzed 41 studies that compared patient outcomes for SGA and ETI $(\mathrm{N}=383,953)$. One publication ${ }^{24}$ included two studies and two publications ${ }^{14,17}$ were reported as one study because the latter was a secondary analysis (See Appendix C for the list of included studies). These included 4 RCTs $,{ }^{14,17,44-46} 2 \mathrm{CCTs},{ }^{47,48} 9$ prospective cohorts, ${ }^{18,21,22,49-54} 25$ retrospective cohorts, ${ }^{23-26,28-33,55-68}$ and 1 before-after study. ${ }^{69}$

Ten of these studies were rated low ROB, ${ }^{23,25,26,31,44,46,54,64,68,69}$ fifteen moderate, ${ }^{18,24,29,32,33,45,48,50,51,53,57,59,60,62,63}$ and eight high. ${ }^{30,47,49,55,56,58,61,67}$ One study was rated low for one outcome and moderate for two other outcomes; ${ }^{28}$ one study was rated moderate for one outcome and high for one outcome; ${ }^{22}$ two studies were rated low for one outcome and moderate for one outcome; ${ }^{17,21}$ two studies were rated low for two outcomes and moderate for one, ${ }^{65,66}$ and one study was rated low for one outcome and high for one outcome (Appendix G) ${ }^{52}$

Sample sizes in the included studies ranged from 78 to 138,248 participants; 21 studies were conducted in the United States and Canada, ${ }^{17,18,24-26,45,47-49,51,54-57,61-63,67-69} 11$ in Asia, ${ }^{22,23,28-}$ $33,52,64,668$ in Europe, ${ }^{21,44,46,50,58-60,65}$ and 1 in Australia. ${ }^{53}$

\section{Meta-Analysis}

Meta-analysis was performed for six outcomes: survival in-hospital or at 1-month post incident; neurological function using modified Rankin Scale; neurological function using CPC or Pediatric Cerebral Performance Category (PCPC); ROSC; first-pass success; and overall success. Thirty-seven studies were included in meta-analysis for one or more of these outcomes. The results for each outcome by subgroup are reported in Tables 15 to 20, and forest plots are provided in Appendix Figures H-7 to H-11.

\section{Survival}

Twenty studies contained data on survival in-hospital or at 1-month post incident for Key Question 3 comparing SGA and ETI, including three RCTs ${ }^{14,17,44,46}$ and 17 observational studies $^{18,21-26,28,30,32,52,57,62,64-66}$ (Table 15). Of the 20 studies, 19 included patients with cardiac arrest, and $1^{57}$ included trauma patients.

For studies of adult/mixed-age cardiac arrest patients, pooled analysis of 3 RCTs found no difference in survival between airway interventions, while ETI was associated with higher rates of survival in 13 observational studies. Sensitivity analyses resulted in similar findings (Appendix Figures I-8 to I-9). Of the 13 observational studies, four favored ETI, including one very large retrospective cohort study $(n=138,248$ analyzed for this outcome) conducted in Japan; ${ }^{64}$ the other nine found no difference in survival. Seven of the 13 studies were conducted in Asia, where ETI is used less frequently than SGA in the prehospital setting, potentially introducing bias from provider skills or preference of airway. The overall conclusion was that 
there is no difference between SGA and ETI on survival in adult/mixed-age cardiac patients (SOE: Low).

In three observational studies of pediatric patients (SOE: Low). ${ }^{18,26,66}$ There was no difference in survival for SGA compared with ETI.

Table 15. ETI versus SGA: survival by emergency type and age group (SGA referent)

\begin{tabular}{|c|c|c|c|c|c|}
\hline $\begin{array}{l}\text { Emergency } \\
\text { Type }\end{array}$ & Age Group & $\begin{array}{l}\text { Conclusion } \\
\text { (SOE) }\end{array}$ & $\begin{array}{l}\text { Study Number and } \\
\text { Design }\end{array}$ & $\begin{array}{l}\text { Number of } \\
\text { Patients }\end{array}$ & $\begin{array}{l}\text { Summary of Results } \\
\text { RR }(95 \% \mathrm{Cl}), \mathrm{I}^{2}\end{array}$ \\
\hline \multirow{4}{*}{$\begin{array}{l}\text { Cardiac } \\
\text { Arrest }\end{array}$} & \multirow{2}{*}{ Adults/Mixed } & \multirow{2}{*}{$\begin{array}{l}\text { No } \\
\text { difference } \\
\text { (Low) }\end{array}$} & $3 \mathrm{RCT}^{14,17,44,46}$ & 12,465 & $\begin{array}{l}0.89(0.55 \text { to } 1.24) \\
54.4 \%\end{array}$ \\
\hline & & & $13 \mathrm{OBS}^{21-25,28,30,32,52,62,64,65}$ & 164,623 & $\begin{array}{l}1.24(1.12 \text { to } 1.45) \\
40.0 \%\end{array}$ \\
\hline & \multirow{2}{*}{ Pediatrics } & \multirow{2}{*}{$\begin{array}{l}\text { No } \\
\text { difference } \\
\text { (Low) }\end{array}$} & No RCT & - & - \\
\hline & & & $3 \mathrm{OBS}^{18,26,66}$ & 1,260 & $0.76(0.48$ to 1.23$), 0 \%$ \\
\hline \multirow{3}{*}{ Trauma } & \multirow{2}{*}{ Adults/Mixed } & \multirow{2}{*}{$\begin{array}{l}\text { No } \\
\text { conclusion } \\
\text { (Insufficient) }\end{array}$} & No RCT & - & - \\
\hline & & & $1 \mathrm{OBS}^{57}$ & 2,344 & 3.35 (2.40 to 4.68$)$, NA \\
\hline & Pediatrics & - & - & - & - \\
\hline
\end{tabular}

$\mathrm{CI}=$ confidence interval; ETI = endotracheal intubation; $\mathrm{I}^{2}=$ statistical test of heterogeneity; NA = not applicable; OBS = observational study; $\mathrm{RCT}=$ randomized controlled trial; $\mathrm{RR}=$ risk ratio; $\mathrm{SGA}=$ supraglottic airway; $\mathrm{SOE}=$ strength of evidence

\section{Neurological Function}

Sixteen studies (2 RCTs and 14 observational) contained data for pooled analysis of neurological function measured at discharge or 1-month post incident. Outcomes assessed by the mRS were analyzed separately from those assessed using the CPC or PCPC (mRS in Table 16, $\mathrm{CPC} / \mathrm{PCPC}$ in Table 17). All studies were in cardiac arrest patients.

\section{Modified Rankin Scale}

Three studies ( 2 RCTs $^{17,44}$ and 1 observational ${ }^{68}$ ) assessed neurological function using the $\mathrm{mRS}$ (good outcome $=\mathrm{mRS}$ score $0-3$ ); all were in adult patients. The pooled results of the two RCTs showed no difference; the observational study favored ETI. We performed sensitivity analyses and found higher rates of good neurological function in patients treated with SGA in analysis grouped by first type of airway received (Appendix Figures I-8 to I-9), ${ }^{44}$ rendering the findings from that study susceptible to indication bias. The overall conclusion was that there is likely no difference in neurological function between SGA and ETI, with low SOE due to inconsistency in findings between studies, and inconsistency in one RCT between primary and sensitivity analyses results. 
Table 16. ETI versus SGA: neurological function - modified Rankin Scale by emergency type and age group (SGA referent)

\begin{tabular}{|l|l|l|l|l|l|}
\hline $\begin{array}{l}\text { Emergency } \\
\text { Type }\end{array}$ & $\begin{array}{l}\text { Age } \\
\text { Group }\end{array}$ & $\begin{array}{l}\text { Conclusion } \\
\text { (SOE) }\end{array}$ & $\begin{array}{l}\text { Study Number } \\
\text { and Design }\end{array}$ & $\begin{array}{l}\text { Number of } \\
\text { Patients }\end{array}$ & $\begin{array}{l}\text { Summary of Results } \\
\text { RR (95\% Cl), } \mathbf{2}^{2}\end{array}$ \\
\hline \multirow{3}{*}{ Cardiac Arrest } & \multirow{2}{*}{$\begin{array}{l}\text { No difference } \\
\text { (Low) }\end{array}$} & 2 RCT $^{17,44}$ & 12,293 & $0.90(0.52$ to 1.47$), 68.6 \%$ \\
\cline { 2 - 6 } & & 1 OBS $^{68}$ & 10,455 & 1.38 (1.04 to 1.83), NA \\
\cline { 2 - 6 } & Pediatrics & - & - & - & - \\
\hline
\end{tabular}

$\mathrm{CI}=$ confidence interval; $\mathrm{CCT}=$ controlled clinical trial; $\mathrm{ETI}=$ endotracheal intubation; $\mathrm{I}^{2}=$ statistical test of heterogeneity; NA = not applicable; $\mathrm{OBS}$ = observational study; $\mathrm{RCT}$ = randomized controlled trial; $\mathrm{RR}$ = risk ratio; $\mathrm{SGA}$ = supraglottic airway; $\mathrm{SOE}=$ strength of evidence

\section{Cerebral Performance Category/Pediatric Cerebral Performance Category}

Thirteen studies assessed neurological function using the CPC or PCPC (good outcome $=$ CPC or PCPC score 1-2). ${ }^{21-23,26,28,31,33,52,59,62,64-66}$ All studies were observational. Pooled results slightly favored ETI over SGA in adults. However, of the 11 adult studies, only 2 favored ETI, and the remaining 9 showed no difference. There was no difference in pediatric patients (SOE: Low) (Table 17).

Table 17. ETI versus SGA: neurological function-Cerebral Performance Category/Pediatric Cerebral Performance Category by emergency type and age group (SGA referent)

\begin{tabular}{|c|c|c|c|c|c|}
\hline $\begin{array}{l}\text { Emergency } \\
\text { Type }\end{array}$ & $\begin{array}{l}\text { Age } \\
\text { Group }\end{array}$ & $\begin{array}{l}\text { Conclusion } \\
\text { (SOE) }\end{array}$ & $\begin{array}{l}\text { Study Number } \\
\text { and Design }\end{array}$ & $\begin{array}{l}\text { Number of } \\
\text { Patients }\end{array}$ & $\begin{array}{l}\text { Summary of Results } \\
\text { RR }(95 \% \mathrm{Cl}), \mathrm{I}^{2}\end{array}$ \\
\hline \multirow{4}{*}{$\begin{array}{l}\text { Cardiac } \\
\text { Arrest }\end{array}$} & \multirow{2}{*}{$\begin{array}{l}\text { Adults } \\
\text { CPC }\end{array}$} & \multirow{2}{*}{$\begin{array}{l}\text { Favors ETI } \\
\text { (Low) }\end{array}$} & No RCT & - & - \\
\hline & & & $\begin{array}{l}11 \mathrm{OBS}^{21-} \\
23,28,31,33,52,59,62,64,65\end{array}$ & 182,543 & 1.15 (1.02 to 1.28$), 0 \%$ \\
\hline & \multirow{2}{*}{$\begin{array}{l}\text { Pediatrics } \\
\text { PCPC }\end{array}$} & \multirow{2}{*}{$\begin{array}{l}\text { No difference } \\
\text { (Low) }\end{array}$} & No RCT & - & - \\
\hline & & & $2 \mathrm{OBS}^{26,66}$ & 1,168 & 0.82 (0.37 to 2.75 ), $0 \%$ \\
\hline
\end{tabular}

$\mathrm{CI}=$ confidence interval; $\mathrm{CCT}=$ controlled clinical trial; $\mathrm{CPC}=$ Cerebral Performance Category; $\mathrm{ETI}=$ endotracheal intubation; $\mathrm{I}^{2}$ = statistical test of heterogeneity; NA = not applicable; OBS = observational study; PCPC = Pediatric Cerebral Performance Category; $\mathrm{RCT}=$ randomized controlled trial; $\mathrm{RR}=$ risk ratio; $\mathrm{SGA}=$ supraglottic airway; $\mathrm{SOE}=$ strength of evidence

\section{Return of Spontaneous Circulation}

Eighteen studies provided data for pooled analysis of ROSC (Table 18). In adults, pooled estimates from combining three RCTs favored SGA. Pooling of 13 observational studies found ETI associated with higher rates of ROSC. The overall conclusion was that SGA was associated with higher rates of ROSC in adults, based on pooled results from the RCTs, with low SOE due to inconsistency in findings between RCTs and observational studies. There was no difference found in pediatric studies (SOE: Low).

Table 18. ETI versus SGA: ROSC by age group (SGA referent)

\begin{tabular}{|c|c|c|c|c|c|}
\hline $\begin{array}{l}\text { Emergency } \\
\text { Type }\end{array}$ & $\begin{array}{l}\text { Age } \\
\text { Group }\end{array}$ & $\begin{array}{l}\text { Conclusion } \\
\text { (SOE) }\end{array}$ & Study Number and Design & $\begin{array}{l}\text { Number of } \\
\text { Patients }\end{array}$ & $\begin{array}{l}\text { Summary of Results } \\
\text { RR }(95 \% \mathrm{Cl}), \mathrm{I}^{2}\end{array}$ \\
\hline \multirow{4}{*}{$\begin{array}{l}\text { Cardiac } \\
\text { Arrest }\end{array}$} & \multirow[b]{2}{*}{ Adults } & \multirow{2}{*}{$\begin{array}{l}\text { Favors SGA } \\
\text { (Low) }\end{array}$} & $3 \mathrm{RCT}^{17,44,46}$ & 12,460 & 0.91 (0.83 to 0.97$), 0 \%$ \\
\hline & & & $13 \mathrm{OBS}^{21-23,28,29,33,51,52,55,62,64,65,68}$ & 173,014 & $\begin{array}{l}1.41(1.29 \text { to } 1.53) \text {, } \\
66.7 \%\end{array}$ \\
\hline & \multirow{2}{*}{ Pediatrics } & \multirow{2}{*}{$\begin{array}{l}\text { No difference } \\
\text { (Low) }\end{array}$} & No RCT & - & - \\
\hline & & & $2 \mathrm{OBS}^{26,66}$ & 1,168 & 0.81 (0.56 to 1.70$), 0 \%$ \\
\hline
\end{tabular}

$\mathrm{CI}=$ confidence interval; ETI = endotracheal intubation; $\mathrm{I}^{2}=$ statistical test of heterogeneity; NA = not applicable; OBS = observational study; $\mathrm{RCT}=$ randomized controlled trial; $\mathrm{ROSC}=$ return of spontaneous circulation; $\mathrm{RR}=$ risk ratio; $\mathrm{SGA}=$ supraglottic airway; $\mathrm{SOE}=$ strength of evidence 


\section{Successful Insertion of Advanced Airway}

Nine studies provided data for pooled analysis of first-pass success (Table 19). In adults with cardiac arrest, results favored SGA over ETI in the single $\mathrm{RCT}^{17}$ and the four pooled observational studies ${ }^{49,51,61,69}$ (SOE: Low). In pediatric patients with cardiac arrest, results from two observational studies ${ }^{18,61}$ favored SGA (SOE: Low). In adults with medical emergencies, results of a single $\mathrm{RCT}^{45}$ indicated no difference, and results of one observational study ${ }^{61}$ favored SGA (SOE: Low). In adults with mixed emergency types, results of two observational studies $^{53,63}$ favored SGA (SOE: Low).

Table 19. ETI versus SGA: first-pass successful advanced airway insertion by emergency type and age group (SGA referent)

\begin{tabular}{|c|c|c|c|c|c|}
\hline $\begin{array}{l}\text { Emergency } \\
\text { Type }\end{array}$ & $\begin{array}{l}\text { Age } \\
\text { Group }\end{array}$ & $\begin{array}{l}\text { Conclusion } \\
\text { (SOE) }\end{array}$ & $\begin{array}{l}\text { Study Number } \\
\text { and Design }\end{array}$ & $\begin{array}{l}\text { Number of } \\
\text { Patients }\end{array}$ & $\begin{array}{l}\text { Summary of Results } \\
\text { RR }(95 \% \mathrm{Cl}), \mathbf{I}^{2}\end{array}$ \\
\hline \multirow{4}{*}{ Cardiac Arrest } & \multirow{2}{*}{ Adults } & \multirow{2}{*}{$\begin{array}{l}\text { Favors SGA } \\
\text { (Low) }\end{array}$} & $1 \mathrm{RCT}^{17}$ & 3,004 & 0.57 (0.54 to 0.60$)$, NA \\
\hline & & & $4 \mathrm{OBS}^{49,51,61,69}$ & 20,531 & 0.80 (0.78 to 0.86 ), $0 \%$ \\
\hline & \multirow{2}{*}{ Pediatrics } & \multirow{2}{*}{$\begin{array}{l}\text { Favors SGA } \\
\text { (Low) }\end{array}$} & No RCT & - & - \\
\hline & & & $2 \mathrm{OBS}^{18,61}$ & 445 & 0.67 (0.56 to 0.78$), 0 \%$ \\
\hline \multirow{4}{*}{ Trauma } & \multirow{2}{*}{ Adults } & \multirow{2}{*}{$\begin{array}{l}\text { No conclusion } \\
\text { (Insufficient) }\end{array}$} & No RCT & - & - \\
\hline & & & $1 \mathrm{OBS}^{61}$ & 2,143 & 0.79 (0.75 to 0.83$), \mathrm{NA}$ \\
\hline & \multirow{2}{*}{ Pediatrics } & \multirow{2}{*}{$\begin{array}{l}\text { No conclusion } \\
\text { (Insufficient) }\end{array}$} & No RCT & - & - \\
\hline & & & $1 \mathrm{OBS}^{61}$ & 69 & 0.67 (0.48 to 0.94$)$, NA \\
\hline \multirow{3}{*}{ Medical } & \multirow{2}{*}{ Adults } & \multirow{2}{*}{$\begin{array}{l}\text { No difference } \\
\text { (Low) }\end{array}$} & $1 \mathrm{RCT}^{45}$ & 204 & $0.99(0.81$ to 1.20$), \mathrm{NA}$ \\
\hline & & & $1 \mathrm{OBS}^{61}$ & 7,297 & 0.88 (0.85 to 0.91$), \mathrm{NA}$ \\
\hline & Pediatrics & $\begin{array}{l}\text { No conclusion } \\
\text { (Insufficient) }\end{array}$ & $1 \mathrm{OBS}^{61}$ & 100 & 0.76 (0.42 to 1.38 ), NA \\
\hline \multirow{3}{*}{$\begin{array}{l}\text { Mixed } \\
\text { Emergency } \\
\text { Types }\end{array}$} & \multirow{2}{*}{ Adults } & \multirow{2}{*}{$\begin{array}{l}\text { Favors SGA } \\
\text { (Low) }\end{array}$} & No RCT & - & - \\
\hline & & & $2 \mathrm{OBS}^{53,63}$ & 407 & 0.57 (0.47 to 0.79$), 0 \%$ \\
\hline & Pediatrics & - & - & - & - \\
\hline
\end{tabular}

$\mathrm{CI}=$ confidence interval; $\mathrm{ETI}=$ endotracheal intubation; $\mathrm{I}^{2}=$ statistical test of heterogeneity; $\mathrm{NA}=$ not applicable; $\mathrm{OBS}=$ observational study; $\mathrm{RCT}=$ randomized controlled trial; $\mathrm{RR}=$ risk ratio; $\mathrm{SGA}=$ supraglottic airway; $\mathrm{SOE}=$ strength of evidence

Fourteen studies were pooled for analysis of overall success rates for insertion of advanced airway (Table 20). ${ }^{14,17,44-46,48-51,53,54,56,58,67,69}$ When ETI and SGA were compared, there were no differences in overall success rates for any subgroups (SOE: Moderate).

Table 20. ETI versus SGA: overall successful advanced airway insertion by emergency type and age group (SGA referent)

\begin{tabular}{|c|c|c|c|c|c|}
\hline $\begin{array}{l}\text { Emergency } \\
\text { Type }\end{array}$ & $\begin{array}{l}\text { Age } \\
\text { Group }\end{array}$ & $\begin{array}{l}\text { Conclusion } \\
\text { (SOE) }\end{array}$ & $\begin{array}{l}\text { Study Number } \\
\text { and Design }\end{array}$ & $\begin{array}{l}\text { Number of } \\
\text { Patients }\end{array}$ & $\begin{array}{l}\text { Summary of Results } \\
\text { RR }(95 \% \mathrm{Cl}), \mathrm{I}^{2}\end{array}$ \\
\hline \multirow{3}{*}{ Cardiac Arrest } & \multirow{2}{*}{ Adults } & \multirow{2}{*}{$\begin{array}{l}\text { No difference } \\
\text { (Moderate) }\end{array}$} & $3 \mathrm{RCT}^{14,17,44,46}$ & 11,720 & 0.94 (0.89 to 1.00 ), $77.9 \%$ \\
\hline & & & 6 OBS $^{49-51,58,67,69}$ & 36,983 & 0.94 (0.85 to 1.04 ), $90.0 \%$ \\
\hline & Pediatrics & - & - & - & - \\
\hline \multirow{3}{*}{ Trauma } & \multirow{2}{*}{ Adults } & \multirow{2}{*}{$\begin{array}{l}\text { No } \\
\text { conclusion } \\
\text { (Insufficient) }\end{array}$} & No RCT & - & - \\
\hline & & & $1 \mathrm{OBS}^{67}$ & 3,842 & 0.93 (0.88 to 0.98$), \mathrm{NA}$ \\
\hline & Pediatrics & - & - & - & - \\
\hline
\end{tabular}




\begin{tabular}{|c|c|c|c|c|c|}
\hline $\begin{array}{l}\text { Emergency } \\
\text { Type }\end{array}$ & $\begin{array}{l}\text { Age } \\
\text { Group }\end{array}$ & $\begin{array}{l}\text { Conclusion } \\
\text { (SOE) }\end{array}$ & $\begin{array}{l}\text { Study Number } \\
\text { and Design }\end{array}$ & $\begin{array}{l}\text { Number of } \\
\text { Patients }\end{array}$ & $\begin{array}{l}\text { Summary of Results } \\
\text { RR }(95 \% \mathrm{Cl}), \mathbf{I}^{2}\end{array}$ \\
\hline \multirow{3}{*}{ Medical } & \multirow{2}{*}{ Adults } & \multirow{2}{*}{$\begin{array}{l}\text { No difference } \\
\text { (Moderate) }\end{array}$} & $1 \mathrm{RCT}^{45}$ & 204 & $1.00(0.87$ to 1.15$), \mathrm{NA}$ \\
\hline & & & $2 \mathrm{OBS}^{48,67}$ & 15,644 & $0.90(0.86$ to 1.08$), 0 \%$ \\
\hline & Pediatrics & - & - & - & - \\
\hline \multirow{3}{*}{$\begin{array}{l}\text { Mixed } \\
\text { Emergency } \\
\text { Types }\end{array}$} & \multirow{2}{*}{ Adults } & \multirow{2}{*}{$\begin{array}{l}\text { No difference } \\
\text { (Moderate) }\end{array}$} & No RCT & - & - \\
\hline & & & $3 \mathrm{OBS}^{53,54,56}$ & 3,267 & $1.02(0.99$ to 1.06$), 0 \%$ \\
\hline & Pediatrics & - & - & - & - \\
\hline
\end{tabular}

$\mathrm{CI}=$ confidence interval; $\mathrm{ETI}=$ endotracheal intubation; $\mathrm{I}^{2}=$ statistical test of heterogeneity; NA $=$ not applicable; OBS $=$ observational study; $\mathrm{RCT}=$ randomized controlled trial; $\mathrm{RR}=$ risk ratio; $\mathrm{SGA}=$ supraglottic airway; $\mathrm{SOE}=$ strength of evidence

\section{Qualitative Synthesis: Additional Outcomes}

Included studies reported on other outcomes in addition to those synthesized with metaanalyses reported above, and these are summarized and qualitatively synthesized below (Table 21). These outcomes include survival to other time points ( 24 hours, 72 hours, and to ED, hospital, or intensive care unit [ICU] admission); ${ }^{14,25,26,32,44,46,49,55,62,65,68}$ neurological function measured by the GOS; ${ }^{30}$ various measures of oxygenation or ventilation efficacy; ${ }^{21,47,55,59}$ and harms, including aspiration, regurgitation, failure of airway insertion (requiring multiple attempts), inadequate ventilation, oral/airway trauma, dislodgment or unrecognized misplacement, and other complications. ${ }^{17,18,44,49,54,63,69}$ No studies provided results for length of stay (hospital or ICU), or morbidity (pneumothorax or aspiration pneumonia).

No difference between SGA and ETI was found for 72-hour survival; survival to ED, hospital, or ICU admission; and oxygenation/ventilation. For harms, no difference between SGA and ETI was found for aspiration, oral/airway trauma, and regurgitation (SOE: Moderate). With regard to the need for multiple insertion attempts, SGA was favored over ETI; for inadequate ventilation, ETI was favored over SGA (for both, SOE: Moderate). While these findings were based on a single study, it was an RCT with low ROB. ${ }^{17}$

Table 21. SGA versus ETI: additional outcomes

\begin{tabular}{|c|c|c|c|c|}
\hline Outcome & $\begin{array}{l}\text { Conclusion } \\
\text { (SOE) }\end{array}$ & $\begin{array}{l}\text { Number of } \\
\text { Studies }\end{array}$ & $\begin{array}{l}\text { Number of } \\
\text { Patients }\end{array}$ & Summary of Findings \\
\hline $\begin{array}{l}\text { Survival } \\
\text { 24-hour }\end{array}$ & $\begin{array}{l}\text { No conclusion } \\
\text { (Insufficient) }\end{array}$ & $3 \mathrm{OBS}^{55,65,68}$ & 29,777 & Inconsistent findings \\
\hline $\begin{array}{l}\text { Survival } \\
72 \text {-hour }\end{array}$ & $\begin{array}{l}\text { No difference } \\
\text { (Moderate) }\end{array}$ & $2 \mathrm{RCT}^{14,17,44}$ & 12,300 & $\begin{array}{l}\text { For one study, difference } \\
\text { favoring SGA in ITT and per- } \\
\text { protocol analyses, but no } \\
\text { difference in as-treated or } \\
\text { adjusted analyses. Second } \\
\text { study no difference. }\end{array}$ \\
\hline \multirow{2}{*}{$\begin{array}{l}\text { Survival } \\
\text { To ED, Hospital, } \\
\text { or ICU Admission }\end{array}$} & \multirow{2}{*}{$\begin{array}{l}\text { No difference } \\
\text { (Low) }\end{array}$} & $2 \mathrm{RCT}^{14,17,46}$ & 3,176 & No difference \\
\hline & & $5 \mathrm{OBS}^{25,26,32,49,62}$ & 17,506 & Inconsistent findings \\
\hline $\begin{array}{l}\text { Neurological } \\
\text { Function } \\
\text { GOS }\end{array}$ & $\begin{array}{l}\text { No conclusion } \\
\text { (Insufficient) }\end{array}$ & $1 \mathrm{OBS}^{30}$ & 78 & - \\
\hline $\begin{array}{l}\text { Oxygenation/ } \\
\text { Ventilation }\end{array}$ & $\begin{array}{l}\text { No difference } \\
\text { (Low) }\end{array}$ & $4 \mathrm{OBS}^{21,47,55,59}$ & 2,665 & $\begin{array}{l}\text { No difference ABG (1 study); } \\
\text { Sp02 (1 study); ETC02 ( } 3 \\
\text { studies) }\end{array}$ \\
\hline \multirow{2}{*}{$\begin{array}{l}\text { Harms: } \\
\text { Aspiration }\end{array}$} & \multirow{2}{*}{$\begin{array}{l}\text { No difference } \\
\text { (Moderate) }\end{array}$} & $1 \mathrm{RCT}^{44}$ & 9,296 & No difference \\
\hline & & $1 \mathrm{OBS}^{63}$ & 256 & No difference \\
\hline
\end{tabular}




\begin{tabular}{|c|c|c|c|c|}
\hline Outcome & $\begin{array}{l}\text { Conclusion } \\
\text { (SOE) }\end{array}$ & $\begin{array}{l}\text { Number of } \\
\text { Studies }\end{array}$ & $\begin{array}{l}\text { Number of } \\
\text { Patients }\end{array}$ & Summary of Findings \\
\hline \multirow{2}{*}{$\begin{array}{l}\text { Harms: } \\
\text { Oral/Airway } \\
\text { Trauma } \\
\end{array}$} & \multirow{2}{*}{$\begin{array}{l}\text { No difference } \\
\text { (Moderate) }\end{array}$} & $1 \mathrm{RCT}^{17}$ & 3,004 & No difference \\
\hline & & $1 \mathrm{OBS}^{18}$ & 155 & No difference \\
\hline $\begin{array}{l}\text { Harms: } \\
\text { Multiple ( } \geq 3) \\
\text { Insertions }\end{array}$ & $\begin{array}{l}\text { Favors SGA } \\
\text { (Moderate) }\end{array}$ & $1 \mathrm{RCT}^{17}$ & 3,004 & $\begin{array}{l}\text { Single study with low ROB } \\
\text { ETI } 1.3 \%, \text { SGA } 0.4 \% \\
\text { Difference }-0.9 ;(95 \% \mathrm{Cl}-1.7 \text { to } \\
-0.2) p=0.01\end{array}$ \\
\hline $\begin{array}{l}\text { Harms: } \\
\text { Inadequate Ventilation }\end{array}$ & $\begin{array}{l}\text { Favors ETI } \\
\text { (Moderate) }\end{array}$ & $1 \mathrm{RCT}^{17}$ & 3,004 & $\begin{array}{l}\text { Single study with low ROB } \\
\text { ETI } 0.6 \%, \text { SGA } 1.8 \% \\
\text { Difference } 1.2(95 \% \mathrm{Cl} 0.3 \text { to } \\
\text { 2.1) } p=0.01\end{array}$ \\
\hline $\begin{array}{l}\text { Harms: } \\
\text { Regurgitation }\end{array}$ & $\begin{array}{l}\text { No difference } \\
\text { (Moderate) }\end{array}$ & $1 \mathrm{RCT}^{44}$ & 9,296 & Single study with low ROB \\
\hline \multirow{2}{*}{$\begin{array}{l}\text { Harms: } \\
\text { Dislodged/Misplaced } \\
\text { Intubation } \\
\text { Any Complication } \\
\text { Fatal Complication } \\
\text { Need for Additional } \\
\text { Airway } \\
\text { Blood in Airway }\end{array}$} & \multirow[b]{2}{*}{$\begin{array}{l}\text { No conclusion } \\
\text { (Insufficient) }\end{array}$} & $2 \mathrm{RCT}^{17,44}$ & 12,300 & Inconsistent findings \\
\hline & & $4 \mathrm{OBS}^{18,49,54,69}$ & 1131 & $\begin{array}{l}\text { Inconsistent findings; single } \\
\text { OBS studies }\end{array}$ \\
\hline
\end{tabular}

$\mathrm{ABG}=$ arterial blood gas; $\mathrm{CI}=$ confidence interval; $\mathrm{ED}=$ emergency department; $\mathrm{ETI}=$ endotracheal intubation; $\mathrm{GOS}=$ Glasgow Outcome Scale; ICU = intensive care unit; ITT = intent to treat; NA = not applicable; OBS = observational study; RCT

$=$ randomized controlled trial; $\mathrm{ROB}=$ risk of bias; $\mathrm{RR}=$ risk ratio; $\mathrm{SGA}=$ supraglottic airway; $\mathrm{SOE}=$ strength of evidence

\section{Key Question 4: Modifiers Within Airway Approaches}

\section{Key Results}

\section{Key Results: Endotracheal Intubation}

In studies reporting on modifiers of ETI for prehospital airway management:

- ETI: Technique/device modifiers

- ETI with drug-facilitation versus without: rapid sequence intubation (RSI) versus no medication

- Survival to hospital discharge ( 6 studies; $\mathrm{N}=3,947$ ) was not significantly different for:

- Adult/mixed-age with trauma (Low SOE)

- First-pass success ( 8 studies; $\mathrm{N}=33,887$ ):

- Favored RSI for adult/mixed-age with mixed emergency types (Low SOE)

- Was not significantly different for adult/mixed-age with trauma (Low SOE)

- Overall success rate (6 studies; $\mathrm{N}=56,046$ ):

- Favored RSI for adults with trauma (Low SOE)

- Was not significantly different for:

- Adult cardiac arrest patients (Low SOE)

- Adults/mixed-age with mixed emergency types (Low SOE)

- Harms

- Occurred more often in the no medication group: recognized esophageal intubation and unrecognized mainstem intubation 
- No difference or inconsistent findings for other reported harms

○ ETI with drug-facilitation versus without: RSI versus sedation-facilitated

- First-pass success (3 studies; $\mathrm{N}=5,842$ ) favored RSI for:

- Adults/mixed-age with mixed emergency types (Low SOE)

- Harms

- No cases of esophageal intubation or aspiration observed

- ETI with drug-facilitation versus without: sedation-facilitated versus no medication

- First-pass success (3 studies; $N=19,262$ ) was not significantly different for:

- Adults/mixed-age with mixed emergency types (Low SOE)

- Video versus direct laryngoscopy

- First-pass success rate (12 studies; $N=7,280$ ) was not significantly different for:

- Adult cardiac arrest patients (Low SOE)

- Adult/mixed-age with mixed emergency types (Moderate SOE)

- Overall success rate (11 studies; $\mathrm{N}=3,060$ ) was not significantly different for:

- Adult cardiac arrest patients (Moderate SOE)

- Adult/mixed-age with mixed emergency types (Low SOE)

- Harms

- Problem with advancing the endotracheal tube reported more often with video laryngoscopy

- No difference for all other reported harms

- Laryngoscope blade material

- Favored metal blades for:

- First-pass success: disposable plastic versus reusable or disposable metal

- No difference for:

- First-pass success: reusable versus single-use metal blades

- All reported harms

○ Gum elastic bougie

- First-pass success and Definitive Airway Sans Hypoxia/Hypotension on First Attempt (DASH-1A): favored use of bougie

- No difference for overall success

- ETI: Patient characteristics modifiers

$\circ$ Emergency type

- First-pass success rate ( 9 studies; $\mathrm{N}=38,211$ )

- Favored medical for:

- Trauma versus medical: adult/mixed-age (Low SOE)

- No significant differences for:

○ Nonarrest versus cardiac arrest: adult/mixed-age (Low $\mathrm{SOE})$

- Overall success rate ( 9 studies; $\mathrm{N}=60,307$ ) was not significantly different for: 
- Medical versus cardiac arrest: adult/mixed-age (Low SOE)

- Trauma versus cardiac arrest: adult/mixed-age (Low SOE)

- Nonarrest versus cardiac arrest: adult/mixed-age (Low SOE)

- Trauma versus medical: adult/mixed-age (Low SOE)

- Age: age within pediatrics

- Poorer outcomes for infants versus toddlers or school-age children: survival, neurological function, first-pass success

- Better outcome for infants, versus toddlers or school-age children: aspiration pneumonia

- No differences for:

- Overall success across pediatric age groups

- Toddlers versus school-age children for any outcome

- Harms

- No differences across age groups within pediatrics for any reported harms

- Age: pediatric versus adult

- Favored adult for: overall success

- Was not different for: survival, DASH-1A

○ Age: elderly versus adult

- Was not different for: neurological function

- Sex: female versus male

- Favored female for: survival, ROSC

- Was not different for: overall success, DASH-1A

- Race: white versus nonwhite

- Poorer outcomes for nonwhite participants: ROSC, DASH-1A

\section{Key Results: Supraglottic Airway}

In studies reporting on modifiers of SGA for prehospital airway management:

- SGA: Technique/device modifiers

○ Perilaryngeal seal SGAs (i-gel vs. laryngeal mask airway [LMA]):

- Favored i-gel for: overall success

- Was not different for: survival, ROSC, first-pass success

- Pharyngeal versus perilaryngeal seal SGAs (laryngeal tube [LT], esophageal obturator, Combitube, or pharyngeotracheal lumen airway [PTLA] versus LMA)

- Was not different for: survival, neurological function, ventilation, arterial blood gases

- Harms

- Occurred more often with pharyngeal seal SGAs: inadequate ventilation, dislodgement, and total complications

- No difference for: tongue/pharyngeal swelling, bleeding, air leak, and incorrect placement

- Pharyngeal seal SGAs (Combitube versus PTLA):

- No difference: success, arterial blood gases

- Harms

- No difference for: inadequate ventilation

- SGA: Patient characteristics modifier 
- Age: pediatric versus adult

- Was not different for: first-pass success and overall success

There were 45 studies that compared outcomes across potential modifiers of ETI; 28 reported on outcomes which are analyzed qualitatively while 32 studies were pooled for one or more outcomes in meta-analysis. We identified eight studies reporting on potential modifiers of SGA, which were qualitatively analyzed. No studies about modifiers of BVM met inclusion criteria.

Results for Key Question 4 are organized first by airway intervention (ETI, SGA), then by modifier category (technique/device, patient characteristics). Results from meta-analyses and qualitative analyses are included, where applicable. Strength of evidence was not assessed for outcomes only analyzed qualitatively.

\section{Endotracheal Intubation}

\section{Endotracheal Intubation: Technique/Device}

There were four sets of comparisons for technique/device modifiers of ETI: ETI with drugfacilitation versus without; video versus direct laryngoscopy; laryngoscope blade material; and gum elastic bougie use.

There was enough data for pooled analysis of two sets of these comparisons: ETI with drugfacilitation versus without, and video versus direct laryngoscopy. Additional outcomes for these comparisons were analyzed qualitatively. We also qualitatively analyzed outcomes for comparisons of laryngoscope blade material and gum elastic bougie use.

\section{Technique/Device: Endotracheal Intubation With Drug Facilitation Versus Without}

To assess outcomes from variations in drug facilitation for ETI, we categorized interventions as RSI, sedation-facilitated, or no medication, resulting in three comparisons: RSI versus no medication; RSI versus sedation-facilitated; and sedation-facilitated versus no medication.

For first-pass success, meta-analysis was performed for all three comparisons. For survival and overall success outcomes, sufficient data for pooled analysis was only available for RSI versus no medication. For the other two comparisons, these outcomes are analyzed and summarized qualitatively. For all comparisons, additional outcomes and harms are also analyzed qualitatively.

\section{Rapid Sequence Intubation Versus No Medication}

We identified and analyzed 17 studies (16 observational studies and one CCT; $\mathrm{N}=165,621$ ) comparing RSI and ETI with no medication. ${ }^{61,67,70-84}$ Fourteen observational studies $(\mathrm{N}=144,206)$ provided data for pooled analysis of one or more outcomes. ${ }^{61,67,70,72-75,77-80,82-84}$

There were six studies $(\mathrm{N}=3,947)$ included for pooled analysis of survival to hospital discharge (Table 22, Appendix Figure H-12). Study populations were all adults or mixed-age; there were no studies in children. For patients with trauma in adult/mixed-age group, there was no difference in survival (SOE: Low). ${ }^{70,72,73,83}$ No changes in effects were detected in sensitivity analyses. For adults with cardiac arrest ${ }^{77}$ and adults with medical emergencies, ${ }^{75} \mathrm{SOE}$ was insufficient, as there was only one observational study for each subgroup. 
Table 22. Survival for RSI versus no medication as modifier of ETI by subgroup (no medication referent)

\begin{tabular}{|l|l|l|l|r|l|}
\hline $\begin{array}{l}\text { Emergency } \\
\text { Type }\end{array}$ & Age Group & $\begin{array}{l}\text { Conclusion } \\
\text { (SOE) }\end{array}$ & $\begin{array}{l}\text { Study Number } \\
\text { and Design }\end{array}$ & $\begin{array}{l}\text { Number of } \\
\text { Patients }\end{array}$ & $\begin{array}{l}\text { Summary of Results } \\
\text { RR (95\% CI), I }\end{array}$ \\
\hline $\begin{array}{l}\text { Cardiac } \\
\text { Arrest }\end{array}$ & Adults & $\begin{array}{l}\text { No conclusion } \\
\text { (Insufficient) }\end{array}$ & $1 \mathrm{OBS}^{77}$ & 3,047 & 3.69 (3.17 to 4.28), NA \\
\hline Trauma & $\begin{array}{l}\text { Adults/mixed- } \\
\text { age }\end{array}$ & $\begin{array}{l}\text { No difference } \\
\text { (Low) }\end{array}$ & $4 \mathrm{OBS}^{70,72,73,83}$ & 2,520 & 1.41 (0.83 to 2.46), $95.5 \%$ \\
\hline Medical & Adults & $\begin{array}{l}\text { No conclusion } \\
\text { (Insufficient) }\end{array}$ & $1 \mathrm{OBS}^{75}$ & 1,454 & 0.89 (0.82 to 0.97), NA \\
\hline
\end{tabular}

$\mathrm{CI}=$ confidence interval; ETI = endotracheal intubation; $\mathrm{I}^{2}=$ statistical test of heterogeneity; NA = not applicable; OBS = observational study; $\mathrm{RR}=$ risk ratio; $\mathrm{RSI}=$ rapid sequence intubation; $\mathrm{SOE}=$ strength of evidence

We included eight studies $(\mathrm{N}=33,887)$ for pooled analysis of first-pass success (Table 23, Appendix Figure H-13). ${ }^{61,74,77,79,80,82-84}$ Higher rates of first-pass success were observed with RSI in adults/mixed-age with mixed emergency types (SOE: Low). ${ }^{61,74,80,82,84}$ When high ROB studies were excluded from analysis, there was no difference for RSI compared to no medication, based on two remaining studies (Appendix Figure I-9). ${ }^{80,82}$ There was no difference among adults/mixed-age with trauma emergencies (SOE: Low). ${ }^{79,83}$ For adults with cardiac arrest ${ }^{77}$ and pediatrics with mixed emergency types, ${ }^{61} \mathrm{SOE}$ was insufficient, as there was only one observational study for each subgroup.

Table 23. First-pass success for RSI versus no medication as modifier of ETI by subgroup (no medication referent)

\begin{tabular}{|c|c|c|c|c|c|}
\hline $\begin{array}{l}\text { Emergency } \\
\text { Type }\end{array}$ & Age Group & $\begin{array}{l}\text { Conclusion } \\
\text { (SOE) }\end{array}$ & $\begin{array}{l}\text { Study Number } \\
\text { and Design }\end{array}$ & $\begin{array}{l}\text { Number of } \\
\text { Patients }\end{array}$ & $\begin{array}{l}\text { Summary of Results } \\
\text { RR }(95 \% \mathrm{Cl}), \mathrm{I}^{2}\end{array}$ \\
\hline $\begin{array}{l}\text { Cardiac } \\
\text { Arrest }\end{array}$ & Adults & $\begin{array}{l}\text { No conclusion } \\
\text { (Insufficient) }\end{array}$ & $1 \mathrm{OBS}^{77}$ & 2,776 & $0.89(0.82$ to 0.96$), \mathrm{NA}$ \\
\hline Trauma & $\begin{array}{l}\text { Adults/mixed- } \\
\text { age }\end{array}$ & $\begin{array}{l}\text { No difference } \\
\text { (Low) }\end{array}$ & $2 \mathrm{OBS}^{79,83}$ & 530 & 1.00 (0.88 to 1.14$), 11.9 \%$ \\
\hline \multirow{2}{*}{$\begin{array}{l}\text { Mixed } \\
\text { Emergency } \\
\text { Types }\end{array}$} & $\begin{array}{l}\text { Adults/mixed- } \\
\text { age }\end{array}$ & $\begin{array}{l}\text { Favors RSI } \\
\text { (Low) }\end{array}$ & 5 OBS $^{61,74,80,82,84}$ & 30,126 & 1.13 (1.09 to 1.17$), 62.2 \%$ \\
\hline & Pediatrics & $\begin{array}{l}\text { No conclusion } \\
\text { (Insufficient) }\end{array}$ & $1 \mathrm{OBS}^{61}$ & 455 & 1.37 (1.16 to 1.63$), \mathrm{NA}$ \\
\hline
\end{tabular}

$\mathrm{CI}=$ confidence interval; ETI $=$ endotracheal intubation; $\mathrm{I}^{2}=$ statistical test of heterogeneity; NA = not applicable; OBS = observational study; $\mathrm{RR}=$ risk ratio; $\mathrm{RSI}=$ rapid sequence intubation; $\mathrm{SOE}=$ strength of evidence

Six studies $(\mathrm{N}=56,046)$ were included for pooled analysis of overall success (Table 24, Appendix Figure H-13). ${ }^{67,70,77,78,80,84}$ All of the studies were in adults or mixed age group; none provided data separately for pediatric patients.

For adults with trauma emergencies, the two studies individually found higher overall success rates with RSI although the pooled results showed no difference. This is because the two studies differed in magnitude and precision of effect estimates, resulting in a wide confidence interval for the pooled result. The pooled confidence interval reflects study heterogeneity; thus our conclusion is that the comparison favors RSI (SOE: Low). Overall success rates were not different with RSI compared to no medication for three other subgroups: adults with cardiac arrest; ${ }^{67,77}$ adults with trauma; ${ }^{67,70}$ and adults/mixed age with mixed emergency types ${ }^{78,80,84}$ (for all, SOE: Low). For adults with medical emergencies, SOE was insufficient, as there was only one observational study. ${ }^{67}$ 
Table 24. Overall success for RSI versus no medication as modifier of ETI by subgroup (no medication referent)

\begin{tabular}{|c|c|c|c|c|c|}
\hline $\begin{array}{l}\text { Emergency } \\
\text { Type }\end{array}$ & Age Group & $\begin{array}{l}\text { Conclusion } \\
\text { (SOE) }\end{array}$ & $\begin{array}{l}\text { Study Number } \\
\text { and Design }\end{array}$ & $\begin{array}{l}\text { Number of } \\
\text { Patients }\end{array}$ & $\begin{array}{l}\text { Summary of Results } \\
\text { RR }(95 \% \mathrm{Cl}), \mathrm{I}^{2}\end{array}$ \\
\hline $\begin{array}{l}\text { Cardiac } \\
\text { Arrest }\end{array}$ & Adults & $\begin{array}{l}\text { No difference } \\
\text { (Low) }\end{array}$ & 2 OBS $^{67,77}$ & 30,496 & 1.04 (0.94 to 1.14$), 97.2 \%$ \\
\hline Trauma & Adults & $\begin{array}{l}\text { Favors RSIa } \\
\text { (Low) }\end{array}$ & $2 \mathrm{OBS}^{67,70}$ & 3,769 & 2.16 (0.59 to 8.40 ), $96.7 \%$ \\
\hline Medical & Adults & $\begin{array}{l}\text { No conclusion } \\
\text { (Insufficient) }\end{array}$ & $1 \mathrm{OBS}^{67}$ & 13,566 & 1.27 (1.25 to 1.29$), \mathrm{NA}$ \\
\hline $\begin{array}{l}\text { Mixed } \\
\text { Emergency } \\
\text { Types }\end{array}$ & $\begin{array}{l}\text { Adults/mixed- } \\
\text { age }\end{array}$ & $\begin{array}{l}\text { No difference } \\
\text { (Low) }\end{array}$ & $3 \mathrm{OBS}^{78,80,84}$ & 8,215 & 1.10 (0.87 to 1.36 ), $96.2 \%$ \\
\hline
\end{tabular}

$\mathrm{CI}=$ confidence interval; ETI = endotracheal intubation; $\mathrm{I}^{2}=$ statistical test of heterogeneity; NA = not applicable; OBS = observational study; $\mathrm{RR}=$ risk ratio; $\mathrm{RSI}=$ rapid sequence intubation; $\mathrm{SOE}=$ strength of evidence

${ }^{\text {a }}$ Pooled result shows no difference with wide CI due to study heterogeneity for effect estimates. Both studies favored RSI and our conclusion is that the comparison favors RSI.

Results from qualitative analysis of additional outcomes are summarized in Table 25. Length of stay in the ICU was reported in two studies. One study found that for survivors, the ICU stay was longer for the RSI group. ${ }^{70}$ However, the other study did not detect any difference for either ICU or hospital length of stay. ${ }^{75}$ All other outcomes were evaluated in only one study.

Table 25. Additional outcomes for RSI versus no medication as modifier of ETI

\begin{tabular}{|c|c|c|c|}
\hline Outcome & $\begin{array}{l}\text { Study Number } \\
\text { and Design }\end{array}$ & $\begin{array}{l}\text { Number of } \\
\text { Patients }\end{array}$ & Summary of Findings \\
\hline DASH-1A & $1 \mathrm{OBS}^{79}$ & 257 & Higher rates with RSI; OR 2.93 (95\% Cl 0.88 to 9.77$), p=0.08$ \\
\hline $\begin{array}{l}\text { Neurological } \\
\text { Function }\end{array}$ & $1 \mathrm{OBS}^{81}$ & 21 & $\begin{array}{l}\text { GOS scale of } 4 \text { or } 5 \text { at hospital discharge: } \\
\text { - } \quad \text { No difference }\end{array}$ \\
\hline $\begin{array}{l}\text { ICU Length of } \\
\text { Stay }\end{array}$ & $2 \mathrm{OBS}^{70,75}$ & 38,550 & $\begin{array}{l}\text { Inconsistent } \\
\text { - } \quad \text { Longer with RSI; mean days, AOR } 1.58(95 \% \mathrm{Cl} 1.30 \text { to } \\
\text { 1.92), p<0.000170 } \\
\text { - } \quad \text { No difference, median days }{ }^{75}\end{array}$ \\
\hline $\begin{array}{l}\text { Hospital } \\
\text { Length of Stay }\end{array}$ & $1 \mathrm{OBS}^{75}$ & 38,352 & No difference in mean length of stay \\
\hline $\begin{array}{l}\text { Oxygenation/ } \\
\text { Ventilation }\end{array}$ & $1 \mathrm{OBS}^{75}$ & 38,352 & $\begin{array}{l}\text { Larger increase in } \mathrm{SpO} 2 \text { in } \mathrm{RSI} \text { group } \\
\text { - } \mathrm{SpO}_{2} \text { difference, final prehospital minus initial: } 4.9 \text { vs. } \\
\text { 3.6; difference }-1.3(95 \% \mathrm{Cl}-2.5 \text { to }-0.06), p=0.04\end{array}$ \\
\hline
\end{tabular}

AOR $=$ adjusted odds ratio; $\mathrm{CI}=$ confidence interval; DASH-1A $=$ Definitive Airway Sans Hypoxia/Hypotension on First Attempt; ETI = endotracheal intubation; GOS = Glasgow Outcome Scale; ICU = intensive care unit; OR = odds ratio; OBS = observational study; RSI = rapid sequence intubation

Two studies provided data on comparative harms. Results for each harm are summarized by individual study (Table 26). The no medication group had higher rates of recognized esophageal intubation and unrecognized mainstem intubation. Hypotension was reported in two studies; one $^{72}$ found higher rates with no medication while the other study ${ }^{70}$ found no difference. There was no difference for RSI versus no medication for recognized mainstem intubation, hypoxia, or oral trauma. No cases of unrecognized esophageal intubation, tracheal perforation, or barotrauma were observed in either group. 
Table 26. Harms for RSI versus no medication as modifier of ETI

\begin{tabular}{|c|c|c|c|c|c|}
\hline $\begin{array}{l}\text { Harm } \\
\text { Description }\end{array}$ & $\begin{array}{l}\text { Study } \\
\text { Design }\end{array}$ & $\begin{array}{l}\text { Number } \\
\text { of } \\
\text { Patients } \\
\end{array}$ & $\begin{array}{l}\text { Proportion } \\
\text { Experienced } \\
\text { Harm: RSI } \\
\end{array}$ & $\begin{array}{l}\text { Proportion } \\
\text { Experienced Harm: } \\
\text { No Medication }\end{array}$ & Summary of Findings \\
\hline \multirow{2}{*}{$\begin{array}{l}\text { Esophageal } \\
\text { Intubation }\end{array}$} & \multirow{2}{*}{$\mathrm{OBS}^{83}$} & \multirow{2}{*}{283} & $0 \%(0 / 140)$ & $0 \%(0 / 143)$ & $\begin{array}{l}\text { Unrecognized: none observed } \\
\text { in either group }\end{array}$ \\
\hline & & & $4 \%(5 / 140)$ & $20 \%(28 / 143)$ & $\begin{array}{l}\text { Recognized: occurred more } \\
\text { often in no medication group }\end{array}$ \\
\hline \multirow{2}{*}{$\begin{array}{l}\text { Mainstem } \\
\text { Intubation }\end{array}$} & \multirow[t]{2}{*}{$\mathrm{OBS}^{83}$} & \multirow[t]{2}{*}{283} & $0 \%(0 / 140)$ & $8 \%(11 / 143)$ & $\begin{array}{l}\text { Unrecognized: occurred more } \\
\text { often in no medication group }\end{array}$ \\
\hline & & & $1 \%(1 / 140)$ & $1 \%(2 / 143)$ & Recognized: no difference \\
\hline \multirow{3}{*}{ Hypotension $^{a}$} & \multirow{2}{*}{$\mathrm{OBS}^{70}$} & \multirow{2}{*}{272} & $15.4 \%(27 / 175)$ & $11.7 \%(10 / 85)$ & No difference; prehospital \\
\hline & & & $11.7 \%(20 / 171)$ & $9.0 \%(8 / 89)$ & No difference; on ED arrival \\
\hline & $\mathrm{OBS}^{72}$ & 1,077 & $23 \%(117 / 774)$ & $37 \%(76 / 303)$ & $\begin{array}{l}\text { Occurred more often in no } \\
\text { medication group, } p<0.001\end{array}$ \\
\hline \multirow{2}{*}{ Hypoxiab $^{b}$} & \multirow{2}{*}{$\mathrm{OBS}^{70}$} & \multirow{2}{*}{283} & $14.6 \%(28 / 192)$ & $17.5 \%(16 / 91)$ & No difference; prehospital \\
\hline & & & $11.2 \%(21 / 187)$ & $8.2 \%(7 / 85)$ & No difference; on ED arrival \\
\hline Oral Trauma & $\mathrm{OBS}^{83}$ & 283 & $0 \%(0 / 140)$ & $1 \%(2 / 143)$ & No difference \\
\hline $\begin{array}{l}\text { Tracheal } \\
\text { Perforation }\end{array}$ & $\mathrm{OBS}^{83}$ & 283 & $0 \%(0 / 140)$ & $0 \%(0 / 143)$ & None observed in either group \\
\hline Barotrauma & $\mathrm{OBS}^{83}$ & 283 & $0 \%(0 / 140)$ & $0 \%(0 / 143)$ & None observed in either group \\
\hline
\end{tabular}

$\mathrm{ED}=$ emergency department; ETI = endotracheal intubation; OBS = observational study; RSI = rapid sequence intubation

${ }^{\text {a }}$ Hypotension $=\mathrm{SBP}<90 \mathrm{mmHg}$

${ }^{\mathrm{b}}$ Hypoxia $=\mathrm{SaO}_{2}<90 \%$

\section{Rapid Sequence Intubation Versus Sedation-Facilitated}

Five studies (4 observational and $1 \mathrm{CCT} ; \mathrm{N}=31,778$ ) were included for the comparison of RSI and ETI with sedation facilitation. ${ }^{61,71,74,81,84}$

Three observational studies $(\mathrm{N}=5,842)$ provided data for pooled analysis of first-pass success (Table 27, Appendix Figure H-14) ${ }^{61,74,84}$ For adult/mixed age patients with mixed emergency types, there were higher rates of first-pass success with RSI (SOE: Low) ${ }^{61,74,84}$ SOE was insufficient for children with mixed emergency types, as there was only one observational study. ${ }^{61}$

Table 27. First-pass success for RSI versus sedation-facilitated as modifier of ETI by subgroup (sedation-facilitated referent)

\begin{tabular}{|l|l|l|l|l|l|}
\hline $\begin{array}{l}\text { Emergency } \\
\text { Type }\end{array}$ & Age Group & $\begin{array}{l}\text { Conclusion } \\
\text { (SOE) }\end{array}$ & $\begin{array}{l}\text { Study Number } \\
\text { and Design }\end{array}$ & $\begin{array}{l}\text { Number of } \\
\text { Patients }\end{array}$ & $\begin{array}{l}\text { Summary of Results } \\
\text { RR (95\% Cl), } \mathbf{2}^{\mathbf{2}}\end{array}$ \\
\hline $\begin{array}{l}\text { Mixed } \\
\text { Emergency } \\
\text { Types }\end{array}$ & $\begin{array}{l}\text { Adults/mixed- } \\
\text { age }\end{array}$ & $\begin{array}{l}\text { Favors RSI } \\
\text { (Low) }\end{array}$ & 3 OBS ${ }^{61,74,84}$ & 5,778 & 1.11 (1.05 to 1.18), 0.0\% \\
\cline { 2 - 6 } & Pediatrics & $\begin{array}{l}\text { No conclusion } \\
\text { (Insufficient) }\end{array}$ & 1 OBS ${ }^{61}$ & 64 & 1.45 (0.88 to 2.39), NA \\
\hline
\end{tabular}

$\mathrm{CI}=$ confidence interval; ETI $=$ endotracheal intubation; $\mathrm{I}^{2}=$ statistical test of heterogeneity; NA = not applicable; OBS = observational study; $\mathrm{RSI}=$ rapid sequence intubation; $\mathrm{SOE}=$ strength of evidence

Additional outcomes and harms were qualitatively analyzed and are summarized in Tables 28 and 29, respectively. Overall success rates were higher with RSI compared to sedation-facilitated ETI. ${ }^{71,84}$ There was no statistically significant difference between groups for neurological function at hospital discharge. ${ }^{81}$ There was limited reporting of harms in the included studies, with only one study providing comparative data. ${ }^{71}$ In that study, no cases of esophageal intubation or aspiration were observed in either group. 
Table 28. Additional outcomes for RSI versus sedation-facilitated as modifier of ETI

\begin{tabular}{|l|l|r|l|}
\hline Outcome & $\begin{array}{l}\text { Study Number } \\
\text { and Design }\end{array}$ & $\begin{array}{l}\text { Number of } \\
\text { Patients }\end{array}$ & \begin{tabular}{l} 
Summary of Findings \\
\hline $\begin{array}{l}\text { Overall } \\
\text { Success }\end{array}$
\end{tabular} \\
$1 \mathrm{CCT}^{71}, 1 \mathrm{OBS}^{84}$ & 255 & $\begin{array}{r}\text { Higher rates with RSI } \\
\bullet \quad 96 \% \text { vs. } 71 \%, \mathrm{p}<0.03^{71}\end{array}$ \\
\hline $\begin{array}{l}\text { Neurological } \\
\text { Function }\end{array}$ & $1 \mathrm{OBS}^{81}$ & 68 & $\begin{array}{l}\text { GOS scale of } 4 \text { or } 5 \text { at hospital discharge: } \\
\text { No difference }\end{array}$ \\
\hline
\end{tabular}

$\mathrm{CCT}=$ controlled clinical trial; ETI = endotracheal intubation; GOS = Glasgow Outcome Scale; OBS = observational study; RSI

$=$ rapid sequence intubation

Table 29. Harms for RSI versus sedation-facilitated as modifier of ETI

\begin{tabular}{|l|l|l|l|l|l|}
\hline $\begin{array}{l}\text { Harm } \\
\text { Description }\end{array}$ & $\begin{array}{l}\text { Study } \\
\text { Design }\end{array}$ & $\begin{array}{l}\text { Number } \\
\text { of } \\
\text { Patients }\end{array}$ & $\begin{array}{l}\text { Proportion } \\
\text { Experienced } \\
\text { Harm: RSI }\end{array}$ & $\begin{array}{l}\text { Proportion } \\
\text { Experienced Harm: } \\
\text { Sedation-Facilitated }\end{array}$ & Summary of Findings \\
\hline $\begin{array}{l}\text { Esophageal } \\
\text { Intubation }\end{array}$ & $\mathrm{CCT}^{71}$ & 49 & $0 \%(0 / 25)$ & $0 \%(0 / 24)$ & $\begin{array}{l}\text { No cases observed for either } \\
\text { group; not specified if } \\
\text { recognized or unrecognized }\end{array}$ \\
\hline Aspiration & $\mathrm{CCT}^{71}$ & 49 & $0 \%(0 / 25)$ & $0 \%(0 / 24)$ & $\begin{array}{l}\text { No cases observed for either } \\
\text { group }\end{array}$ \\
\hline
\end{tabular}

$\mathrm{CCT}=$ controlled clinical trial; ETI $=$ endotracheal intubation; $\mathrm{RSI}=$ rapid sequence intubation

\section{Sedation-Facilitated Versus No Medication}

Four observational studies $(\mathrm{N}=19,325)$ were included for comparison of ETI with sedation facilitation versus ETI with no medication. ${ }^{61,74,81,84}$

Three studies $(\mathrm{N}=19,262)$ provided data for pooled analysis of first-pass success (Table 30, Appendix Figure H-15). ${ }^{61,74,84}$ There was no difference for adults/mixed-age with mixed emergency types (SOE: Low). ${ }^{61,74,84}$ For children with mixed emergency types, SOE was insufficient, as there was only one observational study. ${ }^{61}$

Additional outcomes were qualitatively analyzed and are summarized in Table 31 . There were better outcomes with sedation-facilitated ETI for neurological function. ${ }^{81}$ Overall success rates were similar between groups. ${ }^{84}$

None of the included studies reported on comparative harms outcomes for sedationfacilitated ETI versus ETI without medication.

Table 30. First-pass success for sedation-facilitated versus no medication as modifier of ETI by subgroup (no medication referent)

\begin{tabular}{|l|l|l|l|r|l|}
\hline $\begin{array}{l}\text { Emergency } \\
\text { Type }\end{array}$ & Age Group & $\begin{array}{l}\text { Conclusion } \\
\text { (SOE) }\end{array}$ & $\begin{array}{l}\text { Study Number } \\
\text { and Design }\end{array}$ & $\begin{array}{l}\text { Number of } \\
\text { Patients }\end{array}$ & $\begin{array}{l}\text { Summary of Results } \\
\text { RR (95\% Cl), } \mathbf{I}^{\mathbf{2}}\end{array}$ \\
\hline $\begin{array}{l}\text { Mixed } \\
\text { Emergency } \\
\text { Types }\end{array}$ & Adults/mixed-age & $\begin{array}{l}\text { No difference } \\
\text { (Low) }\end{array}$ & 3 OBS $^{61,74,84}$ & 18,841 & $1.02(0.77$ to 1.27$), 0.0 \%$ \\
\cline { 2 - 6 } & Pediatrics & $\begin{array}{l}\text { No conclusion } \\
\text { (Insufficient) }\end{array}$ & 1 OBS $^{61}$ & 421 & 0.95 (0.58 to 1.53), NA \\
\hline
\end{tabular}

$\mathrm{CI}=$ confidence interval; ETI $=$ endotracheal intubation; $\mathrm{I}^{2}=$ statistical test of heterogeneity; NA = not applicable; OBS = observational study; $\mathrm{RR}=$ risk ratio; $\mathrm{SOE}=$ strength of evidence

Table 31. Additional outcomes for sedation-facilitated versus no medication as modifier of ETI

\begin{tabular}{|c|c|c|c|}
\hline Outcome & $\begin{array}{l}\text { Study Number } \\
\text { and Design }\end{array}$ & $\begin{array}{l}\text { Number of } \\
\text { Patients }\end{array}$ & Summary of Findings \\
\hline $\begin{array}{l}\text { Overall } \\
\text { Success }\end{array}$ & $1 \mathrm{OBS}^{84}$ & 589 & No difference \\
\hline $\begin{array}{l}\text { Neurological } \\
\text { Function }\end{array}$ & $1 \mathrm{OBS}^{81}$ & 63 & $\begin{array}{l}\text { GOS scale of } 4 \text { or } 5 \text { at hospital discharge: } \\
\text { - Higher proportion with sedation-facilitated; } 62 \% \text { vs. } \\
13 \%, p=0.0176\end{array}$ \\
\hline
\end{tabular}

ETI = endotracheal intubation; GOS = Glasgow Outcome Scale; OBS = observational study 


\section{Technique/Device: Video Versus Direct Laryngoscopy}

We identified and analyzed 15 studies (6 RCTs, 9 observational; $\mathrm{N}=8,968$ ) that compared outcomes of first-pass success and overall success for video and direct laryngoscopy. ${ }^{51,74,79,85-96}$ Fourteen studies enrolled adults only, and one study ${ }^{90}$ included patients of all ages (mixed-age).

Twelve studies were pooled to obtain estimates for first-pass success (Table 32, Appendix Figure H-16). ${ }^{51,74,79,85-93}$ There was no difference in rates of first-pass success for video versus direct laryngoscopy in RCTs and observational studies of adult patients with cardiac arrest (SOE: Low). ${ }^{51,85,87,88,93}$ There was also no difference for video versus direct laryngoscopy in RCTs and observational studies of adults or mixed-age with mixed emergency types (SOE: Moderate). ${ }^{86,88-}$ ${ }^{92}$ SOE was insufficient for adults with medical emergencies and adults with trauma emergencies. No changes in effects were detected in sensitivity analyses.

Table 32. First-pass success for video versus direct laryngoscopy as modifier of ETI by subgroup (direct laryngoscopy referent)

\begin{tabular}{|c|c|c|c|c|c|}
\hline $\begin{array}{l}\text { Emergency } \\
\text { Type }\end{array}$ & Age Group & $\begin{array}{l}\text { Conclusion } \\
\text { (SOE) }\end{array}$ & $\begin{array}{l}\text { Study Number } \\
\text { and Design }\end{array}$ & $\begin{array}{l}\text { Number of } \\
\text { Patients }\end{array}$ & $\begin{array}{l}\text { Summary of Results } \\
\text { RR }(95 \% \mathrm{Cl}), \mathrm{I}^{2}\end{array}$ \\
\hline \multirow{2}{*}{$\begin{array}{l}\text { Cardiac } \\
\text { Arrest }\end{array}$} & \multirow{2}{*}{ Adults } & \multirow{2}{*}{$\begin{array}{l}\text { No difference } \\
\text { (Low) }\end{array}$} & $2 \mathrm{RCT}^{85,87}$ & 191 & 0.76 (0.46 to 1.26$), 69.8 \%$ \\
\hline & & & 3 OBS $^{51,88,93}$ & 714 & 1.19 (0.74 to 1.87$), 87.6 \%$ \\
\hline \multirow{2}{*}{ Trauma } & \multirow{2}{*}{ Adults } & \multirow{2}{*}{$\begin{array}{l}\text { No conclusion } \\
\text { (Insufficient) }\end{array}$} & No RCT & - & - \\
\hline & & & $2 \mathrm{OBS}^{74,79}$ & 310 & 1.01 (0.30 to 1.93 ), $74.9 \%$ \\
\hline \multirow{2}{*}{ Medical } & \multirow{2}{*}{ Adults } & \multirow{2}{*}{$\begin{array}{l}\text { No conclusion } \\
\text { (Insufficient) }\end{array}$} & No RCT & - & - \\
\hline & & & $1 \mathrm{OBS}^{74}$ & 249 & 1.18 (1.02 to 1.36$), \mathrm{NA}$ \\
\hline \multirow{2}{*}{$\begin{array}{l}\text { Mixed } \\
\text { Emergency }\end{array}$} & \multirow{2}{*}{$\begin{array}{l}\text { Adults/mixed- } \\
\text { age }\end{array}$} & \multirow{2}{*}{$\begin{array}{l}\text { No difference } \\
\text { (Moderate) }\end{array}$} & $2 \mathrm{RCT}^{89,91}$ & 666 & 1.06 (0.81 to 1.41$), 88.7 \%$ \\
\hline & & & $4 \mathrm{OBS}^{86,88,90,92}$ & 5,150 & 1.22 (0.96 to 1.63 ), $85.8 \%$ \\
\hline
\end{tabular}

$\mathrm{CI}=$ confidence interval; ETI $=$ endotracheal intubation; $\mathrm{I}^{2}=$ statistical test of heterogeneity; NA = not applicable; OBS = observational study; $\mathrm{RCT}=$ randomized control trial; $\mathrm{RR}=$ risk ratio; $\mathrm{SOE}=$ strength of evidence

Eleven studies were pooled to obtain estimates for overall success (Table 33, Appendix Figure H-16). ${ }^{51,85-90,93-96}$ There was no difference in rates of overall success for video versus direct laryngoscopy in RCTs and observational studies of adult patients with cardiac arrest (SOE: Moderate). ${ }^{51,85,87,88,93}$ For adults or mixed-age with mixed emergency types there was no difference in RCTs and observational studies (SOE: Low). ${ }^{86,88-90,94-96}$ No changes in effect were detected in sensitivity analyses for either subgroup.

In qualitative analysis, there was no difference in video versus direct laryngoscopy for DASH-1 $\mathrm{A}^{79}$ or ROSC ${ }^{51,86}$ in observational studies (Table 34 ).

Table 33. Overall success for video versus direct laryngoscopy as modifier of ETI by subgroup (direct laryngoscopy referent)

\begin{tabular}{|c|c|c|c|c|c|}
\hline $\begin{array}{l}\text { Emergency } \\
\text { Type }\end{array}$ & Age Group & $\begin{array}{l}\text { Conclusion } \\
\text { (SOE) }\end{array}$ & $\begin{array}{l}\text { Study Number } \\
\text { and Design }\end{array}$ & $\begin{array}{l}\text { Number of } \\
\text { Patients }\end{array}$ & $\begin{array}{l}\text { Summary of Results } \\
\text { RR }(95 \% \mathrm{Cl}), \mathrm{I}^{2}\end{array}$ \\
\hline \multirow{2}{*}{$\begin{array}{l}\text { Cardiac } \\
\text { Arrest }\end{array}$} & \multirow{2}{*}{ Adults } & \multirow{2}{*}{$\begin{array}{l}\text { No difference } \\
\text { (Moderate) }\end{array}$} & $2 \mathrm{RCT}^{85,87}$ & 191 & 0.96 (0.86 to 1.04 ), $0 \%$ \\
\hline & & & $3 \mathrm{OBS}^{51,8893}$ & 714 & 1.19 (0.94 to 1.49$), 78.5 \%$ \\
\hline \multirow{2}{*}{$\begin{array}{l}\text { Mixed } \\
\text { Emergency }\end{array}$} & \multirow{2}{*}{$\begin{array}{l}\text { Adults/mixed- } \\
\text { age }\end{array}$} & \multirow{2}{*}{$\begin{array}{l}\text { No difference } \\
\text { (Low) }\end{array}$} & $3 \mathrm{RCT}^{89,94,95}$ & 1,053 & 0.68 (0.42 to 1.09 ), $97.6 \%$ \\
\hline & & & $4 \mathrm{OBS}^{86,88,90,96}$ & 1,102 & 1.15 (0.98 to 1.40$) ; 85.7 \%$ \\
\hline
\end{tabular}

$\mathrm{CI}=$ confidence interval; $\mathrm{ETI}=$ endotracheal intubation; $\mathrm{I}^{2}=$ statistical test of heterogeneity; OBS = observational study; RCT = randomized control trial; $\mathrm{SOE}=$ strength of evidence 
Table 34. Additional outcomes for video versus direct laryngoscopy as modifier of ETI

\begin{tabular}{|l|l|r|l|}
\hline Outcome & $\begin{array}{l}\text { Study Number } \\
\text { and Design }\end{array}$ & $\begin{array}{l}\text { Number of } \\
\text { Patients }\end{array}$ & Summary of Findings \\
\hline DASH-1A & 1 OBS $^{79}$ & 256 & No difference \\
\hline ROSC & 2 OBS $^{51,86}$ & 489 & $\begin{array}{r}\text { No difference } \\
\text { Prehospital ROSC } \\
\text { - ROSC timing not specified }\end{array}$ \\
\hline
\end{tabular}

ETI = endotracheal intubation; DASH-1A = Definitive Airway Sans Hypoxia/Hypotension on First Attempt; OBS = observational study; ROSC $=$ return of spontaneous circulation

Five studies provided data for qualitative analysis of comparative harms for video and direct laryngoscopy. ${ }^{74,86,89,95,96}$ Results for each harm are summarized by individual study in Table 35 . Similar proportions of patients experienced reported harms in both groups (peri-intubation cardiac arrest, esophageal intubations, oral or airway trauma, regurgitation, and total complications).

Table 35. Harms for video versus direct laryngoscopy as modifier of ETI

\begin{tabular}{|c|c|c|c|c|c|}
\hline $\begin{array}{l}\text { Harm } \\
\text { Description }\end{array}$ & $\begin{array}{l}\text { Study } \\
\text { Design }\end{array}$ & $\begin{array}{l}\text { Number of } \\
\text { Patients }\end{array}$ & $\begin{array}{l}\text { Proportion } \\
\text { Experienced } \\
\text { Harm: Video }\end{array}$ & $\begin{array}{l}\text { Proportion } \\
\text { Experienced } \\
\text { Harm: Direct }\end{array}$ & Summary of Findings \\
\hline $\begin{array}{l}\text { Peri-Intubation } \\
\text { Cardiac Arrest }\end{array}$ & $\mathrm{OBS}^{74}$ & 296 & $2.8 \%(5 / 181)$ & $2.6 \%(3 / 151)$ & No difference \\
\hline \multirow{3}{*}{$\begin{array}{l}\text { Esophageal } \\
\text { Intubation }\end{array}$} & $\mathrm{RCT}^{95}$ & 326 & $1.8 \%(3 / 168)$ & $2.5 \%(4 / 158)$ & $\begin{array}{l}\text { No difference; not specified if } \\
\text { recognized or unrecognized }\end{array}$ \\
\hline & $\mathrm{RCT}^{89}$ & 579 & $4.8 \%(14 / 294)$ & $3.2 \%(9 / 285)$ & $\begin{array}{l}\text { No difference; not specified if } \\
\text { recognized or unrecognized }\end{array}$ \\
\hline & $\mathrm{OBS}^{96}$ & 615 & $0 \%(0 / 300)$ & $0 \%(0 / 315)$ & $\begin{array}{l}\text { None occurred in either group; } \\
\text { unrecognized esophageal } \\
\text { intubations }\end{array}$ \\
\hline \multirow{2}{*}{$\begin{array}{l}\text { Oral/Airway } \\
\text { Trauma }\end{array}$} & $\mathrm{RCT}^{89}$ & 579 & $2.7 \%(8 / 294)$ & $2.1 \%(6 / 285)$ & No difference \\
\hline & OBS $^{96}$ & 615 & $0.6 \%(2 / 315)$ & $1.0 \%(3 / 300)$ & No difference \\
\hline Regurgitation & OBS $^{96}$ & 615 & $4.8 \%(15 / 315)$ & $4.3 \%(13 / 300)$ & No difference \\
\hline \multirow{2}{*}{$\begin{array}{l}\text { Problems } \\
\text { Advancing the } \\
\text { Endotracheal } \\
\text { Tube }\end{array}$} & \multirow{2}{*}{$\mathrm{RCT}^{95}$} & \multirow{2}{*}{326} & $18.4 \%(31 / 168)$ & $1.9 \%(3 / 158)$ & $\begin{array}{l}\text { To larynx: higher rate with } \\
\text { video laryngoscopy, } p<0.0001\end{array}$ \\
\hline & & & $9.5 \%(16 / 168)$ & $1.9 \%(3 / 158)$ & $\begin{array}{l}\text { To trachea: higher rate with } \\
\text { video laryngoscopy, } p=0.0036\end{array}$ \\
\hline Complications & $\mathrm{OBS}^{86}$ & 212 & $1 \%(1 / 89)$ & $2 \%(3 / 123)$ & No difference \\
\hline
\end{tabular}

$\mathrm{ETI}=$ endotracheal intubation; OBS $=$ observational study; $\mathrm{RCT}=$ randomized control trial

\section{Technique/Device: Laryngoscope Blades and Gum Elastic Bougie}

Additional technique/device comparisons for ETI included laryngoscope blade material (1 RCT, ${ }^{97} 2$ observational studies; ${ }^{98,99} \mathrm{~N}=4,466$ ) and gum elastic bougie use ( 2 observational studies, ${ }^{78,79} \mathrm{~N}=410$ ). Results for outcomes were analyzed qualitatively and these are summarized in Table 36. Reusable metal blades were associated with higher rates of first-pass success compared with plastic disposable blades, ${ }^{98,99}$ and similar rates versus single-use metal blades. ${ }^{97}$ Use of gum elastic bougie was associated with successful DASH-1A and first-pass success in a study involving helicopter EMS providers (flight registered nurse, nurse practitioner, or emergency physician). ${ }^{79}$ For overall success, there was no difference in rates with bougie use versus without, in a study with paramedic and emergency medical technician providers. ${ }^{78}$

Two studies provided data on harms outcomes for comparisons of laryngoscope blade material. ${ }^{97,98}$ Results for each harm are summarized by individual study in Table 37 . Across 
reported harms, there was no difference in proportions of patients experiencing harm for both metal versus plastic blades and for reusable versus disposable metal blades.

No eligible studies reported on comparative harms for gum elastic bougie use.

Table 36. Outcomes for comparisons of laryngoscope blades and use of gum elastic bougie as modifiers of ETI

\begin{tabular}{|c|c|c|c|c|}
\hline Comparison & Outcome & $\begin{array}{l}\text { Study Number } \\
\text { and Design }\end{array}$ & $\begin{array}{l}\text { Number of } \\
\text { Patients }\end{array}$ & Summary of Findings \\
\hline $\begin{array}{l}\text { Reusable vs. } \\
\text { Single-Use } \\
\text { Metal Blades }\end{array}$ & $\begin{array}{l}\text { First-pass } \\
\text { success }\end{array}$ & $1 \mathrm{RCT}^{97}$ & 817 & No difference \\
\hline $\begin{array}{l}\text { Metal vs. } \\
\text { Plastic Blades }\end{array}$ & $\begin{array}{l}\text { First-pass } \\
\text { success }\end{array}$ & $2 \mathrm{OBS}^{98,99}$ & 3,649 & $\begin{array}{l}\text { Higher rates of first-pass success with } \\
\text { metal blades than plastic blades } \\
\text { - } \quad \text { Reusable metal vs. disposable } \\
\text { plastic: OR } 1.94 \text { (95\% Cl } 1.17 \text { to } \\
\text { 3.41), p<0.00199 } \\
\text { Disposable metal vs. disposable } \\
\text { plastic: } 84 \% \text { vs. } 76 \%, p<0.002^{98}\end{array}$ \\
\hline \multirow{3}{*}{$\begin{array}{l}\text { Gum Elastic } \\
\text { Bougie }\end{array}$} & DASH-1A & $1 \mathrm{OBS}^{79}$ & 263 & $\begin{array}{l}\text { Use of gum elastic bougie associated with } \\
\text { successful DASH-1A; AOR } 5.38(95 \% \mathrm{Cl} \\
1.83 \text { to } 15.8), p=0.002\end{array}$ \\
\hline & $\begin{array}{l}\text { First-pass } \\
\text { success }\end{array}$ & $1 \mathrm{OBS}^{79}$ & 263 & $\begin{array}{l}\text { Use of gum elastic bougie associated with } \\
\text { first-pass success; AOR } 7.79(95 \% \mathrm{Cl} 2.31 \\
\text { to } 26.3), p<0.001\end{array}$ \\
\hline & Overall success & $1 \mathrm{OBS}^{78}$ & 147 & No difference \\
\hline
\end{tabular}

AOR = adjusted odds ratio; $\mathrm{CI}=$ confidence interval; ETI = endotracheal intubation; DASH-1A = Definitive Airway Sans Hypoxia/Hypotension on First Attempt; OBS = observational study; OR = odds ratio; RCT = randomized control trial

Table 37. Harms for comparisons of laryngoscope blades as modifier of ETI

\begin{tabular}{|l|l|l|l|l|l|}
\hline & Study \\
Harm Description & $\begin{array}{l}\text { Number of } \\
\text { Patients }\end{array}$ & $\begin{array}{l}\text { Proportion } \\
\text { Experienced } \\
\text { Harm for: }\end{array}$ & $\begin{array}{l}\text { Versus } \\
\text { Proportion } \\
\text { Experienced } \\
\text { Harm for: }\end{array}$ & Summary of Findings \\
\hline $\begin{array}{l}\text { Unrecognized } \\
\text { Intubation }\end{array}$ & OBS & 2,472 & $\begin{array}{l}\text { Metal blades: } \\
0 \%(0 / 1,395)\end{array}$ & $\begin{array}{l}\text { Plastic blades: } \\
0 \%(0 / 1,077)\end{array}$ & None occurred in either group \\
\hline Hypotension & RCT $^{97}$ & 817 & $\begin{array}{l}\text { Reusable } \\
\text { metal blades: } \\
7 \%(29 / 408)\end{array}$ & $\begin{array}{l}\text { Disposable } \\
\text { metal blades: } \\
10 \%(39 / 409)\end{array}$ & No difference \\
\hline Dental Trauma & RCT $^{97}$ & 817 & $\begin{array}{l}\text { Reusable } \\
\text { metal blades: } \\
0.2 \%(1 / 408)\end{array}$ & $\begin{array}{l}\text { Disposable } \\
\text { metal blades: } \\
0.2 \%(1 / 409)\end{array}$ & No difference \\
\hline Vomiting & $\mathrm{RCT}^{97}$ & 817 & $\begin{array}{l}\text { Reusable } \\
\text { metal blades: } \\
2 \%(6 / 408)\end{array}$ & $\begin{array}{l}\text { Disposable } \\
\text { metal blades: } \\
2 \%(7 / 409)\end{array}$ & No difference \\
\hline Any Complication & RCT ${ }^{97}$ & 817 & $\begin{array}{l}\text { Reusable } \\
\text { metal blades: } \\
19 \%(76 / 408)\end{array}$ & $\begin{array}{l}\text { Disposable } \\
\text { metal blades: } \\
21 \%(87 / 409)\end{array}$ & No difference \\
\hline
\end{tabular}

ETI = endotracheal intubation; OBS = observational study; RCT = randomized control trial

\section{Endotracheal Intubation: Patient Characteristics}

The included studies reported on several modifiers of ETI related to patient characteristics. Pooled analysis was possible only for comparisons of emergency type, while comparisons of age, sex, and race were summarized qualitatively. 


\section{Patient Characteristics: Emergency Type}

Ten studies $(\mathrm{N}=101,799)$ provided data for pooled analysis of emergency type comparisons for first-pass success or overall success outcomes (Tables 38 and 39, Appendix Figures 17 to 20). ${ }^{61,67,74,80,84,100-104}$ No eligible studies provided data on comparative harms for emergency types.

First-pass success rates were higher in medical emergencies than trauma emergencies among adults/mixed age patients (SOE: Low) ${ }^{61,74,101,104}$ In adults/mixed age patients, rates were similar for nonarrest versus cardiac arrest emergencies (SOE: Low) ${ }^{80,84,88,100,103}$ SOE was insufficient for first-pass success rates in adults/mixed age patients for medical versus cardiac arrest and for trauma versus cardiac arrest, due to study limitations and inconsistent findings. In pediatric patients, SOE was insufficient for all emergency type comparisons, as there was only one observational study. ${ }^{61}$

There was no difference in overall success rates for all comparisons of emergency types in studies of adults/mixed-age (for all, SOE: Low).

Table 38. First-pass success for comparisons of emergency type as modifier of ETI

\begin{tabular}{|c|c|c|c|c|c|}
\hline Comparison & Subgroup & $\begin{array}{l}\text { Conclusion } \\
\text { (SOE) }\end{array}$ & $\begin{array}{l}\text { Study Number } \\
\text { and Design }\end{array}$ & $\begin{array}{l}\text { Number of } \\
\text { Patients }\end{array}$ & $\begin{array}{l}\text { Summary of Results } \\
\text { RR }(95 \% \mathrm{Cl}), \mathrm{I}^{2}\end{array}$ \\
\hline \multirow{2}{*}{$\begin{array}{l}\text { Medical vs. } \\
\text { Cardiac } \\
\text { Arrest }^{\mathrm{a}}\end{array}$} & $\begin{array}{l}\text { Adults/mixed- } \\
\text { age }\end{array}$ & $\begin{array}{l}\text { No conclusion } \\
\text { (Insufficient) }\end{array}$ & $2 \mathrm{OBS}^{61,104}$ & 20,644 & $0.90(0.61$ to 1.26$), 0 \%$ \\
\hline & Pediatrics & $\begin{array}{l}\text { No conclusion } \\
\text { (Insufficient) }\end{array}$ & $1 \mathrm{OBS}^{61}$ & 409 & 0.97 (0.80 to 1.19$), \mathrm{NA}$ \\
\hline \multirow{2}{*}{$\begin{array}{l}\text { Trauma vs. } \\
\text { Cardiac } \\
\text { Arrest }^{\mathrm{a}}\end{array}$} & $\begin{array}{l}\text { Adults/mixed- } \\
\text { age }\end{array}$ & $\begin{array}{l}\text { No conclusion } \\
\text { (Insufficient) }\end{array}$ & 2 OBS $^{61,104}$ & 15,924 & $0.97(0.71$ to 1.02$), 0 \%$ \\
\hline & Pediatrics & $\begin{array}{l}\text { No conclusion } \\
\text { (Insufficient) }\end{array}$ & $1 \mathrm{OBS}^{61}$ & 374 & $1.00(0.80$ to 1.26$), \mathrm{NA}$ \\
\hline $\begin{array}{l}\text { Nonarrest } \\
\text { vs. Cardiac } \\
\text { Arrest }^{\mathrm{a}}\end{array}$ & $\begin{array}{l}\text { Adults/mixed- } \\
\text { age }\end{array}$ & $\begin{array}{l}\text { No difference } \\
\text { (Low) }\end{array}$ & $5 \mathrm{OBS}^{80,84,88,100,103}$ & 14,148 & $\begin{array}{l}1.00(0.87 \text { to } 1.15) \text {, } \\
96.1 \%\end{array}$ \\
\hline \multirow{2}{*}{$\begin{array}{l}\text { Trauma vs. } \\
\text { Medical }^{\mathrm{b}}\end{array}$} & $\begin{array}{l}\text { Adults/mixed- } \\
\text { age }\end{array}$ & $\begin{array}{l}\text { Favors medical } \\
\text { (Low) }\end{array}$ & 4 OBS $^{61,74,101,104}$ & 9,527 & 0.96 (0.93 to 0.99$), 0 \%$ \\
\hline & Pediatrics & $\begin{array}{l}\text { No conclusion } \\
\text { (Insufficient) }\end{array}$ & $1 \mathrm{OBS}^{61}$ & 157 & $1.03(0.79$ to 1.35$), \mathrm{NA}$ \\
\hline
\end{tabular}

$\mathrm{CI}=$ confidence interval; $\mathrm{ETI}=$ endotracheal intubation; $\mathrm{I}^{2}=$ statistical test of heterogeneity; NA = not applicable; OBS = observational study; $\mathrm{SOE}=$ strength of evidence; $\mathrm{RR}=$ risk ratio

${ }^{\text {a }}$ Referent group $=$ cardiac arrest

${ }^{\mathrm{b}}$ Referent group $=$ medical

Table 39. Overall success for comparisons of emergency type as modifier of ETI

\begin{tabular}{|l|l|l|l|l|l|}
\hline Comparison & Subgroup & $\begin{array}{l}\text { Conclusion } \\
\text { (SOE) }\end{array}$ & $\begin{array}{l}\text { Study Number } \\
\text { and Design }\end{array}$ & $\begin{array}{l}\text { Number of } \\
\text { Patients }\end{array}$ & $\begin{array}{l}\text { Summary of Results } \\
\text { RR (95\% Cl), } \mathbf{I}^{\mathbf{2}}\end{array}$ \\
\hline $\begin{array}{l}\text { Medical vs. } \\
\text { Cardiac Arrest }\end{array}$ & $\begin{array}{l}\text { Adults/mixed- } \\
\text { age }\end{array}$ & $\begin{array}{l}\text { No difference } \\
\text { (Low) }\end{array}$ & 2 OBS 67,104 & 41,718 & $\begin{array}{l}0.88(0.66 \text { to 1.15), } \\
93.4 \%\end{array}$ \\
\hline $\begin{array}{l}\text { Trauma vs. } \\
\text { Cardiac Arrest }\end{array}$ & $\begin{array}{l}\text { Adults/mixed- } \\
\text { age }\end{array}$ & $\begin{array}{l}\text { No difference } \\
\text { (Low) }\end{array}$ & 3 OBS $^{67,102,104}$ & 31,586 & $0.97(0.89$ to 1.07$), 0 \%$ \\
\hline $\begin{array}{l}\text { Nonarrest vs. } \\
\text { Cardiac Arrest }\end{array}$ & $\begin{array}{l}\text { Adults/mixed- } \\
\text { age }\end{array}$ & $\begin{array}{l}\text { No difference } \\
\text { (Low) }\end{array}$ & 5 OBS $^{80,84,88,100,103}$ & 14,259 & $\begin{array}{l}0.98(0.90 \text { to } 1.07), \\
99.2 \%\end{array}$ \\
\hline $\begin{array}{l}\text { Trauma vs. } \\
\text { Medical }^{\mathbf{b}}\end{array}$ & $\begin{array}{l}\text { Adults/mixed- } \\
\text { age }\end{array}$ & $\begin{array}{l}\text { No difference } \\
\text { Low) }\end{array}$ & 3 OBS $^{67,101,104}$ & 17,969 & $1.00(0.99$ to 1.01), 0\% \\
\hline
\end{tabular}

$\mathrm{CI}=$ confidence interval; ETI = endotracheal intubation; $\mathrm{I}^{2}$ = statistical test of heterogeneity; OBS = observational study; RR = risk ratio; $\mathrm{SOE}=$ strength of evidence

${ }^{a}$ Referent group $=$ cardiac arrest

${ }^{b}$ Referent group $=$ medical 


\section{Patient Characteristics: Age, Sex, and Race}

Age

Results for comparisons of age as a modifier of ETI are summarized in Tables 40 and 41 . We included three categories for comparisons of age: age within pediatrics, pediatric versus adult, and elderly versus adult. We used the age categories as defined in each study; these thresholds varied across studies and are listed in the paragraphs below for the respective comparisons.

Three studies (in four publications; $\mathrm{N}=1,211$ ) provided data on comparative outcomes, including harms, for different ages within pediatric samples. ${ }^{35,36,105,106}$ Age cutoffs for the categories varied for the three studies. The thresholds in Eich, $2009^{105}$ were: infants less than 1 year old, toddlers age 1 to 5 years, and school-age children 6 to 14 years old. Prekker, $2016^{106}$ used these same cutoffs for infants and toddlers, with school age defined as 6 to 12 years old. Gausche, 2000 35,36 also had three age groups: age less than 3 years, 3 to 8 years old, and greater than 8 years old. Compared to toddlers or school-age children, infants had poorer outcomes for survival, neurological function and first-pass success. ${ }^{105,106}$ However, aspiration pneumonia occurred less frequently in infants than in children of other ages. ${ }^{106}$ For overall success, there were no differences across all pediatric age groups. ${ }^{35,36}$ There were no differences for toddlers versus school-age children for any outcome. ${ }^{105,106}$ Harms were experienced at similar rates for all age groups within pediatrics (Table 41). ${ }^{105,106}$

There were six studies $(\mathrm{N}=90,657)$ reporting on outcomes for pediatric versus adult patients. ${ }^{61,67,74,79,107,108}$ In Murray, 2000, ${ }^{108}$ adolescents were 11 to 20 years old and younger adults were age 21 to 40 years. Powell, $2019^{79}$ defined pediatrics as less than 18 years old; pediatric was defined as 14 years and younger in Jarvis, 2019, ${ }^{61}$ and Eberlein, $2019^{74}$ did not provide age cutoffs. Rates of survival to hospital discharge were similar for adolescents and young adults. ${ }^{108}$ In two studies of patients with mixed emergency types and prehospital providers of varying levels, first-pass success rates were higher for adults than children. ${ }^{61,74}$ However, one study of trauma patients treated by advanced prehospital providers in a helicopter EMS system found no difference in first-pass or overall success rates for children versus adults. ${ }^{79}$

One RCT $(n=156)$ compared elderly (age $>60$ years) versus adult patients, finding significantly lower rates of favorable neurological outcomes in the elderly. ${ }^{39}$

Table 40. Outcomes for comparisons of age as modifier of ETI

\begin{tabular}{|c|c|c|c|c|}
\hline Comparison & Outcome & $\begin{array}{l}\text { Study } \\
\text { Number and } \\
\text { Design }\end{array}$ & $\begin{array}{l}\text { Number of } \\
\text { Patients }\end{array}$ & Summary of Findings \\
\hline \multirow[t]{4}{*}{$\begin{array}{l}\text { Age Within } \\
\text { Pediatrics }\end{array}$} & Survival & $1 \mathrm{OBS}^{105}$ & 82 & $\begin{array}{l}\text { Prehospital and in-hospital survival: } \\
\text { - } \quad \text { Lower rates in infants vs. toddlers or school-age } \\
\text { children; prehospital: } 47.1 \% \text { vs. } 96.4 \% \text { vs. } 97.3 \% \\
\quad p<0.001 \text {; in-hospital: } 41.2 \% \text { vs. } 89.3 \% \text { vs. } 83.8 \% \text {, } \\
\quad \text { p }<0.001 \\
\text { No difference in toddlers vs. school-age children }\end{array}$ \\
\hline & $\begin{array}{l}\text { Neurologic } \\
\text { al function }\end{array}$ & $1 \mathrm{OBS}^{105}$ & 63 & $\begin{array}{l}\text { Pediatric CPC score } 1-3 \text { at hospital discharge: } \\
\text { - Smaller proportion of infants vs. toddlers or } \\
\text { school-age children; } 42.9 \% \text { vs. } 88 \% \text { vs } 93.5 \%, \\
\quad p<0.001 \\
\text { - No difference for toddlers vs. school-age children }\end{array}$ \\
\hline & $\begin{array}{l}\text { First-pass } \\
\text { success }\end{array}$ & $1 \mathrm{OBS}^{106}$ & 299 & $\begin{array}{l}\text { - Lower rates in infants than toddlers or school-age } \\
\text { children; } 52.6 \% \text { vs. } 74.1 \% \text { vs. } 73.9 \% \\
\text { - } \quad \text { Similar rates in toddlers and school-age children }\end{array}$ \\
\hline & $\begin{array}{l}\text { Overall } \\
\text { success }\end{array}$ & $1 \mathrm{CCT}^{35,36}$ & 310 & No difference among children of different ages \\
\hline
\end{tabular}




\begin{tabular}{|c|c|c|c|c|}
\hline Comparison & Outcome & $\begin{array}{l}\text { Study } \\
\text { Number and } \\
\text { Design }\end{array}$ & $\begin{array}{l}\text { Number of } \\
\text { Patients }\end{array}$ & Summary of Findings \\
\hline & Morbidity & $1 \mathrm{OBS}^{106}$ & 219 & $\begin{array}{l}\text { Aspiration pneumonia: } \\
\text { - Lower rates in infants than toddlers or school-age } \\
\text { children; } 5 \% \text { vs. } 24 \% \\
\text { - Similar rates in toddlers and school-age children }\end{array}$ \\
\hline \multirow[t]{4}{*}{$\begin{array}{l}\text { Pediatric } \\
\text { vs. Adult }\end{array}$} & Survival & $1 \mathrm{OBS}^{108}$ & 57 & $\begin{array}{l}\text { Survival to hospital discharge: } \\
\text { - No difference between adolescents vs. younger } \\
\text { adults }\end{array}$ \\
\hline & $\begin{array}{l}\text { First-pass } \\
\text { success }\end{array}$ & $3 \mathrm{OBS}^{61,74,79}$ & 23,078 & $\begin{array}{l}\text { Inconsistent } \\
\text { - Lower rates in pediatrics vs. adults; } 50.0 \% \text { vs. } \\
81.8 \%, p=0.026 ; 7^{74} 58.5 \% \text { vs. } 72.7 \%, \text { AOR } 0.56 \\
(95 \% \mathrm{Cl} 0.46 \text { to } 0.67), p<0.05^{61} \\
\quad \text { No difference }^{79}\end{array}$ \\
\hline & $\begin{array}{l}\text { Overall } \\
\text { success }\end{array}$ & $2 \mathrm{OBS}^{67,107}$ & 47,441 & $\begin{array}{l}\text { Lower rates in pediatrics vs. adults } \\
\text { - } \quad 55.8 \% \text { vs. } 84.2 \%, p<0.05^{107} \\
-\quad 71.8 \% \text { vs. } 79.0 \%, p<0.05^{67}\end{array}$ \\
\hline & DASH-1A & $1 \mathrm{OBS}^{79}$ & 257 & No difference between pediatric and adult patients \\
\hline $\begin{array}{l}\text { Elderly vs. } \\
\text { Adult }\end{array}$ & $\begin{array}{l}\text { Neurologic } \\
\text { al function }\end{array}$ & $1 \mathrm{RCT}^{39}$ & 156 & $\begin{array}{l}\text { GOSe } 5-8 \text { at } 6 \text { months: } \\
\text { - Smaller proportion of elderly vs. adult; } 14 \% \text { vs. } \\
\quad 62 \%, p<0.001\end{array}$ \\
\hline
\end{tabular}

$\mathrm{AOR}=$ adjusted odds ratio; $\mathrm{CCT}=$ controlled clinical trial; $\mathrm{CI}=$ confidence interval; $\mathrm{ETI}=$ endotracheal intubation; $\mathrm{CPC}=$ Cerebral Performance Score; DASH-1A = Definitive Airway Sans Hypoxia/Hypotension on First Attempt; GOSe = Glasgow Outcome Scale extended; OBS = observational study; RCT = randomized control trial

Table 41. Harms for comparisons of age within pediatrics as modifier of ETI

\begin{tabular}{|c|c|c|c|c|}
\hline Harm Description & $\begin{array}{l}\text { Study } \\
\text { Design }\end{array}$ & $\begin{array}{l}\text { Number of } \\
\text { Patients }\end{array}$ & $\begin{array}{l}\text { Age Range: Proportion } \\
\text { Experienced Harm }\end{array}$ & Summary of Findings \\
\hline $\begin{array}{l}\text { Peri-Intubation } \\
\text { Cardiac Arrest }\end{array}$ & $\mathrm{OBS}^{106}$ & 299 & $\begin{array}{l}<1 \text { year: } 2 \%(2 / 114) \\
1 \text { to } 5 \text { years: } 1 \%(1 / 116) \\
6 \text { to } 12 \text { years: } 4 \%(3 / 69)\end{array}$ & No difference across age groups \\
\hline \multirow{2}{*}{$\begin{array}{l}\text { Unrecognized } \\
\text { Bronchial Intubation }\end{array}$} & $\mathrm{OBS}^{106}$ & 241 & $\begin{array}{l}<1 \text { year: } 16 \%(12 / 75) \\
1 \text { to } 5 \text { years: } 24 \%(26 / 108) \\
6 \text { to } 12 \text { years: } 14 \%(9 / 64)\end{array}$ & No difference across age groups \\
\hline & $\mathrm{OBS}^{105}$ & 58 & $\begin{array}{l}<1 \text { year: } 0 \%(0 / 13) \\
1 \text { to } 5 \text { years: } 4.5 \%(1 / 22) \\
6 \text { to } 14 \text { years: } 4.3 \%(1 / 23)\end{array}$ & $\begin{array}{l}\text { No difference between any age } \\
\text { groups }\end{array}$ \\
\hline $\begin{array}{l}\text { Respiratory Tract } \\
\text { Injury }\end{array}$ & $\mathrm{OBS}^{106}$ & 295 & $\begin{array}{l}<1 \text { year: } 0 \%(0 / N R) \\
1 \text { to } 5 \text { years: } 1 \%(1 / N R) \\
6 \text { to } 12 \text { years: } 0 \%(0 / N R)\end{array}$ & Single case observed \\
\hline
\end{tabular}

ETI = endotracheal intubation; $\mathrm{NR}=$ not reported; $\mathrm{OBS}=$ observational study

\section{Sex}

Five observational studies $(\mathrm{N}=31,219)$ were included for comparative outcomes by patient sex (female vs. male) as a modifier of ETI (Table 42). ${ }^{61,74,79,109}$ Better outcomes were observed for females for survival and sustained ROSC. ${ }^{78,109}$ There were higher rates of first-pass success in females, ${ }^{61,74,79}$ however these differences were only statistically significant in one of three studies ${ }^{61}$ There was no difference in rates of overall success or DASH-1A for females and males. ${ }^{78,79}$ No eligible studies provided data on comparative harms for sex. 
Table 42. Outcomes for comparisons of sex as modifier of ETI

\begin{tabular}{|c|c|c|c|c|}
\hline Comparison & Outcome & $\begin{array}{l}\text { Study Number } \\
\text { and Design }\end{array}$ & $\begin{array}{l}\text { Number of } \\
\text { Patients }\end{array}$ & Summary of Findings \\
\hline \multirow{5}{*}{$\begin{array}{l}\text { Female vs. } \\
\text { Male }\end{array}$} & Survival & $1 \mathrm{OBS}^{109}$ & 299 & $\begin{array}{l}\text { Survival to hospital discharge: } \\
\text { - Higher rates in female vs. male } \\
\text { participants; AOR } 1.69(95 \% \mathrm{Cl} 1.10 \\
\text { to } 2.63), \mathrm{p}<0.05\end{array}$ \\
\hline & ROSC & $1 \mathrm{OBS}^{109}$ & 1,142 & $\begin{array}{l}\text { Sustained ROSC to hospital arrival: } \\
\text { - } \quad \text { Larger proportion of female vs. male } \\
\text { participants; AOR } 1.52(95 \% \mathrm{Cl} 1.12 \\
\text { to } 2.04), \mathrm{p}<0.05\end{array}$ \\
\hline & DASH-1A & $1 \mathrm{OBS}^{79}$ & 257 & No difference \\
\hline & $\begin{array}{l}\text { First-pass } \\
\text { success }\end{array}$ & $3 \mathrm{OBS}^{61,74,79}$ & 23,078 & $\begin{array}{l}\text { Inconsistent } \\
\text { - } \quad \text { No difference }{ }^{74,79} \\
\text { - } \quad \text { Higher rates in female vs. male } \\
\text { participants; } 74.7 \% \text { vs. } 70.8 \%, \\
\text { p<0.00161 }\end{array}$ \\
\hline & Overall success & $1 \mathrm{OBS}^{78}$ & 150 & No difference \\
\hline
\end{tabular}

AOR = adjusted odd ratio; $\mathrm{CI}=$ confidence interval; DASH-1A = Definitive Airway Sans Hypoxia/Hypotension on First Attempt; ETI = endotracheal intubation; OBS = observational study; ROSC $=$ return of spontaneous circulation

Race

Three studies $(\mathrm{N}=30,773)$ conducted in the United States provided data for outcomes by patient race as a modifier of ETI, but were limited to comparisons of white versus nonwhite race (Table 43) ${ }^{61,79,109}$ White participants had higher rates of sustained ROSC when compared to participants of nonwhite race. ${ }^{109}$ One large observational study found a statistically significant association between white race and first-pass success. ${ }^{61}$ A separate study found no difference in first-pass success rates, but reported higher rates of DASH-1 A in white participants; of note, more than 95 percent of the participants in this study identified as white. ${ }^{79}$ No eligible studies provided data on comparative harms by race.

Table 43. Outcomes for comparisons of race as modifier of ETI

\begin{tabular}{|c|c|c|c|c|}
\hline Comparison & Outcome & $\begin{array}{l}\text { Study Number } \\
\text { and Design }\end{array}$ & $\begin{array}{l}\text { Number of } \\
\text { Patients }\end{array}$ & Summary of Findings \\
\hline \multirow{3}{*}{$\begin{array}{l}\text { White vs. } \\
\text { Nonwhite }\end{array}$} & ROSC & $1 \mathrm{OBS}^{109}$ & 1,142 & $\begin{array}{l}\text { Sustained ROSC: } \\
\text { - Larger proportion of white participants than } \\
\text { nonwhite participants; AOR } 1.39(95 \% \mathrm{Cl} 1.04 \\
\text { to } 1.86), p<0.05\end{array}$ \\
\hline & DASH-1A & $1 \mathrm{OBS}^{79}$ & 257 & $\begin{array}{l}\text { Higher rates in white participants than nonwhite } \\
\text { participants; AOR } 4.62(95 \% \mathrm{Cl} 1.13 \text { to } 19.0) \text {, } \\
p=0.034\end{array}$ \\
\hline & $\begin{array}{l}\text { First-pass } \\
\text { success }\end{array}$ & $2 \mathrm{OBS}^{61,79}$ & 29,631 & $\begin{array}{l}\text { Inconsistent } \\
\text { - Similar rates for white and nonwhite } \\
\text { participants }{ }^{79} \\
\text { White race associated with higher rates of } \\
\text { first-pass success }{ }^{61}\end{array}$ \\
\hline
\end{tabular}

AOR = adjusted odds ratio; $\mathrm{CI}=$ confidence interval; ETI = endotracheal intubation; DASH-1A = Definitive Airway Sans

Hypoxia/Hypotension on First Attempt; OBS = observational study; ROSC = return of spontaneous circulation 


\section{Supraglottic Airway}

\section{Supraglottic Airway: Technique/Device}

\section{Technique/Device: Supraglottic Airway Devices}

We identified six studies that compared SGA devices. ${ }^{16,64,110-113}$ SGA devices were classified based on the anatomic position of their seal (e.g., pharyngeal or perilaryngeal) and results were grouped by comparisons within or between these classes.

\section{Perilaryngeal Seal Supraglottic Airways}

Two RCTs $(\mathrm{N}=454)$ were included for the comparison of perilaryngeal seal SGAs (Table 44). ${ }^{110,111}$ Both studies compared i-gel to LMA in adult cardiac arrest patients. There were higher rates of overall success with i-gel in the smaller RCT. ${ }^{111}$ No differences were found for survival to hospital discharge, ROSC on hospital arrival, or first-pass success.

Table 44. Outcomes for comparisons of perilaryngeal seal SGAs

\begin{tabular}{|c|c|c|c|c|}
\hline Comparison & Outcome & $\begin{array}{l}\text { Study Number } \\
\text { and Design }\end{array}$ & $\begin{array}{l}\text { Number of } \\
\text { Patients }\end{array}$ & Summary of Findings \\
\hline \multirow{4}{*}{ i-gel vs. LMA } & Survival & $1 \mathrm{RCT}^{110}$ & 406 & $\begin{array}{l}\text { Survival to hospital discharge: } \\
\text { - No difference }\end{array}$ \\
\hline & ROSC & $2 \mathrm{RCT}^{110,111}$ & 454 & $\begin{array}{l}\text { ROSC on ED or hospital arrival } \\
\text { - No difference }\end{array}$ \\
\hline & First-pass success & $1 \mathrm{RCT}^{110}$ & 406 & No difference \\
\hline & Overall success & $1 \mathrm{RCT}^{111}$ & 48 & $\begin{array}{l}\text { Higher rates with i-gel; } 90 \% \text { vs. } 57 \% \text {, RR } \\
1.58(95 \% \mathrm{Cl} 1.11 \text { to } 2.24), p=0.023\end{array}$ \\
\hline
\end{tabular}

$\mathrm{CI}=$ confidence interval; $\mathrm{ED}=$ emergency department; LMA = laryngeal mask airway; $\mathrm{RCT}=$ randomized control trial; $\mathrm{RR}=$ risk ratio; $\mathrm{ROSC}=$ return of spontaneous circulation; $\mathrm{SGA}=$ supraglottic airway

\section{Pharyngeal Versus Perilaryngeal Seal Supraglottic Airways}

Four studies (3 RCTs,,${ }^{16,12,113} 1$ observational; $\left.{ }^{64} \mathrm{~N}=123,692\right)$ provided data for comparisons of pharyngeal versus perilaryngeal seal SGAs (Tables 45 and 46). All studies were in adult patients. Pharyngeal seal SGAs included LT, esophageal obturators, Combitube, and PTLA. The perilaryngeal seal SGA in all studies was the LMA.

For ROSC, findings were inconsistent between studies. In one observational study, there was a statistically significant difference in rates of prehospital ROSC, favoring the pharyngeal seal SGA; however, the difference is not clinically meaningful (4.41\% using esophageal obturator airway vs. $4.90 \%$ using LMA) ${ }^{64}$ In RCTs, there was no difference between device types (LT vs. LMA) for either prehospital ROSC ${ }^{113}$ or overall ROSC. ${ }^{112}$ There were also inconsistent findings for successful airway insertion. First-pass and overall success rates were higher with perilaryngeal seal SGA in one RCT, ${ }^{113}$ while another $\mathrm{RCT}^{16}$ found higher rates of successful insertion and ventilation by EMS personnel assessment with pharyngeal seal SGAs. No differences were found for survival, ${ }^{64,112}$ neurological function, ${ }^{64,112}$ or oxygenation/ventilation ${ }^{16,112}$ outcomes.

Comparative harms for pharyngeal versus perilaryngeal seal SGAs were reported in two RCTs (Table 46). ${ }^{16,113}$ Where there were differences, higher rates of harms were experienced with use of pharyngeal seal SGAs (total complications, inadequate ventilation, and dislodgement). 
Table 45. Outcomes for comparisons of pharyngeal versus perilaryngeal seal SGAs

\begin{tabular}{|c|c|c|c|c|}
\hline Outcome & Comparison & $\begin{array}{l}\text { Study Number } \\
\text { and Design }\end{array}$ & $\begin{array}{l}\text { Number of } \\
\text { Patients }\end{array}$ & Summary of Findings \\
\hline \multirow[b]{2}{*}{ Survival } & LT vs. LMA & $1 \mathrm{RCT}^{112}$ & 313 & $\begin{array}{l}\text { Survival at } 1 \text { month } \\
\text { - No difference }\end{array}$ \\
\hline & $\begin{array}{l}\text { Esophageal } \\
\text { obturatora vs. LMA }^{\text {abtura }}\end{array}$ & $1 \mathrm{OBS}^{64}$ & 122,194 & $\begin{array}{l}\text { Survival at } 1 \text { month: } \\
\text { - } \quad \text { No difference }\end{array}$ \\
\hline \multirow{2}{*}{$\begin{array}{l}\text { Neurological } \\
\text { Function }\end{array}$} & LT vs. LMA & $1 \mathrm{RCT}^{112}$ & 313 & $\begin{array}{l}\text { CPC score } 1 \text { or } 2 \text { at } 1 \text { month: } \\
\text { - No difference }\end{array}$ \\
\hline & $\begin{array}{l}\text { Esophageal } \\
\text { obturatora vs. LMA }^{\text {of }}\end{array}$ & $1 \mathrm{OBS}^{64}$ & 122,194 & $\begin{array}{l}\text { CPC score } 1 \text { or } 2 \text { at } 1 \text { month: } \\
\text { - No difference }\end{array}$ \\
\hline \multirow[t]{2}{*}{ ROSC } & LT vs. LMA & $2 \mathrm{RCT}^{112,113}$ & 1,213 & $\begin{array}{l}\text { Prehospital ROSC: }{ }^{113} \\
\text { - No difference } \\
\text { Overall ROSC:112 } \\
\text { - No difference }\end{array}$ \\
\hline & $\begin{array}{l}\text { Esophageal } \\
\text { obturator }^{\mathrm{a}} \text { vs. LMA }\end{array}$ & $1 \mathrm{OBS}^{64}$ & 122,194 & $\begin{array}{l}\text { Prehospital ROSC } \\
\text { - } \quad \text { Favors esophageal obturator; } \\
4.41 \% \text { vs. } 4.90 \%, p=0.0002 \\
\end{array}$ \\
\hline \multirow[b]{2}{*}{ Success } & LT vs. LMA & $1 \mathrm{RCT}^{113}$ & 900 & $\begin{array}{l}\text { First-pass and overall success: } \\
\text { - } \quad \text { Higher rates with LMA, } p<0.02\end{array}$ \\
\hline & Combitube vs. LMA & $1 \mathrm{RCT}^{16}$ & 185 & $\begin{array}{l}\text { Successful insertion and ventilation: } \\
\text { - Higher rate with Combitube, } \\
p=0.048\end{array}$ \\
\hline \multirow{2}{*}{$\begin{array}{l}\text { Oxygenation/ } \\
\text { Ventilation }\end{array}$} & LT vs. LMA & $1 \mathrm{RCT}^{112}$ & 313 & $\begin{array}{l}\text { Successful ventilation at time of } \\
\text { hospital arrival: } \\
\text { - No difference }\end{array}$ \\
\hline & PTLA vs. LMA & $1 \mathrm{RCT}^{16}$ & 100 & $\begin{array}{l}\mathrm{ABG} \text { at hospital arrival, mean } \mathrm{PCO}_{2} \\
\text { and mean } \mathrm{PO}_{2} \text { : } \\
\text { - } \quad \text { No difference }\end{array}$ \\
\hline
\end{tabular}

$\mathrm{ABG}=$ arterial blood gas; $\mathrm{CPC}=$ Cerebral Performance Category; LMA = laryngeal mask airway; $\mathrm{LT}=$ laryngeal tube; $\mathrm{OBS}=$ observational study; PTLA = pharyngeotracheal lumen airway; RCT = randomized control trial; ROSC = return of spontaneous circulation; SGA = supraglottic airway

${ }^{a}$ Esophageal obturator included LT, Combitube, or esophageal gastric tracheal airway

Table 46. Harms for comparisons of pharyngeal versus perilaryngeal seal SGAs

\begin{tabular}{|c|c|c|c|c|c|c|}
\hline $\begin{array}{l}\text { Harm } \\
\text { Description }\end{array}$ & Comparison & $\begin{array}{l}\text { Study } \\
\text { Design }\end{array}$ & $\begin{array}{l}\text { Number } \\
\text { of } \\
\text { Patients }\end{array}$ & $\begin{array}{l}\text { Proportion } \\
\text { Experienced } \\
\text { Harm for: }\end{array}$ & $\begin{array}{l}\text { Versus } \\
\text { Proportion } \\
\text { Experienced } \\
\text { Harm for: } \\
\end{array}$ & Summary of Findings \\
\hline Complications & LT vs. LMA & $\mathrm{RCT}^{113}$ & 571 & $\begin{array}{l}\text { LT: } 19.0 \% \\
(33 / 174)\end{array}$ & $\begin{array}{l}\text { LMA: } 8.6 \% \\
(34 / 397)\end{array}$ & $\begin{array}{l}\text { Higher rate with LT; } \\
\text { AOR } 2.71(95 \% \mathrm{Cl} \\
1.69 \text { to } 4.35), p<0.001\end{array}$ \\
\hline $\begin{array}{l}\text { Tonguel } \\
\text { Pharyngeal } \\
\text { Swelling }\end{array}$ & LT vs. LMA & $\mathrm{RCT}^{113}$ & 571 & $\begin{array}{l}\text { LT: } 1.1 \% \\
(2 / 174)\end{array}$ & $\begin{array}{l}\text { LMA: } 1.0 \% \\
(4 / 397)\end{array}$ & No difference \\
\hline Bleeding & LT vs. LMA & $\mathrm{RCT}^{113}$ & 571 & $\begin{array}{l}\text { LT: } 2.3 \% \\
(4 / 174)\end{array}$ & $\begin{array}{l}\text { LMA: } 1.5 \% \\
(6 / 397)\end{array}$ & No difference \\
\hline Air Leak & LT vs. LMA & $\mathrm{RCT}^{113}$ & 571 & $\begin{array}{l}\text { LT: } 1.7 \% \\
(3 / 174)\end{array}$ & $\begin{array}{l}\text { LMA: } 0.5 \% \\
(2 / 397)\end{array}$ & No difference \\
\hline $\begin{array}{l}\text { Incorrect } \\
\text { Placement }\end{array}$ & LT vs. LMA & $\mathrm{RCT}^{113}$ & 571 & $\begin{array}{l}\text { LT: } 2.3 \% \\
(4 / 174)\end{array}$ & $\begin{array}{l}\text { LMA: } 1.3 \% \\
(5 / 397)\end{array}$ & No difference \\
\hline $\begin{array}{l}\text { Inadequate } \\
\text { Ventilation }\end{array}$ & $\begin{array}{l}\text { Combitube } \\
\text { vs. LMA }\end{array}$ & $\mathrm{RCT}^{16}$ & 185 & $\begin{array}{l}\text { Combitube: } \\
23 \%(18 / 77)\end{array}$ & $\begin{array}{l}\text { LMA: } 7 \% \\
(8 / 108)\end{array}$ & $\begin{array}{l}\text { Higher rate with } \\
\text { Combitube, } p=0.01\end{array}$ \\
\hline Dislodgement & LT vs. LMA & $\mathrm{RCT}^{113}$ & 571 & $\begin{array}{l}\text { LT: } 12.6 \% \\
(22 / 174)\end{array}$ & $\begin{array}{l}\text { LMA: } 4.5 \% \\
(18 / 397)\end{array}$ & $\begin{array}{l}\text { Higher rate with LT, } \\
p=0.001\end{array}$ \\
\hline
\end{tabular}

$\mathrm{AOR}=$ adjusted odds ratio; $\mathrm{CI}=$ confidence interval; LMA = laryngeal mask airway; $\mathrm{LT}=$ laryngeal tube; PTLA = pharyngeotracheal lumen airway; RCT = randomized control trial; SGA = supraglottic airway 


\section{Pharyngeal Seal Supraglottic Airways}

A single RCT ( $\mathrm{n}=194)$ compared pharyngeal seal SGAs (Combitube versus PTLA). ${ }^{16}$ There were no differences between devices for successful insertion and ventilation or arterial blood gases (Table 47). For harms, similar rates were observed for inadequate ventilation on hospital arrival (Table 48).

Table 47. Outcomes for comparisons of pharyngeal seal SGAs

\begin{tabular}{|c|c|c|c|c|}
\hline Comparison & Outcome & $\begin{array}{l}\text { Study Number } \\
\text { and Design }\end{array}$ & $\begin{array}{l}\text { Number of } \\
\text { Patients }\end{array}$ & Summary of Findings \\
\hline \multirow[b]{2}{*}{$\begin{array}{l}\text { Combitube vs. } \\
\text { PTLA }\end{array}$} & Success & $1 \mathrm{RCT}^{16}$ & 194 & $\begin{array}{l}\text { Successful insertion and ventilation: } \\
\text { - No difference }\end{array}$ \\
\hline & $\begin{array}{l}\text { Oxygenation/ } \\
\text { ventilation }\end{array}$ & $1 \mathrm{RCT}^{16}$ & 66 & $\begin{array}{l}\text { ABG at hospital arrival, mean } \mathrm{PCO}_{2} \text { and } \\
\text { mean } \mathrm{PO}_{2} \text { : } \\
\text { - } \quad \text { No difference }\end{array}$ \\
\hline
\end{tabular}

ABG = arterial blood gas; PTLA = pharyngeotracheal lumen airway; RCT = randomized control trial; SGA = supraglottic airway

Table 48. Harms for Combitube versus PTLA as modifier of SGA

\begin{tabular}{|l|l|l|l|l|l|}
\hline Harm Description & Study Design & $\begin{array}{l}\text { Number of } \\
\text { Patients }\end{array}$ & $\begin{array}{l}\text { Proportion } \\
\text { Experienced } \\
\text { Harm: Combitube }\end{array}$ & $\begin{array}{l}\text { Proportion } \\
\text { Experienced } \\
\text { Harm: PTLA }\end{array}$ & $\begin{array}{l}\text { Summary of } \\
\text { Findings }\end{array}$ \\
\hline $\begin{array}{l}\text { Inadequate } \\
\text { Ventilation }\end{array}$ & 194 & $23 \%(18 / 77)$ & $20 \%(23 / 117)$ & No difference \\
\hline
\end{tabular}

PTLA = pharyngeotracheal lumen airway; RCT = randomized control trial; SGA = supraglottic airway

\section{Supraglottic Airway: Patient Characteristics}

\section{Patient Characteristics: Age}

Two large observational studies were included for age (pediatric versus adult) as a modifier of SGA outcomes (Table 49). ${ }^{61,67}$ Studies utilized multiple devices including both pharyngeal and perilaryngeal seal SGAs. The rates of first-pass success and overall success were not different for children compared to adults. No eligible studies provided data on comparative harms for age as a modifier of SGA.

Table 49. Outcomes for comparisons of age as modifier of SGA: pediatric versus adult

\begin{tabular}{|l|l|r|l|}
\hline Outcome & Study Number and Design & Number of Patients & Summary of Findings \\
\hline First-Pass Success & 1 OBS $^{61}$ & 6,849 & No difference \\
\hline Overall Success & O OBS $^{67}$ & 9,461 & No difference \\
\hline
\end{tabular}

OBS = observational study; SGA = supraglottic airway 


\section{Discussion}

\section{Findings in Relation to the Decisional Dilemmas}

\section{Introduction}

An essential part of prehospital care is airway management, which enables patients to receive adequate oxygenation and ventilation. There are currently three main approaches to airway management: bag valve mask (BVM) (usually with airway adjuncts such as oropharyngeal airway and nasopharyngeal airway), supraglottic airway (SGA), and endotracheal intubation (ETI). While guidelines and best practices exist, individual experiences, policies, and research do not definitively support one airway approach over another. Furthermore, these approaches are often used in a complementary fashion so that one serves as a backup when the other is deemed ineffective.

Determining individual patient needs in the prehospital environment is challenging, and the actions first responders take are influenced by myriad factors that can vary significantly across patient and clinical scenarios. An essential factor is the variation in resources available for prehospital care, including modes of transport (e.g., ground vs. air), level of training and expertise of the prehospital clinician, and available equipment on scene. Additional factors influencing emergency practitioner actions include the specific clinical patient scenario, and estimated transport time to an emergency department and hospital. These can also change dynamically throughout emergency medical services (EMS) calls. In this review, our objective was to aggregate and summarize findings regarding these factors to facilitate application in local environments.

Our quantitative and qualitative syntheses were based on 99 studies from 101 publications. Studies compared BVM to SGA (Key Question 1), BVM to ETI (Key Question 2), SGA to ETI (Key Question 3), and selected modifiers within BVM, SGA, or ETI (Key Question 4). The aim of the quantitative syntheses was to identify any differences in survival in-hospital or at 1-month post incident, neurological function at discharge or 1-month post incident, return of spontaneous circulation (ROSC), or successful advanced airway insertion. Results were stratified by emergency type and age, since patient needs and clinical presentation across emergency types and age differ to the degree that it was not clinically reasonable to combine them. Key results are reported in Tables 3, 4, and 5 .

The included studies were primarily observational and limited by indication and survival bias; very few randomized controlled trials (RCTs) were available. Most strength of evidence (SOE) assessments were "low," mainly due to the limited number of studies and inconsistencies in outcomes. Those outcomes rated "moderate" included more studies, more rigorous study designs, consistent findings, or more precise estimates. There were no "high" SOE ratings. Therefore, additional well-designed future studies could change our conclusions.

\section{Key Results}

\section{Key Questions 1, 2, and 3}

Results for Key Questions 1, 2, and 3 were quantitatively analyzed for survival, neurological function, and ROSC (Table 3); and for successful advanced airway insertion for Key Question 3 (Table 4). Overall, evidence indicated few differences between airway approaches; when 
statistically significant differences occurred, they were for specific outcomes/comparisons and didn't indicate a pattern clearly favoring one airway over another across multiple outcomes.

Our pooled estimates found few statistically significant differences in outcomes from headto-head comparisons of airway management methods across most subgroups of emergency types and ages.

For Key Question 1 (BVM vs. SGA), there was no difference in survival for adult/mixed-age patients with cardiac arrest, and pediatric patients with cardiac arrest; and no difference in ROSC for adult patients with cardiac arrest. For neurological function measured by the Cerebral Performance Category (CPC), there were better outcomes with BVM for adult/mixed-age patients with cardiac arrest. No differences in harms were noted. ${ }^{13,15,18,19}$

There was limited confidence in these findings, as it often was not clear whether the comparison was BVM versus SGA directly, or BVM versus BVM initially, followed by SGA insertion. Studies did not always clearly identify whether other devices (e.g., oropharyngeal and nasopharyngeal airway) were used in conjunction with BVM, or describe how BVM was actually performed (e.g., by one- vs. two-person technique). Finally, some studies assessed efficacy of BVM using chest rise and fall, which is not always measured reliably or consistently across providers. More objective measures of ventilation effectiveness, such as waveform capnography or tidal volume measurements, would be useful, as blood gas analysis is not practical in the field setting.

There was a strong possibility that resuscitation time bias influenced results favoring BVM. ${ }^{114}$ Resuscitation time bias refers to interventions that are applied at varying times; those applied later are less effective in part due to their delayed application. As BVM typically is the first airway management technique used in the field, effects of successful BVM would be favorably confounded by the shorter time between EMS arrival and airway intervention. This is particularly true for patients presenting with cardiac arrest with favorable features such as being witnessed, receiving bystander CPR, and a shockable initial rhythm. Another contributing factor is hyperventilation, which may occur more frequently with advanced airways (SGA or ETI) than with BVM. Hyperventilation has been shown to adversely impact patient outcomes in part by increasing intrathoracic pressure and decreasing venous return, ultimately leading to decreased cerebral and coronary perfusion pressures. ${ }^{115-119}$

For Key Question 2 (BVM vs. ETI), results indicated no difference in survival, neurological function measured by the CPC, or ROSC across the subgroups identified in Table 3. No differences in harms were noted. ${ }^{18,35-40}$ The same caveats apply as identified for Key Question 1 with respect to study limitations. For example, whether ETI was preceded by BVM or not, lack of precise details on BVM use and subjective measurement of its effectiveness, resuscitation time bias, and potential hyperventilation all limit firm conclusions for this question.

For Key Question 3 (SGA vs. ETI), outcomes favored SGA for ROSC in adults with cardiac arrest, ${ }^{17,21-23,28,29,33,44,46,51,52,55,62,64,65,68}$ and for first-pass success in adults with cardiac arrest, ${ }^{17,49,51,61,69}$ pediatric patients with cardiac arrest, ${ }^{18,61}$ and adults with mixed emergency types. ${ }^{53,63}$ Outcomes favored ETI for neurological function in adults with cardiac arrest (measured by the CPC). ${ }^{21-23,28,31,33,52,59,62,64,65}$ There was no difference between approaches for survival in adult/mixed-age patients with cardiac arrest ${ }^{14,17,21-25,28,30,32,44,46,52,62,64,65}$ and pediatric patients with cardiac arrest; ${ }^{18,26,66}$ for neurological function in adults with cardiac arrest (measured by the Modified Rankin Sore $[\mathrm{mRS}])^{17,44}$ and pediatric patients with cardiac arrest (measured by the Pediatric Cerebral Performance Category [PCPC] $) ;{ }^{26,66}$ for ROSC in pediatric patients with cardiac arrest; ${ }^{26,66}$ for first-pass success in adults with medical emergencies, ${ }^{45,61}$ and 
for overall successful advanced airway insertion in adults with cardiac arrest, ${ }^{17,44,46,49-51,58,67,69}$ adults with medical emergencies, ${ }^{45,48,67}$ and adults with mixed emergency types. ${ }^{53,54,56}$

For harms, better outcomes were observed with SGA for multiple insertion attempts, ${ }^{17}$ and with ETI for inadequate ventilation. ${ }^{17}$ No differences were noted for aspiration, ${ }^{44,63}$ oral/airway trauma, ${ }^{17,18}$ and regurgitation. ${ }^{44}$

Compared with ETI, SGAs were faster to insert, and had higher first-pass success in specific subgroups. However, no difference was noted in rates of overall insertion success. It is thought that SGAs may not protect against aspiration and thus may not work well for patients with vomiting, or fluid or blood in the airway. While overall rates of aspiration were similar between groups, aspiration may be more common during or after an advanced airway attempt with SGA as compared to ETI. Since the SGA is placed above the glottis, it may also be more difficult for EMS clinicians to hyperventilate with the SGA than with ETI. This is a topic for future research. From an EMS perspective, ROSC is the primary field resuscitation endpoint in cardiac arrest and therefore a meaningful outcome for first responders. Most studies report ROSC outcomes were improved with SGA versus ETI (Table 18). Survival and neurological function are influenced by postresuscitation care, including hospital procedures (e.g., targeted temperature management, cardiac catheterization, and critical care expertise) and shared decision making with family regarding prognosis and withdrawal of life sustaining treatments. Best practices regarding neuroprognostication are evolving, and unfortunately, at present patients may be moved too quickly to comfort care, especially following cardiac arrest. ${ }^{120}$

\section{Key Question 4}

No studies met inclusion criteria about modifiers for BVM. Studies addressing modifiers for SGA were analyzed qualitatively. For ETI, results were both pooled in meta-analyses and analyzed qualitatively (Table 5). For both SGA and ETI, modifiers included technique/device and patient characteristics.

\section{Endotracheal Intubation}

\section{Technique/Device}

There were four sets of comparisons for technique/device modifiers of ETI: ETI with drugfacilitation versus without; video versus direct laryngoscopy; laryngoscope blade material; and use of the gum elastic bougie as an adjunct.

For the drug-facilitation category, we compared rapid sequence intubation (RSI) versus no medication, RSI versus sedation-facilitated, and sedation-facilitated versus no medication. For RSI versus no medication, there was no difference for survival in adult/mixed-age patients with trauma $;^{70,72,73,83}$ for first-pass success in adult/mixed-age patients trauma; ${ }^{79,83}$ for overall success in adults with cardiac arrest; ${ }^{67,77}$ and overall success in adult/mixed-age patients with mixed emergency types. ${ }^{78,80,84}$ RSI was favored for first-pass success in adult/mixed-age patients with mixed emergency types, ${ }^{61,74,80,82,84}$ and for overall success in adults with trauma ${ }^{67,70}$ For RSI versus sedation-facilitated ETI, RSI was favored for first-pass success in adult/mixed-age patients with mixed emergency types. ${ }^{61,74,84}$ For sedation-facilitated ETI versus no medication, there was no difference for first-pass success in adult/mixed age patients with mixed emergency types. $^{61,74,84}$

For video versus direct laryngoscopy, there was no difference for first-pass success for adults with cardiac arrest ${ }^{51,85,87,88,93}$ and adult/mixed age patients with mixed emergency types, ${ }^{86,88-92}$ 
and for overall success in adults with cardiac arrest ${ }^{51,85,87,88,93}$ and adult/mixed age patients with mixed emergency types. ${ }^{86,88-90,94-96}$

For laryngoscope blade materials, there was no difference between reusable versus single-use blades for first-pass success. ${ }^{97}$ Metal blades were favored over plastic blades for first-pass successful advanced airway insertion. ${ }^{98,99}$

For gum elastic bougie, there was no difference for overall success. ${ }^{78}$ Use of bougie was favored for first-pass success ${ }^{79}$ and Definitive Airway Sans Hypoxia/Hypotension on First Attempt (DASH-1A). ${ }^{79}$

\section{Patient Characteristics}

We analyzed how outcomes of ETI varied by emergency type, age, sex, and race. For emergency type, in adults/mixed age patients there was better first-pass success for medical emergencies when compared to trauma ${ }^{61,74,101,104}$ and there was no significant difference for nonarrest versus cardiac arrest. ${ }^{80,84,88,100,103}$ Overall success was not significantly different between any emergency types in adults/mixed age patients. ${ }^{64,67,80,84,88,100-104}$

In many of these studies, it was not always clear how success was measured. If intubation success in a cardiac arrest patient is based on paramedic documentation without waveform capnography/video/independent confirmation, and the patient does not survive to hospital arrival, the intubation success cannot be confirmed. On the other hand, if the patient survives to hospital arrival, then it is more likely that the airway success will be confirmed.

For age, within pediatrics there were poorer outcomes for infants versus toddlers or schoolage children for survival, ${ }^{105}$ neurological function, ${ }^{105}$ and first-pass success, ${ }^{106}$ whereas there were better outcomes for infants versus toddlers or school-age children for aspiration pneumonia. ${ }^{106}$ There was no difference for overall success across pediatric groups, ${ }^{106}$ and no difference between toddlers versus school-age children for any outcome. ${ }^{105,106}$ When pediatric patients were compared with adults, there was no difference for survival ${ }^{108}$ or DASH-1A. ${ }^{79}$ When adults were compared with elderly patients, no difference was found for neurological function. ${ }^{39}$

For sex, outcomes favored females for survival, ${ }^{109}$ ROSC,,${ }^{109}$ and overall success. ${ }^{78}$ There was no difference for DASH-1A. ${ }^{79}$

For race, outcomes were poorer for nonwhite compared to white patients for $\operatorname{ROSC}^{109}$ and DASH-1A. ${ }^{109}$

\section{Supraglottic Airway}

\section{Technique/Device}

Within the category of technique/device, we compared within and between categories of perilaryngeal seal SGAs and pharyngeal seal SGAs. In the perilaryngeal seal SGA comparisons (i-gel versus laryngeal mask airway [LMA]), results favored i-gel over LMA for overall successful advanced airway insertion, ${ }^{111}$ but there was no difference for survival, ROSC, or firstpass success. ${ }^{110,111}$ In the pharyngeal versus perilaryngeal seal SGA comparisons (laryngeal tube [LT], esophageal obturator, Combitube, or pharyngeotracheal lumen airway [PTLA] vs. LMA), no difference was found for survival, ${ }^{64,112}$ neurological function, ${ }^{64,112}$ and ventilation and arterial blood gases. ${ }^{16,112}$ In the pharyngeal seal SGA comparisons (Combitube versus PTLA), no difference was found for successful advanced airway insertion and arterial blood gases. ${ }^{16}$

Overall, these findings suggested that LMA may not be an ideal SGA device for EMS. The igel (with a similar shape as LMA) is technically less challenging to deploy given its cuffless 
design and is more effective in the prehospital environment. It appears to have higher leak pressures, provides a better seal, and has an intrinsic bite block, all of which may facilitate better ventilation.

\section{Patient Characteristics}

Within the category of patient characteristics, there was no difference between adults and pediatric patients for successful SGA insertion (first-pass or overall). ${ }^{61,67}$

\section{Summary of the Evidence}

The overall findings suggested that there are limited differences in patient-oriented outcomes between the three methods of airway management studied (BVM, SGA, and ETI), in particular survival to hospital discharge and survival with good neurological function. This is important because the level and extent of training required for the acquisition and retention of ETI skills is different than for the other techniques. The comparable performance of BVM and SGA, which have less training requirements, allows for effective airway management with oxygenation and ventilation to be provided for the majority of patients who need it.

The evidence did not suggest, in general, that outcomes improve using any one particular airway approach in any specific patient scenario. It is likely that having different methods available is also important since sometimes the circumstance calls for a particular strategy, even when all options are available to the provider. For example, ETI may be most appropriate in a patient with active vomiting or airway secretions in whom BVM (increases aspiration) or SGA (less protection against aspiration) are less than ideal.

Experience with airway management in the pediatric population is very limited across most EMS systems and skill maintenance is a constant challenge. This is also now increasingly true for adults since opportunities for training have become more limited over the past 20 years. In the past, prehospital providers could acquire initial and refresher training in the operating room, but this is no longer allowed in many hospitals. Given these challenges and similar patient orientated outcomes across airway management methods, EMS agencies need to reassess the importance and role of ETI in their approach to advanced airway management.

\section{Strengths and Limitations}

The results and conclusions detailed in this report have been shaped by the strengths and limitations of both the evidence available and our approach to the review. What questions researchers asked, how studies were designed, and what data were collected and reported establish the boundaries of what this systematic review can and cannot answer and our confidence in our conclusions. We made methodological choices and decisions about how to search for, analyze, and present this body of evidence that also impacted the report.

\section{Strengths and Limitations of the Evidence}

The primary strengths of the evidence base included the availability of prehospital studies that assessed important outcomes and the variety of interventions and indications. Additionally some, though not all, studies employed more rigorous designs.

We were able to identify 99 studies of prehospital airway management that compared the three types of airway approaches currently available (i.e., BVM, SGA, and ETI) or evaluated variations of a single approach. Responding to questions about prehospital care is often hindered 
by the fact that conducting research in the prehospital environment is challenging. When studying other elements of prehospital care, extrapolations have been made with evidence from emergency departments or simulations. A challenge was that the prehospital period is short and as a result the opportunities for data collection can be limited, so only short-term or intermediate outcomes, such as survival to hospital admission, were reported in studies. For this review, we were fortunate to have direct evidence consisting of prehospital studies that reported the key patient-centered outcomes of survival, neurological function and ROSC.

It was an advantage that the included studies were conducted in several different countries and that the research had wide variation in prehospital care situations, such as different types of emergencies, modes of transport available, and EMS system structure and personnel training. This review seeks to inform broad policies and guidelines for emergency prehospital care. If the body of evidence was limited to only a subset of the options, such as only air transport, only cardiac arrest patients, or only care in urban areas, applicability would be more limited.

This review included the results of 19 RCTs, 6 controlled clinical trials (CCTs), and 17 prospective cohort studies. The remaining studies were retrospective cohort studies and before/after comparisons. While there is no guarantee that trials and prospective studies provide better evidence, their ability to control or at least influence data collection and the delivery of care to some degree may reduce bias and confounding and increase the likelihood of including variables and outcomes needed to address the proposed research questions.

There were several important limitations to the available evidence assessing the impact of different airway devices in prehospital care. The most serious limitations resulted from the weaknesses of study designs and the risk of biases that are common challenges in prehospital and emergency care research. While the body of evidence did include trials, the majority are retrospective observational studies based on analyses of data from national or regional registries or administrative data from a single health system or EMS agency. This is not surprising as prospective studies and trials are more difficult, more costly, and subject to strict regulation, particularly as prehospital patients may be unable to consent to participate in the research. Bias may be more likely in observational studies, and this may explain why the results from trials and observational studies occasionally differ in this review. Indication bias, classifying patients by the treatment received, and survival bias, including only patients who survive a treatment are variants of selection bias that are likely to occur in observational studies of prehospital care. Furthermore, confounding variables can influence the observed outcomes. Measurement of confounders is often limited in large administrative databases, and analyses may not account for all relevant potential confounders. Other challenges may also introduce bias in both observational studies and trials in prehospital research. Specifically, prehospital care is provided in different patterns over the prehospital care time period with patients rarely receiving the exact treatment even within trial arms or treatment groups. Additionally, the impact of specific prehospital interventions may vary at different care time points, particularly when a patient's status is changing rapidly. ${ }^{121}$

Importantly, EMS clinicians acquire skill in all airway procedures over time and with practice. The skillset of the provider with each technique was rarely controlled for in the included studies for this review. It is likely that providers have greater skill with one technique more than others, which introduces another potential source of bias into the body of evidence.

Other limitations are specific to advanced airway management in the prehospital setting. In the field, use of more than one airway is typical with a progression through different approaches as the patient is assessed. The use of multiple airways, the order, and the duration of each may 
affect outcomes, but this information is rarely documented precisely and included in analyses. While some studies clearly define which airways were used first and when an airway was used as rescue when another airway failed, this is not explicit in all studies. Another concern is resuscitation time bias (i.e., the intervention is influenced by duration of resuscitation), and the patient's status and course of treatment preceding airway placement may influence both the intervention received and outcomes. ${ }^{114}$ The preparation time needed for different airway management techniques and the differences in skill and experience may be confounders, and the impact is often difficult to separate from the airway itself. An additional consideration is that there is variation in device designs within each class of airway. For example, SGA includes devices that seal in different locations and may or may not incorporate balloons in their design (e.g., LMA, King LT, and i-gel). The particular device used is specified in some studies, but even so, the variation in techniques and skill needed could contribute to variation in outcomes, and all possible comparisons within and across types of devices have not been studied.

Finally, there is a paucity of data regarding prehospital ventilation because we do not presently have a way to accurately measure it. When available we use waveform capnography, but this does not provide a complete picture and can be affected by other factors such as medications, tissue metabolism and blood flow to the lungs. The challenge with most airway trials to date is that they have not addressed what happens after the airway is secured. Ventilation has not been assessed consistently, so the differences noted in outcomes may be related to the ventilation provided and not the airway method. Better tools are needed to measure ventilation parameters in particular rate, tidal volume, and airway pressures.

\section{Strengths and Limitations of the Review}

The methods for this review were based on the Agency for Healthcare Research and Quality (AHRQ) methods guidance ${ }^{122}$ and the Institute of Medicine standards for systematic reviews. ${ }^{123}$ We searched multiple databases, requested Technical Expert Panel members, clinical experts, and reviewers to suggest known studies. We reviewed reference lists, and we solicited unpublished data and additional studies through the AHRQ public call for information. We utilized broad search strategies to increase our yield.

We limited our inclusion to studies in English, which may introduce bias, though we did not locate any English language abstracts of studies published in other languages that met our inclusion criteria. As we included observational studies, we were not able to assess some types of reporting bias, since most studies were not registered prior to their conduct in ClinicalTrials.gov or a similar registry. Additionally, some of the retrospective cohort studies were analyses of large trauma or emergency care registries. These registries contain data from multiple trauma centers or health systems. While we looked for potential overlap in the populations used in these studies and also for overlap between registry or multi-site studies and single site studies, we cannot be sure the populations are all mutually exclusive and it is possible that some patients were included more than once.

Using meta-analysis to pool the results requires judgements about what populations, interventions and outcomes are similar enough to combine and what subgroups are important. We established criteria a priori and have described this in our methods detail (see Appendix A). These decisions have an important impact on the results, and the results could differ if other criteria were used. A key decision we made was that all our results would be stratified by age group and emergency type. We did this based on our belief that combining these would be 
clinically inappropriate, although this limits the ability to make global statements about effectiveness.

\section{Applicability}

The applicability of the evidence we synthesized was operationalized in terms of how similar or different key aspects of the included studies correspond to the current practice and policy decisional dilemmas that inspired this review. Using the PICOS framework (Population, Intervention, Comparison, Outcome, Setting), we can identify what elements affect applicability and the extent to which the evidence available and the ideal research match.

Our assessment of the applicability of the synthesized evidence varies across PICOS elements. Some elements, such as the Population, Comparators, and Setting, mirror current practice and policy questions. Other elements of the research evidence, including details of interventions and the reported outcomes, are not sufficient to directly respond to the decisional dilemmas surrounding prehospital airway management, underscoring the needs for additional research as outlined later in this discussion.

The population of interest was all patients treated by emergency medical services for trauma, cardiac arrest, or other urgent causes of respiratory failure. All these types of patients as well as broad age categories of adults and pediatrics are represented in the included studies. This is important as prior evidence indicates that airway management is significantly different across these age groups. Other subgroup variation is not explicitly documented nor represented. For example, the race of patients is rarely reported in studies, making it difficult to determine if there are differences in needs, treatment, or outcomes and whether the results apply across racial groups. Unfortunately, the studies included for this review only provided two categories - white and nonwhite. The findings were consistent with what is seen in other cardiac arrest studies related to race and ethnicity and are likely not related to airway interventions. Nonwhite patients have poorer survival, possibly due to not having bystander actions, delays in calling 911, or other factors not yet addressed in the literature. ${ }^{124}$ Another influencing factor we do not have much information about is the race of the EMS clinician. Implicit bias may also affect how care is rendered in EMS but has not been studied in airway management.

While age was always reported, some studies did not report results by age groups. Other results suggested that patients may need to be further divided by age into finer groups as results differ for infants versus older children or for middle-aged adults versus older adults. Another characteristic of these patient populations that might be important in some cases, but is rarely reported, is pre-existing conditions. While many conditions may not be germane to airway decisions or the information may not be available, some such as obesity, can be observed, or others such as anticoagulant use could be communicated by medic alert bracelets or tags.

The comparators and setting of the studies also closely correspond to the Key Questions. The studies included direct comparisons of different types of airways or variations on a type of airway, corresponding to the decisional dilemma of what to recommend in practice. The studies were also all conducted in the field with actual patients, providing direct evidence. This eliminated the need to include studies conducted in the emergency department (ED) or simulations.

Most problematic for applicability are the known and unknown variations in key aspects of the airway interventions. As documented in Table 1, the included studies were conducted in several countries with different EMS systems. Key differences included the provision of prehospital care by physicians and the levels of training, scope of practice and supervision of 
nonphysician providers. For example, one of the more rigorous studies in this field is an RCT conducted in France and Belgium in which physicians were responsible for field ETI. ${ }^{37}$

Similarly, in Asian countries such as Japan, South Korea and Taiwan, prehospital providers are not permitted to place advanced airways without extra training and even then still are required to seek online medical control approval to place advanced airways. These differences reduced the applicability of results across systems. While we created functional categories for the level of emergency provider in each study, included this in our data abstraction (Appendix E), and listed the category on the meta-analysis forest plots next to each study (Appendix H and I), we did pool studies despite these variations and chose not to limit inclusion to studies conducted in the United States.

Applicability was also potentially impacted by differences in the outcomes measured and compared in the included studies. What is important continually changes as our understanding of physiology, physiologic reserve, and how the body responses evolve. We were encouraged to find studies that included outcomes that are patient-centered and not simply focused only on prehospital care processes. We believe survival, neurological function and ROSC are important as reflected in our focus on these outcomes for quantitative synthesis. However, there is an increasing emphasis on appropriate ventilation as a necessary precursor to better outcomes, and most studies did not provide data on ventilation. Specifically, leaders in emergency care are suggesting that the focus should be more on breathing than airway. This is in part due to the detrimental impact of hyperventilation for trauma and cardiac arrest patients and the higher probability that hyperventilation will occur when more attention is given to securing an airway then to ventilation and oxygenation. ${ }^{115-119}$ While a few studies did include blood gases on ED arrival and found no significant differences, these outcomes were not common. This sparsity of data means the current body of evidence cannot be easily applied to decisions about how to maximize these outcomes.

COVID-19: It is important to recognize that the systematic review and meta-analyses presented above were initiated prior to the COVID-19 pandemic, which has impacted airway management. As prehospital airway management is considered an aerosol-generating procedure, EMS providers must ensure they have appropriate respiratory, skin, and eye protection equipment to decrease potential exposures. Interim American Heart Association guidelines recommend that prehospital providers prioritize oxygenation and ventilation strategies with lower aerosolization risk, such as an endotracheal tube connected to a ventilator with a highefficiency particle air (HEPA) filter in the path of the exhaled gas. If available, video laryngoscopy may also help reduce intubation exposure to aerosolized particles. If endotracheal intubation is not feasible or delayed, manual ventilation with a BVM or SGA device should be performed with a HEPA filter in place.

\section{Implications for Clinical Practice, Education, Research, or Health Policy}

Based on the findings from this systematic review and meta-analysis, all three methods for airway management appeared to be effective options for patients in the prehospital environment. The preferred airway depends on setting, patient age and emergency type, and available provider expertise and equipment. As no method is universally successful, having all three available to support initial airway management attempts may optimize patient outcomes.

Effective airway management allows a means for oxygenation and ventilation. BVM and SGA, which are quicker to deploy, should be the mainstay management, as they facilitate prompt 
oxygenation and ventilation of the patient compared with ETI. Any device or technique that is used needs to be closely monitored (e.g., pulse oximetry, waveform capnography, future ventilation measuring devices) to ensure appropriate overall resuscitation.

\section{Provider Training, Expertise, and Skills Maintenance}

The quality of EMS performance in securing an airway will also influence patient outcomes. Paramedic training programs vary considerably in the United States in terms of number of hours, patient contact time, and live procedures. The opportunity for live training has diminished, as has the need for use of advanced airways since non-invasive ventilation is now an option for patients with hypoxemic and hypercarbic respiratory failure. This is an unfortunate reality faced by many training programs who have turned to other options such as cadaver and simulation settings to teach and verify skills prior to graduation. Our findings highlight the fact that good training programs need to teach a variety of skills related to airway management with the ultimate goal being oxygenation and ventilation. Skill maintenance, especially with advanced airway techniques, has also been a challenge, and some systems are restricting advanced skills like ETI to a smaller number of providers. ETI should be available, but requirements for continued skills maintenance and cost of equipment may necessitate limiting ETI to higher-level providers, especially in tiered systems where a more complex set of resources are sent to a smaller number of calls.

\section{Future Research}

Although airway management remains a key intervention in the prehospital setting, this systematic review and meta-analysis highlights several gaps in the literature, first being the need for more high-quality research from RCTs to minimize indication bias. While observational studies tend to be easier to conduct, they are often primarily hypothesis generating and provide a foundational basis for RCTs. Future airway studies need to clearly identify devices utilized and airway management methods (e.g., whether adjuncts were used with BVM [oropharyngeal and nasopharyngeal airway]) to allow for more accurate comparisons of the different airway methods). Future research needs to incorporate objective measures of success in oxygenation and ventilation (e.g., waveform capnography, video monitoring, in-line ventilation rate, flow, tidal volume, and pressure, etc.); newer monitors and developing technology will hopefully assist in more precise measurement of these outcomes. The ability to record procedures in real time is one of the key challenges faced by providers in the ED setting and data collection techniques and technologies have been developed. Consideration should be given to extending this into the prehospital setting. Resuscitation time bias remains an important issue in cardiac arrest studies, and efforts should be made to accurately capture airway intervention timing to mitigate this concern. Research is also needed to identify optimal methods to acquire and maintain airway management skills in the prehospital setting.

Specific recommendations include:

- Conduct RCTs that compare all three airway approaches in the same trial.

- Include data on oxygenation and ventilation, and assess effectiveness of the method of airway management to ventilate.

- Identify the impact of ventilation volume, ventilation rate, and airway pressure on outcomes across different airway methods to better understand the importance of airway versus breathing during resuscitation. 
- Clarify technique used in BVM studies (one person or two person, proper mask seal, airway adjuncts).

- Assess effects of experience as well as frequency of skill utilization with regards to ETI.

- Incorporate more objective outcome reporting methods, as observational studies often rely on self-reported success and failure.

- Conduct more research dedicated to pediatric airway management and ventilation.

- Improve data collection for integration into national and international databases.

- Increase the use of video or passive monitoring / data collection technology that can more accurately document timing and care processes.

- Conduct more research on waveform capnography to confirm successful ventilation with each type of airway and ventilation device.

- Conduct mechanistic studies in humans to advance understanding of underlying pathophysiologies by which differences may be occurring.

- Further assess the impact race and sex have on different airway management strategies. 


\section{Conclusion}

Overall, there is limited evidence to suggest differences in patient-oriented outcomes between use of bag valve mask (BVM), supraglottic airway (SGA), and endotracheal intubation (ETI) in the management of prehospital airway. The objective of this systematic review and meta-analysis was to identify and synthesize the available evidence to support the development of evidence-based recommendations and guidelines for prehospital airway management. From the beginning, all participants, contributors, and stakeholders involved in this process were aware that the outcome would not be a simple set of algorithmic protocols. This topic converges vast variation in multiple factors influencing prehospital airway management (patient characteristics, emergency types, provider level) in an emergent environment that defies control, thereby limiting the ability to systematically apply and study interventions. The findings are presented for clinically meaningful patient populations and are not summarized across groups because that would not be clinically appropriate. The findings from this effort are detailed and comprehensive and it is important to use them to inform policy, practice, education, and research to improve prehospital airway management and ventilation support to optimize patient outcomes. 


\section{References}

1. Adnet F, Borron SW, Racine SX, et al. The intubation difficulty scale (IDS): proposal and evaluation of a new score characterizing the complexity of endotracheal intubation. Anesthesiology. 1997 Dec;87(6):1290-7. doi: 10.1097/00000542-199712000-00005. PMID: 9416711.

2. Caruana E, Duchateau FX, Cornaglia C, et al. Tracheal intubation related complications in the prehospital setting. Emerg Med J. 2015 Nov;32(11):882-7. doi: 10.1136/emermed-2013-203372. PMID: 25604325 .

3. Cook T, Behringer EC, Benger J. Airway management outside the operating room: hazardous and incompletely studied. Curr Opin Anaesthesiol. 2012 Aug;25(4):461-9. doi: 10.1097/ACO.0b013e32835528b1. PMID: 22673785.

4. Garnier M, Bonnet F. Management of anesthetic emergencies and complications outside the operating room. Curr Opin Anaesthesiol. 2014 Aug;27(4):437-41. doi: 10.1097/ACO.0000000000000088. PMID: 24762955.

5. Pepe PE, Roppolo LP, Fowler RL. Prehospital endotracheal intubation: elemental or detrimental? Crit Care. 2015 Mar 16;19:121. doi: 10.1186/s13054-0150808-x. PMID: 25887350.

6. Brown JB, Kheng M, Carney NA, et al. Geographical Disparity and Traumatic Brain Injury in America: Rural Areas Suffer Poorer Outcomes. J Neurosci Rural Pract. 2019 Jan-Mar;10(1):10-5. doi: 10.4103/jnrp.jnrp_310_18. PMID: 30765964.

7. Agency for Healthcare Research and Quality. Agency for Healthcare Research and Quality, 2010, Future Research Needs Methods Research Series, https:/effectivehealthcare.ahrq.gov/products /future-research-needs-methods/overview. Accessed 12/10/2019.
8. Higgins J, Savović J, Page M, et al. Chapter 8: Assessing risk of bias in a randomized trial. In: Higgins J, Thomas J, Chandler J, Cumpston M, Li T, Page M, et al., eds. Cochrane Handbook for Systematic Reviews of Interventions version 6 (updated July 2019): Cochrane; 2019.

9. United States Preventive Services Task Force. US Preventive Services Task Force. US Preventive Services Task Force Procedure Manual. Rockville, MD: Agency for Healthcare Research and Quality: 2018. https://www.uspreventiveservicestaskforce.o rg/Page/Name/procedure-manual. Accessed December 26, 2019.

10. Hardy RJ, Thompson SG. A likelihood approach to meta-analysis with random effects. Stat Med. 1996 Mar 30;15(6):61929. doi: 10.1002/(sici)10970258(19960330)15:6<619::Aidsim188>3.0.Co;2-a. PMID: 8731004.

11. Zhang J, Yu KF. What's the relative risk? A method of correcting the odds ratio in cohort studies of common outcomes. JAMA. 1998;280(19):1690-1. doi: 10.1001/jama.280.19.1690.

12. Higgins JP, Thompson SG. Quantifying heterogeneity in a meta-analysis. Stat Med. 2002 Jun 15;21(11):1539-58. doi: 10.1002/sim.1186. PMID: 12111919.

13. Fiala A, Lederer W, Neumayr A, et al. EMT-led laryngeal tube vs. face-mask ventilation during cardiopulmonary resuscitation - a multicenter prospective randomized trial. Scand J Trauma Resusc Emerg Med. 2017 Oct 26;25(1):104. doi: 10.1186/s13049-017-0446-1. PMID: 29073915.

14. Lupton JR, Schmicker RH, Stephens S, et al. Outcomes with the use of bag-valve-mask ventilation during out-of-hospital cardiac arrest in the Pragmatic Airway Resuscitation Trial. Acad Emerg Med. 2020

May;27(5):366-74. doi: 10.1111/acem.13927. PMID: 32220129. 
15. Maignan M, Koch FX, Kraemer M, et al. Impact of laryngeal tube use on chest compression fraction during out-of-hospital cardiac arrest. A prospective alternate month study. Resuscitation. 2015 Aug;93:113-7. doi: 10.1016/j.resuscitation.2015.06.002. PMID: 26070831.

16. Rumball CJ, MacDonald D. The PTL, Combitube, laryngeal mask, and oral airway: a randomized prehospital comparative study of ventilatory device effectiveness and cost-effectiveness in 470 cases of cardiorespiratory arrest. Prehosp Emerg Care. 1997 Jan-Mar;1(1):1-10. doi: 10.1080/10903129708958776. PMID: 9709312 .

17. Wang HE, Schmicker RH, Daya MR, et al. Effect of a strategy of initial laryngeal tube insertion vs endotracheal intubation on 72 hour survival in adults with out-of-hospital cardiac arrest: a randomized clinical trial. JAMA. 2018 Aug 28;320(8):769-78. doi: 10.1001/jama.2018.7044. PMID: 30167699.

18. Hansen M, Wang H, Le N, et al. Prospective evaluation of airway management in pediatric out-of-hospital cardiac arrest. Resuscitation. 2020 Aug 11;11:11. doi: 10.1016/j.resuscitation.2020.08.003. PMID: 32795596 .

19. Roth D, Hafner C, Aufmesser W, et al. Safety and feasibility of the laryngeal tube when used by EMTs during out-of-hospital cardiac arrest. Am J Emerg Med. 2015

Aug;33(8):1050-5. doi: 10.1016/j.ajem.2015.04.048. PMID: 25957625 .

20. Sos-Kanto study group. Comparison of arterial blood gases of laryngeal mask airway and bag-valve-mask ventilation in out-of-hospital cardiac arrests. Circ J. 2009 Mar;73(3):490-6. doi: 10.1253/circj.cj-080874. PMID: 19194045.

21. Sulzgruber P, Datler P, Sterz F, et al. The impact of airway strategy on the patient outcome after out-of-hospital cardiac arrest: a propensity score matched analysis. Europ Heart J Acute Cardiovasc Care. 2018 Aug;7(5):423-31. doi: 10.1177/2048872617731894. PMID: 28948850.
22. Takei Y, Enami M, Yachida T, et al. Tracheal intubation by paramedics under limited indication criteria may improve the short-term outcome of out-of-hospital cardiac arrests with noncardiac origin. J Anesth. 2010 Oct;24(5):716-25. doi: 10.1007/s00540-010-0974-6. PMID: 20577765.

23. Chiang WC, Hsieh MJ, Chu HL, et al. The effect of successful intubation on patient outcomes after out-of-hospital cardiac arrest in taipei. Ann Emerg Med. 2018 Mar;71(3):387-96.e2. doi: 10.1016/j.annemergmed.2017.08.008. PMID: 28967516.

24. Evans CC, Petersen A, Meier EN, et al. Prehospital traumatic cardiac arrest: management and outcomes from the resuscitation outcomes consortium epistrytrauma and PROPHET registries. J Trauma Acute Care Surg. 2016 Aug;81(2):285-93. doi: 10.1097/TA.0000000000001070. PMID: 27070438.

25. Hanif MA, Kaji AH, Niemann JT. Advanced airway management does not improve outcome of out-of-hospital cardiac arrest. Acad Emerg Med. 2010 Sep;17(9):926-31. doi: 10.1111/j.15532712.2010.00829.x. PMID: 20836772.

26. Hansen ML, Lin A, Eriksson C, et al. A comparison of pediatric airway management techniques during out-of-hospital cardiac arrest using the CARES database.

Resuscitation. 2017 Nov; 120:51-6. doi: 10.1016/j.resuscitation.2017.08.015. PMID: 28838781 .

27. Hardy GB, Maddry JK, Ng PC, et al. Impact of prehospital airway management on combat mortality. Am J Emerg Med. 2018 Jun;36(6):1032-5. doi: 10.1016/j.ajem.2018.02.007. PMID: 29691106.

28. Kang K, Kim T, Ro YS, et al. Prehospital endotracheal intubation and survival after out-of-hospital cardiac arrest: results from the Korean nationwide registry. Am J Emerg Med. 2016 Feb;34(2):128-32. doi: 10.1016/j.ajem.2015.09.036. PMID: 26597496. 
29. Nagao T, Kinoshita K, Sakurai A, et al. Effects of bag-mask versus advanced airway ventilation for patients undergoing prolonged cardiopulmonary resuscitation in pre-hospital setting. J Emerg Med. 2012 Feb;42(2):162-70. doi: 10.1016/j.jemermed.2011.02.020. PMID: 22032811.

30. Noda E, Zaitsu A, Hashizume M, et al. Prognosis of patient with cardiopulmonary arrest transported to Kyushu University Hospital. Fukuoka Igaku Zasshi. 2007 Mar;98(3):73-81. PMID: 17461032.

31. Ohashi-Fukuda N, Fukuda T, Yahagi N. Effect of pre-hospital advanced airway management for out-of-hospital cardiac arrest caused by respiratory disease: a propensity score-matched study. Anaesth Intensive Care. 2017 May;45(3):375-83. doi: 10.1177/0310057X1704500314. PMID: 28486897.

32. Shin SD, Ahn KO, Song KJ, et al. Out-ofhospital airway management and cardiac arrest outcomes: a propensity score matched analysis. Resuscitation. 2012 Mar;83(3):313-9. doi: 10.1016/j.resuscitation.2011.10.028. PMID: 22101202 .

33. Yanagawa Y, Sakamoto T. Analysis of prehospital care for cardiac arrest in an urban setting in Japan. J Emerg Med. 2010 Apr;38(3):340-5. doi:

10.1016/j.jemermed.2008.04.037. PMID: 18993021 .

34. Chien LC, Hsu HC, Lin CH, et al. Use of an intubating laryngeal mask airway on out-ofhospital cardiac arrest patients in a developing emergency medical service system. J Formos Med Assoc. 2012 Jan;111(1):24-9. doi: 10.1016/j.jfma.2012.01.004. PMID: 22333009 .

35. Gausche M, Lewis RJ, Stratton SJ, et al. Effect of out-of-hospital pediatric endotracheal intubation on survival and neurological outcome: a controlled clinical trial. see comment erratum appears in JAMA 2000 Jun 28;283(24): 3204. JAMA. 2000;6:783-90. doi:

10.1001/jama.283.6.783. PMID: 10683058.
36. Gausche-Hill M, Lewis RJ, Gunter CS, et al. Design and implementation of a controlled trial of pediatric endotracheal intubation in the out-of-hospital setting. Ann Emerg Med. 2000 Oct;36(4):356-65. doi: 10.1067/mem.2000.109447. PMID: 11020685 .

37. Jabre P, Penaloza A, Pinero D, et al. Effect of bag-mask ventilation vs endotracheal intubation during cardiopulmonary resuscitation on neurological outcome after out-of-hospital cardiorespiratory arrest: a randomized clinical trial. JAMA. $2018 \mathrm{Feb}$ 27;319(8):779-87. doi: 10.1001/jama.2018.0156. PMID: 29486039.

38. Malinverni S, Bartiaux M, Cavallotto F, et al. Does endotracheal intubation increases chest compression fraction in out of hospital cardiac arrest: a substudy of the CAAM trial. Resuscitation. 2019 Apr;137:35-40. doi: 10.1016/j.resuscitation.2019.01.032. PMID: 30753851.

39. Bernard SA, Nguyen V, Cameron P, et al. Prehospital rapid sequence intubation improves functional outcome for patients with severe traumatic brain injury: a randomized controlled trial. Ann Surg. 2010 Dec;252(6):959-65. doi: 10.1097/SLA.0b013e3181efc15f. PMID: 21107105.

40. Cooper A, DiScala C, Foltin G, et al. Prehospital endotracheal intubation for severe head injury in children: a reappraisal. Semin Pediatr Surg. 2001 Feb;10(1):3-6. doi: 10.1053/spsu.2001.19379. PMID: 11172563.

41. Eckstein M, Chan L, Schneir A, et al. Effect of prehospital advanced life support on outcomes of major trauma patients. J Trauma. 2000 Apr;48(4):643-8. doi: 10.1097/00005373-200004000-00010. PMID: 10780596.

42. Stockinger ZT, McSwain NE, Jr. Prehospital endotracheal intubation for trauma does not improve survival over bag-valve-mask ventilation. J Trauma. 2004 Mar;56(3):5316. doi: 10.1097/01.ta.0000111755.94642.29. PMID: 15128123. 
43. Yuksen C, Phattharapornjaroen P, Kreethep $\mathrm{W}$, et al. Bag-valve mask versus endotracheal intubation in out-of-hospital cardiac arrest on return of spontaneous circulation: a national database study. Open Access Emerg Med. 2020;12:43-6. doi: 10.2147/OAEM.S229356. PMID: 32210644.

44. Benger JR, Kirby K, Black S, et al. Effect of a strategy of a supraglottic airway device vs tracheal intubation during out-of-hospital cardiac arrest on functional outcome: the AIRWAYS-2 randomized clinical trial. JAMA. 2018 Aug 28;320(8):779-91. doi: 10.1001/jama.2018.11597. PMID: 30167701 .

45. Frascone RJ, Russi C, Lick C, et al. Comparison of prehospital insertion success rates and time to insertion between standard endotracheal intubation and a supraglottic airway. Resuscitation. 2011 Dec;82(12):1529-36. doi: 10.1016/j.resuscitation.2011.07.009. PMID: 21763247.

46. Rabitsch W, Schellongowski P, Staudinger $\mathrm{T}$, et al. Comparison of a conventional tracheal airway with the Combitube in an urban emergency medical services system run by physicians. Resuscitation. 2003 Apr;57(1):27-32. doi: 10.1016/s03009572(02)00435-5. PMID: 12668296.

47. Bartlett RL, Martin SD, McMahon JM, Jr., et al. A field comparison of the pharyngeotracheal lumen airway and the endotracheal tube. J Trauma. 1992 Mar;32(3):280-4. doi: 10.1097/00005373199203000-00002. PMID: 1548713.

48. Rumball C, Macdonald D, Barber P, et al. Endotracheal intubation and esophageal tracheal Combitube insertion by regular ambulance attendants: a comparative trial. Prehosp Emerg Care. 2004 Jan-Mar;8(1):1522. doi: 10.1080/312703002764. PMID: 14691782.
49. Hankins DG, Carruthers N, Frascone RJ, et al. Complication rates for the esophageal obturator airway and endotracheal tube in the prehospital setting. Prehospital Disaster Med. 1993 Apr-Jun;8(2):117-21. doi: 10.1017/s1049023x00040176. PMID: 10148602.

50. Hiltunen P, Jantti H, Silfvast T, et al. Airway management in out-of-hospital cardiac arrest in Finland: current practices and outcomes. Scand J Trauma Resusc Emerg Med. 2016 Apr 12;24:49. doi: 10.1186/s13049-016-0235-2. PMID: 27071823.

51. Jarman AF, Hopkins CL, Hansen JN, et al. Advanced airway type and its association with chest compression interruptions during out-of-hospital cardiac arrest resuscitation attempts. Prehosp Emerg Care. 2017 SepOct;21(5):628-35. doi: 10.1080/10903127.2017.1308611. PMID: 28459305.

52. Kajino K, Iwami T, Kitamura T, et al. Comparison of supraglottic airway versus endotracheal intubation for the pre-hospital treatment of out-of-hospital cardiac arrest. Crit Care. 2011;15(5):R236. doi: 10.1186/cc10483. PMID: 21985431.

53. McCall MJ, Reeves M, Skinner M, et al. Paramedic tracheal intubation using the intubating laryngeal mask airway. Prehosp Emerg Care. 2008 Jan-Mar;12(1):30-4. doi: 10.1080/10903120701709803. PMID: 18189174.

54. McMahan S, Ornato JP, Racht EM, et al. Multi-agency, prehospital evaluation of the pharyngeo-tracheal lumen (PTL) airway. Prehospital Disaster Med. 1992 JanMar;7(1):13-8. doi: 10.1017/s1049023x00039145. PMID: 10149691.

55. Becker TK, Berning AW, Prabhu A, et al. An assessment of ventilation and perfusion markers in out-of-hospital cardiac arrest patients receiving mechanical CPR with endotracheal or supraglottic airways. Resuscitation. 2018 Jan;122:61-4. doi: 10.1016/j.resuscitation.2017.11.054. PMID: 29175355. 
56. Cady CE, Pirrallo RG. The effect of Combitube use on paramedic experience in endotracheal intubation. Am J Emerg Med. 2005 Nov;23(7):868-71. doi: 10.1016/j.ajem.2005.07.013. PMID: 16291443.

57. Davis DP, Peay J, Sise MJ, et al. The impact of prehospital endotracheal intubation on outcome in moderate to severe traumatic brain injury. J Trauma. 2005;58(5):933-9. doi: 10.1097/01.TA.0000162731.53812.58. PMID: 15920406.

58. Duckett J, Fell P, Han K, et al. Introduction of the I-gel supraglottic airway device for prehospital airway management in a UK ambulance service. Emerg Med J. 2014 Jun;31(6):505-7. doi: 10.1136/emermed2012-202126. PMID: 23576232.

59. Edwards T, Williams J, Cottee M. Influence of prehospital airway management on neurological outcome in patients transferred to a heart attack centre following out-ofhospital cardiac arrest. Emerg Med Australas. 2019 Feb;31(1):76-82. doi: 10.1111/1742-6723.13107. PMID: 29752776.

60. Gamberini L, Giugni A, Ranieri S, et al. Early-onset ventilator-associated pneumonia in severe traumatic brain injury: is there a relationship with prehospital airway management? J Emerg Med. 2019 Jun;56(6):657-65. doi: 10.1016/j.jemermed.2019.02.005. PMID: 31000428 .

61. Jarvis JL, Wampler D, Wang HE. Association of patient age with first pass success in out-of-hospital advanced airway management. Resuscitation. 2019

Aug;141:136-43. doi:

10.1016/j.resuscitation.2019.06.002. PMID: 31238034 .

62. McMullan J, Gerecht R, Bonomo J, et al. Airway management and out-of-hospital cardiac arrest outcome in the CARES registry. Resuscitation. 2014

May;85(5):617-22. doi:

10.1016/j.resuscitation.2014.02.007. PMID: 24561079 .
63. Steuerwald MT, Braude DA, Petersen TR, et al. Preliminary report: comparing aspiration rates between prehospital patients managed with extraglottic airway devices and endotracheal intubation. Air Med J. 2018 Jul - Aug;37(4):240-3. doi: 10.1016/j.amj.2018.04.004. PMID: 29935702.

64. Tanabe S, Ogawa T, Akahane M, et al. Comparison of neurological outcome between tracheal intubation and supraglottic airway device insertion of out-of-hospital cardiac arrest patients: a nationwide, population-based, observational study. J Emerg Med. 2013 Feb;44(2):389-97. doi: 10.1016/j.jemermed.2012.02.026. PMID: 22541878 .

65. Behrens NH, Fischer M, Krieger T, et al. Effect of airway management strategies during resuscitation from out-of-hospital cardiac arrest on clinical outcome: a registry-based analysis. Resuscitation. 2020 Jul 152:157-64. doi: 10.1016/j.resuscitation.2020.04.015. PMID: 32387124 .

66. Fukuda T, Sekiguchi H, Taira T, et al. Type of advanced airway and survival after pediatric out-of-hospital cardiac arrest. Resuscitation. 2020 May;150:145-53. doi: 10.1016/j.resuscitation.2020.02.005. PMID: 32070779 .

67. Nwanne T, Jarvis J, Barton D, et al. Advanced airway management success rates in a national cohort of emergency medical services agencies. Resuscitation. 2020 Jan 01;146:43-9. doi: 10.1016/j.resuscitation.2019.11.006. PMID: 31756361 .

68. Wang HE, Szydlo D, Stouffer JA, et al. Endotracheal intubation versus supraglottic airway insertion in out-of-hospital cardiac arrest. Resuscitation. 2012 Sep;83(9):10616. doi: 10.1016/j.resuscitation.2012.05.018. PMID: 22664746. 
69. Gahan K, Studnek JR, Vandeventer S. King LT-D use by urban basic life support first responders as the primary airway device for out-of-hospital cardiac arrest. Resuscitation. 2011 Dec;82(12):1525-8. doi:

10.1016/j.resuscitation.2011.06.036. PMID: 21756859 .

70. Bendinelli C, Ku D, Nebauer S, et al. A tale of two cities: prehospital intubation with or without paralysing agents for traumatic brain injury. ANZ J Surg. 2018 May;88(5):455-9. doi: 10.1111/ans.14479. PMID: 29573111.

71. Bozeman WP, Kleiner DM, Huggett V. A comparison of rapid-sequence intubation and etomidate-only intubation in the prehospital air medical setting. Prehosp Emerg Care. 2006 Jan-Mar;10(1):8-13. doi: 10.1080/10903120500366854. PMID: 16418085 .

72. Bulger EM, Copass MK, Sabath DR, et al. The use of neuromuscular blocking agents to facilitate prehospital intubation does not impair outcome after traumatic brain injury. J Trauma. 2005 Apr;58(4):718-23; discussion 23-4. doi: 10.1097/01.ta.0000159239.14181.bc. PMID: 15824647.

73. Cudnik MT, Newgard CD, Daya M, et al. The impact of rapid sequence intubation on trauma patient mortality in attempted prehospital intubation. J Emerg Med. 2010 Feb;38(2):175-81. doi:

10.1016/j.jemermed.2008.01.022. PMID: 18790586.

74. Eberlein CM, Luther IS, Carpenter TA, et al. First-pass success intubations using video laryngoscopy versus direct laryngoscopy: a retrospective prehospital ambulance service study. Air Med J. 2019 Sep - Oct;38(5):3568. doi: 10.1016/j.amj.2019.06.004. PMID: 31578974 .

75. Fouche PF, Smith K, Jennings PA, et al. The association of paramedic rapid sequence intubation and survival in out-of-hospital stroke. Emerg Med J. 2019 Jul;36(7):41622. doi: 10.1136/emermed-2019-208613. PMID: 31147349.
76. Hoffmann M, Czorlich P, Lehmann W, et al. The impact of prehospital intubation with and without sedation on outcome in trauma patients with a GCS of 8 or less. $J$

Neurosurg Anesthesiol. 2017

Apr;29(2):161-7. doi: 10.1097/ANA.0000000000000275. PMID: 26797107.

77. Kwok H, Prekker M, Grabinsky A, et al. Use of rapid sequence intubation predicts improved survival among patients intubated after out-of-hospital cardiac arrest. Resuscitation. 2013 Oct;84(10):1353-8. doi: 10.1016/j.resuscitation.2013.04.015. PMID: 23665389.

78. Myers LA, Gallet CG, Kolb LJ, et al. Determinants of success and failure in prehospital endotracheal intubation. West J Emerg Med. 2016 Sep;17(5):640-7. doi: 10.5811/westjem.2016.6.29969. PMID: 27625734.

79. Powell EK, Hinckley WR, Stolz U, et al. Predictors of definitive airway sans hypoxia/hypotension on first attempt (DASH-1A) success in traumatically injured patients undergoing prehospital intubation. Prehosp Emerg Care. 2019 Oct 07:1-8. doi: 10.1080/10903127.2019.1670299. PMID: 31539287.

80. Prekker ME, Kwok H, Shin J, et al. The process of prehospital airway management: challenges and solutions during paramedic endotracheal intubation. Crit Care Med. 2014 Jun;42(6):1372-8. doi: 10.1097/CCM.0000000000000213. PMID: 24589641 .

81. Sobuwa S, Hartzenberg HB, Geduld H, et al. Outcomes following prehospital airway management in severe traumatic brain injury. S Afr Med J. 2013 Jul 29;103(9):644-6. doi: 10.7196/samj.7035. PMID: 24300684.

82. Sunde GA, Heltne JK, Lockey D, et al. Airway management by physician-staffed helicopter emergency medical services - a prospective, multicentre, observational study of 2,327 patients. Scand J Trauma Resusc Emerg Med. 2015 Aug 07;23:57. doi: 10.1186/s13049-015-0136-9. PMID: 26250700 . 
83. Vilke GM, Hoyt DB, Epperson M, et al. Intubation techniques in the helicopter. J Emerg Med. 1994 Mar-Apr;12(2):217-24. doi: 10.1016/0736-4679(94)90702-1. PMID: 8207159 .

84. Wang HE, Yealy DM. How many attempts are required to accomplish out-of-hospital endotracheal intubation? Acad Emerg Med. 2006 Apr;13(4):372-7. doi:

10.1197/j.aem.2005.11.001. PMID: 16531595 .

85. Arima T, Nagata O, Miura T, et al. Comparative analysis of airway scope and Macintosh laryngoscope for intubation primarily for cardiac arrest in prehospital setting. Am J Emerg Med. 2014 Jan;32(1):40-3. doi: 10.1016/j.ajem.2013.09.026. PMID: 24176585 .

86. Breeman W, Van Vledder MG, Verhofstad MHJ, et al. First attempt success of video versus direct laryngoscopy for endotracheal intubation by ambulance nurses: a prospective observational study. Eur J Trauma Emerg Surg. 2020 Feb 19;19:19. doi: 10.1007/s00068-020-01326-z. PMID: 32072225 .

87. Ducharme S, Kramer B, Gelbart D, et al. A pilot, prospective, randomized trial of video versus direct laryngoscopy for paramedic endotracheal intubation. Resuscitation. 2017 May;114:121-6. doi:

10.1016/j.resuscitation.2017.03.022. PMID: 28336412 .

88. Jarvis JL, McClure SF, Johns D. EMS intubation improves with king vision video laryngoscopy. Prehosp Emerg Care. 2015;19(4):482-9. doi: 10.3109/10903127.2015.1005259. PMID: 25909850 .

89. Kreutziger J, Hornung S, Harrer C, et al. Comparing the megrath mac video laryngoscope and direct laryngoscopy for prehospital emergency intubation in air rescue patients: a multicenter, randomized, controlled trial. Crit Care Med. 2019 Oct;47(10):1362-70. doi: 10.1097/CCM.0000000000003918. PMID: 31389835 .
90. Louka A, Stevenson C, Jones G, et al. Intubation success after introduction of a quality assurance program using video laryngoscopy. Air Med J. 2018 Sep;37(5):303-5. doi: 10.1016/j.amj.2018.05.001. PMID: 30322632 .

91. Macke C, Gralla F, Winkelmann M, et al. Increased first pass success with C-MAC videolaryngoscopy in prehospital endotracheal intubation-a randomized controlled trial. J Clin Med. 2020 Aug 22;9(9):22. doi: 10.3390/jcm9092719. PMID: 32842705.

92. Olvera DJ, Stuhlmiller DFE, Wolfe A, et al. A continuous quality improvement airway program results in sustained increases in intubation success. Prehosp Emerg Care. 2018 Sep-Oct;22(5):602-7. doi: 10.1080/10903127.2018.1433734. PMID: 29465279.

93. Risse J, Volberg C, Kratz T, et al. Comparison of videolaryngoscopy and direct laryngoscopy by German paramedics during out-of-hospital cardiopulmonary resuscitation; an observational prospective study. BMC Emerg Med. 2020 Mar 23;20(1):22. doi: 10.1186/s12873-02000316-z. PMID: 32293276.

94. Trimmel H, Kreutziger J, Fertsak G, et al. Use of the Airtraq laryngoscope for emergency intubation in the prehospital setting: a randomized control trial. Crit Care Med. 2011 Mar;39(3):489-93. doi: 10.1097/CCM.0b013e318206b69b. PMID: 21169822.

95. Trimmel H, Kreutziger J, Fitzka R, et al. Use of the glidescope ranger video laryngoscope for emergency intubation in the prehospital setting: a randomized control trial. Crit Care Med. 2016 Jul;44(7):e470-6. doi: 10.1097/CCM.0000000000001669. PMID: 27002277.

96. Wayne MA, McDonnell M. Comparison of traditional versus video laryngoscopy in outof-hospital tracheal intubation. Prehosp Emerg Care. 2010 Apr-Jun;14(2):278-82. doi: 10.3109/10903120903537189. PMID: 20199237. 
97. Jabre P, Galinski M, Ricard-Hibon A, et al. Out-of-hospital tracheal intubation with single-use versus reusable metal laryngoscope blades: a multicenter randomized controlled trial. Ann Emerg Med. 2011 Mar;57(3):225-31. doi: 10.1016/j.annemergmed.2010.10.011. PMID: 21129822.

98. Dos Santos FD, Schnakofsky R, Cascio A, et al. Disposable stainless steel vs plastic laryngoscope blades among paramedics. Am J Emerg Med. 2011 Jul;29(6):590-3. doi: 10.1016/j.ajem.2009.12.022. PMID: 20825833.

99. Jabre P, Leroux B, Brohon S, et al. A comparison of plastic single-use with metallic reusable laryngoscope blades for out-of-hospital tracheal intubation. Ann Emerg Med. 2007 Sep;50(3):258-63. doi: 10.1016/j.annemergmed.2007.04.022. PMID: 17583382.

100. Chan M, Fehlmann CA, Pasquier M, et al. Endotracheal intubation success rate in an urban, supervised, resident-staffed emergency mobile system: an 11-year retrospective cohort study. J Clin Med. 2020b Jan 16;9(1):16. doi: 10.3390/jcm9010238. PMID: 31963162.

101. Delorenzo A, St Clair T, Andrew E, et al. Prehospital rapid sequence intubation by intensive care flight paramedics. Prehosp Emerg Care. 2018 Sep-Oct;22(5):595-601. doi: 10.1080/10903127.2018.1426666. PMID: 29405803.

102. Gellerfors M, Larsson A, Svensen CH, et al. Use of the Airtraq device for airway management in the prehospital setting--a retrospective study. Scand J Trauma Resusc Emerg Med. 2014 Feb 03;22:10. doi: 10.1186/1757-7241-22-10. PMID: 24484856 .

103. Hossfeld B, Thierbach S, Allgoewer A, et al. First pass success of tracheal intubation using the C-MAC PM videolaryngoscope as first-line device in prehospital cardiac arrest compared with other emergencies: an observational study. Eur J Anaesthesiol. 2020 Aug 21;21:21. doi: 10.1097/EJA.0000000000001286. PMID: 32833853 .
104. Rocca B, Crosby E, Maloney J, et al. An assessment of paramedic performance during invasive airway management. Prehosp Emerg Care. 2000 AprJun;4(2):164-7. doi: 10.1080/10903120090941443. PMID: 10782606.

105. Eich C, Roessler M, Nemeth M, et al. Characteristics and outcome of prehospital paediatric tracheal intubation attended by anaesthesia-trained emergency physicians. Resuscitation. 2009 Dec;80(12):1371-7. doi: 10.1016/j.resuscitation.2009.09.004. PMID: 19804939.

106. Prekker ME, Delgado F, Shin J, et al. Pediatric intubation by paramedics in a large emergency medical services system: process, challenges, and outcomes. Ann Emerg Med. 2016 Jan;67(1):20-9.e4. doi: 10.1016/j.annemergmed.2015.07.021. PMID: 26320522.

107. Garza AG, Algren DA, Gratton MC, et al. Populations at risk for intubation nonattempt and failure in the prehospital setting. Prehosp Emerg Care. 2005 AprJun;9(2):163-6. doi: 10.1080/10903120590924654. PMID: 16036840 .

108. Murray JA, Demetriades D, Berne TV, et al. Prehospital intubation in patients with severe head injury. J Trauma. 2000 Dec;49(6):1065-70. doi: 10.1097/00005373200012000-00015. PMID: 11130490.

109. Studnek JR, Thestrup L, Vandeventer S, et al. The association between prehospital endotracheal intubation attempts and survival to hospital discharge among out-ofhospital cardiac arrest patients. Acad Emerg Med. 2010 Sep;17(9):918-25. doi: 10.1111/j.1553-2712.2010.00827.x. PMID: 20836771.

110. Benger J, Coates D, Davies S, et al. Randomised comparison of the effectiveness of the laryngeal mask airway supreme, i-gel and current practice in the initial airway management of out of hospital cardiac arrest: a feasibility study. Br J Anaesth. 2016 Feb;116(2):262-8. doi: 10.1093/bja/aev477. PMID: 26787796. 
111. Middleton PM, Simpson PM, Thomas RE, et al. Higher insertion success with the i-gel supraglottic airway in out-of-hospital cardiac arrest: a randomised controlled trial. Resuscitation. 2014 Jul;85(7):893-7. doi: 10.1016/j.resuscitation.2014.02.021. PMID: 24594090 .

112. Ono Y, Hayakawa M, Maekawa K, et al. Should laryngeal tubes or masks be used for out-of-hospital cardiac arrest patients? Am J Emerg Med. 2015 Oct;33(10):1360-3. doi: 10.1016/j.ajem.2015.07.043. PMID: 26306437.

113. Chan JJ, Goh ZX, Koh ZX, et al. Clinical evaluation of the use of laryngeal tube verses laryngeal mask airway for out-ofhospital cardiac arrest by paramedics in Singapore. Singapore Med J. 2020a Aug 17;17:17. doi: 10.11622/smedj.2020119. PMID: 32798357.

114. Andersen LW, Grossestreuer AV, Donnino MW. "Resuscitation time bias"-A unique challenge for observational cardiac arrest research. Resuscitation. 2018;125:79-82. doi: 10.1016/j.resuscitation.2018.02.006. PMID: 29425975.

115. Aufderheide TP, Lurie KG. Death by hyperventilation: a common and lifethreatening problem during cardiopulmonary resuscitation. Crit Care Med. 2004 Sep;32(9 Suppl):S345-51. doi: 10.1097/01.ccm.0000134335.46859.09. PMID: 15508657.

116. Aufderheide TP, Sigurdsson G, Pirrallo RG, et al. Hyperventilation-induced hypotension during cardiopulmonary resuscitation. Circulation. 2004 Apr 27;109(16):1960-5. doi: 10.1161/01.Cir.0000126594.79136.61. PMID: 15066941.

117. Davis DP, Dunford JV, Poste JC, et al. The impact of hypoxia and hyperventilation on outcome after paramedic rapid sequence intubation of severely head-injured patients. J Trauma. 2004 Jul;57(1):1-8; discussion 10. doi: 10.1097/01.ta.0000135503.71684.c8. PMID: 15284540 .
118. Gaither JB, Spaite DW, Bobrow BJ, et al. Balancing the potential risks and benefits of out-of-hospital intubation in traumatic brain injury: the intubation/hyperventilation effect. Ann Emerg Med. 2012 Dec;60(6):732-6. doi: 10.1016/j.annemergmed.2012.06.017. PMID: 22841182.

119. Pepe PE, Lurie KG, Wigginton JG, et al. Detrimental hemodynamic effects of assisted ventilation in hemorrhagic states. Crit Care Med. 2004 Sep;32(9 Suppl):S41420. doi:

10.1097/01.ccm.0000134264.88332.37. PMID: 15508670.

120. Elmer J, Torres C, Aufderheide TP, et al. Association of early withdrawal of lifesustaining therapy for perceived neurological prognosis with mortality after cardiac arrest. Resuscitation. 2016;102:12735. doi: 10.1016/j.resuscitation.2016.01.016. PMID: 26836944.

121. del Junco DJ, Fox EE, Camp EA, et al. Seven deadly sins in trauma outcomes research: an epidemiologic post mortem for major causes of bias. J Trauma Acute Care Surg. 2013;75(1 Suppl 1):S97-S103. doi: 10.1097/TA.0b013e318298b0a4. PMID: 23778519.

122. Methods Guide for Effectiveness and Comparative Effectiveness Reviews. AHRQ Publication No. 10 (14)-EHC063-EF. Rockville, MD: Agency for Healthcare Research and Quality; January 2014. www.effectivehealthcare.ahrq.gov.

123. Lau J, Chang S, Berkman N, et al. EPC Response to IOM Standards for Systematic Reviews. Rockville, MD: Agency for Healthcare Research and Quality; 2013. https://www.ncbi.nlm.nih.gov/books/NBK1 37838/.

124. Lupton JR, Schmicker RH, Aufderheide TP, et al. Racial disparities in out-of-hospital cardiac arrest interventions and survival in the Pragmatic Airway Resuscitation Trial. Resuscitation. 2020 2020/08/11/doi: 10.1016/j.resuscitation.2020.08.004. 


\section{Abbreviations and Acronyms}

\begin{tabular}{|c|c|}
\hline Abbreviation & Definition \\
\hline $\mathrm{AHRQ}$ & Agency for Healthcare Research and Quality \\
\hline BiPAP & Bi-level positive airway pressure \\
\hline BVM & bag valve mask \\
\hline CCT & controlled clinical trial \\
\hline CPAP & continuous positive airway pressure \\
\hline CPC Score & Cerebral Performance Category Score \\
\hline DASH-1A & Definitive Airway Sans Hypoxia/Hypotension on First Attempt \\
\hline DSI & delayed sequence intubation \\
\hline ED & emergency department \\
\hline EMS & emergency medical services \\
\hline EPC & Evidence-based Practice Center \\
\hline ETI & endotracheal intubation \\
\hline GOS & Glasgow Outcome Scale \\
\hline ICU & intensive care unit \\
\hline ITT & intent to treat \\
\hline $\mathrm{KQ}$ & Key Question \\
\hline LMA & laryngeal mask airway \\
\hline LT & Laryngeal tube \\
\hline $\mathrm{mRS}$ & modified Rankin Scale \\
\hline OBS & observational \\
\hline PICOS & population, intervention, comparator, outcome, setting, study design \\
\hline PTLA & Pharyngeotracheal lumen airway \\
\hline $\mathrm{RCT}$ & randomized controlled trial \\
\hline ROB & risk of bias \\
\hline ROSC & return of spontaneous circulation \\
\hline RSI & rapid sequence intubation \\
\hline SGA & supraglottic airway \\
\hline SOE & strength of evidence \\
\hline TEP & Technical Expert Panel \\
\hline TOO & Task Order Officer \\
\hline
\end{tabular}




\section{Appendix A. Methods}

\section{Details of Study Selection}

Publication date range. Studies were included that were published from January 1990 to September 2020. The beginning of the date range was selected based on the recommendation of the Key Informants and Technical Expert panels. Electronic searches will be updated to identify new publications while the draft report is subject to public and peer review. Literature identified during the updated search will be assessed following the same process of dual review as other studies considered for inclusion in the report.

Literature databases. MEDLINE ${ }^{\circledR}$, CINAHL $^{\circledR}$, the Cochrane Central Register of Controlled Trials, the Cochrane Database of Systematic Reviews, and Scopus ${ }^{\circledR}$ were searched to capture published literature.

Supplementing searches. A Supplemental Evidence and Data for Systematic review (SEADS) portal was available to facilitate submission of published and unpublished studies. Notice was posted in the Federal Register requesting published and unpublished evidence relevant to the review; no relevant submissions were received.

Hand searching. Reference lists of systematic reviews and included articles were reviewed to identify additional literature for inclusion.

The search was developed and executed by a research librarian with extensive systematic review experience and peer reviewed by a second librarian. The search strategies for each citation database are included below.

\section{Search Strategies}

Database: Ovid MEDLINE(R) ALL 1946 to September, 2020

1. exp emergency medical services/ or exp "transportation of patients"/ or triage/

2. ("emt" or "ems" or "emergency medical" or field or "paramedic*" or "prehospital" or "prehospital" or transport* or trauma or traumatic).ti,ab,kf.

3. 1 or 2

4. exp Airway Management/

5. (intubate or intubation or airway or ventilation or ventilatory).ti,ab,kf.

6. (endotracheal or supraglottic or tracheal or prehospital or "pre-hospital" or field).ti,ab,kf.

7. 5 and 6

8. "bag valve mask".ti,ab,kf.

9. (airway adj5 manage*).ti,ab,kf.

10. 4 or 7 or 8 or 9

11.3 and 10

12. limit 11 to $\mathrm{yr}=" 1990-2020 "$

13. (random or control or trial or cohort or case* or prospective or retrospective).ti,ab,kf,tw.

14. 12 and 13

15. exp cohort studies/

16. cohort\$.tw.

17. controlled clinical trial.pt.

18. exp case-control studies/

19. (case\$ and control\$).tw.

20. or/15-19 
21. randomized controlled trial.pt.

22. (random* or placebo* or control* or trial or blind*).ti,ab.

23. (animals not humans).sh.

24. (comment or editorial or meta-analysis or practice-guideline or review or letter).pt.

25. (21 or 22$)$ not (23 or 24$)$

26. 20 or 25

27. 12 and 26

28. 14 or 27

29. limit 28 to english language

30. "prehospital emergency care".jn.

31. "prehospital \& disaster medicine".jn.

32. "resuscitation".jn.

33. "military medicine".jn.

34. or $/ 30-33$

35. 10 and 34

36. limit 35 to $\mathrm{yr}=" 1990-2020 "$

37.29 or 36

Database: EBM Reviews - Cochrane Central Register of Controlled Trials $<$ September 2020> Search Strategy:

1 exp emergency medical services/ or exp "transportation of patients"/ or triage/

2 ("emt" or "ems" or "emergency medical" or field or "paramedic*" or "prehospital" or

"pre- hospital" or transport* or trauma or traumatic).ti,ab,hw.

31 or 2

$4 \exp$ Airway Management/

5 (intubate or intubation or airway or ventilation or ventilatory).ti,ab,hw.

6 (endotracheal or supraglottic or tracheal or prehospital or "pre-hospital" or field).ti,ab,hw.

$7 \quad 5$ and 6

8 "bag valve mask".ti,ab,hw.

9 (airway adj5 manage*).ti,ab,hw.

104 or 7 or 8 or 9

113 and 10

12 limit 11 to $\mathrm{yr}=" 1990-2020 "$

13 conference abstract.pt.

14 "journal: conference abstract".pt.

15 "journal: conference review".pt.

16 "http://.www.who.int/trialsearch*".so.

17 "https://clinicaltrials.gov*".so.

1813 or 14 or 15 or 16 or 17

1911 not 18

20 limit 19 to medline records

2119 not 20

Database: EBM Reviews - Cochrane Database of Systematic Reviews $<2005$ to September 8, 2020> 
Search Strategy:

1 ("emt" or "ems" or "emergency medical" or field or "paramedic*" or "prehospital" or "pre-hospital" or transport* or trauma or traumatic).ti,ab.

2 (intubate or intubation or airway or ventilation or ventilatory).ti,ab.

3 (endotracheal or supraglottic or tracheal or prehospital or "pre-hospital" or field).ti,ab.

$4 \quad 2$ and 3

5 "bag valve mask".ti,ab.

6 (airway adj5 manage*).ti,ab.

74 or 5 or 6

$8 \quad 1$ and 7

Database: EBSCOHost CINAHL PLUS September 8, 2020

S1 "emt" or "ems" or "emergency medical" or field or "paramedic*" or "prehospital" or " pre- hospital" or transport* or trauma or traumatic

S2 intubate or intubation or airway or ventilation or ventilatory

S3 endotracheal or supraglottic or tracheal or prehospital or "pre-hospital" or field

S4 bag valve mask

S5 "airway management"

S6 (MH "Airway Management+")

S7 (MH "Prehospital Care")

S8 S6 AND S7

S9 S2 AND S3

S10 S4 OR S9

S11 S1 AND S10

S12 S8 OR S11

S13 (MH "Experimental Studies+") OR (MH "Retrospective Design")

S14 random* or control* or trial or cohort or case* or prospective or retrospective

S15 S13 OR S14

S16 S12 AND S15

S17 Limiters - Published Date: 19900101-20201231; English Language; Exclude MEDLINE records

Database: Elsevier Scopus September 8, 2020

PUBYEAR > 1990 ( ( TITLE ( "emt" OR "ems" OR "emergency medical" OR field OR "paramedic*" OR "prehospital" OR "pre-hospital" OR transport* OR trauma OR traumatic )) AND ( ( ( TITLE ( intubate OR intubation OR airway OR ventilation OR ventilatory) AND TITLE (endotracheal OR supraglottic OR tracheal OR prehospital OR "pre-hospital" OR field )) ) OR ( TITLE ( "bag valve mask" OR "airway management" )) ) ) AND ( TITLE ( random* OR control OR trial OR cohort OR case* OR prospective OR retrospective ) )

\section{Inclusion and Exclusion Criteria}

The criteria for inclusion and exclusion of studies are based on the Key Questions and organized using the PICOS framework below (Table A-1). 
Table A-1. PICOS

\begin{tabular}{|c|c|c|}
\hline PICOS & Inclusion Criteria & Exclusion Criteria \\
\hline Populations & $\begin{array}{l}\text { Patients requiring prehospital ventilatory support or airway } \\
\text { protection who are treated in the prehospital setting by } \\
\text { emergency medical services personnel (paramedic, } \\
\text { advanced emergency medical technician, emergency } \\
\text { medical technician, emergency medical responder, etc.) }\end{array}$ & $\begin{array}{l}\text { - Patients treated with naloxone to } \\
\text { reverse opioid-related respiratory } \\
\text { failure } \\
\text { - Patients cared for in other than } \\
\text { the prehospital setting }\end{array}$ \\
\hline Interventions & $\begin{array}{l}\text { - Bag valve mask ventilation } \\
\text { - Supraglottic airway insertion, including dual-Iumen } \\
\text { airways } \\
\text { - Endotracheal intubation } \\
\text { ○ Via direct laryngoscopy with or without RSI or DSI } \\
\text { ○ Via video laryngoscopy with or without RSI or DSI }\end{array}$ & $\begin{array}{l}\text { - Nasotracheal intubation } \\
\text { - Percutaneous devices } \\
\text { - Surgical airway procedures } \\
\text { - CPAP and BiPAP }\end{array}$ \\
\hline Comparators & $\begin{array}{l}\text { KQ1: bag valve mask vs. supraglottic airway } \\
\text { KQ2: bag valve mask vs. endotracheal intubation } \\
\text { KQ3: supraglottic airway vs. endotracheal intubation } \\
\text { KQ4: different techniques for any one of the three included } \\
\text { types of airways }\end{array}$ & $\begin{array}{l}\text { - No airway management } \\
\text { - Prehospital vs. in-hospital }\end{array}$ \\
\hline Outcomes & $\begin{array}{l}\text { Patient Health Outcomes (highest priority) } \\
\text { - Mortality/survival } \\
\text { o To arrival at hospital } \\
\text { o To hospital discharge } \\
\text { o Any period less than or equal to } 30 \text { days post-injury } \\
\text { - Morbidity } \\
\text { o Glasgow Outcome Scale, Glasgow Outcome Scale } \\
\quad \text { Extended, Modified Rankin Scale, Cerebral } \\
\quad \text { Performance Category } \\
\text { o Pneumothorax } \\
\text { o Aspiration pneumonia } \\
\text { - Length of stay } \\
\text { o Hospital length of stay (days) } \\
\text { o ICU length of stay (days) } \\
\text { o ICU-free days } \\
\text { Intermediate outcomes (secondary priority) } \\
\text { - Overall success rate } 1 \\
\text { - First-pass success rate } \\
\text { - Number of prehospital attempts to secure an airway } \\
\text { - EtCO values } \\
\text { - Effective oxygenation } \\
\text { - Effective ventilation } \\
\text { - Definitive Airway Sans Hypoxia/Hypotension on First } \\
\text { Attempt (DASH-1A) } \\
\text { - ROSC } \\
\text { Adverse events/harms } \\
\text { - Vomiting } \\
\text { - Gastric content aspiration } \\
\text { - Hypoxia (SpO }<90 \% \text { ) } \\
\text { - Hyperventilation (EtCO }{ }_{2}<35 \text { ) } \\
\text { - Hypoventilation (EtCO }>45) \\
\text { - Hypotension (low SBP or MAP) } \\
\text { - Oral trauma, airway trauma } \\
\text { - Barotrauma } \\
\text { - Misplaced tube } \\
\text { - Need for additional airway interventions }\end{array}$ & $\begin{array}{l}\text { - Long-term outcomes (more than } \\
30 \text { days post-injury) } \\
\text { - Adrenal inhibition } \\
\text { - Time in field } \\
\text { - Time to resuscitation } \\
\text { - Ventilator associated } \\
\text { - } \mathrm{HCO}_{3} \\
\text { - Tracheal stenosis }\end{array}$ \\
\hline
\end{tabular}




\begin{tabular}{|c|c|c|}
\hline PICOS & Inclusion Criteria & Exclusion Criteria \\
\hline Setting & $\begin{array}{l}\text { - Prehospital } \\
\text { - ED only if needed to fill important gaps where there are } \\
\text { no prehospital studies } \\
\text { - International studies in English language }\end{array}$ & $\begin{array}{l}\text { - Airway studies conducted in } \\
\text { cadaver labs, or simulated } \\
\text { environments; operating rooms, } \\
\text { or inpatient. } \\
\text { - ED studies if prehospital studies } \\
\text { of the topic are available. }\end{array}$ \\
\hline Study Design & $\begin{array}{l}\text { - RCTs } \\
\text { - Prospective comparative studies } \\
\text { - Retrospective comparative studies } \\
\text { - Case control studies }\end{array}$ & $\begin{array}{l}\text { - Systematic reviews (we will use } \\
\text { reference lists to identify studies } \\
\text { for possible inclusion) } \\
\text { - Case series } \\
\text { - Descriptive studies } \\
\text { - Letters to the editor } \\
\text { - Opinion papers } \\
\text { - Studies published prior to } 1990\end{array}$ \\
\hline
\end{tabular}

$\mathrm{BiPAP}=$ bilevel positive airway pressure; $\mathrm{CPAP}=$ continuous positive airway pressure; $\mathrm{DSI}=$ delayed sequence intubation; $\mathrm{ED}$ = emergency department; ICU = intensive care unit; KQ = Key Question; MAP = mean arterial pressure; PICOS = population, interventions, comparaters, outcomes, setting, study design; RCT = randomized controlled trial; RSI = rapid sequence intubation; $\mathrm{SBP}=$ systolic blood pressure

Included studies reported successful airway management with BVM in different ways. Although the BVM approach does not require airway insertion, BVM success was compared to that of SGA and ETI. These comparisons are reported in the Results sections.

Study design. For all Key Questions, we included randomized controlled trials (RCTs). We also included uncontrolled clinical trials, prospective and retrospective comparative observational studies, and case-control studies. For all Key Questions, we excluded case series, descriptive studies, letters to the editor, opinion papers, and case reports. Reference lists from systematic reviews were examined to identify additional studies not captured in our search.

Non-English language studies. We restricted our search to English-language articles, but reviewed English-language abstracts of non-English language articles to identify studies that would otherwise meet inclusion criteria, in order to help assess for the likelihood of language bias.

Process for selecting studies. Pre-established criteria were used to determine eligibility for inclusion and exclusion of abstracts in accordance with the AHRQ Methods Guide for Effectiveness and Comparative Effectiveness Reviews (hereafter "AHRQ Methods Guide"), ${ }^{1}$ based on the Key Questions and PICOS. To ensure accuracy, all excluded abstracts were dual reviewed to confirm exclusion. All abstracts deemed potentially appropriate for inclusion by at least one of the reviewers triggered retrieval of the full-text article. Each full-text article was independently reviewed for eligibility by two team members, including any articles suggested by peer reviewers, or any that arose from the public posting process. During abstract and full-text review, all RCTs and comparative observational studies were retained and categorized according to which Key Questions they address. Authors of a paper who are on the research team did not review their own publications. Disagreements between the two team members were resolved by consensus of the investigators.

Additional selection of studies for Key Question 4. The purpose of Key Question 4 was to look for variation in outcomes from the use of one of the three airway approaches that may not be reported in the head-to-head studies included in Key Questions 1-3. We sought to increase our understanding of how patient characteristics, provider characteristics, and different airway devices and approaches might influence outcomes. After identifying all publications that might meet the criteria for Key Question 4, we eliminated studies for which there was only one paper for the topic, or the studies were too heterogeneous to compare. We further eliminated studies of 
a single intervention reporting outcomes based on variations in Provider Category. The rationale was, there are a sufficient number of studies of the head-to-head comparisons in the first three Key Questions to provide information about how this modifier influences outcome. Of the remaining studies, 32 were meta-analyzed, all about variations in ETI. Twenty-eight studies about ETI and eight studies about SGA were qualitatively analyzed.

\section{Data Extraction}

After studies were selected for inclusion, data were abstracted into categories including study design, year, setting, country, sample size, eligibility criteria, population and clinical characteristics, intervention characteristics, and results relevant to each Key Question as outlined in the PICOS table above. Data were abstracted into an interactive database in order to facilitate meta-analyses. All abstracted data were verified for accuracy and completeness by a second team member. A record of studies excluded at the full-text level with reasons for exclusion was maintained (see Appendix D).

\section{Risk of Bias Assessment of Individual Studies}

Predefined criteria were used to assess the quality of included studies. The criteria used depended on the study design as recommended in the chapter, "Assessing the Risk of Bias of Individual Studies When Comparing Medical Interventions" in the AHRQ Methods Guide. ${ }^{1}$ Randomized controlled trials were evaluated using selected Cochrane risk of bias criteria, ${ }^{2}$ and observational studies were evaluated using criteria developed by the U.S. Preventive Services Task Force. ${ }^{3}$

Risk of Bias ratings were provided based on outcomes used in studies. Therefore, some studies were given an overall rating of "low," "moderate," or "high" risk of bias, whereas other studies were given multiple ratings based on risk of bias specific to different outcomes. Studyspecific ratings can be found in Appendix G.

Studies rated "low" are considered to have the least risk of bias, be of high quality, and their results are generally considered valid. Low risk of bias intervention studies include clear descriptions of the population, setting, interventions, and comparison groups; a valid method for allocating patients to treatment; low dropout rates and clear reporting of dropouts; appropriate means for preventing bias; and appropriate measurement of outcomes.

Studies rated "moderate" are susceptible to some bias, though not enough to necessarily invalidate the results. These studies may not meet all the criteria for a rating of low, but no flaw or combination of flaws is likely to cause major bias. The study may be missing information, making it difficult to assess limitations and potential problems. The moderate category is broad, and studies with this rating vary in their strengths and weaknesses.

Studies rated "high" have significant flaws that imply biases of various types that may invalidate the results. They have a serious or "fatal" flaw (or combination of flaws) in design, analysis, or reporting; large amounts of missing information; discrepancies in reporting; or serious problems in the delivery of the intervention. The results of these studies are at least as likely to reflect flaws in the study design as to show true difference between the compared interventions. We did not exclude studies rated high risk of bias a priori, but high risk of bias studies were considered less reliable than low risk of bias studies when synthesizing the evidence, particularly if there were inconsistencies in study results.

Two team members independently assessed risk of bias. Disagreements were resolved by consensus. 


\section{Data Synthesis and Analysis}

We constructed evidence tables showing study characteristics, results, and quality ratings for all included studies, along with summary tables to highlight the main findings. Results were organized by Key Question, and stratified by major subgroups.

Meta-analyses (Appendix H and Appendix I), using profile-likelihood random effects model ${ }^{4}$ were conducted to summarize data and obtain more precise estimates where there are at least two studies reporting outcomes that were homogeneous enough to provide a meaningful combined estimate. To determine whether meta-analyses were appropriate, we considered the quality of individual studies, the heterogeneity across several variables including patient characteristics, interventions, and outcomes, as well as the completeness of the same reported outcomes. All meta-analyzable outcomes were binary and risk ratio (RR) was the effect measure. Adjusted RRs or odds ratios (OR) were used in the meta-analysis if reported (an adjusted OR was first converted to an adjusted RR). ${ }^{5}$ Otherwise, the RR was calculated from the reported raw numbers. Statistical heterogeneity was assessed using the $\chi^{2}$ test, and the magnitude of heterogeneity using the $I^{2}$ statistic. $^{6}$

The Key Questions were designed to assess the comparative effectiveness and harms by airway intervention, emergency medical services (EMS) personnel, and patient characteristics. Therefore, meta-analyses were stratified by study design (e.g., randomized controlled trials [RCTs] or observational studies), emergency type (e.g. cardiac arrest, trauma), and population age (adult, pediatric, mixed age). Controlled clinical trials (CCTs) were grouped with either RCTs or observational studies in meta-analyses based on the characteristics of the study. If a study provided data for more than one definition of ROSC, we used in order of preference: sustained ROSC, any ROSC, prehospital ROSC. For neurological function, we did not pool across different measures. In primary analyses, we used data from the intent-to-treat (ITT) analysis for RCTs, and if reported, propensity score matched results for observational studies. Studies with mixed age population were grouped with the adult studies for stratification in the primary analyses. Sensitivity analyses were conducted by using other reported data (e.g., data from per-protocol, or as treated analysis for RCTs, unadjusted results), or by excluding studies with outlying results, those rated as high risk of bias, and studies in mixed age populations, as separate analyses.

All analyses were performed by using STATA ${ }^{\circledR} 16.1$ (StataCorp, College Station, TX), and all results were provided with 95 percent confidence intervals (95\% CIs).

\section{Qualitative Synthesis}

Where pooling studies was not appropriate, qualitative syntheses, which include summary tables, tabulations of important study features, and narratives, were created and are presented by Key Questions and outcomes (see Results and Appendix F).

\section{Grading the Strength of the Body of Evidence}

Regardless of whether evidence was synthesized quantitatively or qualitatively, the strength of evidence for each Key Question/body of evidence was initially assessed by one researcher for each clinical outcome (see PICOS, Table A-1) by using the approach described in the AHRQ Methods Guide. ${ }^{1}$ To ensure consistency and validity of the evaluation, the strength of evidence was reviewed by the entire team of investigators prior to assigning a final grade on the following factors: 
- $\quad$ Study risk of bias (low, moderate, or high level of risk of bias)

- Consistency (consistent, inconsistent, or unknown/not applicable)

- $\quad$ Directness (direct or indirect)

- $\quad$ Precision (precise or imprecise)

- $\quad$ Reporting bias (suspected or undetected)

Concern for publication bias was addressed by conducting searches for grey literature, responding to suggestions from public postings, and requesting additional information from authors if needed.

The strength of evidence was assigned an overall grade of high, moderate, low, or insufficient according to a four-level scale by evaluating and weighing the combined results of the above domains (Table A-2).

Table A-2. Definitions of the grades of overall strength of evidence

\begin{tabular}{|l|l|}
\hline Grade & Definition \\
\hline High & $\begin{array}{l}\text { We are very confident that the estimate of effect lies close to the true effect for this outcome. } \\
\text { The body of evidence has few or no deficiencies. We believe that the findings are stable (i.e., } \\
\text { another study would not change the conclusions). }\end{array}$ \\
\hline Moderate & $\begin{array}{l}\text { We are moderately confident that the estimate of effect lies close to the true effect for this } \\
\text { outcome. The body of evidence has some deficiencies. We believe that the findings are likely } \\
\text { to be stable, but some doubt remains. }\end{array}$ \\
\hline Low & $\begin{array}{l}\text { We have limited confidence that the estimate of effect lies close to the true effect for this } \\
\text { outcome. The body of evidence has major or numerous deficiencies (or both). We believe } \\
\text { that additional evidence is needed before concluding either that the findings are stable or that } \\
\text { the estimate of effect is close to the true effect. }\end{array}$ \\
\hline Insufficient & $\begin{array}{l}\text { We have no evidence, we are unable to estimate an effect, or we have no confidence in the } \\
\text { estimate of effect for this outcome. No evidence is available or the body of evidence has } \\
\text { unacceptable deficiencies, precluding reaching a conclusion. }\end{array}$ \\
\hline
\end{tabular}

\section{Appendix A References}

1. Agency for Healthcare Research and Quality. Agency for Healthcare Research and Quality, 2010, Future Research Needs Methods Research Series, https://effectivehealthcare.ahrq.gov/products /future-research-needs-methods/overview. Accessed 12/10/2019.

2. Higgins J, Savović J, Page M, et al. Chapter 8: Assessing risk of bias in a randomized trial. In: Higgins J, Thomas J, Chandler J, Cumpston M, Li T, Page M, et al., eds. Cochrane Handbook for Systematic Reviews of Interventions version 6 (updated July 2019): Cochrane; 2019.

3. United States Preventive Services Task Force. US Preventive Services Task Force. US Preventive Services Task Force Procedure Manual. Rockville, MD: Agency for Healthcare Research and Quality: 2018. https://www.uspreventiveservicestaskforce.o $\mathrm{rg} /$ Page/Name/procedure-manual. Accessed December 26, 2019.
4. Hardy RJ, Thompson SG. A likelihood approach to meta-analysis with random effects. Stat Med. 1996 Mar 30;15(6):61929. doi: $10.1002 /$ (sici)10970258(19960330)15:6<619::Aidsim188>3.0.Co;2-a. PMID: 8731004.

5. Zhang J, Yu KF. What's the relative risk? A method of correcting the odds ratio in cohort studies of common outcomes. JAMA. 1998;280(19):1690-1. doi: 10.1001/jama.280.19.1690.

6. Higgins JP, Thompson SG. Quantifying heterogeneity in a meta-analysis. Stat Med. 2002 Jun 15;21(11):1539-58. doi: 10.1002/sim.1186. PMID: 12111919. 


\section{Appendix B. Literature Flow}

Figure B-1. Literature flow diagram

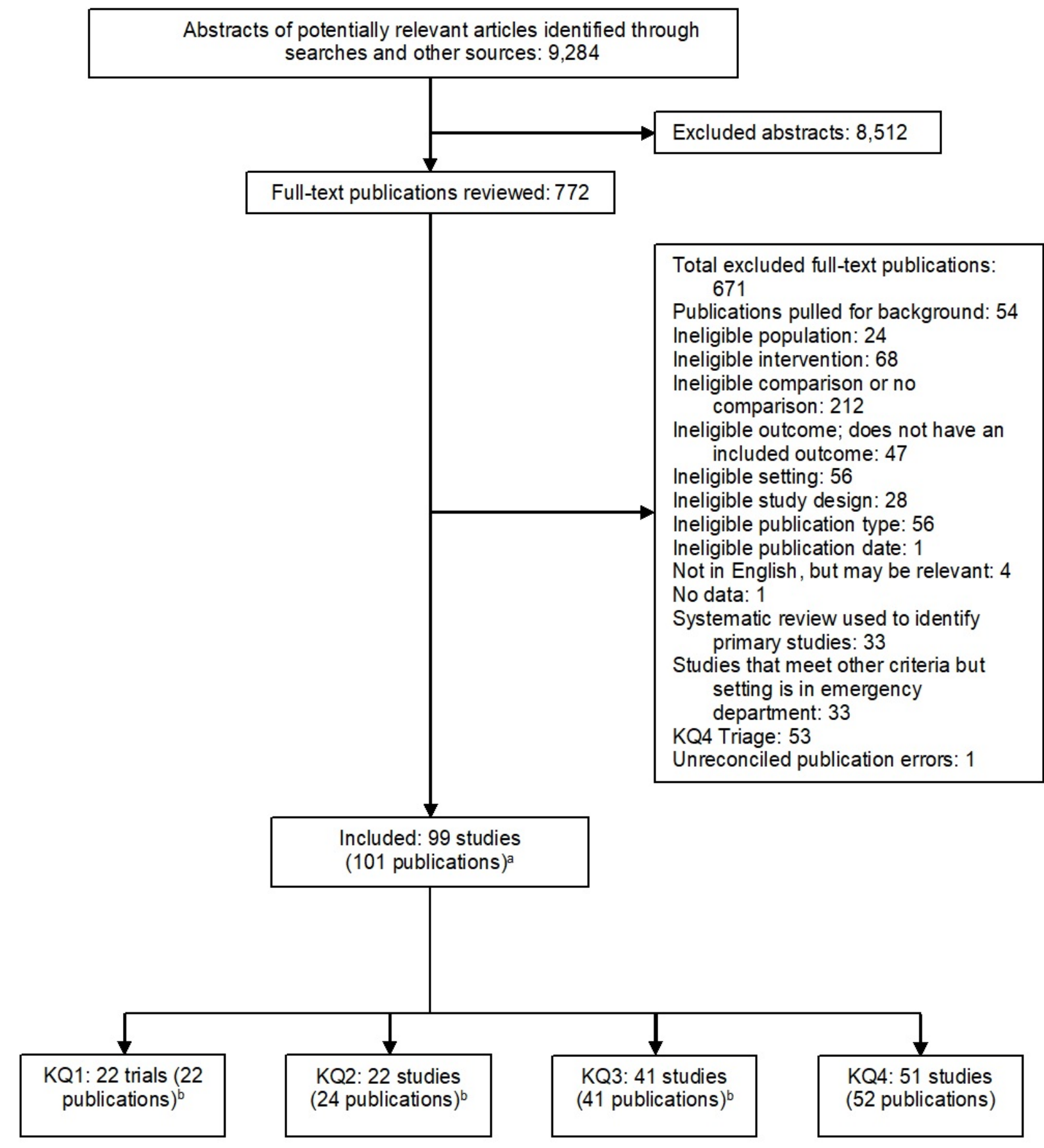

$\mathrm{KQ}=$ Key Question

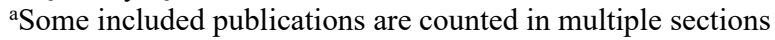

bEvans, 2016 was counted as two trials 


\section{Appendix C. Included Studies}

1. Arima T, Nagata O, Miura T, et al. Comparative analysis of airway scope and Macintosh laryngoscope for intubation primarily for cardiac arrest in prehospital setting. Am J Emerg Med. 2014 Jan;32(1):40-3. doi: 10.1016/j.ajem.2013.09.026. PMID: 24176585.

2. Bartlett RL, Martin SD, McMahon JM, Jr., et al. A field comparison of the pharyngeotracheal lumen airway and the endotracheal tube. J Trauma. 1992 Mar;32(3):280-4. doi: 10.1097/00005373-199203000-00002. PMID: 1548713.

3. Becker TK, Berning AW, Prabhu A, et al. An assessment of ventilation and perfusion markers in out-of-hospital cardiac arrest patients receiving mechanical CPR with endotracheal or supraglottic airways. Resuscitation. 2018 Jan;122:61-4. doi: 10.1016/j.resuscitation.2017.11.054. PMID: 29175355.

4. Behrens NH, Fischer M, Krieger T, et al. Effect of airway management strategies during resuscitation from outof-hospital cardiac arrest on clinical outcome: a registry-based analysis. Resuscitation. 2020 Jul 152:157-64. doi:

10.1016/j.resuscitation.2020.04.015. PMID: 32387124.

5. Bendinelli C, Ku D, Nebauer S, et al. A tale of two cities: prehospital intubation with or without paralysing agents for traumatic brain injury. ANZ J Surg. 2018 May;88(5):455-9. doi: 10.1111/ans.14479. PMID: 29573111.

6. Benger J, Coates D, Davies S, et al. Randomised comparison of the effectiveness of the laryngeal mask airway supreme, i-gel and current practice in the initial airway management of out of hospital cardiac arrest: a feasibility study. Br J Anaesth. 2016 Feb;116(2):262-8. doi:

10.1093/bja/aev477. PMID: 26787796.
7.

Benger JR, Kirby K, Black S, et al. Effect of a strategy of a supraglottic airway device vs tracheal intubation during out-of-hospital cardiac arrest on functional outcome: the AIRWAYS-2 randomized clinical trial. JAMA. 2018 Aug 28;320(8):779-91. doi: 10.1001/jama.2018.11597. PMID: 30167701 .

8. Bernard SA, Nguyen V, Cameron P, et al. Prehospital rapid sequence intubation improves functional outcome for patients with severe traumatic brain injury: a randomized controlled trial. Ann Surg. 2010 Dec;252(6):959-65. doi: 10.1097/SLA.0b013e3181 efc15f. PMID: 21107105

9. Bozeman WP, Kleiner DM, Huggett V. A comparison of rapid-sequence intubation and etomidate-only intubation in the prehospital air medical setting. Prehosp Emerg Care. 2006 JanMar;10(1):8-13. doi: 10.1080/10903120500366854. PMID: 16418085 .

10. Breeman W, Van Vledder MG, Verhofstad MHJ, et al. First attempt success of video versus direct laryngoscopy for endotracheal intubation by ambulance nurses: a prospective observational study. Eur J Trauma Emerg Surg. 2020 Feb 19;19:19. doi: 10.1007/s00068-02001326-z. PMID: 32072225.

11. Bulger EM, Copass MK, Sabath DR, et al. The use of neuromuscular blocking agents to facilitate prehospital intubation does not impair outcome after traumatic brain injury. J Trauma. 2005 Apr;58(4):718-23; discussion 234. doi: 10.1097/01.ta.0000159239.14181.bc. PMID: 15824647.

12. Cady CE, Pirrallo RG. The effect of Combitube use on paramedic experience in endotracheal intubation. Am J Emerg Med. 2005 Nov;23(7):86871. doi: 10.1016/j.ajem.2005.07.013. PMID: 16291443. 
13. Chan JJ, Goh ZX, Koh ZX, et al. Clinical evaluation of the use of laryngeal tube verses laryngeal mask airway for out-of-hospital cardiac arrest by paramedics in Singapore. Singapore Med J. 2020a Aug 17;17:17. doi: 10.11622/smedj.2020119. PMID: 32798357.

14. Chan M, Fehlmann CA, Pasquier M, et al. Endotracheal intubation success rate in an urban, supervised, resident-staffed emergency mobile system: an 11-year retrospective cohort study. J Clin Med. 2020b Jan 16;9(1):16. doi: 10.3390/jcm9010238. PMID: 31963162 .

15. Chiang WC, Hsieh MJ, Chu HL, et al. The effect of successful intubation on patient outcomes after out-of-hospital cardiac arrest in Taipei. Ann Emerg Med. 2018 Mar;71(3):387-96.e2. doi: 10.1016/j.annemergmed.2017.08.008. PMID: 28967516.

16. Chien LC, Hsu HC, Lin CH, et al. Use of an intubating laryngeal mask airway on out-of-hospital cardiac arrest patients in a developing emergency medical service system. J Formos Med Assoc. 2012 Jan;111(1):24-9. doi: 10.1016/j.jfma.2012.01.004. PMID: 22333009.

17. Cooper A, DiScala C, Foltin G, et al. Prehospital endotracheal intubation for severe head injury in children: a reappraisal. Semin Pediatr Surg. 2001 Feb;10(1):3-6. doi: 10.1053/spsu.2001.19379. PMID: 11172563.

18. Cudnik MT, Newgard CD, Daya M, et al. The impact of rapid sequence intubation on trauma patient mortality in attempted prehospital intubation. $\mathbf{J}$ Emerg Med. 2010 Feb;38(2):175-81. doi: 10.1016/j.jemermed.2008.01.022. PMID: 18790586.

19. Davis DP, Peay J, Sise MJ, et al. The impact of prehospital endotracheal intubation on outcome in moderate to severe traumatic brain injury. J Trauma. 2005;58(5):933-9. doi: 10.1097/01.TA.0000162731.53812.58. PMID: 15920406.
20. Delorenzo A, St Clair T, Andrew E, et al. Prehospital rapid sequence intubation by intensive care flight paramedics. Prehosp Emerg Care. 2018 Sep-Oct;22(5):595-601. doi: 10.1080/10903127.2018.1426666. PMID: 29405803.

21. Dos Santos FD, Schnakofsky R, Cascio A, et al. Disposable stainless steel vs plastic laryngoscope blades among paramedics. Am J Emerg Med. 2011 Jul;29(6):590-3. doi: 10.1016/j.ajem.2009.12.022. PMID: 20825833.

22. Ducharme S, Kramer B, Gelbart D, et al. A pilot, prospective, randomized trial of video versus direct laryngoscopy for paramedic endotracheal intubation. Resuscitation. 2017 May;114:121-6. doi:

10.1016/j.resuscitation.2017.03.022. PMID: 28336412.

23. Duckett J, Fell P, Han K, et al. Introduction of the I-gel supraglottic airway device for prehospital airway management in a UK ambulance service. Emerg Med J. 2014 Jun;31(6):505-7. doi: 10.1136/emermed-2012-202126. PMID: 23576232.

24. Eberlein CM, Luther IS, Carpenter TA, et al. First-pass success intubations using video laryngoscopy versus direct laryngoscopy: a retrospective prehospital ambulance service study. Air Med J. 2019 Sep - Oct;38(5):356-8. doi: 10.1016/j.amj.2019.06.004. PMID: 31578974.

25. Eckstein M, Chan L, Schneir A, et al. Effect of prehospital advanced life support on outcomes of major trauma patients. J Trauma. 2000 Apr;48(4):643-8. doi: 10.1097/00005373-200004000-00010. PMID: 10780596.

26. Edwards T, Williams J, Cottee M. Influence of prehospital airway management on neurological outcome in patients transferred to a heart attack centre following out-of-hospital cardiac arrest. Emerg Med Australas. 2019 Feb;31(1):76-82. doi: 10.1111/17426723.13107. PMID: 29752776. 
27. Eich C, Roessler M, Nemeth M, et al. Characteristics and outcome of prehospital paediatric tracheal intubation attended by anaesthesiatrained emergency physicians. Resuscitation. 2009 Dec;80(12):1371-7. doi: 10.1016/j.resuscitation.2009.09.004. PMID: 19804939.

28. Evans CC, Petersen A, Meier EN, et al. Prehospital traumatic cardiac arrest: management and outcomes from the resuscitation outcomes consortium epistry-trauma and PROPHET registries. J Trauma Acute Care Surg. 2016 Aug;81(2):285-93. doi: 10.1097/TA.0000000000001070. PMID: 27070438.

29. Fiala A, Lederer W, Neumayr A, et al. EMT-led laryngeal tube vs. face-mask ventilation during cardiopulmonary resuscitation - a multicenter prospective randomized trial. Scand J Trauma Resusc Emerg Med. 2017 Oct 26;25(1):104. doi: 10.1186/s13049-0170446-1. PMID: 29073915.

30. Fouche PF, Smith K, Jennings PA, et al. The association of paramedic rapid sequence intubation and survival in outof-hospital stroke. Emerg Med J. 2019 Jul;36(7):416-22. doi: 10.1136/emermed-2019-208613. PMID: 31147349 .

31. Frascone RJ, Russi C, Lick C, et al. Comparison of prehospital insertion success rates and time to insertion between standard endotracheal intubation and a supraglottic airway. Resuscitation. 2011 Dec;82(12):152936. doi:

10.1016/j.resuscitation.2011.07.009. PMID: 21763247.

32. Fukuda T, Sekiguchi H, Taira T, et al. Type of advanced airway and survival after pediatric out-of-hospital cardiac arrest. Resuscitation. 2020

May;150:145-53. doi: 10.1016/j.resuscitation.2020.02.005. PMID: 32070779.
33. Gahan K, Studnek JR, Vandeventer S. King LT-D use by urban basic life support first responders as the primary airway device for out-of-hospital cardiac arrest. Resuscitation. 2011 Dec;82(12):1525-8. doi: 10.1016/j.resuscitation.2011.06.036. PMID: 21756859.

34. Gamberini L, Giugni A, Ranieri S, et al. Early-onset ventilator-associated pneumonia in severe traumatic brain injury: is there a relationship with prehospital airway management? J Emerg Med. 2019 Jun;56(6):657-65. doi: 10.1016/j.jemermed.2019.02.005. PMID: 31000428.

35. Garza AG, Algren DA, Gratton MC, et al. Populations at risk for intubation nonattempt and failure in the prehospital setting. Prehosp Emerg Care. 2005 Apr-Jun;9(2):163-6. doi: 10.1080/10903120590924654. PMID: 16036840 .

36. Gausche M, Lewis RJ, Stratton SJ, et al. Effect of out-of-hospital pediatric endotracheal intubation on survival and neurological outcome: a controlled clinical trial. see comment erratum appears in JAMA 2000 Jun 28;283(24): 3204. JAMA. 2000;6:783-90. doi: 10.1001/jama.283.6.783. PMID: 10683058 .

37. Gausche-Hill M, Lewis RJ, Gunter CS, et al. Design and implementation of a controlled trial of pediatric endotracheal intubation in the out-of-hospital setting. Ann Emerg Med. 2000 Oct;36(4):35665. doi: $10.1067 / \mathrm{mem} .2000 .109447$. PMID: 11020685.

38. Gellerfors M, Larsson A, Svensen CH, et al. Use of the Airtraq device for airway management in the prehospital setting--a retrospective study. Scand J Trauma Resusc Emerg Med. 2014 Feb 03;22:10. doi: 10.1186/1757-7241-2210. PMID: 24484856.

39. Hanif MA, Kaji AH, Niemann JT. Advanced airway management does not improve outcome of out-of-hospital cardiac arrest. Acad Emerg Med. 2010 Sep;17(9):926-31. doi: 10.1111/j.15532712.2010.00829.x. PMID: 20836772. 
40. Hankins DG, Carruthers N, Frascone $\mathrm{RJ}$, et al. Complication rates for the esophageal obturator airway and endotracheal tube in the prehospital setting. Prehospital Disaster Med. 1993 Apr-Jun;8(2):117-21. doi: 10.1017/s1049023x00040176. PMID: 10148602 .

41. Hansen M, Wang $\mathrm{H}$, Le N, et al. Prospective evaluation of airway management in pediatric out-of-hospital cardiac arrest. Resuscitation. 2020 Aug 11;11:11. doi: 10.1016/j.resuscitation.2020.08.003. PMID: 32795596.

42. Hansen ML, Lin A, Eriksson C, et al. A comparison of pediatric airway management techniques during out-ofhospital cardiac arrest using the CARES database. Resuscitation. 2017

Nov;120:51-6. doi: 10.1016/j.resuscitation.2017.08.015. PMID: 28838781.

43. Hardy GB, Maddry JK, Ng PC, et al. Impact of prehospital airway management on combat mortality. Am J Emerg Med. 2018 Jun;36(6):1032-5. doi: 10.1016/j.ajem.2018.02.007. PMID: 29691106.

44. Hiltunen $\mathrm{P}$, Jantti $\mathrm{H}$, Silfvast $\mathrm{T}$, et al. Airway management in out-of-hospital cardiac arrest in Finland: current practices and outcomes. Scand J Trauma Resusc Emerg Med. 2016 Apr 12;24:49. doi: 10.1186/s13049-0160235-2. PMID: 27071823.

45. Hoffmann M, Czorlich P, Lehmann W, et al. The impact of prehospital intubation with and without sedation on outcome in trauma patients with a GCS of 8 or less. J Neurosurg Anesthesiol. 2017 Apr;29(2):161-7. doi: 10.1097/ANA.0000000000000275. PMID: 26797107.
46. Hossfeld B, Thierbach S, Allgoewer A, et al. First pass success of tracheal intubation using the C-MAC PM videolaryngoscope as first-line device in prehospital cardiac arrest compared with other emergencies: an observational study. Eur J Anaesthesiol. 2020 Aug 21;21:21. doi: 10.1097/EJA.0000000000001286. PMID: 32833853.

47. Jabre P, Galinski M, Ricard-Hibon A, et al. Out-of-hospital tracheal intubation with single-use versus reusable metal laryngoscope blades: a multicenter randomized controlled trial. Ann Emerg Med. 2011 Mar;57(3):225-31. doi: 10.1016/j.annemergmed.2010.10.011. PMID: 21129822.

48. Jabre P, Leroux B, Brohon S, et al. A comparison of plastic single-use with metallic reusable laryngoscope blades for out-of-hospital tracheal intubation. Ann Emerg Med. 2007 Sep;50(3):25863. doi: 10.1016/j.annemergmed.2007.04.022. PMID: 17583382.

49. Jabre P, Penaloza A, Pinero D, et al. Effect of bag-mask ventilation vs endotracheal intubation during cardiopulmonary resuscitation on neurological outcome after out-ofhospital cardiorespiratory arrest: a randomized clinical trial. JAMA. 2018 Feb 27;319(8):779-87. doi: 10.1001/jama.2018.0156. PMID: 29486039.

50. Jarman AF, Hopkins CL, Hansen JN, et al. Advanced airway type and its association with chest compression interruptions during out-of-hospital cardiac arrest resuscitation attempts. Prehosp Emerg Care. 2017 SepOct;21(5):628-35. doi: 10.1080/10903127.2017.1308611. PMID: 28459305.

51. Jarvis JL, McClure SF, Johns D. EMS intubation improves with king vision video laryngoscopy. Prehosp Emerg Care. 2015;19(4):482-9. doi: 10.3109/10903127.2015.1005259. PMID: 25909850. 
52. Jarvis JL, Wampler D, Wang HE. Association of patient age with first pass success in out-of-hospital advanced airway management. Resuscitation. 2019 Aug;141:136-43. doi:

10.1016/j.resuscitation.2019.06.002. PMID: 31238034.

53. Kajino K, Iwami T, Kitamura T, et al. Comparison of supraglottic airway versus endotracheal intubation for the pre-hospital treatment of out-of-hospital cardiac arrest. Crit Care.

2011;15(5):R236. doi: 10.1186/cc10483. PMID: 21985431.

54. Kang K, Kim T, Ro YS, et al. Prehospital endotracheal intubation and survival after out-of-hospital cardiac arrest: results from the Korean nationwide registry. Am J Emerg Med. $2016 \mathrm{Feb}$;34(2):128-32. doi: 10.1016/j.ajem.2015.09.036. PMID: 26597496.

55. Kreutziger J, Hornung S, Harrer C, et al. Comparing the mcgrath mac video laryngoscope and direct laryngoscopy for prehospital emergency intubation in air rescue patients: a multicenter, randomized, controlled trial. Crit Care Med. 2019 Oct;47(10):1362-70. doi: 10.1097/CCM.0000000000003918. PMID: 31389835.

56. Kwok H, Prekker M, Grabinsky A, et al. Use of rapid sequence intubation predicts improved survival among patients intubated after out-of-hospital cardiac arrest. Resuscitation. 2013 Oct;84(10):1353-8. doi: 10.1016/j.resuscitation.2013.04.015. PMID: 23665389.

57. Louka A, Stevenson C, Jones G, et al. Intubation success after introduction of a quality assurance program using video laryngoscopy. Air Med J. 2018 Sep;37(5):303-5. doi: 10.1016/j.amj.2018.05.001. PMID: 30322632 .
58. Lupton JR, Schmicker RH, Stephens S, et al. Outcomes with the use of bagvalve-mask ventilation during out-ofhospital cardiac arrest in the Pragmatic Airway Resuscitation Trial. Acad Emerg Med. 2020 May;27(5):366-74. doi: 10.1111/acem.13927. PMID: 32220129 .

59. Macke C, Gralla F, Winkelmann M, et al. Increased first pass success with $C$ MAC videolaryngoscopy in prehospital endotracheal intubation-a randomized controlled trial. J Clin Med. 2020 Aug 22;9(9):22. doi: 10.3390/jcm9092719. PMID: 32842705.

60. Maignan M, Koch FX, Kraemer M, et al. Impact of laryngeal tube use on chest compression fraction during out-ofhospital cardiac arrest. A prospective alternate month study. Resuscitation. 2015 Aug;93:113-7. doi: 10.1016/j.resuscitation.2015.06.002. PMID: 26070831.

61. Malinverni S, Bartiaux M, Cavallotto F, et al. Does endotracheal intubation increases chest compression fraction in out of hospital cardiac arrest: a substudy of the CAAM trial. Resuscitation. 2019 Apr;137:35-40. doi: 10.1016/j.resuscitation.2019.01.032. PMID: 30753851.

62. McCall MJ, Reeves M, Skinner M, et al. Paramedic tracheal intubation using the intubating laryngeal mask airway. Prehosp Emerg Care. 2008 JanMar;12(1):30-4. doi: 10.1080/10903120701709803. PMID: 18189174.

63. McMahan S, Ornato JP, Racht EM, et al. Multi-agency, prehospital evaluation of the pharyngeo-tracheal lumen (PTL) airway. Prehospital Disaster Med. 1992 Jan-Mar;7(1):13-8. doi: 10.1017/s1049023x00039145. PMID: 10149691 .

64. McMullan J, Gerecht R, Bonomo J, et al. Airway management and out-ofhospital cardiac arrest outcome in the CARES registry. Resuscitation. 2014 May;85(5):617-22. doi:

10.1016/j.resuscitation.2014.02.007. PMID: 24561079. 
65. Middleton PM, Simpson PM, Thomas RE, et al. Higher insertion success with the i-gel supraglottic airway in out-ofhospital cardiac arrest: a randomised controlled trial. Resuscitation. 2014 Jul;85(7):893-7. doi: 10.1016/j.resuscitation.2014.02.021. PMID: 24594090.

66. Murray JA, Demetriades D, Berne TV, et al. Prehospital intubation in patients with severe head injury. J Trauma. 2000 Dec;49(6):1065-70. doi: 10.1097/00005373-200012000-00015. PMID: 11130490.

67. Myers LA, Gallet CG, Kolb LJ, et al. Determinants of success and failure in prehospital endotracheal intubation.

West J Emerg Med. 2016

Sep;17(5):640-7. doi: 10.5811/westjem.2016.6.29969. PMID: 27625734.

68. Nagao T, Kinoshita K, Sakurai A, et al. Effects of bag-mask versus advanced airway ventilation for patients undergoing prolonged cardiopulmonary resuscitation in pre-hospital setting. J Emerg Med. 2012 Feb;42(2):162-70. doi: 10.1016/j.jemermed.2011.02.020. PMID: 22032811.

69. Noda E, Zaitsu A, Hashizume M, et al. Prognosis of patient with cardiopulmonary arrest transported to Kyushu University Hospital. Fukuoka Igaku Zasshi. 2007 Mar;98(3):73-81. PMID: 17461032.

70. Nwanne T, Jarvis J, Barton D, et al. Advanced airway management success rates in a national cohort of emergency medical services agencies.

Resuscitation. 2020 Jan 01;146:43-9. doi: 10.1016/j.resuscitation.2019.11.006. PMID: 31756361.

71. Ohashi-Fukuda N, Fukuda T, Yahagi N. Effect of pre-hospital advanced airway management for out-of-hospital cardiac arrest caused by respiratory disease: a propensity score-matched study.

Anaesth Intensive Care. 2017

May;45(3):375-83. doi: 10.1177/0310057X1704500314. PMID: 28486897.
72. Olvera DJ, Stuhlmiller DFE, Wolfe A, et al. A continuous quality improvement airway program results in sustained increases in intubation success. Prehosp Emerg Care. 2018 Sep-Oct;22(5):602-7. doi: 10.1080/10903127.2018.1433734. PMID: 29465279.

73. Ono Y, Hayakawa M, Maekawa K, et al. Should laryngeal tubes or masks be used for out-of-hospital cardiac arrest patients? Am J Emerg Med. 2015 Oct;33(10):1360-3. doi: 10.1016/j.ajem.2015.07.043. PMID: 26306437.

74. Powell EK, Hinckley WR, Stolz U, et al. Predictors of definitive airway sans hypoxia/hypotension on first attempt (DASH-1A) success in traumatically injured patients undergoing prehospital intubation. Prehosp Emerg Care. 2019 Oct 07:1-8. doi: 10.1080/10903127.2019.1670299. PMID: 31539287.

75. Prekker ME, Delgado F, Shin J, et al. Pediatric intubation by paramedics in a large emergency medical services system: process, challenges, and outcomes. Ann Emerg Med. 2016 Jan;67(1):20-9.e4. doi: 10.1016/j.annemergmed.2015.07.021. PMID: 26320522.

76. Prekker ME, Kwok H, Shin J, et al. The process of prehospital airway management: challenges and solutions during paramedic endotracheal intubation. Crit Care Med. 2014 Jun;42(6):1372-8. doi: 10.1097/CCM.0000000000000213. PMID: 24589641.

77. Rabitsch W, Schellongowski P, Staudinger T, et al. Comparison of a conventional tracheal airway with the Combitube in an urban emergency medical services system run by physicians. Resuscitation. 2003 Apr;57(1):27-32. doi: 10.1016/s03009572(02)00435-5. PMID: 12668296. 
78. Risse J, Volberg C, Kratz T, et al. Comparison of videolaryngoscopy and direct laryngoscopy by German paramedics during out-of-hospital cardiopulmonary resuscitation; an observational prospective study. BMC Emerg Med. 2020 Mar 23;20(1):22. doi: 10.1186/s12873-020-00316-z. PMID: 32293276 .

79. Rocca B, Crosby E, Maloney J, et al. An assessment of paramedic performance during invasive airway management. Prehosp Emerg Care. 2000 Apr-Jun;4(2):164-7. doi: 10.1080/10903120090941443. PMID: 10782606.

80. Roth D, Hafner C, Aufmesser W, et al. Safety and feasibility of the laryngeal tube when used by EMTs during out-ofhospital cardiac arrest. Am J Emerg Med. 2015 Aug;33(8):1050-5. doi: 10.1016/j.ajem.2015.04.048. PMID: 25957625 .

81. Rumball C, Macdonald D, Barber P, et al. Endotracheal intubation and esophageal tracheal Combitube insertion by regular ambulance attendants: a comparative trial. Prehosp Emerg Care. 2004 Jan-Mar;8(1):15-22. doi: $10.1080 / 312703002764$. PMID: 14691782.

82. Rumball CJ, MacDonald D. The PTL, Combitube, laryngeal mask, and oral airway: a randomized prehospital comparative study of ventilatory device effectiveness and cost-effectiveness in 470 cases of cardiorespiratory arrest. Prehosp Emerg Care. 1997 JanMar;1(1):1-10. doi: 10.1080/10903129708958776. PMID: 9709312.

83. Shin SD, Ahn KO, Song KJ, et al. Outof-hospital airway management and cardiac arrest outcomes: a propensity score matched analysis. Resuscitation. 2012 Mar;83(3):313-9. doi: 10.1016/j.resuscitation.2011.10.028. PMID: 22101202.
84. Sobuwa S, Hartzenberg HB, Geduld H, et al. Outcomes following prehospital airway management in severe traumatic brain injury. S Afr Med J. 2013 Jul 29;103(9):644-6. doi: 10.7196/samj.7035. PMID: 24300684.

85. Sos-Kanto study group. Comparison of arterial blood gases of laryngeal mask airway and bag-valve-mask ventilation in out-of-hospital cardiac arrests. Circ J. 2009 Mar;73(3):490-6. doi: 10.1253/circj.cj-08-0874. PMID: 19194045.

86. Steuerwald MT, Braude DA, Petersen TR, et al. Preliminary report: comparing aspiration rates between prehospital patients managed with extraglottic airway devices and endotracheal intubation. Air Med J. 2018 Jul Aug;37(4):240-3. doi: 10.1016/j.amj.2018.04.004. PMID: 29935702.

87. Stockinger ZT, McSwain NE, Jr. Prehospital endotracheal intubation for trauma does not improve survival over bag-valve-mask ventilation. J Trauma. 2004 Mar;56(3):531-6. doi: 10.1097/01.ta.0000111755.94642.29. PMID: 15128123.

88. Studnek JR, Thestrup L, Vandeventer S, et al. The association between prehospital endotracheal intubation attempts and survival to hospital discharge among out-of-hospital cardiac arrest patients. Acad Emerg Med. 2010 Sep;17(9):918-25. doi: 10.1111/j.15532712.2010.00827.x. PMID: 20836771.

89. Sulzgruber P, Datler P, Sterz F, et al. The impact of airway strategy on the patient outcome after out-of-hospital cardiac arrest: a propensity score matched analysis. Europ Heart J Acute Cardiovasc Care. 2018 Aug;7(5):42331. doi: $10.1177 / 2048872617731894$. PMID: 28948850. 
90. Sunde GA, Heltne JK, Lockey D, et al. Airway management by physicianstaffed helicopter emergency medical services - a prospective, multicentre, observational study of 2,327 patients. Scand J Trauma Resusc Emerg Med. 2015 Aug 07;23:57. doi:

10.1186/s13049-015-0136-9. PMID: 26250700 .

91. Takei Y, Enami M, Yachida T, et al. Tracheal intubation by paramedics under limited indication criteria may improve the short-term outcome of outof-hospital cardiac arrests with noncardiac origin. J Anesth. 2010 Oct;24(5):716-25. doi: 10.1007/s00540010-0974-6. PMID: 20577765.

92. Tanabe S, Ogawa T, Akahane M, et al. Comparison of neurological outcome between tracheal intubation and supraglottic airway device insertion of out-of-hospital cardiac arrest patients: a nationwide, population-based, observational study. J Emerg Med. 2013 Feb;44(2):389-97. doi: 10.1016/j.jemermed.2012.02.026. PMID: 22541878.

93. Trimmel H, Kreutziger J, Fertsak G, et al. Use of the Airtraq laryngoscope for emergency intubation in the prehospital setting: a randomized control trial. Crit Care Med. 2011 Mar;39(3):489-93. doi: 10.1097/CCM.0b013e318206b69b. PMID: 21169822.

94. Trimmel H, Kreutziger J, Fitzka R, et al. Use of the glidescope ranger video laryngoscope for emergency intubation in the prehospital setting: a randomized control trial. Crit Care Med. 2016 Jul;44(7):e470-6. doi: 10.1097/CCM.0000000000001669. PMID: 27002277.

95. Vilke GM, Hoyt DB, Epperson M, et al. Intubation techniques in the helicopter. J Emerg Med. 1994 MarApr;12(2):217-24. doi: 10.1016/07364679(94)90702-1. PMID: 8207159.
96. Wang HE, Schmicker RH, Daya MR, et al. Effect of a strategy of initial laryngeal tube insertion vs endotracheal intubation on 72-hour survival in adults with out-of-hospital cardiac arrest: a randomized clinical trial. JAMA. 2018 Aug 28;320(8):769-78. doi: 10.1001/jama.2018.7044. PMID: 30167699.

97. Wang HE, Szydlo D, Stouffer JA, et al. Endotracheal intubation versus supraglottic airway insertion in out-ofhospital cardiac arrest. Resuscitation. 2012 Sep;83(9):1061-6. doi: 10.1016/j.resuscitation.2012.05.018. PMID: 22664746.

98. Wang HE, Yealy DM. How many attempts are required to accomplish outof-hospital endotracheal intubation? Acad Emerg Med. 2006 Apr;13(4):3727. doi: 10.1197/j.aem.2005.11.001. PMID: 16531595.

99. Wayne MA, McDonnell M. Comparison of traditional versus video laryngoscopy in out-of-hospital tracheal intubation. Prehosp Emerg Care. 2010 Apr-Jun;14(2):278-82. doi: 10.3109/10903120903537189. PMID: 20199237.

100. Yanagawa Y, Sakamoto T. Analysis of prehospital care for cardiac arrest in an urban setting in Japan. J Emerg Med. 2010 Apr;38(3):340-5. doi: 10.1016/j.jemermed.2008.04.037. PMID: 18993021.

101. Yuksen C, Phattharapornjaroen P, Kreethep W, et al. Bag-valve mask versus endotracheal intubation in outof-hospital cardiac arrest on return of spontaneous circulation: a national database study. Open Access Emerg Med. 2020;12:43-6. doi: 10.2147/OAEM.S229356. PMID: 32210644. 


\section{Appendix D. Excluded Studies}

1. Bag-mask ventilation failed to improve on endotracheal intubation in cardiac arrest. RT: The Journal for Respiratory Care Practitioners. 2017;30(6):32-. Exclusion: Ineligible publication type.

2. Laryngeal vs endotracheal tubes after out of hospital cardiac arrest. RT: The Journal for Respiratory Care Practitioners.

2018;31(8):6-8. Exclusion: Ineligible study design.

3. Abdelgadir IS, Phillips RS, Singh D, et al. Videolaryngoscopy versus direct laryngoscopy for tracheal intubation in children (excluding neonates). Cochrane Database Syst Rev. 2017 May 24;5:CD011413. doi: 10.1002/14651858.CD011413.pub2. PMID: 28539007. Exclusion: Systematic review used to identify primary studies.

4. Abid ES, McNamara J, Hall P, et al. The impact of videolaryngoscopy on endotracheal intubation success by a pediatric/neonatal critical care transport team. Prehosp Emerg Care. 2020 May 15:18. doi: 10.1080/10903127.2020.1761492. PMID: 32347776. Exclusion: Ineligible setting.

5. Abrons RO, Zimmerman MB, El-Hattab YMS. Nasotracheal intubation over a bougie vs. non-bougie intubation: a prospective randomised, controlled trial in older children and adults using videolaryngoscopy. Anaesthesia. 2017 Dec;72(12):1491-500. doi: 10.1111/anae.14029. PMID: 28921537. Exclusion: Ineligible intervention.

6. Adams BD, Cuniowski PA, Muck A, et al. Registry of emergency airways arriving at combat hospitals. J Trauma. 2008 Jun;64(6):1548-54. doi: 10.1097/TA.0b013e3181728c41. PMID: 18545122. Exclusion: Ineligible comparison or no comparison.

7. Adams JN, Sirel J, Marsden K, et al. Heartstart Scotland: the use of paramedic skills in out of hospital resuscitation. Heart. 1997 Oct;78(4):399-402. doi: 10.1136/hrt.78.4.399. PMID: 9404259. Exclusion: Ineligible comparison or no comparison.
8. Adams K, Scott R, Perkin RM, et al. Comparison of intubation skills between interfacility transport team members. Pediatr Emerg Care. 2000 Feb;16(1):5-8. doi: 10.1097/00006565-200002000-00002. PMID: 10698134. Exclusion: Ineligible setting.

9. Adhikari DD, Mahathi K, Ghosh U, et al. Impact of pre-hospital care on the outcome of children arriving with agonal breathing to a pediatric emergency service in South India. J Family Med Prim Care. 2016 JulSep;5(3):625-30. doi: 10.4103/22494863.197321. PMID: 28217595. Exclusion: Ineligible comparison or no comparison.

10. Adib-Hajbaghery M, Maghaminejad F. Epidemiology of patients with multiple trauma and the quality of their prehospital respiration management in kashan, iran: six months assessment. Arch Trauma Res. 2014 Jun;3(2):e17150. doi: 10.5812/atr.17150. PMID: 25147774. Exclusion: Ineligible outcome.

11. Adnet F, Cydulka RK, Lapandry C. Emergency tracheal intubation of patients lying supine on the ground: influence of operator body position. Can J Anaesth. 1998 Mar;45(3):266-9. doi: 10.1007/BF03012914. PMID: 9579267. Exclusion: KQ4 Triage.

12. Adnet F, Jouriles NJ, Le Toumelin P, et al. Survey of out-of-hospital emergency intubations in the French prehospital medical system: a multicenter study. Ann Emerg Med. 1998b Oct;32(4):454-60. doi: 10.1016/s0196-0644(98)70175-1. PMID: 9774930. Exclusion: Ineligible comparison or no comparison.

13. Agro F, Frass M, Benumof J, et al. The esophageal tracheal combitube as a noninvasive alternative to endotracheal intubation. A review. Minerva Anestesiol. 2001 Dec;67(12):863-74. PMID: 11815747. Exclusion: Ineligible publication type.

14. Agro FE, Cataldo R, Mattei A. New devices and techniques for airway management. Minerva Anestesiol. 2009 Mar;75(3):141-9. PMID: 18946431. Exclusion: Ineligible publication type. 
15. Albrecht E, Yersin B, Spahn D, et al. Success rate of airway management by residents in a pre-hospital emergency setting: a retrospective study. European Journal of Trauma. 2006;32(6):516-22. Exclusion: Ineligible comparison or no comparison.

16. Al-Ghamdi AA, El Tahan MR, Khidr AM. Comparison of the Macintosh, GlideScope, Airtraq, and King VisionTM laryngoscopes in routine airway management. Minerva Anestesiol. 2016 Dec;82(12):1278-87. PMID: 27103030. Exclusion: Ineligible setting.

17. Ali QE, Das B, Amir SH, et al. Comparison of the Airtraq and McCoy laryngoscopes using a rigid neck collar in patients with simulated difficult laryngoscopy. J Clin Anesth. 2014 May;26(3):199-203. doi: 10.1016/j.jclinane.2013.10.012. PMID: 24809787. Exclusion: Ineligible comparison or no comparison.

18. Alikhanizadeh H, Sabouhi F, Haghani F, et al. A study of emergency medical technicians' cognitive and practical skills in airway management and its relationship with some related factors in emergency medical centers selected in Isfahan in the year 20152016. Annals of Tropical Medicine \& Public Health. 2017;10(6):1691-7. Exclusion: Ineligible comparison or no comparison.

19. Allen CJ, Teisch LF, Meizoso JP, et al. Prehospital care and transportation of pediatric trauma patients. J Surg Res. 2015 Aug;197(2):240-6. doi: 10.1016/j.jss.2015.03.005. PMID: 25846726. Exclusion: Ineligible comparison or no comparison.

20. Allen TL, Delbridge TR, Stevens MH, et al. Intubation success rates by air ambulance personnel during 12-versus 24-hour shifts: does fatigue make a difference? Prehosp Emerg Care. 2001 Oct-Dec;5(4):340-3. doi: 10.1080/10903120190939481. PMID: 11642582. Exclusion: Ineligible outcome.

21. Alter SM, Haim ED, Sullivan AH, et al. Intubation of prehospital patients with curved laryngoscope blade is more successful than with straight blade. Am J Emerg Med. 2018 Oct;36(10):1807-9. doi: 10.1016/j.ajem.2018.01.100. PMID: 29463438. Exclusion: KQ4 Triage.

22. Altun D, Ali A, Camci E, et al. Haemodynamic response to four different laryngoscopes. Turk J Anaesthesiol Reanim. 2018 Dec;46(6):434-40. doi: 10.5152/TJAR.2018.59265. PMID: 30505605. Exclusion: Ineligible setting.
23. Andersen LW, Raymond TT, Berg RA, et al. Association between tracheal intubation during pediatric in-hospital cardiac arrest and survival. JAMA. 2016 Nov

1;316(17):1786-97. doi: 10.1001/jama.2016.14486. PMID: 27701623. Exclusion: Ineligible setting.

24. Andrusiek DL, Szydlo D, May S, et al. A comparison of invasive airway management and rates of pneumonia in prehospital and hospital settings. Prehosp Emerg Care. 2015;19(4):475-81. doi: 10.3109/10903127.2015.1005263. PMID: 25909984. Exclusion: Ineligible comparison or no comparison.

25. Angerman S, Kirves H, Nurmi J. A beforeand-after observational study of a protocol for use of the C-MAC videolaryngoscope with a Frova introducer in pre-hospital rapid sequence intubation. Anaesthesia. 2018 Mar;73(3):348-55. doi: 10.1111/anae.14182. PMID: 29315473. Exclusion: Ineligible comparison or no comparison.

26. Anonymous. Study challenges standard airway management during cardiac arrests. Bmj. 2013 Jan 16;346:f253. doi: 10.1136/bmj.f253. PMID: 23325873. Exclusion: Ineligible publication type.

27. Archambault P, Dionne CE, Lortie G, et al. Adrenal inhibition following a single dose of etomidate in intubated traumatic brain injury victims. CJEM, Can. 2012 Sep;14(5):27082. doi: 10.2310/8000.2012.110560. PMID: 22967694. Exclusion: Ineligible outcome.

28. Arreola-Risa C, Mock C, Herrera-Escamilla AJ, et al. Cost-effectiveness and benefit of alternatives to improve training for prehospital trauma care in Mexico. Prehospital Disaster Med. 2004 OctDec;19(4):318-25. doi: 10.1017/s1049023x00001953. PMID: 15645628. Exclusion: Ineligible outcome.

29. Asai T, Uchiyama Y, Yamamoto K, et al. Evaluation of the disposable Vital View laryngoscope apparatus. Anaesthesia. 2001 Apr;56(4):342-5. doi: 10.1046/j.13652044.2001.01906.x. PMID: 11284820. Exclusion: Ineligible setting.

30. Atherton GL, Johnson JC. Ability of paramedics to use the Combitube in prehospital cardiac arrest. Ann Emerg Med. 1993 Aug;22(8):1263-8. doi: 10.1016/s0196-0644(05)80104-0. PMID: 8333625. Exclusion: Ineligible comparison or no comparison. 
31. Atlas GM. A comparison of fiberopticcompatible oral airways. J Clin Anesth. 2004 Feb;16(1):66-73. doi: 10.1016/j.jclinane.2003.04.003. PMID: 14984864. Exclusion: Ineligible publication type.

32. Ausserer J, Moritz E, Stroehle M, et al. Physician staffed helicopter emergency medical systems can provide advanced trauma life support in mountainous and remote areas. Injury. $2017 \mathrm{Jan}$;48(1):20-5. doi: 10.1016/j.injury.2016.09.005. PMID: 27650943. Exclusion: Ineligible intervention.

33. Aydogmus MT, Eksioglu B, Oba S, et al. Comparison of laryngeal mask airway supreme and laryngeal mask airway proseal for laryngopharyngeal trauma and postoperative morbidity in children. Braz $\mathrm{J}$ Anesthesiol. 2013 Nov-Dec;63(6):445-9. doi: 10.1016/j.bjane.2012.08.004. PMID: 24565340. Exclusion: Ineligible setting.

34. Babl FE, Vinci RJ, Bauchner H, et al. Pediatric pre-hospital advanced life support care in an urban setting. Pediatr Emerg Care. 2001 Feb;17(1):5-9. doi: 10.1097/00006565200102000-00002. PMID: 11265910. Exclusion: Ineligible comparison or no comparison.

35. Baekgaard JS, Eskesen TG, Sillesen M, et al. Ketamine as a rapid sequence induction agent in the trauma population: a systematic review. Anesth Analg. 2019

Mar;128(3):504-10. doi: 10.1213/ANE.0000000000003568. PMID: 29944524. Exclusion: Systematic review used to identify primary studies.

36. Baekgaard JS, Triba MN, Brandeis M, et al. Early-onset pneumonia following bag-mask ventilation versus endotracheal intubation during cardiopulmonary resuscitation: a substudy of the CAAM trial. Resuscitation. 2020 Sep;154:12-8. doi:

10.1016/j.resuscitation.2020.06.011. PMID: 32629091. Exclusion: Ineligible outcome.

37. Baker TW, King W, Soto W, et al. The efficacy of pediatric advanced life support training in emergency medical service providers. Pediatr Emerg Care. 2009 Aug;25(8):508-12. doi: 10.1097/PEC.0b013e3181b0a0da. PMID: 19633586. Exclusion: KQ4 Triage.

38. Bankole S, Asuncion A, Ross S, et al. First responder performance in pediatric trauma: a comparison with an adult cohort. Pediatr Crit Care Med. 2011 Jul;12(4):e166-70. doi: 10.1097/PCC.0b013e3181f36f6e. PMID: 20729789. Exclusion: KQ4 Triage.
39. Bard MR, Goettler CE, Schenarts PJ, et al. Language barrier leads to the unnecessary intubation of trauma patients. Am Surg. 2004 Sep;70(9):783-6. PMID: 15481294. Exclusion: Ineligible outcome.

40. Barnard EB, Moy RJ, Kehoe AD, et al. Rapid sequence induction of anaesthesia via the intraosseous route: a prospective observational study. Emerg Med J. 2015 Jun;32(6):449-52. doi: 10.1136/emermed2014-203740. PMID: 24963149. Exclusion: Ineligible intervention.

41. Barrett B, Guly HR. How long does it take to perform procedures on scene? Prehospital Immediate Care. 2000;4(1):25-9. Exclusion: Ineligible publication type.

42. Belpomme V, Ricard-Hibon A, Devoir C, et al. Correlation of arterial PCO2 and PETCO2 in prehospital controlled ventilation. Am J Emerg Med. 2005 Nov;23(7):852-9. doi: 10.1016/j.ajem.2005.04.011. PMID: 16291440. Exclusion: Ineligible comparison or no comparison.

43. Ben-Abraham R, Shapira I, Kalmovich B, et al. Supraglottic devices: a possible role in prehospital toxic mass casualty event? J Med. 2004;35(1-6):105-14. PMID: 18084869. Exclusion: Ineligible publication type.

44. Benger JR, Voss S, Coates D, et al. Randomised comparison of the effectiveness of the laryngeal mask airway supreme, i-gel and current practice in the initial airway management of prehospital cardiac arrest (REVIVE-Airways): a feasibility study research protocol. BMJ Open. 2013;3(2)doi: 10.1136/bmjopen-2012-002467. PMID: 23408081. Exclusion: Ineligible outcome.

45. Benoit JL, Gerecht RB, Steuerwald MT, et al. Endotracheal intubation versus supraglottic airway placement in out-ofhospital cardiac arrest: a meta-analysis. Resuscitation. 2015a Aug;93:20-6. doi: 10.1016/j.resuscitation.2015.05.007. PMID: 26006743. Exclusion: Systematic review used to identify primary studies.

46. Benoit JL, McMullan JT, Wang HE, et al. Timing of advanced airway placement after witnessed out-of-hospital cardiac arrest. Prehosp Emerg Care. 2019 NovDec;23(6):838-46. doi: 10.1080/10903127.2019.1595236. PMID: 30912467. Exclusion: Ineligible outcome. 
47. Berlac P, Hyldmo PK, Kongstad P, et al. Pre-hospital airway management: guidelines from a task force from the Scandinavian Society for Anaesthesiology and Intensive Care Medicine. Acta Anaesthesiol Scand. 2008 Aug;52(7):897-907. doi:

10.1111/j.1399-6576.2008.01673.x. PMID: 18702752. Exclusion: Ineligible publication type.

48. Bernard S, Smith K, Foster S, et al. The use of rapid sequence intubation by ambulance paramedics for patients with severe head injury. Emerg Med (Fremantle). 2002 Dec;14(4):406-11. doi: 10.1046/j.14422026.2002.00382.x. PMID: 12534484. Exclusion: Ineligible comparison or no comparison.

49. Bernhard M, Mohr S, Weigand MA, et al. Developing the skill of endotracheal intubation: implication for emergency medicine. Acta Anaesthesiol Scand. 2012 Feb;56(2):164-71. doi: 10.1111/j.13996576.2011.02547.x. PMID: 22060976. Exclusion: Ineligible setting.

50. Berns SD, Patel RI, Chamberlain JM. Oral intubation using a lighted stylet vs direct laryngoscopy in older children with cervical immobilization. Acad Emerg Med. 1996 Jan;3(1):34-40. doi: 10.1111/j.15532712.1996.tb03300.x. PMID: 8749965. Exclusion: Ineligible setting.

51. Bertrand C, Hemery F, Carli P, et al. Constant flow insufflation of oxygen as the sole mode of ventilation during out-ofhospital cardiac arrest. Intensive Care Med. 2006 Jun;32(6):843-51. doi: 10.1007/s00134-006-0137-2. PMID: 16715326. Exclusion: Ineligible comparison or no comparison.

52. Bharti N, Mohanty B, Bithal PK, et al. Intraocular pressure changes associated with intubation with the intubating laryngeal mask airway compared with conventional laryngoscopy. Anaesth Intensive Care. 2008 May;36(3):431-5. doi: 10.1177/0310057x0803600315. PMID: 18564806. Exclusion: Ineligible outcome.

53. Bhende MS, LaCovey DC. End-tidal carbon dioxide monitoring in the prehospital setting. Prehosp Emerg Care. 2001 AprJun;5(2):208-13. doi: 10.1080/10903120190940146. PMID: 11339734. Exclusion: Ineligible publication type.
54. Biarent D, Bingham R, Eich C, et al. European Resuscitation Council Guidelines for Resuscitation 2010 section 6. Paediatric life support. Resuscitation. 2010

Oct;81(10):1364-88. doi: 10.1016/j.resuscitation.2010.08.012. PMID: 20956047. Exclusion: Ineligible publication type.

55. Bieler D, Franke A, Lefering R, et al. Does the presence of an emergency physician influence pre-hospital time, pre-hospital interventions and the mortality of severely injured patients? A matched-pair analysis based on the trauma registry of the German Trauma Society (TraumaRegister DGU). Injury. 2017 Jan;48(1):32-40. doi: 10.1016/j.injury.2016.08.015. PMID: 27586065. Exclusion: KQ4 Triage.

56. Bilge S, Aydin A, Bilge M, et al. A study on the tactical safety of endotracheal intubation under darkness. Mil Med. 2017

Jul;182(7):e1722-e5. doi: 10.7205/MILMED-D-16-00407. PMID: 28810964. Exclusion: Ineligible outcome.

57. Billups K, Larrimore A, Li J, et al. Impact of paralytic agent on postintubation sedation. Air Med J. 2019 Jan - Feb;38(1):39-44. doi: 10.1016/j.amj.2018.09.006. PMID: 30711084. Exclusion: Ineligible population.

58. Binks MJ, Holyoak RS, Melhuish TM, et al. Apneic oxygenation during intubation in the emergency department and during retrieval: a systematic review and meta-analysis. Am J Emerg Med. 2017 Oct;35(10):1542-6. doi: 10.1016/j.ajem.2017.06.046. PMID: 28684195. Exclusion: Ineligible intervention.

59. Bjoernsen LP, Lindsay B. Video laryngoscopy in the prehospital setting. Prehospital Disaster Med. 2009 MayJun;24(3):265-70. doi: 10.1017/s1049023x00006919. PMID: 19618365. Exclusion: Ineligible publication type.

60. Blackburn MB, April MD, Brown DJ, et al. Prehospital airway procedures performed in trauma patients by ground forces in Afghanistan. J Trauma Acute Care Surg. 2018 Jul;85(1S Suppl 2):S154-S60. doi: 10.1097/TA.0000000000001866. PMID: 29521802. Exclusion: Ineligible comparison or no comparison. 
61. Bloomer R, Burns BJ, Ware S. Improving documentation in prehospital rapid sequence intubation: investigating the use of a dedicated airway registry form. Emerg Med J. 2013 Apr;30(4):324-6. doi: 10.1136/emermed-2011-200715. PMID: 22505304. Exclusion: Ineligible intervention.

62. Bobrow BJ, Clark LL, Ewy GA, et al. Minimally interrupted cardiac resuscitation by emergency medical services for out-ofhospital cardiac arrest. JAMA. 2008 Mar 12;299(10):1158-65. doi: 10.1001/jama.299.10.1158. PMID: 18334691. Exclusion: Ineligible intervention.

63. Bobrow BJ, Ewy GA, Clark L, et al. Passive oxygen insufflation is superior to bag-valvemask ventilation for witnessed ventricular fibrillation out-of-hospital cardiac arrest. Ann Emerg Med. 2009 Nov;54(5):65662.e1. doi:

10.1016/j.annemergmed.2009.06.011. PMID: 19660833. Exclusion: Ineligible comparison or no comparison.

64. Bochicchio GV, Ilahi O, Joshi M, et al. Endotracheal intubation in the field does not improve outcome in trauma patients who present without an acutely lethal traumatic brain injury. J Trauma. 2003 Feb;54(2):30711. doi: 10.1097/01.TA.0000046252.97590.BE. PMID: 12579056. Exclusion: Ineligible comparison or no comparison.

65. Boehringer B, Choate M, Hurwitz S, et al. Impact of video laryngoscopy on advanced airway management by critical care transport paramedics and nurses using the CMAC pocket monitor. Biomed Res Int. 2015;2015:821302. doi: 10.1155/2015/821302. PMID: 26167501. Exclusion: Ineligible setting.

66. Bosch J, de Nooij J, de Visser M, et al. Prehospital use in emergency patients of a laryngeal mask airway by ambulance paramedics is a safe and effective alternative for endotracheal intubation. Emerg Med J. 2014 Sep;31(9):750-3. doi:

10.1136/emermed-2012-202283. PMID:

23771898. Exclusion: Ineligible comparison or no comparison.
67. Bossers SM, Schwarte LA, Loer SA, et al. Experience in prehospital endotracheal intubation significantly influences mortality of patients with severe traumatic brain injury: a systematic review and metaanalysis. PLoS ONE. 2015;10(10):e0141034. doi: 10.1371/journal.pone.0141034. PMID: 26496440. Exclusion: Systematic review used to identify primary studies.

68. Boswell WC, McElveen N, Sharp M, et al. Analysis of prehospital pediatric and adult intubation. Air Med J. 1995 JulSep;14(3):125-7; discussion 7-8. doi: 10.1016/1067-991x(95)90513-8. PMID: 10151151. Exclusion: Ineligible comparison or no comparison.

69. Boyle MF, Hatton D, Sheets C. Surgical cricothyrotomy performed by air ambulance flight nurses: a 5-year experience. J Emerg Med. 1993 Jan-Feb;11(1):41-5. doi: 10.1016/0736-4679(93)90008-u. PMID: 8445185. Exclusion: Ineligible intervention.

70. Brainard C. Whose tube is it? San Diego ensures complete airway control via airway paramedics \& capnography. JEMS. 2006;31(3):62-8. Exclusion: Ineligible publication type.

71. Brandling J, Rhys M, Thomas M, et al. An exploration of the views of paramedics regarding airway management. Scand J Trauma Resusc Emerg Med. 2016 Apr 27;24:56. doi: 10.1186/s13049-016-0243-2. PMID: 27121111. Exclusion: Ineligible study design.

72. Braude D, Dixon D, Torres M, et al. Brief research report: prehospital rapid sequence airway. Prehosp Emerg Care. 2020 Jul 22:1 5. doi: 10.1080/10903127.2020.1792015. PMID: 32628568. Exclusion: Ineligible study design, Ineligible comparison or no comparison.

73. Brazinova A, Majdan M, Leitgeb J, et al. Factors that may improve outcomes of early traumatic brain injury care: prospective multicenter study in Austria. Scand J Trauma Resusc Emerg Med. $2015 \mathrm{Jul}$ 16;23:53. doi: 10.1186/s13049-015-0133-z. PMID: 26179747. Exclusion: Ineligible intervention.

74. Breckwoldt J, Klemstein S, Brunne B, et al. Difficult prehospital endotracheal intubation - predisposing factors in a physician based EMS. Resuscitation. 2011 Dec;82(12):151924. doi: 10.1016/j.resuscitation.2011.06.028. PMID: 21749908. Exclusion: Ineligible outcome. 
75. Brimacombe J, Keller C. Gum elastic bougie-guided insertion of the ProSeal laryngeal mask airway. Anaesth Intensive Care. 2004a Oct;32(5):681-4. doi: 10.1177/0310057X0403200514. PMID: 15535495. Exclusion: Ineligible setting.

76. Brimacombe J, von Goedecke A, Keller C, et al. The laryngeal mask airway Unique versus the Soft Seal laryngeal mask: a randomized, crossover study in paralyzed, anesthetized patients. Anesth Analg. 2004b Nov;99(5):1560-3; table of contents. doi: 10.1213/01.ANE.0000133916.10017.6D. PMID: 15502065. Exclusion: Ineligible setting.

77. Broderick ED, Reed JJ. EMS, Pros and Cons of Drug-assisted Intubation. StatPearls Publishing. 2018 Jan;01:01. PMID: 30085511. Exclusion: Ineligible publication type.

78. Brown CA, 3rd, Cox K, Hurwitz S, et al. 4,871 emergency airway encounters by air medical providers: a report of the air transport emergency airway management (NEAR VI: "A-TEAM") project. West J Emerg Med. 2014 Mar;15(2):188-93. doi: 10.5811/westjem.2013.11.18549. PMID: 24672610. Exclusion: Ineligible setting.

79. Brown JB, Rosengart MR, Forsythe RM, et al. Not all prehospital time is equal: Influence of scene time on mortality. J Trauma Acute Care Surg. 2016 Jul;81(1):93100. doi: 10.1097/TA.0000000000000999. PMID: 26886000. Exclusion: Ineligible outcome.

80. Brown LH, Fullagar CJ. Research review. Comparison of etomidate \& midazolam for RSI. JEMS. 2004;29(10):42-4. Exclusion: Ineligible publication type.

81. Brown LH, Hubble MW, Wilfong DA, et al. Airway management in the air medical setting. Air Med J. 2011 MayJun;30(3):140-8. doi: 10.1016/j.amj.2010.11.010. PMID: 21549286. Exclusion: Systematic review used to identify primary studies.

82. Brown LH, Prasad NH. Research review. Prehospital intubation outcomes for TBI. JEMS: Journal of Emergency Medical Services. 2005;30(2):40-1. Exclusion: Ineligible intervention.
83. Bruder EA, Ball IM, Ridi S, et al. Single induction dose of etomidate versus other induction agents for endotracheal intubation in critically ill patients. Cochrane Database Syst Rev. 2015 Jan 08;1:CD010225. doi: 10.1002/14651858.CD010225.pub2. PMID: 25568981. Exclusion: Systematic review used to identify primary studies.

84. Buis ML, Maissan IM, Hoeks SE, et al. Defining the learning curve for endotracheal intubation using direct laryngoscopy: a systematic review. Resuscitation. 2016 Feb;99:63-71. doi: 10.1016/j.resuscitation.2015.11.005. PMID: 26711127. Exclusion: Systematic review used to identify primary studies.

85. Bukur M, Kurtovic S, Berry C, et al. Prehospital intubation is associated with increased mortality after traumatic brain injury. J Surg Res. 2011 Sep;170(1):e11721. doi: 10.1016/j.jss.2011.04.005. PMID: 21601884. Exclusion: Ineligible comparison or no comparison.

86. Bulger EM, Copass MK, Maier RV, et al. An analysis of advanced prehospital airway management. J Emerg Med. 2002 Aug;23(2):183-9. doi: 10.1016/s07364679(02)00490-0. PMID: 12359289. Exclusion: Ineligible comparison or no comparison.

87. Burnett AM, Frascone RJ, Wewerka SS, et al. Comparison of success rates between two video laryngoscope systems used in a prehospital clinical trial. Prehosp Emerg Care. 2014 Apr-Jun;18(2):231-8. doi: 10.3109/10903127.2013.851309. PMID: 24400965. Exclusion: KQ4 Triage.

88. Burnett AM, Peterson BK, Stellpflug SJ, et al. The association between ketamine given for prehospital chemical restraint with intubation and hospital admission. Am J Emerg Med. 2015 Jan;33(1):76-9. doi: 10.1016/j.ajem.2014.10.016. PMID: 25455046. Exclusion: Ineligible comparison or no comparison.

89. Burns B, Habig K, Eason H, et al. Difficult intubation factors in prehospital rapid sequence intubation by an australian helicopter emergency medical service. Air Med J. 2016 Jan-Feb;35(1):28-32. doi: 10.1016/j.amj.2015.10.002. PMID: 26856657. Exclusion: Ineligible comparison or no comparison. 
90. Burns BJ, Watterson JB, Ware S, et al. Analysis of out-of-hospital pediatric intubation by an Australian helicopter emergency medical service. Ann Emerg Med. 2017 Dec;70(6):773-82.e4. doi: 10.1016/j.annemergmed.2017.03.020. PMID: 28460858. Exclusion: Ineligible population.

91. Burton JH, Baumann MR, Maoz T, et al. Endotracheal intubation in a rural EMS state: procedure utilization and impact of skills maintenance guidelines. Prehosp Emerg Care. 2003 Jul-Sep;7(3):352-6. doi: 10.1080/10903120390936554. PMID: 12879385. Exclusion: KQ4 Triage.

92. Cabrini L, Landoni G, Baiardo Redaelli M, et al. Tracheal intubation in critically ill patients: a comprehensive systematic review of randomized trials. Crit Care. 2018 Jan 20;22(1):6. doi: 10.1186/s13054-017-19273. PMID: 29351759. Exclusion: Ineligible population.

93. Cady CE, Weaver MD, Pirrallo RG, et al. Effect of emergency medical technicianplaced Combitubes on outcomes after outof-hospital cardiopulmonary arrest. Prehosp Emerg Care. 2009 Oct-Dec;13(4):495-9. doi: 10.1080/10903120903144874. PMID: 19731162. Exclusion: Ineligible comparison or no comparison.

94. Cai AR, Hodgman EI, Kumar PB, et al. Evaluating pre burn center intubation practices: an update. J Burn Care Res. 2017 Jan/Feb;38(1):e23-e9. doi: 10.1097/BCR.0000000000000457. PMID: 27775982. Exclusion: Ineligible comparison or no comparison.

95. Calkins TR, Miller K, Langdorf MI. Success and complication rates with prehospital placement of an esophageal-tracheal combitube as a rescue airway. Prehospital Disaster Med. 2006 Mar-Apr;21(2):97-100. doi: 10.1017/s1049023x00003423. PMID: 16770999. Exclusion: Ineligible comparison or no comparison.

96. Cardiology ESo. Bag-mask ventilation appears less safe than endotracheal intubation in cardiac arrest 2017 Aug 27. Exclusion: Ineligible study design.

97. Carlson JN, Hostler D, Guyette FX, et al. Derivation and validation of the Prehospital Difficult Airway IdentificationTool (PreDAIT): a predictive model for difficult intubation. West J Emerg Med. 2017 Jun;18(4):662-72. doi: 10.5811/westjem.2017.1.32938. PMID: 28611887. Exclusion: Ineligible outcome.
98. Carlson JN, Quintero J, Guyette FX, et al. Variables associated with successful intubation attempts using video laryngoscopy: a preliminary report in a helicopter emergency medical service. Prehosp Emerg Care. 2012 AprJun;16(2):293-8. doi: 10.3109/10903127.2011.640764. PMID: 22191806. Exclusion: Ineligible comparison or no comparison.

99. Carlson JN, Wang HE. Updates in emergency airway management. Curr Opin Crit Care. 2018 Dec;24(6):525-30. doi: 10.1097/MCC.0000000000000552. PMID: 30239412. Exclusion: Ineligible publication type.

100. Carr BG, Brachet T, David G, et al. The time cost of prehospital intubation and intravenous access in trauma patients. Prehosp Emerg Care. 2008 JulSep;12(3):327-32. doi: 10.1080/10903120802096928. PMID: 18584500. Exclusion: Ineligible outcome.

101. Castle N. Qualitative interview study of clinicians' opinions of which intubation aids to use following a CBRN incident. Journal of Paramedic Practice. 2012;4(4):226-34. Exclusion: Ineligible outcome.

102. Caulfield EV, Dutton RP, Floccare DJ, et al. Prehospital hypocapnia and poor outcome after severe traumatic brain injury. J Trauma. 2009 Jun;66(6):1577-82; discussion 83. doi: https://dx.doi.org/10.1097/TA.0b013e3181a 3931d. PMID: 19509617. Exclusion: Ineligible comparison or no comparison.

103. Cavus E, Janssen S, Reifferscheid F, et al. Videolaryngoscopy for physician-based, prehospital emergency intubation: a prospective, randomized, multicenter comparison of different blade types using A.P. Advance, C-MAC System, and KingVision. Anesth Analg. 2018 May;126(5):1565-74. doi: 10.1213/ANE.0000000000002735. PMID: 29239965. Exclusion: KQ4 Triage.

104. Cavus E, Janssen S, Reifferscheid F, et al. A comparison of different video laryngoscopes for prehospital emergency intubation: a prospective, randomized multicenter study. Notarzt. 2019;35(1):23-31. Exclusion: Not in English, but may be relevant. 
105. Chastel B, Perrier V, Germain A, et al. Usefulness of the Airtraq DLTM videolaryngoscope for placing a doublelumen tube. Anaesth Crit Care Pain Med. 2015 Apr;34(2):89-93. doi: 10.1016/j.accpm.2014.06.004. PMID: 25858618. Exclusion: Ineligible setting.

106. Chen X, Gestring ML, Rosengart MR, et al. Speed is not everything: identifying patients who may benefit from helicopter transport despite faster ground transport. J Trauma Acute Care Surg. 2018 Apr;84(4):549-57. doi: 10.1097/TA.0000000000001769. PMID: 29251708. Exclusion: Ineligible comparison or no comparison.

107. Chenaitia H, Soulleihet V, Massa H, et al. The easytube for airway management in prehospital emergency medicine. Resuscitation. 2010 Nov;81(11):1516-20. doi: 10.1016/j.resuscitation.2010.06.002. PMID: 20621409. Exclusion: Ineligible comparison or no comparison.

108. Chiumello D, Coppola S, Froio S, et al. Noninvasive ventilation in chest trauma: systematic review and meta-analysis. Intensive Care Med. 2013 Jul;39(7):117180. doi: 10.1007/s00134-013-2901-4. PMID: 23571872. Exclusion: Ineligible intervention.

109. Cho J, Cho YS, You JS, et al. Current status of emergency airway management for elderly patients in Korea: multicentre study using the Korean Emergency Airway Management Registry. Emerg Med Australas. 2013 Oct;25(5):439-44. doi: 10.1111/1742-6723.12122. PMID: 24099373. Exclusion: Studies that meet other criteria but setting is in Emergency department.

110. Cho YS, Cho J, Chung HS, et al. Assessment of emergency airway management techniques in Korea using an online registration system: a multicenter study. J Emerg Med. 2015 Jan;48(1):1-9. doi: 10.1016/j.jemermed.2014.06.044. PMID: 25271178. Exclusion: Studies that meet other criteria but setting is in Emergency department.

111. Choffat C, Delhumeau C, Fournier N, et al. Effect of pre-hospital intubation in patients with severe traumatic brain injury on outcome: a prospective cohort study. J Clin Med. 2019 Apr 06;8(4):06. doi: 10.3390/jcm8040470. PMID: 30959868. Exclusion: Ineligible comparison or no comparison.
112. Chou D, Harada MY, Barmparas G, et al. Field intubation in civilian patients with hemorrhagic shock is associated with higher mortality. J Trauma Acute Care Surg. 2016 Feb;80(2):278-82. doi: 10.1097/TA.0000000000000901. PMID: 26491803. Exclusion: Ineligible comparison or no comparison.

113. Chu H-L, Chiang W-C, Hsu S-H, et al. Association of prehospital endotracheal intubation with survival of patients with outof-hospital cardiac arrest: a 5-year retrospective cohort study. Resuscitation. 2015;96:7-. Exclusion: Ineligible comparison or no comparison.

114. Cierniak M, Timler D, Sobczak R, et al. Analysis of the incidence of postintubation injuries in patients intubated in the prehospital or early hospital conditions of the hospital emergency department and the intensive care unit. Ther Clin Risk Manag. 2015;11:1489-96. doi:

10.2147/TCRM.S90181. PMID: 26491335. Exclusion: Ineligible setting.

115. Cobas MA, De la Pena MA, Manning R, et al. Prehospital intubations and mortality: a level 1 trauma center perspective. Anesth Analg. 2009 Aug;109(2):489-93. doi: 10.1213/ane.0b013e3181aa3063. PMID: 19608824. Exclusion: Ineligible outcome.

116. Colak A, Copuroglu E, Yilmaz A, et al. A comparison of the effects of different types of laryngoscope on the cervical motions: randomized clinical trial. Balkan Med J. 2015 Apr;32(2):176-82. doi: 10.5152/balkanmedj.2015.15335. PMID: 26167342. Exclusion: Ineligible intervention.

117. Colwell CB, McVaney KE, Haukoos JS, et al. An evaluation of out-of-hospital advanced airway management in an urban setting. Acad Emerg Med. 2005 May;12(5):417-22. doi: 10.1197/j.aem.2004.11.024. PMID: 15860695. Exclusion: Ineligible comparison or no comparison.

118. Combes X, Jabre P, Margenet A, et al. Unanticipated difficult airway management in the prehospital emergency setting: prospective validation of an algorithm. Anesthesiology. 2011 Jan;114(1):105-10. doi: 10.1097/ALN.0b013e318201c42e. PMID: 21169803. Exclusion: Ineligible comparison or no comparison. 
119. Cone DC. Are alternative airway devices beneficial in out-of-hospital cardiac arrest? Resuscitation. 2012a Mar;83(3):275-6. doi: 10.1016/j.resuscitation.2011.12.024. PMID: 22210503. Exclusion: Ineligible publication type.

120. Cone DC. Comparing laryngoscopy devices: devices, settings, clinicians - and outcomes. Resuscitation. 2016 Aug;105:A4-5. doi: 10.1016/j.resuscitation.2016.05.009. PMID: 27216563. Exclusion: Ineligible publication type.

121. Cone DC, Middleton PM. ROC, paper, scissors: tracheal intubation or supraglottic airway for out-of-hospital cardiac arrest? Resuscitation. 2012b Sep;83(9):1047-8. doi: 10.1016/j.resuscitation.2012.07.001. PMID: 22789680. Exclusion: Ineligible publication type.

122. Cong ML. Flying doctor emergency airway registry: a 3-year, prospective, observational study of endotracheal intubation by the Queensland Section of the Royal Flying Doctor Service of Australia. Emerg Med J. 2012 Mar;29(3):249-50. doi:

10.1136/emj.2010.100651. PMID:

20844099 Exclusion: Ineligible comparison or no comparison.

123. Cook TM, Hommers C. New airways for resuscitation? Resuscitation. 2006 Jun;69(3):371-87. doi: 10.1016/j.resuscitation.2005.10.015. PMID: 16564123. Exclusion: Ineligible study design.

124. Crewdson K, Fragoso-Iniguez M, Lockey DJ. Requirement for urgent tracheal intubation after traumatic injury: a retrospective analysis of 11,010 patients in the Trauma Audit Research Network database. Anaesthesia. 2019

Sep;74(9):1158-64. doi: 10.1111/anae.14692. PMID: 31069782. Exclusion: Ineligible comparison or no comparison.

125. Crewdson K, Lockey D. Advanced airway management for pre-hospital trauma patients. Trauma. 2016;18(2):111-8. Exclusion: Ineligible publication type.

126. Cross SJ. Airway management and endotracheal intubation by paramedics for cardiac arrest. Journal of Paramedic Practice. 2018;10(1):8-14. Exclusion: Ineligible publication type.
127. Cudnik MT, Newgard CD, Wang H, et al. Endotracheal intubation increases out-ofhospital time in trauma patients. Prehosp Emerg Care. 2007 Apr-Jun;11(2):224-9. doi: 10.1080/10903120701205208. PMID: 17454813. Exclusion: Ineligible outcome.

128. Cudnik MT, Newgard CD, Wang H, et al. Distance impacts mortality in trauma patients with an intubation attempt. Prehosp Emerg Care. 2008 Oct-Dec;12(4):459-66. doi: 10.1080/10903120802290745. PMID: 18924009. Exclusion: Ineligible comparison or no comparison.

129. Cushman JT, Zachary Hettinger A, Farney A, et al. Effect of intensive physician oversight on a prehospital rapid-sequence intubation program. Prehosp Emerg Care. 2010 Jul-Sep;14(3):310-6. doi: 10.3109/10903121003760200. PMID: 20397866. Exclusion: KQ4 Triage.

130. D'Anza B, Knight J, Greene JS. Does body mass index predict tracheal airway size? Laryngoscope. 2015 May;125(5):1093-7. doi: 10.1002/lary.24943. PMID: 25251641. Exclusion: Ineligible intervention.

131. Dargin J, Medzon R. Emergency department management of the airway in obese adults. Ann Emerg Med. 2010 Aug;56(2):95-104. doi: 10.1016/j.annemergmed.2010.03.011. PMID: 20363528. Exclusion: Ineligible publication type.

132. Dargin JM, Emlet LL, Guyette FX. The effect of body mass index on intubation success rates and complications during emergency airway management. Intern Emerg Med. 2013 Feb;8(1):75-82. doi: 10.1007/s11739-012-0874-x. PMID: 23184440. Exclusion: Studies that meet other criteria but setting is in Emergency department.

133. Davis DP. Early ventilation in traumatic brain injury. Resuscitation. 2008a Mar;76(3):333-40. doi: 10.1016/j.resuscitation.2007.08.004. PMID: 17870227. Exclusion: Ineligible publication type.

134. Davis DP. Prehospital intubation of braininjured patients. Curr Opin Crit Care. 2008b Apr;14(2):142-8. doi: 10.1097/MCC.0b013e3282f63c40. PMID: 18388675. Exclusion: Systematic review used to identify primary studies. 
135. Davis DP, Fisher R, Buono C, et al. Predictors of intubation success and therapeutic value of paramedic airway management in a large, urban EMS system. Prehosp Emerg Care. 2006a Jul-

Sep;10(3):356-62. doi: 10.1080/10903120600725751. PMID: 16801280. Exclusion: Ineligible comparison or no comparison.

136. Davis DP, Heister R, Poste JC, et al. Ventilation patterns in patients with severe traumatic brain injury following paramedic rapid sequence intubation. Neurocrit Care. 2005c;2(2):165-71. doi:

10.1385/NCC:2:2:165. PMID: 16159059.

Exclusion: Ineligible comparison or no comparison.

137. Davis DP, Hoyt DB, Ochs M, et al. The effect of paramedic rapid sequence intubation on outcome in patients with severe traumatic brain injury. J Trauma. 2003c Mar;54(3):444-53. doi: 10.1097/01.Ta.0000053396.02126.Cd. PMID: 12634522. Exclusion: Ineligible comparison or no comparison.

138. Davis DP, Idris AH, Sise MJ, et al. Early ventilation and outcome in patients with moderate to severe traumatic brain injury. Crit Care Med. 2006b Apr;34(4):1202-8. doi: 10.1097/01.CCM.0000208359.74623.1C. PMID: 16484927. Exclusion: Ineligible comparison or no comparison.

139. Davis DP, Kimbro TA, Vilke GM. The use of midazolam for prehospital rapid-sequence intubation may be associated with a doserelated increase in hypotension. Prehosp Emerg Care. 2001 Apr-Jun;5(2):163-8. doi: 10.1080/10903120190940065. PMID: 11339727. Exclusion: Ineligible comparison or no comparison.

140. Davis DP, Koprowicz KM, Newgard CD, et al. The relationship between out-of-hospital airway management and outcome among trauma patients with Glasgow Coma Scale Scores of 8 or less. Prehosp Emerg Care. 2011 Apr-Jun;15(2):184-92. doi: 10.3109/10903127.2010.545473. PMID: 21309705. Exclusion: Ineligible comparison or no comparison.

141. Davis DP, Lemieux J, Serra J, et al. Preoxygenation reduces desaturation events and improves intubation success. Air Med J. 2015 Mar-Apr;34(2):82-5. doi: 10.1016/j.amj.2014.12.007. PMID: 25733113. Exclusion: KQ4 Triage.
142. Davis DP, Ochs M, Hoyt DB, et al. Paramedic-administered neuromuscular blockade improves prehospital intubation success in severely head-injured patients. J Trauma. 2003b Oct;55(4):713-9. doi: 10.1097/01.TA.0000037428.65987.12. PMID: 14566128. Exclusion: Ineligible comparison or no comparison.

143. Davis DP, Olvera DJ. HEAVEN criteria: derivation of a new difficult airway prediction tool. Air Med J. 2017 Jul Aug;36(4):195-7. doi: 10.1016/j.amj.2017.04.001. PMID: 28739243. Exclusion: Ineligible comparison or no comparison.

144. Davis DP, Peay J, Sise MJ, et al. Prehospital airway and ventilation management: a trauma score and injury severity score-based analysis. J Trauma. 2010 Aug;69(2):294301. doi: 10.1097/TA.0b013e3181dc6c7f. PMID: 20699737. Exclusion: Ineligible comparison or no comparison.

145. Davis DP, Peay J, Sise MJ, et al. The impact of prehospital endotracheal intubation on outcome in moderate to severe traumatic brain injury. J Trauma. 2005b May;58(5):933-9. doi: 10.1097/01.ta.0000162731.53812.58. PMID: 15920406. Exclusion: Ineligible comparison or no comparison.

146. Davis DP, Stern J, Sise MJ, et al. A followup analysis of factors associated with headinjury mortality after paramedic rapid sequence intubation. J Trauma. 2005a Aug;59(2):486-90. doi: 10.1097/00005373200508000-00037. PMID: 16294099. Exclusion: KQ4 Triage.

147. Davis DP, Valentine C, Ochs M, et al. The Combitube as a salvage airway device for paramedic rapid sequence intubation. Ann Emerg Med. 2003a Nov;42(5):697-704. doi: 10.1016/s0196-0644(03)00396-2. PMID: 14581924. Exclusion: Ineligible comparison or no comparison.

148. Davis PR, Rickards AC, Ollerton JE. Determining the composition and benefit of the pre-hospital medical response team in the conflict setting. J R Army Med Corps. 2007 Dec;153(4):269-73. doi: 10.1136/jramc-153-04-10. PMID: 18619161. Exclusion: Ineligible publication type. 
149. de Caen AR, Kleinman ME, Chameides L, et al. Part 10: paediatric basic and advanced life support: 2010 International Consensus on Cardiopulmonary Resuscitation and Emergency Cardiovascular Care Science with Treatment Recommendations. Resuscitation. 2010 Oct;81 Suppl 1:e213-59. doi: 10.1016/j.resuscitation.2010.08.028. PMID: 20956041. Exclusion: Ineligible publication type.

150. De Lorenzo RA, Abbott CA. Effect of a focused and directed continuing education program on prehospital skill maintenance in key resuscitation areas. J Emerg Med. 2007 Oct;33(3):293-7. doi: 10.1016/j.jemermed.2007.02.060. PMID: 17976562. Exclusion: Ineligible outcome.

151. Deakin CD, Clarke T, Nolan J, et al. A critical reassessment of ambulance service airway management in prehospital care: Joint Royal Colleges Ambulance Liaison Committee Airway Working Group, June 2008. Emerg Med J. 2010 Mar;27(3):22633. doi: 10.1136/emj.2009.082115. PMID: 20304897. Exclusion: Ineligible publication type.

152. Denninghoff KR, Griffin MJ, Bartolucci AA, et al. Emergent endotracheal intubation and mortality in traumatic brain injury. West J Emerg Med. 2008 Nov;9(4):184-9. PMID: 19561742. Exclusion: Ineligible population.

153. Denninghoff KR, Nuno T, Pauls Q, et al. Prehospital intubation is associated with favorable outcomes and lower mortality in ProTECT III. Prehosp Emerg Care. 2017 Sep-Oct;21(5):539-44. doi: 10.1080/10903127.2017.1315201. PMID: 28489506. Exclusion: KQ4 Triage.

154. Denver Metro Airway Study G. A prospective multicenter evaluation of prehospital airway management performance in a large metropolitan region. Prehosp Emerg Care. 2009 JulSep;13(3):304-10. doi: 10.1080/10903120902935280. PMID: 19499465. Exclusion: Ineligible comparison or no comparison.

155. Devitt JH, Brooks DA, Oakley PA, et al. Mask lung ventilation by ambulance personnel: a performance assessment. Can J Anaesth. 1994 Feb;41(2):111-5. doi: 10.1007/BF03009802. PMID: 8131225. Exclusion: Ineligible population.
156. Dietrich SK, Mixon MA, Rogoszewski RJ, et al. Hemodynamic effects of propofol for induction of rapid sequence intubation in traumatically injured patients. Am Surg. 2018 Sep 01;84(9):1504-8. PMID: 30268185. Exclusion: Studies that meet other criteria but setting is in Emergency department.

157. Diggs LA, Viswakula SD, Sheth-Chandra $\mathrm{M}$, et al. A pilot model for predicting the success of prehospital endotracheal intubation. Am J Emerg Med. 2015b Feb;33(2):202-8. doi: 10.1016/j.ajem.2014.11.020. PMID: 25488339. Exclusion: KQ4 Triage.

158. DiRusso SM, Sullivan T, Risucci D, et al. Intubation of pediatric trauma patients in the field: predictor of negative outcome despite risk stratification. J Trauma. 2005 Jul;59(1):84-90; discussion -1. doi: 10.1097/01.ta.0000171462.28379.f3. PMID: 16096544. Exclusion: Ineligible outcome.

159. Donald C, Duncan R, Thakore S. Predictors of the need for rapid sequence intubation in the poisoned patient with reduced Glasgow coma score. Emerg Med J. 2009 Jul;26(7):510-2. doi: 10.1136/emj.2008.064998. PMID: 19546273. Exclusion: Studies that meet other criteria but setting is in Emergency department.

160. Donoghue A, Hsieh TC, Nishisaki A, et al. Tracheal intubation during pediatric cardiopulmonary resuscitation: a videography-based assessment in an emergency department resuscitation room. Resuscitation. 2016 Feb;99:38-43. doi: 10.1016/j.resuscitation.2015.11.019. PMID: 26703462. Exclusion: Ineligible study design.

161. Doran JV, Tortella BJ, Drivet WJ, et al. Factors influencing successful intubation in the prehospital setting. Prehospital Disaster Med. 1995 Oct-Dec;10(4):259-64. doi: 10.1017/s1049023x00042138. PMID: 10155438. Exclusion: Ineligible comparison or no comparison.

162. Dorges V, Ocker H, Hagelberg S, et al. Smaller tidal volumes with room-air are not sufficient to ensure adequate oxygenation during bag-valve-mask ventilation. Resuscitation. 2000b Mar;44(1):37-41. doi: 10.1016/s0300-9572(99)00161-6. PMID: 10699698. Exclusion: Ineligible comparison or no comparison. 
163. Dorges V, Ocker H, Hagelberg S, et al. Optimisation of tidal volumes given with self-inflatable bags without additional oxygen. Resuscitation. 2000a Feb;43(3):195-9. doi: 10.1016/s03009572(99)00148-3. PMID: 10711488. Exclusion: Ineligible comparison or no comparison.

164. Douglas Evans CC, Brison RJ, Howes D, et al. Prehospital non-drug assisted intubation for adulttrauma patients with a Glasgow Coma Score less than 9. Emerg Med J. 2013 Nov 30(11):935-41. doi: 10.1136/emermed2012-201578. PMID: 23144080 Exclusion: Ineligible intervention.

165. Drayna PC, Browne LR, Guse CE, et al. Prehospital pediatric care: opportunities for training, treatment, and research. Prehosp Emerg Care. 2015 Jul-Sep;19(3):441-7. doi: 10.3109/10903127.2014.995850. PMID:

25658967. Exclusion: Ineligible comparison or no comparison.

166. Driver BE, Klein LR, Prekker ME, et al. Drug order in rapid sequence intubation. Acad Emerg Med. 2019 Sep;26(9):1014-21. doi: 10.1111/acem.13723. PMID: 30834639. Exclusion: Studies that meet other criteria but setting is in Emergency department.

167. Duby R, Hansen M, Meckler G, et al. Safety events in high risk prehospital neonatal calls. Prehosp Emerg Care. 2018 JanFeb;22(1):34-40. doi: 10.1080/10903127.2017.1347222. PMID: 28857641. Exclusion: Ineligible comparison or no comparison.

168. Duchatelet C, Kalmar AF, Monsieurs KG, et al. Chest compressions during ventilation in out-of-hospital cardiac arrest cause reversed airflow. Resuscitation. 2018 Aug;129:97102. doi: 10.1016/j.resuscitation.2018.03.035. PMID: 29604395. Exclusion: Ineligible intervention.

169. Duchynski R, Brauer K, Hutton K, et al. The quick look airway classification. A useful tool in predicting the difficult out-ofhospital intubation: experience in an air medical transport program. Air Med J. 1998 Apr-Jun;17(2):46-50. doi: 10.1016/s1067991x(98)90019-0. PMID: 10180783. Exclusion: Ineligible comparison or no comparison.
170. Duggan LV, Lockhart SL, Cook TM, et al. The Airway App: exploring the role of smartphone technology to capture emergency front-of-neck airway experiences internationally. Anaesthesia. 2018 Jun;73(6):703-10. doi: 10.1111/anae.14247. PMID: 29533465. Exclusion: Ineligible intervention.

171. Dumas G, Chevret S, Le Corre M, et al. Typology of published randomized controlled trials investigating initial ventilation strategy in critically ill patients with acute respiratory failure: a methodologic review. Chest. 2020 Sep;158(3):986-98. doi: 10.1016/j.chest.2020.03.082. PMID: 32387523. Exclusion: Ineligible setting, Ineligible population.

172. Duong HV, Herrera LN, Moore JX, et al. National characteristics of emergency medical services responses for older adults in the United States. Prehosp Emerg Care. 2018 Jan-Feb;22(1):7-14. doi: 10.1080/10903127.2017.1347223. PMID: 28862480. Exclusion: Ineligible outcome.

173. Dupanovic M, Fox H, Kovac A. Management of the airway in multitrauma. Curr Opin Anaesthesiol. 2010 Apr;23(2):276-82. doi: 10.1097/ACO.0b013e3283360b4f. PMID: 20042974. Exclusion: Ineligible publication type.

174. Dyson K, Bray J, Smith K, et al. A systematic review of the effect of emergency medical service practitioners' experience and exposure to out-of-hospital cardiac arrest on patient survival and procedural performance. Resuscitation. 2014 Sep;85(9):1134-41. doi: 10.1016/j.resuscitation.2014.05.020. PMID: 24892269. Exclusion: Systematic review used to identify primary studies.

175. Eckert MJ, Davis KA, Reed RL, 2nd, et al. Ventilator-associated pneumonia, like real estate: location really matters. J Trauma. 2006b Jan;60(1):104-10; discussion 10. doi: 10.1097/01.ta.0000197376.98296.7c. PMID: 16456443. Exclusion: Ineligible outcome.

176. Eckert MJ, Wade TE, Davis KA, et al. Ventilator-associated pneumonia after combined burn and trauma is caused by associated injuries and not the burn wound. J Burn Care Res. 2006a Jul-Aug;27(4):45762. doi: 10.1097/01.BCR.0000226034.84068.CF. PMID: 16819348. Exclusion: Ineligible population. 
177. Edwards T. Does prehospital intubation make a difference to survival rates in out-ofhospital cardiac arrest? Journal of Paramedic Practice. 2011;3(12):709-. Exclusion: Ineligible publication type.

178. Egly J, Custodio D, Bishop N, et al. Assessing the impact of prehospital intubation on survival in out-of-hospital cardiac arrest. Prehosp Emerg Care. 2011 Jan-Mar;15(1):44-9. doi: 10.3109/10903127.2010.514090. PMID: 20858132. Exclusion: Ineligible comparison or no comparison.

179. Ehrlich PF, Seidman PS, Atallah O, et al. Endotracheal intubations in rural pediatric trauma patients. J Pediatr Surg. 2004 Sep;39(9):1376-80. doi: 10.1016/j.jpedsurg.2004.05.010. PMID: 15359393. Exclusion: Ineligible comparison or no comparison.

180. Eismann H, Sieg L, Otten O, et al. Impact of the laryngeal tube as supraglottic airway device on blood flow of the internal carotid artery in patients undergoing general anaesthesia. Resuscitation. 2019

May;138:141-5. doi:

10.1016/j.resuscitation.2019.03.010. PMID: 30885823 .

181. El-Ganzouri AR, Marzouk S, Abdelalem N, et al. Blind versus fiberoptic laryngoscopic intubation through air Q laryngeal mask airway. Egyptian journal of anaesthesia. 2011;27(4):213-8. doi: 10.1016/j.egja.2011.07.001. Exclusion: Ineligible setting.

182. Elmer J, Brown F, Martin-Gill C, et al. Prevalence and predictors of post-intubation hypotension in prehospital trauma care. Prehosp Emerg Care. 2019 Oct 22:1-9. doi: 10.1080/10903127.2019.1670300. PMID: 31566990. Exclusion: Ineligible comparison or no comparison.

183. El-Tahan MR, El Kenany S, Khidr AM, et al. Cervical spine motion during tracheal intubation with King VisionTM video laryngoscopy and conventional laryngoscopy: a crossover randomized study. Minerva Anestesiol. 2017

Nov;83(11):1152-60. doi: 10.23736/S03759393.17.11913-9. PMID: 28607336.

Exclusion: Ineligible setting.
184. Emami P, Czorlich P, Fritzsche FS, et al. Observed versus expected mortality in pediatric patients intubated in the field with Glasgow Coma Scale scores $<9$. Eur J Trauma Emerg Surg. 2019 Oct;45(5):76976. doi: 10.1007/s00068-018-01065-2. PMID: 30631886. Exclusion: Ineligible comparison or no comparison.

185. Erler CJ, Rutherford WF, Rodman G, et al. Inadequate respiratory support in head injury patients. Air Med J. 1993;12(7):2236. Exclusion: Ineligible comparison or no comparison.

186. Espino-Nunez JS, Quinto-Sanchez M, Carrada-Varela AC, et al. Physician prehospital care in Mexico City: retrospective analysis of endotracheal intubation in patients with severe head trauma. Prehospital Disaster Med. 2020 Apr;35(2):128-32. doi: 10.1017/S1049023X20000035. PMID: 31973785. Exclusion: Ineligible comparison or no comparison, Ineligible intervention.

187. Etchill EW, Myers SP, McDaniel LM, et al. Should all massively transfused patients be treated equally? An analysis of massive transfusion ratios in the nontrauma setting. Crit Care Med. 2017 Aug;45(8):1311-6. doi: 10.1097/CCM.0000000000002498. PMID: 28537938. Exclusion: Ineligible setting.

188. Evans CC, Brison RJ, Howes D, et al. Prehospital non-drug assisted intubation for adult trauma patients with a Glasgow Coma Score less than 9. Emerg Med J. 2013 Nov;30(11):935-41. doi: 10.1136/emermed2012-201578. PMID: 23144080. Exclusion: Ineligible intervention.

189. Evans HL, Warner K, Bulger EM, et al. Prehospital intubation factors and pneumonia in trauma patients. Surg Infect (Larchmt). 2011 Oct;12(5):339-44. doi:

10.1089/sur.2010.074. PMID: 21933010. Exclusion: Ineligible outcome.

190. Fakhry SM, Scanlon JM, Robinson L, et al. Prehospital rapid sequence intubation for head trauma: conditions for a successful program. J Trauma. 2006 May;60(5):9971001. doi: 10.1097/01.ta.0000217285.94057.5e. PMID: 16688061. Exclusion: Ineligible comparison or no comparison.

191. Falcone RE, Herron H, Dean B, et al. Emergency scene endotracheal intubation before and after the introduction of a rapid sequence induction protocol. Air Med J. 1996 Oct-Dec;15(4):163-7. doi: 10.1016/s1067-991x(96)90024-3. PMID: 10162102. Exclusion: Ineligible outcome. 
192. Fan G, Diao B, Zhang Y. Application of modified oropharyngeal airway in emergency care of patients with traumatic brain injury. International medical journal. 2014;21(2):163-5. Exclusion: Ineligible setting.

193. Fan YJ, Dai CY, Huang DC, et al. Does tracheal intubation really matter? Discrepant survival between laryngeal mask and endotracheal intubation during out-ofhospital cardiac arrest. J Formos Med Assoc. 2017 Feb;116(2):134-5. doi: 10.1016/j.jfma.2016.11.001. PMID: 28003114. Exclusion: Ineligible publication type.

194. Fawcett VJ, Warner KJ, Cuschieri J, et al. Pre-hospital aspiration is associated with increased pulmonary complications. Surg Infect (Larchmt). 2015 Apr;16(2):159-64. doi: 10.1089/sur.2013.237. PMID: 24896218. Exclusion: Ineligible intervention.

195. Fitzgerald RD, Krafft P, Skrbensky G, et al. Excursions of the cervical spine during tracheal intubation: blind oral intubation compared with direct laryngoscopy. Anaesthesia. 1994 Feb;49(2):111-5. doi: 10.1111/j.1365-2044.1994.tb03364.x. PMID: 8093140. Exclusion: Ineligible setting.

196. Flavell E, Boyle MJ. Which is more effective for ventilation in the prehospital setting during cardiopulmonary resuscitation, the laryngeal mask airway or the bag-valve-mask? -- A review of the literature. Journal of Emergency Primary Health Care. 2010;8(3):1-10. Exclusion: Systematic review used to identify primary studies.

197. Fok PT, Teubner D, Purdell-Lewis J, et al. Predictors of prehospital on-scene time in an Australian emergency retrieval service. Prehospital Disaster Med. 2019 Jun;34(3):317-21. doi: 10.1017/S1049023X19004394. PMID: 31204644. Exclusion: Ineligible outcome.

198. Fouche PF, Simpson PM, Bendall J, et al. Airways in out-of-hospital cardiac arrest: systematic review and meta-analysis. Prehosp Emerg Care. 2014 AprJun;18(2):244-56. doi: 10.3109/10903127.2013.831509. PMID: 24111481. Exclusion: Systematic review used to identify primary studies.
199. Fouche PF, Stein C, Jennings PA, et al. Review article: emergency endotracheal intubation in non-traumatic brain pathologies: a systematic review and metaanalysis. Emerg Med Australas. 2019 Aug;31(4):533-41. doi: 10.1111/17426723.13304. PMID: 31041848 . Exclusion: Systematic review used to identify primary studies.

200. Fouche PF, Stein C, Simpson P, et al. Nonphysician out-of-hospital rapid sequence intubation success and adverse events: a systematic review and meta-analysis. Ann Emerg Med. 2017 Oct;70(4):449-59. e20. doi: 10.1016/j.annemergmed.2017.03.026. PMID: 28559038. Exclusion: Ineligible comparison or no comparison.

201. Fouche PF, Stein C, Simpson P, et al. Flight versus ground out-of-hospital rapid sequence intubation success: a systematic review and meta-analysis. Prehosp Emerg Care. 2018 Sep-Oct;22(5):578-87. doi: 10.1080/10903127.2017.1423139. PMID: 29377753. Exclusion: Systematic review used to identify primary studies.

202. Fowler RL, Leba C, Mehkri F, et al. The BVM Effect. An overview of studies assessing airway management in out-ofhospital cardiac arrest. J Emerg Med Serv JEMS. 2015 Oct;40(10):58-61, 7. PMID: 26669069. Exclusion: Ineligible publication type.

203. Frankel H, Rozycki G, Champion H, et al. The use of TRISS methodology to validate prehospital intubation by urban EMS providers. Am J Emerg Med. 1997 Nov;15(7):630-2. doi: 10.1016/s07356757(97)90174-1. PMID: 9375541. Exclusion: Ineligible comparison or no comparison.

204. Franschman G, Peerdeman SM, Greuters S, et al. Prehospital endotracheal intubation in patients with severe traumatic brain injury: guidelines versus reality. Resuscitation. 2009 Oct; $80(10): 1147-51$. doi: 10.1016/j.resuscitation.2009.06.029. PMID: 19632024. Exclusion: Ineligible comparison or no comparison.

205. Frascone RJ, Wewerka SS, Burnett AM, et al. Supraglottic airway device use as a primary airway during rapid sequence intubation. Air Med J. 2013 MarApr;32(2):93-7. doi: 10.1016/j.amj.2012.06.008. PMID: 23452368. Exclusion: Ineligible comparison or no comparison. 
206. Fukuda T, Ohashi-Fukuda N, Kondo Y, et al. Epidemiology, risk factors, and outcomes of out-of-hospital cardiac arrest caused by stroke: a population-based study. Medicine (Baltimore). 2016 Apr;95(14):e3107. doi: 10.1097/MD.0000000000003107. PMID: 27057834. Exclusion: Ineligible intervention.

207. Fuller BM, Ferguson IT, Mohr NM, et al. A quasi-experimental, before-after trial examining the impact of an emergency department mechanical ventilator protocol on clinical outcomes and lung-protective ventilation in acute respiratory distress syndrome. Crit Care Med. 2017 Apr;45(4):645-52. doi: 10.1097/CCM.0000000000002268. PMID: 28157140. Exclusion: Ineligible intervention.

208. Fullerton JN, Roberts KJ, Wyse M. Can experienced paramedics perform tracheal intubation at cardiac arrests? Five years experience of a regional air ambulance service in the UK. Resuscitation. 2009 Dec;80(12):1342-5. doi: 10.1016/j.resuscitation.2009.07.023. PMID: 19733428. Exclusion: KQ4 Triage.

209. Fullerton JN, Roberts KJ, Wyse M. Should non-anaesthetists perform pre-hospital rapid sequence induction? An observational study. Emerg Med J. 2011 May;28(5):428-31. doi: 10.1136/emj.2009.086645. PMID: 20660897. Exclusion: KQ4 Triage.

210. Gaither JB, Stolz U, Ennis J, et al. Association between difficult airway predictors and failed prehosptial endotracheal intubation. Air Med J. 2015 Nov-Dec;34(6):343-7. doi: 10.1016/j.amj.2015.06.001. PMID: 26611221. Exclusion: Ineligible comparison or no comparison.

211. Garner AA, Bennett N, Weatherall A, et al. Success and complications by team composition for prehospital paediatric intubation: a systematic review and metaanalysis. Crit Care. 2020 Apr 15;24(1):149. doi: 10.1186/s13054-020-02865-y. PMID: 32295610. Exclusion: Systematic review used to identify primary studies.

212. Garza AG, Gratton MC, Coontz D, et al. Effect of paramedic experience on orotracheal intubation success rates. J Emerg Med. 2003 Oct;25(3):251-6. doi: 10.1016/s0736-4679(03)00198-7. PMID: 14585451. Exclusion: KQ4 Triage.
213. Gasler M, Ruppert M, Lefering R, et al. Prehospital emergent intubation in trauma patients: the influence of etomidate on mortality, morbidity and healthcare resource utilization. Scand J Trauma Resusc Emerg Med. 2019 Jun 07;27(1):61. doi: 10.1186/s13049-019-0637-z. PMID: 31174573. Exclusion: KQ4 Triage.

214. Gende G, Xing Y, Wen J, et al. Management of difficult airways in surgical patients at the Port Moresby General Hospital operating theatre and intensive care unit. P N G Med J. 2012 Mar-Dec;55(1-4):67-75. PMID: 25340215. Exclusion: Ineligible setting.

215. Gercek E, Wahlen BM, Rommens PM. In vivo ultrasound real-time motion of the cervical spine during intubation under manual in-line stabilization: a comparison of intubation methods. Eur J Anaesthesiol. 2008 Jan;25(1):29-36. doi: 10.1017/S0265021507001044. PMID: 17662163. Exclusion: Ineligible setting.

216. Germann CA, Baumann MR, Kendall KM, et al. Performance of endotracheal intubation and rescue techniques by emergency services personnel in an air medical service. Prehosp Emerg Care. 2009 Jan-Mar;13(1):44-9. doi: 10.1080/10903120802474505. PMID: 19145523. Exclusion: Ineligible setting.

217. Gerritse BM, Draaisma JM, Schalkwijk A, et al. Should EMS-paramedics perform paediatric tracheal intubation in the field? Resuscitation. 2008 Nov;79(2):225-9. doi: 10.1016/j.resuscitation.2008.05.016. PMID: 18684547. Exclusion: KQ4 Triage.

218. Glasheen J, Hooper J, Donohue A, et al. Successful endotracheal intubation following a failed first attempt during aeromedical retrieval. Emerg Med J. 2020 May;37(5):314-8. doi: 10.1136/emermed2019-208425. PMID: 32139514. Exclusion: Ineligible setting.

219. Gnugnoli DM, Shafer K. EMS, field intubation. StatPearls Publishing. 2019 Jan;01:01. PMID: 30855809. Exclusion: Ineligible publication type.

220. Goldik Z, Bornstein J, Eden A, et al. Airway management by physicians wearing antichemical warfare gear: comparison between laryngeal mask airway and endotracheal intubation. Eur J Anaesthesiol. 2002 Mar;19(3):166-9. doi: 10.1017/s0265021502000297. PMID: 12071234. Exclusion: Ineligible population. 
221. Goto Y, Goto T, Hagiwara Y, et al. Techniques and outcomes of emergency airway management in Japan: an analysis of two multicentre prospective observational studies, 2010-2016. Resuscitation. 2017 May;114:14-20. doi: 10.1016/j.resuscitation.2017.02.009. PMID: 28219617. Exclusion: Ineligible population.

222. Gough JE, Thomas SH, Brown LH, et al. Does the ambulance environment adversely affect the ability to perform oral endotracheal intubation? Prehospital Disaster Med. 1996 Apr-Jun;11(2):141-3. doi: 10.1017/s1049023x00042837. PMID: 10159739. Exclusion: Ineligible population.

223. Granfeldt A, Avis SR, Nicholson TC, et al. Advanced airway management during adult cardiac arrest: a systematic review. Resuscitation. 2019 Jun;139:133-43. doi: 10.1016/j.resuscitation.2019.04.003. PMID: 30981882. Exclusion: Systematic review used to identify primary studies.

224. Green J, Robinson M, Pilbery R, et al. Research paramedics' observations regarding the challenges and strategies employed in the implementation of a largescale out-of-hospital randomised trial. British Paramedic Journal. 2020;5(1):26-31. doi: 10.29045/14784726.2020.06.5.1.26. Exclusion: Ineligible publication type.

225. Griesdale DE, Liu D, McKinney J, et al. Glidescope ${ }^{\circledR}$ video-laryngoscopy versus direct laryngoscopy for endotracheal intubation: a systematic review and metaanalysis. Can J Anaesth. 2012 Jan;59(1):4152. doi: 10.1007/s12630-011-9620-5. PMID: 22042705. Exclusion: Ineligible setting.

226. Gruber E, Oberhammer R, Balkenhol K, et al. Basic life support trained nurses ventilate more efficiently with laryngeal mask supreme than with facemask or laryngeal tube suction-disposable--a prospective, randomized clinical trial. Resuscitation. 2014 Apr;85(4):499-502. doi: 10.1016/j.resuscitation.2014.01.004. PMID: 24440666. Exclusion: Ineligible intervention.

227. Guay J, Lortie L. An evaluation of pediatric in-hospital advanced life support interventions using the pediatric Utstein guidelines: a review of 203 cardiorespiratory arrests. Can J Anaesth. 2004 Apr;51(4):3738. doi: 10.1007/bf03018242. PMID: 15064267. Exclusion: Ineligible setting.
228. Guihard B, Chollet-Xémard C, Lakhnati P, et al. Effect of rocuronium vs succinylcholine on endotracheal intubation success rate among patients undergoing outof-hospital rapid sequence intubation: a randomized clinical trial. JAMA. 2019;322(23):2303-12. doi: 10.1001/jama.2019.18254. Exclusion: KQ4 Triage.

229. Guyette FX, Farrell K, Carlson JN, et al. Comparison of video laryngoscopy and direct laryngoscopy in a critical care transport service. Prehosp Emerg Care. 2013 Apr-Jun;17(2):149-54. doi: 10.3109/10903127.2012.729128. PMID: 23231426. Exclusion: Ineligible population.

230. Guyette FX, Rittenberger JC, Platt T, et al. Feasibility of basic emergency medical technicians to perform selected advanced life support interventions. Prehosp Emerg Care. 2006 Oct-Dec;10(4):518-21. doi: 10.1080/10903120600726015. PMID: 16997785. Exclusion: Ineligible population.

231. Guyette FX, Wang H, Cole JS. King airway use by air medical providers. Prehosp Emerg Care. 2007b Oct-Dec;11(4):473-6. doi: 10.1080/10903120701536958. PMID: 17907036. Exclusion: Ineligible comparison or no comparison.

232. Haltmeier T, Benjamin E, Siboni S, et al. Prehospital intubation for isolated severe blunt traumatic brain injury: worse outcomes and higher mortality. Eur J Trauma Emerg Surg. 2017 Dec;43(6):731-9. doi: 10.1007/s00068-016-0718-x. PMID: 27567923. Exclusion: Ineligible comparison or no comparison.

233. Haltmeier T, Schnuriger B, Benjamin E, et al. Isolated blunt severe traumatic brain injury in Bern, Switzerland, and the United States: a matched cohort study. J Trauma Acute Care Surg. 2016 Feb;80(2):296-301. doi: 10.1097/TA.0000000000000892. PMID: 26491802. Exclusion: Ineligible comparison or no comparison.

234. Hamilton SA. Prehospital newborn resuscitation. Emerg Med Serv. 1999;28(5):39-44. Exclusion: Ineligible publication type.

235. Hammell CL, Henning JD. Prehospital management of severe traumatic brain injury. BMJ (online). 2009;338(7705):1262. doi: 10.1136/bmj.b1683. PMID: 19454738. Exclusion: Ineligible publication type. 
236. Handly N, Bass RR, New JP, et al. Effect of patient age on airway response by paramedics: frailty or futility? Prehosp Emerg Care. 2011 Jul-Sep;15(3):351-8. doi: 10.3109/10903127.2011.561402. PMID: 21612387. Exclusion: Ineligible outcome.

237. Hansen M, Lambert W, Guise JM, et al. Out-of-hospital pediatric airway management in the United States. Resuscitation. 2015 May;90:104-10. doi: 10.1016/j.resuscitation.2015.02.018. PMID: 25725298. Exclusion: Ineligible comparison or no comparison.

238. Hansen M, Meckler G, Lambert W, et al. Patient safety events in out-of-hospital paediatric airway management: a medical record review by the CSI-EMS. BMJ Open. 2016 Nov 11;6(11):e012259. doi: 10.1136/bmjopen-2016-012259. PMID: 27836871. Exclusion: Ineligible comparison or no comparison.

239. Hardcastle TC, Goff T. Trauma unit emergency doctor airway management. S Afr Med J. 2007 Sep;97(9):864-7. PMID: 17985058. Exclusion: Ineligible comparison or no comparison.

240. Harding C, Hart L. Oxygen titration therapy and hypercapnia risk in COPD. Journal of Paramedic Practice. 2019;11(9):382-8. Exclusion: Ineligible intervention.

241. Harmon MBA, van Meenen DMP, van der Veen A, et al. Practice of mechanical ventilation in cardiac arrest patients and effects of targeted temperature management: a substudy of the targeted temperature management trial. Resuscitation. 2018 Aug; 129:29-36. doi:

10.1016/j.resuscitation.2018.04.040. PMID: 29763713. Exclusion: Ineligible setting.

242. Harris T, Lockey D. Success in physician prehospital rapid sequence intubation: what is the effect of base speciality and length of anaesthetic training? Emerg Med J. 2011 Mar;28(3):225-9. doi:

10.1136/emj.2009.088302. PMID:

21216888. Exclusion: KQ4 Triage.

243. Harrison T, Thomas SH, Wedel SK. Inflight oral endotracheal intubation. Am J Emerg Med. 1997 Oct;15(6):558-61. doi: 10.1016/s0735-6757(97)90156-x. PMID: 9337360. Exclusion: Ineligible comparison or no comparison.
244. Harrison TH, Thomas SH, Wedel SK. Success rates of pediatric intubation by a non-physician-staffed critical care transport service. Pediatr Emerg Care. 2004

Feb;20(2):101-7. doi: 10.1097/01.pec.0000113879.10140.7f. PMID: 14758307. Exclusion: Ineligible outcome.

245. Hartl R, Gerber LM, Iacono L, et al. Direct transport within an organized state trauma system reduces mortality in patients with severe traumatic brain injury. J Trauma. 2006 Jun;60(6):1250-6; discussion 6. doi: 10.1097/01.ta.0000203717.57821.8d. PMID: 16766968. Exclusion: Ineligible intervention.

246. Hartmann T, Krenn CG, Zoeggeler A, et al. The oesophageal-tracheal Combitube Small Adult. Anaesthesia. $2000 \mathrm{Jul} ; 55(7): 670-5$. doi: 10.1046/j.1365-2044.2000.01376.x. PMID: 10919423. Exclusion: Ineligible population.

247. Hasegawa K, Hagiwara Y, Chiba T, et al. Emergency airway management in Japan: interim analysis of a multi-center prospective observational study. Resuscitation. 2012 Apr;83(4):428-33. doi: 10.1016/j.resuscitation.2011.11.027. PMID: 22155701. Exclusion: Ineligible comparison or no comparison.

248. Hashmi NK, Mandel JE, Mirza N. Laryngeal mask airway in laryngoscopies: a safer alternative for the difficult airway. ORL J Otorhinolaryngol Relat Spec. 2009;71(6):342-6. doi: 10.1159/000276990. PMID: 20090393. Exclusion: Ineligible setting.

249. Hawkins RB, Raymond SL, Hamann HC, et al. Outcomes after prehospital endotracheal intubation in suburban/rural pediatric trauma. J Surg Res. 2020 May;249:138-44. doi: 10.1016/j.jss.2019.11.034. PMID: 31954974. Exclusion: Ineligible intervention, Ineligible comparison or no comparison.

250. Haynes BE, Pritting J. A rural emergency medical technician with selected advanced skills. Prehosp Emerg Care. 1999 OctDec;3(4):343-6. doi: 10.1080/10903129908958966. PMID: 10534037. Exclusion: Ineligible comparison or no comparison. 
251. Heegaard WG, Black C, Pasquerella C, et al. Use of the endotracheal tube introducer as an adjunct for oral tracheal intubation in the prehospital setting. Air Med J. 2003 JanFeb;22(1):28-31. doi: 10.1067/mmj.2003.mmj034. PMID: 12522361. Exclusion: Ineligible comparison or no comparison.

252. Heffner AC, Swords D, Kline JA, et al. The frequency and significance of postintubation hypotension during emergency airway management. J Crit Care. 2012

Aug;27(4):417.e9-13. doi: 10.1016/j.jcrc.2011.08.011. PMID: 22033053. Exclusion: Studies that meet other criteria but setting is in Emergency department.

253. Herff H, Paal P, Mitterlechner T, et al. The mouth-to-bag resuscitator during standard anaesthesia induction in apnoeic patients. Resuscitation. 2009 Oct;80(10):1142-6. doi: 10.1016/j.resuscitation.2009.06.026. PMID: 19674827. Exclusion: Ineligible setting.

254. Hernandez MC, Antiel RM, Balakrishnan K, et al. Definitive airway management after prehospital supraglottic rescue airway in pediatric trauma. J Pediatr Surg. 2018 Feb;53(2):352-6. doi: 10.1016/j.jpedsurg.2017.10.004. PMID: 29096887. Exclusion: Ineligible comparison or no comparison.

255. Herrington HC, Weber SM, Andersen PE. Modern management of laryngotracheal stenosis. Laryngoscope. 2006 Sep;116(9):1553-7. doi: 10.1097/01.mlg.0000228006.21941.12. PMID: 16954977. Exclusion: Ineligible intervention.

256. Heschl S, Meadley B, Andrew E, et al. Efficacy of pre-hospital rapid sequence intubation in paediatric traumatic brain injury: a 9-year observational study. Injury. 2018 May;49(5):916-20. doi: 10.1016/j.injury.2018.02.013. PMID: 29452732. Exclusion: Ineligible comparison or no comparison.

257. Hiestand B, Cudnik MT, Thomson D, et al. Rocuronium versus succinylcholine in air medical rapid-sequence intubation. Prehosp Emerg Care. 2011 Oct-Dec;15(4):457-63. doi: 10.3109/10903127.2011.569853. PMID: 21568699. Exclusion: Ineligible population.
258. Hildreth AN, Mejia VA, Maxwell RA, et al. Adrenal suppression following a single dose of etomidate for rapid sequence induction: a prospective randomized study. J Trauma. 2008 Sep;65(3):573-9. doi: 10.1097/TA.0b013e31818255e8. PMID: 18784570. Exclusion: Ineligible setting.

259. Hilton MT, Wayne M, Martin-Gill C. Impact of system-wide King LT airway implementation on orotracheal intubation. Prehosp Emerg Care. 2016 SepOct;20(5):570-7. doi: 10.3109/10903127.2016.1163446. PMID: 27058646. Exclusion: Ineligible outcome.

260. Hinckley WR, Stolz U, Golden AJ, et al. Abstract 4: predictors of definitive airway sans hypoxia/hypotension on first attempt (DASH 1A) success in traumatically injured patients undergoing prehospital intubation...2019 Critical Care Transport Medicine Conference, 15-17 April 2019, Albuquerque, New Mexico. Air Med J. 2019;38(4):259-. PMID: 31539287. Exclusion: Ineligible publication type.

261. Hinkewich C, Green R. The impact of etomidate on mortality in trauma patients. Can J Anaesth. 2014 Jul;61(7):650-5. doi: 10.1007/s12630-014-0161-6. PMID: 24723214. Exclusion: Ineligible setting.

262. Hodgetts TJ, Brown T, Driscoll P, et al. Prehospital cardiac arrest: room for improvement. Resuscitation. 1995 Feb;29(1):47-54. doi: 10.1016/03009572(94)00818-z. PMID: 7784723. Exclusion: Ineligible comparison or no comparison.

263. Hodroge SS, Glenn M, Breyre A, et al. Adult patients with respiratory distress: current evidence-based recommendations for prehospital care. West J Emerg Med. 2020 Jun 25;21(4):849-57. doi: 10.5811/westjem.2020.2.43896. PMID: 32726255. Exclusion: Ineligible intervention., Ineligible publication type.

264. Holmberg TJ, Bowman SM, Warner KJ, et al. The association between obesity and difficult prehospital tracheal intubation. Anesth Analg. 2011 May;112(5):1132-8. doi: 10.1213/ANE.0b013e31820effcc. PMID: 21346165. Exclusion: KQ4 Triage.

265. Holmes J, Peng J, Bair A. Abnormal endtidal carbon dioxide levels on emergency department arrival in adult and pediatric intubated patients. Prehosp Emerg Care. 2012 Apr-Jun;16(2):210-6. doi: 10.3109/10903127.2011.640416. PMID: 22217189. Exclusion: Ineligible comparison or no comparison. 
266. Honold J, Hodrius J, Schwietz T, et al. [Aspiration and pneumonia risk after preclinical invasive resuscitation: Endotracheal intubation and supraglottic airway management with the laryngeal tube S]. Med Klin Intensivmed Notfmed. 2015 Oct;110(7):526-33. doi: 10.1007/s00063015-0018-y. PMID: 25850763. Exclusion: Not in English, but may be relevant.

267. Hooper TJ, De Pasquale M, Strandenes G, et al. Challenges and possibilities in forward resuscitation. Shock. 2014 May;41 Suppl 1:13-20. doi: 10.1097/SHK.0000000000000096. PMID: 24296432. Exclusion: Ineligible comparison or no comparison.

268. Horton CL, Brown CA, 3rd, Raja AS. Trauma airway management. J Emerg Med. 2014 Jun;46(6):814-20. doi: 10.1016/j.jemermed.2013.11.085. PMID: 24582643. Exclusion: Ineligible study design.

269. Howerton D, Watson B. An air medical company's review of crew duty time and the effects on endotracheal intubation success rates...2010 Critical Care Transport Medicine Conference held April 12-14, 2010, San Antonio, Texas. Air Med J. 2010;29(4):159-. Exclusion: Ineligible study design.

270. Hubble MW, Brown L, Wilfong DA, et al. A meta-analysis of prehospital airway control techniques part I: orotracheal and nasotracheal intubation success rates.

Prehosp Emerg Care. 2010a Jul-

Sep;14(3):377-401. doi: 10.3109/10903121003790173. PMID: 20507222. Exclusion: Studies that meet other criteria but setting is in Emergency department.

271. Hubble MW, Tyson C. Impact of early vasopressor administration on neurological outcomes after prolonged out-of-hospital cardiac arrest. Prehospital Disaster Med. 2017 Jun;32(3):297-304. doi: 10.1017/S1049023X17000115. PMID: 28222830. Exclusion: Ineligible outcome.

272. Hubble MW, Wilfong DA, Brown LH, et al. A meta-analysis of prehospital airway control techniques part II: alternative airway devices and cricothyrotomy success rates. Prehosp Emerg Care. 2010b OctDec;14(4):515-30. doi: 10.3109/10903127.2010.497903. PMID: 20809690. Exclusion: Studies that meet other criteria but setting is in Emergency department.
273. Hudson IL, Blackburn MB, Staudt AM, et al. Analysis of casualties that underwent airway management before reaching role 2 facilities in the Afghanistan conflict 20082014. Mil Med. 2020 Jan 07;185(Suppl 1):10-8. doi: $10.1093 / \mathrm{milmed} /$ usz383. PMID: 32074383. Exclusion: Ineligible intervention.

274. Hussmann B, Lefering R, Waydhas C, et al. Prehospital intubation of the moderately injured patient: a cause of morbidity? A matched-pairs analysis of 1,200 patients from the DGU Trauma Registry. Crit Care. 2011;15(5):R207. doi: 10.1186/cc10442. PMID: 21914175. Exclusion: Ineligible comparison or no comparison.

275. Irvin CB, Szpunar S, Cindrich LA, et al. Should trauma patients with a Glasgow Coma Scale score of 3 be intubated prior to hospital arrival? Prehospital Disaster Med. 2010 Nov-Dec;25(6):541-6. doi: 10.1017/s1049023x00008736. PMID: 21181689. Exclusion: Ineligible comparison or no comparison.

276. Izawa J, Iwami T, Gibo K, et al. Timing of advanced airway management by emergency medical services personnel following out-ofhospital cardiac arrest: a population-based cohort study. Resuscitation. 2018 Jul;128:16-23. doi:

10.1016/j.resuscitation.2018.04.024. PMID: 29689354. Exclusion: Ineligible comparison or no comparison.

277. Izawa J, Komukai S, Gibo K, et al. Prehospital advanced airway management for adults with out-of-hospital cardiac arrest: nationwide cohort study. Bmj. 2019 Feb 28;364:1430. doi: 10.1136/bmj.1430. PMID: 30819685. Exclusion: Ineligible comparison or no comparison.

278. Jabre P, Avenel A, Combes X, et al. Morbidity related to emergency endotracheal intubation--a substudy of the KETAmine SEDation trial. Resuscitation. 2011a May;82(5):517-22. doi: 10.1016/j.resuscitation.2011.01.015. PMID: 21345571. Exclusion: Ineligible setting.

279. Jabre P, Combes X, Lapostolle F, et al. Etomidate versus ketamine for rapid sequence intubation in acutely ill patients: a multicentre randomised controlled trial. Lancet. 2009 Jul 25;374(9686):293-300. doi: 10.1016/S0140-6736(09)60949-1. PMID: 19573904. Exclusion: Ineligible setting. 
280. Jabre P, Combes X, Leroux B, et al. Use of gum elastic bougie for prehospital difficult intubation. Am J Emerg Med. 2005

Jul;23(4):552-5. doi:

10.1016/j.ajem.2004.12.005. PMID: 16032630. Exclusion: Ineligible comparison or no comparison.

281. Jacoby J, Heller M, Nicholas J, et al. Etomidate versus midazolam for out-ofhospital intubation: a prospective, randomized trial. Ann Emerg Med. 2006 Jun;47(6):525-30. doi: 10.1016/j.annemergmed.2005.12.009. PMID: 16713778. Exclusion: KQ4 Triage.

282. Jain U, McCunn M, Smith CE, et al. Management of the traumatized airway. Anesthesiology. 2016 Jan;124(1):199-206. doi: 10.1097/ALN.0000000000000903. PMID: 26517857. Exclusion: Ineligible comparison or no comparison.

283. James DN, Voskresensky IV, Jack M, et al. Emergency airway management in critically injured patients: a survey of U.S. aeromedical transport programs. Resuscitation. 2009 Jun;80(6):650-7. doi: 10.1016/j.resuscitation.2009.02.020. PMID: 19375211. Exclusion: Ineligible comparison or no comparison.

284. Jamil SN, Alam M, Usmani H, et al. A study of the use of Laryngeal Mask Airway (LMA) in children and its comparison with endotracheal intubation. Indian J Anaesth. 2009 Apr;53(2):174-8. PMID: 20640119. Exclusion: Ineligible setting.

285. Jarvis JL. Advanced airway management in cardiac arrest: what does the literature say? ETI seems better than SGAs, but no advanced airway may be even better. EMS World. 2017 Jan;46(1):51-6. PMID: 29782722. Exclusion: Ineligible study design.

286. Jarvis JL, Barton D, Wang H. Defining the plateau point: when are further attempts futile in out-of-hospital advanced airway management? Resuscitation. 2018a Sep;130:57-60. doi: 10.1016/j.resuscitation.2018.07.002. PMID: 29983393. Exclusion: Ineligible comparison or no comparison.

287. Jarvis JL, Gonzales J, Johns D, et al. Implementation of a clinical bundle to reduce out-of-hospital peri-intubation hypoxia. Ann Emerg Med. 2018b Sep;72(3):272-9.e1. doi: 10.1016/j.annemergmed.2018.01.044. PMID: 29530653. Exclusion: Ineligible comparison or no comparison.
288. Javaudin F, Desce N, Le Bastard Q, et al. Impact of pre-hospital vital parameters on the neurological outcome of out-of-hospital cardiac arrest: results from the French National Cardiac Arrest Registry. Resuscitation. 2018 Dec;133:5-11. doi: 10.1016/j.resuscitation.2018.09.016. PMID: 30253228. Exclusion: Ineligible intervention.

289. Jemmett ME, Kendal KM, Fourre MW, et al. Unrecognized misplacement of endotracheal tubes in a mixed urban to rural emergency medical services setting. Acad Emerg Med. 2003 Sep;10(9):961-5. doi: 10.1111/j.1553-2712.2003.tb00652.x. PMID: 12957980. Exclusion: Ineligible comparison or no comparison.

290. Jennings PA, Cameron P, Walker T, et al. Out-of-hospital cardiac arrest in Victoria: rural and urban outcomes. Med J Aust. 2006 Aug 07;185(3):135-9. PMID: 16893352. Exclusion: Ineligible comparison or no comparison.

291. Jeong S, Ahn KO, Shin SD. The role of prehospital advanced airway management on outcomes for out-of-hospital cardiac arrest patients: a meta-analysis. Am J Emerg Med. 2016 Nov;34(11):2101-6. doi: 10.1016/j.ajem.2016.07.025. PMID: 27503061. Exclusion: Studies that meet other criteria but setting is in Emergency department.

292. Jiang J, Ma D, Li B, et al. Video laryngoscopy does not improve the intubation outcomes in emergency and critical patients - a systematic review and meta-analysis of randomized controlled trials. Crit Care. 2017 Nov 24;21(1):288. doi: 10.1186/s13054-017-1885-9. PMID: 29178953. Exclusion: Systematic review used to identify primary studies.

293. Johannigman JA, Branson RD, Johnson DJ, et al. Out-of-hospital ventilation: bag--valve device vs transport ventilator. Acad Emerg Med. 1995 Aug;2(8):719-24. doi: 10.1111/j.1553-2712.1995.tb03624.x. PMID: 7584751. Exclusion: Ineligible comparison or no comparison.

294. Jones JH, Murphy MP, Dickson RL, et al. Emergency physician-verified out-ofhospital intubation: miss rates by paramedics. Acad Emerg Med. 2004 Jun;11(6):707-9. doi: 10.1197/j.aem.2003.12.026. PMID: 15175215. Exclusion: Ineligible comparison or no comparison. 
295. Jurkovich GJ, Rivara FP, Gurney JG, et al. Effects of alcohol intoxication on the initial assessment of trauma patients. Ann Emerg Med. 1992 Jun;21(6):704-8. doi: 10.1016/s0196-0644(05)82783-0. PMID: 1590611. Exclusion: Ineligible setting.

296. Kamiutsuri K, Okutani R, Kozawa S. Analysis of prehospital endotracheal intubation performed by emergency physicians: retrospective survey of a single emergency medical center in Japan. J Anesth. 2013 Jun;27(3):374-9. doi: 10.1007/s00540-012-1528-x. PMID: 23238811. Exclusion: Ineligible comparison or no comparison.

297. Karamanos E, Talving P, Skiada D, et al. Is prehospital endotracheal intubation associated with improved outcomes in isolated severe head injury? A matched cohort analysis. Prehospital Disaster Med. 2014 Feb;29(1):32-6. doi: 10.1017/S1049023X13008947. PMID: 24330753. Exclusion: Ineligible comparison or no comparison.

298. Karch SB, Lewis T, Young S, et al. Field intubation of trauma patients: complications, indications, and outcomes. Am J Emerg Med. 1996 Nov;14(7):617-9. doi: 10.1016/S0735-6757(96)90073-X. PMID: 8906755. Exclusion: Ineligible comparison or no comparison.

299. Karisik M, Janjevic D, Sorbello M. Fiberoptic bronchoscopy versus video laryngoscopy in pediatric airway management. Acta Clin Croat. 2016 Mar;55 Suppl 1:51-4. PMID: 27276772. Exclusion: Ineligible study design.

300. Katz SH, Falk JL. Misplaced endotracheal tubes by paramedics in an urban emergency medical services system. Ann Emerg Med. 2001 Jan;37(1):32-7. doi: 10.1067/mem.2001.112098. PMID: 11145768. Exclusion: Ineligible comparison or no comparison.

301. Kempema J, Trust MD, Ali S, et al. Prehospital endotracheal intubation vs extraglottic airway device in blunt trauma. Am J Emerg Med. 2015 Aug;33(8):1080-3. doi: 10.1016/j.ajem.2015.04.046. PMID: 25963681. Exclusion: Unreconciled Publication Errors.
302. Kenny JF, Molloy K, Pollack M, et al. Nebulized lidocaine as an adjunct to endotracheal intubation in the prehospital setting. Prehospital Disaster Med. 1996 OctDec;11(4):312-3. doi: 10.1017/s1049023x00043193. PMID: 10163615. Exclusion: Ineligible comparison or no comparison.

303. Kerrey BT, Mittiga MR, Rinderknecht AS, et al. Reducing the incidence of oxyhaemoglobin desaturation during rapid sequence intubation in a paediatric emergency department. BMJ Qual Saf. 2015 Nov;24(11):709-17. doi: 10.1136/bmjqs2014-003713. PMID: 26183713. Exclusion: Studies that meet other criteria but setting is in Emergency department.

304. Kerrey BT, Rinderknecht AS, Geis GL, et al. Rapid sequence intubation for pediatric emergency patients: higher frequency of failed attempts and adverse effects found by video review. Ann Emerg Med. 2012 Sep;60(3):251-9. doi: 10.1016/j.annemergmed.2012.02.013. PMID: 22424653. Exclusion: Studies that meet other criteria but setting is in Emergency department.

305. Kerslake D, Oglesby AJ, Di Rollo N, et al. Tracheal intubation in an urban emergency department in Scotland: a prospective, observational study of 3738 intubations. Resuscitation. 2015 Apr;89:20-4. doi: 10.1016/j.resuscitation.2015.01.005. PMID: 25613360. Exclusion: Studies that meet other criteria but setting is in Emergency department.

306. Kesterson J, Silvestri S, Papa L, et al. Comparison of survival to hospital discharge in out-of-hospital trauma patients managed with an endotracheal tube compared to bagvalve-mask...2007 Society for Academic Emergency Medicine Annual Meeting. Acad Emerg Med. 2007;14:S111-S. Exclusion: Ineligible study design.

307. Khosravan S, Alami A, Hamzei A, et al. Comparing the effectiveness of airway management devices in pre-hospital emergency care: a randomized clinical trial. Pak J Med Sci. 2015 Jul-Aug;31(4):946-9. doi: 10.12669/pjms.314.7296. PMID: 26430435. Exclusion: Ineligible comparison or no comparison. 
308. Klemen P, Grmec S. Effect of pre-hospital advanced life support with rapid sequence intubation on outcome of severe traumatic brain injury. Acta Anaesthesiol Scand. 2006 Nov;50(10):1250-4. doi: 10.1111/j.13996576.2006.01039.x. PMID: 17067325. Exclusion: Ineligible comparison or no comparison.

309. Klingberg C, Kornhall D, Gryth D, et al. Checklists in pre-hospital advanced airway management. Acta Anaesthesiol Scand. 2019 Aug 22;22:22. doi: 10.1111/aas.13460. PMID: 31436306. Exclusion: Ineligible intervention.

310. Ko BS, Ahn R, Ryoo SM, et al. Prevalence and outcomes of endotracheal intubationrelated cardiac arrest in the ED. Am J Emerg Med. 2015 Nov;33(11):1642-5. doi: 10.1016/j.ajem.2015.07.083. PMID: 26324004. Exclusion: Ineligible comparison or no comparison.

311. Kociszewski C, Thomas SH, Harrison T, et al. Etomidate versus succinylcholine for intubation in an air medical setting. Am J Emerg Med. 2000 Nov;18(7):757-63. doi: 10.1053/ajem.2000.18033. PMID: 11103724. Exclusion: KQ4 Triage.

312. Koefoed-Nielsen J, Christensen EF, Melchiorsen H, et al. Acute myocardial infarction: does pre-hospital treatment increase survival? Eur J Emerg Med. 2002 Sep;9(3):210-6. doi: 10.1097/00063110200209000-00002. PMID: 12394616. Exclusion: Ineligible intervention.

313. Kramer-Johansen J, Wik L, Steen PA. Advanced cardiac life support before and after tracheal intubation--direct measurements of quality. Resuscitation. 2006 Jan;68(1):61-9. doi: 10.1016/j.resuscitation.2005.05.020. PMID: 16325329. Exclusion: Ineligible comparison or no comparison.

314. Krisanda TJ, Eitel DR, Hess D, et al. An analysis of invasive airway management in a suburban emergency medical services system. Prehospital Disaster Med. 1992 Apr-Jun;7(2):121-6. doi: 10.1017/s1049023x00039340. PMID: 10149688. Exclusion: Ineligible intervention.

315. Krmpotic K, Writer H. Cardiorespiratory arrest in children (out of hospital). Clin Evid (Online). 2015 Dec 18;18:18. PMID: 26689353. Exclusion: Systematic review used to identify primary studies.
316. Kulla M, Helm M, Lefering R, et al. Prehospital endotracheal intubation and chest tubing does not prolong the overall resuscitation time of severely injured patients: a retrospective, multicentre study of the Trauma Registry of the German Society of Trauma Surgery. Emerg Med J. 2012 Jun;29(6):497-501. doi: 10.1136/emj.2010.107391. PMID: 21795295. Exclusion: Ineligible comparison or no comparison.

317. Kupas DF, Kauffman KF, Wang HE. Effect of airway-securing method on prehospital endotracheal tube dislodgment. Prehosp Emerg Care. 2010 Jan-Mar;14(1):26-30. doi: 10.3109/10903120903144932. PMID: 19947864. Exclusion: Ineligible comparison or no comparison.

318. Kurz MC, Prince DK, Christenson J, et al. Association of advanced airway device with chest compression fraction during out-ofhospital cardiopulmonary arrest.

Resuscitation. 2016 Jan;98:35-40. doi: 10.1016/j.resuscitation.2015.10.011. PMID: 26520783. Exclusion: Ineligible outcome.

319. Langhan ML, Emerson BL, Nett S, et al. End-tidal carbon dioxide use for tracheal intubation: analysis from the National Emergency Airway Registry for Children (NEAR4KIDS) Registry. Pediatr Crit Care Med. 2018 Feb;19(2):98-105. doi: 10.1097/PCC.0000000000001372. PMID: 29140968. Exclusion: Ineligible setting.

320. Lansom JD, Curtis K, Goldsmith H, et al. The effect of prehospital intubation on treatment times in patients with suspected traumatic brain injury. Air Med J. 2016 SepOct;35(5):295-300. doi: 10.1016/j.amj.2016.04.019. PMID: 27637440. Exclusion: Ineligible comparison or no comparison.

321. Lavonas EJ, Ohshimo S, Nation K, et al. Advanced airway interventions for paediatric cardiac arrest: a systematic review and meta-analysis. Resuscitation. 2019 May;138:114-28. doi: 10.1016/j.resuscitation.2019.02.040. PMID: 30862528. Exclusion: Systematic review used to identify primary studies.

322. Le Cong M. Flying doctor emergency airway registry: a 3-year, prospective, observational study of endotracheal intubation by the Queensland section of the Royal Flying Doctor Service of Australia. Emerg Med J. 2012 Mar;29(3):249-50. doi: 10.1136/emj.2010.100651. PMID: 20844099. Exclusion: Ineligible comparison or no comparison. 
323. Lecky F, Bryden D, Little R, et al. Emergency intubation for acutely ill and injured patients. Cochrane Database Syst Rev. 2008 Apr 16(2):CD001429. doi: 10.1002/14651858.CD001429.pub2. PMID: 18425873. Exclusion: Systematic review used to identify primary studies.

324. Lee JJ, Kim JA, Gwak MS, et al. Evaluation of the Cobra perilaryngeal airway (CPLA) as an airway conduit. Eur J Anaesthesiol. 2007 Oct;24(10):852-5. doi: 10.1017/S0265021507000877. PMID: 17582247. Exclusion: Ineligible setting.

325. Lefrancois DP, Dufour DG. Use of the esophageal tracheal combitube by basic emergency medical technicians. Resuscitation. 2002 Jan;52(1):77-83. doi: 10.1016/s0300-9572(01)00441-5. PMID: 11801352. Exclusion: Ineligible comparison or no comparison.

326. Lemaitre EL, Tritsch L, Noll E, et al. Effectiveness of intubating laryngeal mask airway in managing out-of-hospital cardiac arrest by non-physicians. Resuscitation. 2019 Mar;136:61-9. doi: 10.1016/j.resuscitation.2018.12.004. PMID: 30572066. Exclusion: Ineligible comparison or no comparison.

327. Lenartova L, Janciak I, Wilbacher I, et al. Severe traumatic brain injury in Austria III: prehospital status and treatment. Wien Klin Wochenschr. 2007 Feb;119(1-2):35-45. doi: 10.1007/s00508-006-0762-3. PMID:

17318749. Exclusion: Ineligible comparison or no comparison.

328. Leung SK, Cruz AT, Macias CG, et al. Improving pediatric emergency care by implementing an eligible learner endotracheal intubation policy. Pediatr Emerg Care. 2016 Apr;32(4):205-9. doi: 10.1097/PEC.0000000000000764. PMID: 26990848. Exclusion: Studies that meet other criteria but setting is in Emergency department.

329. Lewis CT, Brown J, Inglis AC, et al. Emergency intubation in trauma in KwaZulu-Natal Province, South Africa. Samj, S. 2018 Jul 25;108(8):660-6. doi: 10.7196/SAMJ.2018.v108i8.12670. PMID: 30182882. Exclusion: Ineligible study design.

330. Li J, Murphy-Lavoie H, Bugas C, et al. Complications of emergency intubation with and without paralysis. Am J Emerg Med. 1999 Mar;17(2):141-3. doi: 10.1016/s07356757(99)90046-3. PMID: 10102312.

Exclusion: Studies that meet other criteria but setting is in Emergency department.
331. Lin CC, Yu JH, Lin CC, et al. Postintubation hemodynamic effects of intravenous lidocaine in severe traumatic brain injury. Am J Emerg Med. 2012 Nov;30(9):1782-7. doi: 10.1016/j.ajem.2012.02.013. PMID: 22633717. Exclusion: Ineligible setting.

332. Lin YR, Wu HP, Huang CY, et al. Significant factors in predicting sustained ROSC in paediatric patients with traumatic out-of-hospital cardiac arrest admitted to the emergency department. Resuscitation. 2007 Jul;74(1):83-9. doi:

10.1016/j.resuscitation.2006.11.022. PMID: 17353084. Exclusion: Ineligible intervention.

333. Lioutas VA, Marchina S, Caplan LR, et al. Endotracheal intubation and in-hospital mortality after intracerebral hemorrhage. Cerebrovasc Dis. 2018;45(5-6):270-8. doi: 10.1159/000489273. PMID: 29898436. Exclusion: Ineligible setting.

334. Littlewood K, Durbin CG, Jr. Evidencedbased airway management. Respir Care. 2001 Dec;46(12):1392-405; discussion 4067. PMID: 11728299. Exclusion: Systematic review used to identify primary studies.

335. Liu EH, Goy RW, Lim Y, et al. Success of tracheal intubation with intubating laryngeal mask airways: a randomized trial of the LMA Fastrach and LMA CTrach. Anesthesiology. 2008 Apr;108(4):621-6. doi: 10.1097/ALN.0b013e318167af61. PMID: 18362593. Exclusion: Ineligible setting.

336. Lockey D, Davies G, Coats T. Survival of trauma patients who have prehospital tracheal intubation without anaesthesia or muscle relaxants: observational study. Bmj. 2001 Jul 21;323(7305):141. doi: 10.1136/bmj.323.7305.141. PMID: 11463683. Exclusion: Ineligible comparison or no comparison.

337. Lockey DJ, Avery P, Harris T, et al. A prospective study of physician pre-hospital anaesthesia in trauma patients: oesophageal intubation, gross airway contamination and the 'quick look' airway assessment. BMC anesthesiol. 2013 Sep 11;13(1):21. doi: 10.1186/1471-2253-13-21. PMID: 24024531. Exclusion: Ineligible comparison or no comparison. 
338. Lockey DJ, Healey B, Crewdson K, et al. Advanced airway management is necessary in prehospital trauma patients. Br J Anaesth. 2015 Apr;114(4):657-62. doi:

10.1093/bja/aeu412. PMID: 25540067.

Exclusion: Ineligible comparison or no comparison.

339. Loiselle JM, Cone DC. Effect of out-ofhospital pediatric endotracheal intubation on survival and neurological outcome: a controlled trial. Ann Emerg Med. 2001 Sep;38(3):352-3. PMID: 11548734. Exclusion: Ineligible study design.

340. Long E, Sabato S, Babl FE. Endotracheal intubation in the pediatric emergency department. Paediatr Anaesth. 2014 Dec;24(12):1204-11. doi: 10.1111/pan.12490. PMID: 25039321. Exclusion: Studies that meet other criteria but setting is in Emergency department.

341. Lossius HM, Røislien J, Lockey DJ. Patient safety in pre-hospital emergency tracheal intubation: a comprehensive meta-analysis of the intubation success rates of EMS providers. Crit Care. 2012 Feb 11;16(1):R24. doi: 10.1186/cc11189. PMID: 22325973. Exclusion: Systematic review used to identify primary studies.

342. Loughnan A, Deng C, Dominick F, et al. A single-centre, randomised controlled feasibility pilot trial comparing performance of direct laryngoscopy versus videolaryngoscopy for endotracheal intubation in surgical patients. Pilot feasibility stud. 2019;5:50. doi: 10.1186/s40814-019-0433-6. PMID: 30976455. Exclusion: Ineligible setting.

343. Lowe L, Sagehorn K, Madsen R. The effect of a rapid sequence induction protocol on intubation success rate in an air medical program. Air Med J. 1998 JulSep;17(3):101-4. doi: 10.1016/s1067991x(98)90103-1. PMID: 10181919. Exclusion: Ineligible comparison or no comparison.

344. Lupton JR, Schmicker R, Daya MR, et al. Effect of initial airway strategy on time to epinephrine administration in patients with out-of-hospital cardiac arrest. Resuscitation. 2019 Jun;139:314-20. doi: 10.1016/j.resuscitation.2019.03.015. PMID: 30902690. Exclusion: No new data.

345. Lyon RM, Ferris JD, Young DM, et al. Field intubation of cardiac arrest patients: a dying art? Emerg Med J. 2010 Apr;27(4):321-3. doi: 10.1136/emj.2009.076737. PMID: 20385694. Exclusion: Ineligible comparison or no comparison.
346. Lyon RM, Perkins ZB, Chatterjee D, et al. Significant modification of traditional rapid sequence induction improves safety and effectiveness of pre-hospital trauma anaesthesia. Crit Care. 2015 Apr 01;19:134. doi: 10.1186/s13054-015-0872-2. PMID: 25879683. Exclusion: KQ4 Triage.

347. Ma OJ, Atchley RB, Hatley T, et al. Intubation success rates improve for an air medical program after implementing the use of neuromuscular blocking agents. Am J Emerg Med. 1998 Mar;16(2):125-7. doi: 10.1016/s0735-6757(98)90027-4. PMID 9517684. Exclusion: Ineligible outcome.

348. Mackay CA, Terris J, Coats TJ. Prehospital rapid sequence induction by emergency physicians: is it safe? Emerg Med J. 2001 Jan;18(1):20-4. doi: 10.1136/emj.18.1.20. PMID: 11310456. Exclusion: KQ4 Triage.

349. MacNab AJ, MacPhail I, MacNab MK, et al. A comparison of intubation success for paediatric transport team paramedics using lighted vs regular tracheal tube stylets. Paediatr Anaesth. 1998;8(3):215-20. doi: 10.1046/j.1460-9592.1998.00742.x. PMID: 9608966. Exclusion: Ineligible population.

350. Maertens VL, De Smedt LE, Lemoyne S, et al. Patients with cardiac arrest are ventilated two times faster than guidelines recommend: an observational prehospital study using tracheal pressure measurement. Resuscitation. 2013 Jul;84(7):921-6. doi: 10.1016/j.resuscitation.2012.11.015. PMID: 23178868. Exclusion: Ineligible outcome.

351. Maeyama H, Naito H, Guyette FX, et al. Intubation during a medevac flight: safety and effect on total prehospital time in the helicopter emergency medical service system. Scand J Trauma Resusc Emerg Med. 2020 Sep 07;28(1):89. doi: 10.1186/s13049-020-00784-z. PMID: 32894186. Exclusion: KQ4 Triage.

352. Mandavia DP, Qualls S, Rokos I. Emergency airway management in penetrating neck injury. Ann Emerg Med. 2000 Mar;35(3):221-5. doi: 10.1016/s01960644(00)70071-0. PMID: 10692187. Exclusion: Ineligible setting.

353. Martin SE, Ochsner MG, Jarman RH, et al. Use of the laryngeal mask airway in air transport when intubation fails. J Trauma. 1999 Aug;47(2):352-7. PMID: 10452473. Exclusion: Ineligible comparison or no comparison. 
354. Martin-Gill C, Prunty HA, Ritter SC, et al. Risk factors for unsuccessful prehospital laryngeal tube placement. Resuscitation. 2015 Jan;86:25-30. doi:

10.1016/j.resuscitation.2014.10.015. PMID: 25447434. Exclusion: KQ4 Triage.

355. Martinon C, Duracher C, Blanot S, et al. Emergency tracheal intubation of severely head-injured children: changing daily practice after implementation of national guidelines. Pediatr Crit Care Med. 2011 Jan;12(1):65-70. doi: 10.1097/PCC.0b013e3181e2a244. PMID: 20473241. Exclusion: Ineligible intervention.

356. McBrien ME, Pollok AJ, Steedman DJ. Advanced airway control in trauma resuscitation. Arch Emerg Med. 1992 Jun;9(2):177-80. doi: 10.1136/emj.9.2.177. PMID: 1388492. Exclusion: Ineligible comparison or no comparison.

357. McIntosh SE, Swanson ER, McKeone A, et al. Location of airway management in air medical transport. Prehosp Emerg Care. 2008 Oct-Dec;12(4):438-42. doi: 10.1080/10903120802301518. PMID: 18924006. Exclusion: Ineligible setting.

358. McQueen C, Crombie N, Hulme J, et al. Prehospital anaesthesia performed by physician/critical care paramedic teams in a major trauma network in the UK: a 12 month review of practice. Emerg Med J. 2015 Jan;32(1):65-9. doi: 10.1136/emermed-2013-202890. PMID: 24132327. Exclusion: KQ4 Triage.

359. Mendez DR, Goto CS, Abramo TJ, et al. Safety and efficacy of rocuronium for controlled intubation with paralytics in the pediatric emergency department. Pediatr Emerg Care. 2001 Aug;17(4):233-6. doi: 10.1097/00006565-200108000-00001. PMID: 11493818. Exclusion: Studies that meet other criteria but setting is in Emergency department.

360. Meyer G, Orliaguet G, Blanot S, et al. Complications of emergency tracheal intubation in severely head-injured children. Paediatr Anaesth. 2000;10(3):253-60. doi: 10.1046/j.1460-9592.2000.00496.x. PMID: 10792740. Exclusion: Ineligible comparison or no comparison.

361. Michetti CP, Maguire JF, Kaushik A, et al. Single-drug sedation with fentanyl for prehospital postintubation sedation in trauma patients. J Trauma Acute Care Surg. 2012 Apr;72(4):924-9. doi:

10.1097/TA.0b013e3182479884. PMID: 22491606. Exclusion: KQ4 Triage.
362. Miklus RM, Elliott C, Snow N. Surgical cricothyrotomy in the field: experience of a helicopter transport team. J Trauma. 1989 Apr;29(4):506-8. PMID: 2709460. Exclusion: Ineligible intervention.

363. Miller KA, Kimia A, Monuteaux MC, et al. Factors associated with misplaced endotracheal tubes during intubation in pediatric patients. J Emerg Med. 2016 Jul;51(1):9-18. doi: 10.1016/j.jemermed.2016.04.007. PMID: 27236246. Exclusion: Studies that meet other criteria but setting is in Emergency department.

364. Mort TC. Extubating the difficult airway: formulating the management strategy: use of accessory airway devices and alternative techniques may be key. J Crit Ill. 2003;18(5):210-7. Exclusion: Ineligible publication type.

365. Mort TC. Emergency tracheal intubation: complications associated with repeated laryngoscopic attempts. Anesth Analg. 2004 Aug;99(2):607-13, table of contents. doi: 10.1213/01.Ane.0000122825.04923.15. PMID: 15271750. Exclusion: Ineligible setting.

366. Muller JU, Semmel T, Stepan R, et al. The use of the laryngeal tube disposable by paramedics during out-of-hospital cardiac arrest: a prospectively observational study (2008-2012). Emerg Med J. 2013 Dec;30(12):1012-6. doi: 10.1136/emermed2012-201923. PMID: 23307754. Exclusion: Ineligible comparison or no comparison.

367. Murphy DL, Rea TD, McCoy AM, et al. Inclined position is associated with improved first pass success and laryngoscopic view in prehospital endotracheal intubations. Am J Emerg Med. 2019 May;37(5):937-41. doi: 10.1016/j.ajem.2019.02.038. PMID: 30826211. Exclusion: KQ4 Triage.

368. Murphy-Macabobby M, Marshall WJ, Schneider C, et al. Neuromuscular blockade in aeromedical airway management. Ann Emerg Med. 1992 Jun;21(6):664-8. doi: 10.1016/s0196-0644(05)82776-3. PMID: 1590604. Exclusion: Ineligible comparison or no comparison. 
369. Naito H, Yumoto T, Yorifuji T, et al. Improved outcomes for out-of-hospital cardiac arrest patients treated by emergency life-saving technicians compared with basic emergency medical technicians: a JCS-ReSS study report. Resuscitation. 2020

Aug; 153:251-7. doi:

10.1016/j.resuscitation.2020.05.007. PMID: 32422240. Exclusion: Ineligible comparison or no comparison.

370. Nakao S, Kimura A, Hagiwara Y, et al. Trauma airway management in emergency departments: a multicentre, prospective, observational study in Japan. BMJ Open. 2015 Feb 04;5(2):e006623. doi: 10.1136/bmjopen-2014-006623. PMID: 25652800. Exclusion: Studies that meet other criteria but setting is in Emergency department.

371. Nakayama DK, Gardner MJ, Rowe MI. Emergency endotracheal intubation in pediatric trauma. Ann Surg. 1990 Feb;211(2):218-23. doi: 10.1097/00000658199002000-00015. PMID: 2301999.

Exclusion: Ineligible comparison or no comparison.

372. Nakstad AR, Heimdal HJ, Strand T, et al Incidence of desaturation during prehospital rapid sequence intubation in a physicianbased helicopter emergency service. Am J Emerg Med. 2011 Jul;29(6):639-44. doi: 10.1016/j.ajem.2010.01.013. PMID: 20825848. Exclusion: Ineligible comparison or no comparison.

373. Naumann DN, McLaughlin A, Midwinter $\mathrm{MJ}$, et al. Prehospital rapid sequence induction following trauma in the era of regional networks for major trauma. Eur J Emerg Med. 2017 Aug;24(4):243-8. doi: 10.1097/MEJ.0000000000000364. PMID: 26716997. Exclusion: Ineligible comparison or no comparison.

374. Nevin DG, Green SJ, Weaver AE, et al. An observational study of paediatric prehospital intubation and anaesthesia in 1933 children attended by a physician-led, prehospital trauma service. Resuscitation. 2014 Feb;85(2):189-95. doi:

10.1016/j.resuscitation.2013.10.006. PMID: 24145041. Exclusion: Ineligible comparison or no comparison.
375. Newgard CD, Koprowicz K, Wang H, et al. Variation in the type, rate, and selection of patients for out-of-hospital airway procedures among injured children and adults. Acad Emerg Med. 2009 Dec;16(12):1269-76. doi: 10.1111/j.15532712.2009.00604.x. PMID: 20053248. Exclusion: Ineligible comparison or no comparison.

376. Newton A, Ratchford A, Khan I. Incidence of adverse events during prehospital rapid sequence intubation: a review of one year on the London Helicopter Emergency Medical Service. J Trauma. 2008 Feb;64(2):487-92. doi: 10.1097/TA.0b013e31802e7476. PMID: 18301219. Exclusion: Ineligible comparison or no comparison.

377. Niemann JT, Stratton SJ, Cruz B, et al. Endotracheal drug administration during out-of-hospital resuscitation: where are the survivors? Resuscitation. 2002 May;53(2):153-7. doi: 10.1016/s03009572(02)00004-7. PMID: 12009218. Exclusion: Ineligible intervention.

378. Ochs M, Davis D, Hoyt D, et al. Paramedicperformed rapid sequence intubation of patients with severe head injuries. Ann Emerg Med. 2002 Aug;40(2):159-67. doi: 10.1067/mem.2002.126397. PMID: 12140494. Exclusion: Ineligible comparison or no comparison.

379. Ochs M, Vilke GM, Chan TC, et al. Successful prehospital airway management by EMT-Ds using the combitube. Prehosp Emerg Care. 2000 Oct-Dec;4(4):333-7. doi: 10.1080/10903120090941065. PMID: 11045413. Exclusion: Ineligible comparison or no comparison.

380. O'Connor R E, Megargel RE, Schnyder ME, et al. Paramedic success rate for blind nasotracheal intubation is improved with the use of an endotracheal tube with directional tip control. Ann Emerg Med. 2000 Oct;36(4):328-32. doi: 10.1067/mem.2000.108316. PMID: 11020679. Exclusion: Ineligible intervention.

381. Oglesby AJ, Graham CA, Beard D, et al. Paediatric intubation in Scottish emergency departments. Paediatr Anaesth. 2003 Sep;13(7):589-95. doi: 10.1046/j.14609592.2003.01107.x. PMID: 12950859. Exclusion: Studies that meet other criteria but setting is in Emergency department. 
382. Ohashi-Fukuda N, Fukuda T, Doi K, et al. Effect of prehospital advanced airway management for pediatric out-of-hospital cardiac arrest. Resuscitation. 2017a May;114:66-72. doi: 10.1016/j.resuscitation.2017.03.002. PMID: 28267617. Exclusion: KQ4 Triage.

383. Ohta R, Shimabukuro A. Rural physicians' scope of practice on remote islands: a case report of severe pneumonia that required overnight artificial airway management. J Rural Med. 2017 May;12(1):53-5. doi: 10.2185/jrm.2925. PMID: 28593019. Exclusion: Ineligible comparison or no comparison.

384. Okubo M, Komukai S, Izawa J, et al. Prehospital advanced airway management for paediatric patients with out-of-hospital cardiac arrest: a nationwide cohort study. Resuscitation. 2019 Sep 17;17:17. doi: 10.1016/j.resuscitation.2019.09.007. PMID: 31539609. Exclusion: Ineligible comparison or no comparison.

385. Olsen J, Lenz T. Direct vs. video laryngoscopy in a Helicopter Emergency Medical Service (HEMS) setting: a retrospective comparison. Air Med J. 2018;37(5):292-3. Exclusion: Ineligible publication type.

386. Omert L, Yeaney W, Mizikowski S, et al. Role of the emergency medicine physician in airway management of the trauma patient. J Trauma. 2001 Dec;51(6):1065-8. doi: 10.1097/00005373-200112000-00007. PMID: 11740252. Exclusion: Studies that meet other criteria but setting is in Emergency department.

387. Ono Y, Kakamu T, Kikuchi H, et al. Expertperformed endotracheal intubation-related complications in trauma patients: incidence, possible risk factors, and outcomes in the prehospital setting and emergency department. Emerg Med Int. 2018 Jun;2018:5649476. doi: 10.1155/2018/5649476. PMID: 29984001. Exclusion: Studies that meet other criteria but setting is in Emergency department.

388. Ono Y, Kikuchi H, Hashimoto K, et al. Emergency endotracheal intubation-related adverse events in bronchial asthma exacerbation: can anesthesiologists attenuate the risk? J. 2015 Oct;29(5):678-85. doi: https://dx.doi.org/10.1007/s00540-0152003-2. PMID: 25801541. Exclusion: Studies that meet other criteria but setting is in Emergency department.
389. Oswalt JL, Hedges JR, Soifer BE, et al. Analysis of trauma intubations. Am J Emerg Med. 1992 Nov;10(6):511-4. doi: 10.1016/0735-6757(92)90173-u. PMID: 1388374. Exclusion: Ineligible comparison or no comparison.

390. Ozkurtul O, Struck MF, Fakler J, et al. Physician-based on-scene airway management in severely injured patients and in-hospital consequences: is the misplaced intubation an underestimated danger in trauma management? Trauma surg. 2019;4(1):e000271. doi: 10.1136/tsaco2018-000271. PMID: 30899797. Exclusion: Ineligible comparison or no comparison.

391. Pace SA, Fuller FP. Out-of-hospital succinylcholine-assisted endotracheal intubation by paramedics. Ann Emerg Med. 2000 Jun;35(6):568-72. PMID: 10828769. Exclusion: Ineligible study design.

392. Page D, Boyer C. Studies underway to evaluate the use of supraglottic airways vs. intubation in cardiac arrest patients. JEMS. 2017;42(1):50-1. Exclusion: Ineligible publication type.

393. Pakkanen T, Kamarainen A, Huhtala H, et al. Physician-staffed helicopter emergency medical service has a beneficial impact on the incidence of prehospital hypoxia and secured airways on patients with severe traumatic brain injury. Scand J Trauma Resusc Emerg Med. 2017 Sep 15;25(1):94. doi: 10.1186/s13049-017-0438-1. PMID: 28915898. Exclusion: Ineligible outcome.

394. Pakkanen T, Virkkunen I, Kamarainen A, et al. Pre-hospital severe traumatic brain injury - comparison of outcome in paramedic versus physician staffed emergency medical services. Scand J Trauma Resusc Emerg Med. 2016 Apr 29;24:62. doi: 10.1186/s13049-016-0256-x. PMID: 27130216. Exclusion: Ineligible comparison or no comparison.

395. Pakkanen T, Virkkunen I, Silfvast T, et al. One-year outcome after prehospital intubation. Acta Anaesthesiol Scand. 2015 Apr;59(4):524-30. doi: 10.1111/aas.12483. PMID: 25790242. Exclusion: Ineligible comparison or no comparison.

396. Palmon SC, Liu M, Moore LE, et al. Capnography facilitates tight control of ventilation during transport. Crit Care Med. 1996 Apr;24(4):608-11. doi: 10.1097/00003246-199604000-00010. PMID: 8612411. Exclusion: Ineligible intervention. 
397. Panchal AR, Finnegan G, Way DP, et al. Assessment of paramedic performance on difficult airway simulation. Prehosp Emerg Care. 2020 Nov 21:1-10. doi: 10.3109/10903127.2015.1102993. PMID: 27870588. Exclusion: Ineligible population.

398. Park JH, Lee JS, Nam SB, et al. Standard versus rotation technique for insertion of supraglottic airway devices: systematic review and meta-analysis. Yonsei Med J. 2016 Jul;57(4):987-97. doi: 10.3349/ymj.2016.57.4.987. PMID: 27189296. Exclusion: Ineligible setting.

399. Park MJ, Kwon WY, Kim K, et al. Prehospital supraglottic airway was associated with good neurologic outcome in cardiac arrest victims especially those who received prolonged cardiopulmonary resuscitation. Acad Emerg Med. 2017 Dec;24(12):1464-73. doi: 10.1111/acem.13309. PMID: 28898484. Exclusion: Ineligible outcome.

400. Parr M. Prehospital airway management for severe brain injury. Resuscitation. 2008 Mar;76(3):321-2. doi:

10.1016/j.resuscitation.2008.01.001. PMID: 18262081. Exclusion: Ineligible publication type.

401. Patrick CB, Crocker K, Fioretti J, et al. REDUCING LUNG INJURY: protective mechanical ventilation strategies in the prehospital setting. JEMS: Journal of Emergency Medical Services. 2018;43(4):40-2. Exclusion: Ineligible intervention.

402. Paul SP, Heaton P. Pre-mature babies born in the pre-hospital setting: a challenging situation. Journal of Paramedic Practice. 2013;5(2):108-12. Exclusion: Ineligible study design.

403. Paul TR, Marias M, Pons PT, et al. Adult versus pediatric prehospital trauma care: is there a difference? J Trauma. 1999 Sep;47(3):455-9. doi: 10.1097/00005373199909000-00004. PMID: 10498297. Exclusion: KQ4 Triage.

404. Pearson S. Comparison of intubation attempts and completion times before and after the initiation of a rapid sequence intubation protocol in an air medical transport program. Air Med J. 2003 NovDec;22(6):28-33. doi: 10.1016/j.amj.2003.08.004. PMID: 14762994. Exclusion: Ineligible comparison or no comparison.
405. Pelucio M, Halligan L, Dhindsa H. Out-ofhospital experience with the syringe esophageal detector device. Acad Emerg Med. 1997 Jun;4(6):563-8. doi: 10.1111/j.1553-2712.1997.tb03579.x. PMID: 9189188. Exclusion: Ineligible intervention.

406. Pepe PE, Zachariah BS, Chandra NC. Invasive airway techniques in resuscitation. Ann Emerg Med. 1993 Feb;22(2 Pt 2):393403. doi: 10.1016/s0196-0644(05)80470-6. PMID: 8434839. Exclusion: Systematic review used to identify primary studies.

407. Perkins ZB, Gunning M, Crilly J, et al. The haemodynamic response to pre-hospital RSI in injured patients. Injury. 2013b

May;44(5):618-23. doi: 10.1016/j.injury.2012.03.019. PMID: 22483540. Exclusion: Ineligible comparison or no comparison.

408. Perkins ZB, Wittenberg MD, Nevin D, et al. The relationship between head injury severity and hemodynamic response to tracheal intubation. J Trauma Acute Care Surg. 2013a Apr;74(4):1074-80. doi: 10.1097/TA.0b013e3182827305. PMID: 23511147. Exclusion: Ineligible comparison or no comparison.

409. Perron AD, Sing RF, Branas CC, et al. Predicting survival in pediatric trauma patients receiving cardiopulmonary resuscitation in the prehospital setting. Prehosp Emerg Care. 2001 Jan-Mar;5(1):69. doi: 10.1080/10903120190940245. PMID: 11194072. Exclusion: Ineligible intervention.

410. Peters J, van Wageningen B, Hendriks I, et al. First-pass intubation success rate during rapid sequence induction of prehospital anaesthesia by physicians versus paramedics. Eur J Emerg Med. 2015 Dec;22(6):391-4. doi: 10.1097/MEJ.0000000000000161. PMID: 24841771. Exclusion: KQ4 Triage.

411. Piegeler T, Neth P, Schlaepfer M, et al. Advanced airway management in an anaesthesiologist-staffed Helicopter Emergency Medical Service (HEMS): a retrospective analysis of 1047 out-ofhospital intubations. Resuscitation. 2016 Aug;105:66-9. doi: 10.1016/j.resuscitation.2016.04.020. PMID: 27241333. Exclusion: Ineligible comparison or no comparison. 
412. Pietsch U, Knapp J, Kreuzer O, et al. Advanced airway management in hoist and longline operations in mountain HEMS considerations in austere environments: a narrative review This review is endorsed by the International Commission for Mountain Emergency Medicine (ICAR MEDCOM). Scand J Trauma Resusc Emerg Med. 2018 Apr 03;26(1):23. doi: 10.1186/s13049-0180490-5. PMID: 29615073. Exclusion:

Systematic review used to identify primary studies.

413. Pollock MJ, Brown LH, Dunn KA. The perceived importance of paramedic skills and the emphasis they receive during EMS education programs. Prehosp Emerg Care. 1997 Oct-Dec;1(4):263-8. doi: 10.1080/10903129708958821. PMID: 9709368. Exclusion: Ineligible study design.

414. Poste JC, Davis DP, Ochs M, et al. Air medical transport of severely head-injured patients undergoing paramedic rapid sequence intubation. Air Med J. 2004 JulAug;23(4):36-40. doi: 10.1016/j.amj.2004.04.006. PMID: 15224081. Exclusion: KQ4 Triage.

415. Prasad NH, Brown LH. Research review. Necessity of EtCO2 monitoring in ET intubations. JEMS. 2005;30(8):42-3. Exclusion: Ineligible publication type.

416. Pratt JC, Hirshberg AJ. Endotracheal tube placement by EMT-basics in a rural EMS system. Prehosp Emerg Care. 2005 AprJun;9(2):172-5. doi: 10.1080/10903120590924564. PMID: 16036842. Exclusion: Ineligible comparison or no comparison.

417. Price B, Arthur AO, Brunko M, et al. Hemodynamic consequences of ketamine vs etomidate for endotracheal intubation in the air medical setting. Am J Emerg Med. 2013 Jul;31(7):1124-32. doi:

10.1016/j.ajem.2013.03.041. PMID:

23702065. Exclusion: KQ4 Triage.

418. Price D, Burns B. Brain injuries. Emerg Med Serv. 1999;28(6):65-70. Exclusion: Ineligible publication type.

419. Price DD, Wilson SR, Fee ME. Sidestream end-tidal carbon dioxide monitoring during helicopter transport. Air Med J. 2007 JanFeb;26(1):55-9. doi: 10.1016/j.amj.2006.10.004. PMID: 17210495. Exclusion: Ineligible intervention.
420. Price SJ, Suttner N, Aspoas AR. Have ATLS and national transfer guidelines improved the quality of resuscitation and transfer of head-injured patients? A prospective survey from a Regional Neurosurgical Unit. Injury. 2003 Nov;34(11):834-8. doi: 10.1016/s00201383(03)00028-7. PMID: 14580816. Exclusion: Ineligible outcome.

421. Probst C, Pape HC, Hildebrand F, et al. 30 years of polytrauma care: an analysis of the change in strategies and results of 4849 cases treated at a single institution. Injury. 2009 Jan;40(1):77-83. doi: 10.1016/j.injury.2008.10.004. PMID: 19117558. Exclusion: Ineligible comparison or no comparison.

422. Prokakis C, Koletsis EN, Dedeilias P, et al. Airway trauma: a review on epidemiology, mechanisms of injury, diagnosis and treatment. J Cardiothorac Surg. 2014 Jun 30;9:117. doi: 10.1186/1749-8090-9-117. PMID: 24980209. Exclusion: Ineligible publication type.

423. Pugh HE, LeClerc S, McLennan J. A review of pre-admission advanced airway management in combat casualties, Helmand Province 2013. J R Army Med Corps. 2015 Jun;161(2):121-6. doi: 10.1136/jramc-2014000271. PMID: 25138107. Exclusion: Ineligible comparison or no comparison.

424. Rabitsch W, Nikolic A, Schellongowski P, et al. Evaluation of an end-tidal portable ETCO2 colorimetric breath indicator (COLIBRI). Am J Emerg Med. 2004 Jan;22(1):4-9. doi:

10.1016/j.ajem.2003.09.008. PMID: 14724870. Exclusion: Ineligible intervention.

425. Rainer TH, Marshall R, Cusack S. Paramedics, technicians, and survival from out of hospital cardiac arrest. J Accid Emerg Med. 1997 Sep;14(5):278-82. doi: 10.1136/emj.14.5.278. PMID: 9315925. Exclusion: Ineligible intervention.

426. Ramgopal S, Button SE, Owusu-Ansah S, et al. Success of pediatric intubations performed by a critical care transport service. Prehosp Emerg Care. 2020 SepOct;24(5):683-92. doi: 10.1080/10903127.2019.1699212. PMID: 31800336. Exclusion: Ineligible setting.

427. Ray DC, McKeown DW. Emergency tracheal intubation: more than just technical skill. Resuscitation. 2011 May;82(5):505-6. doi: 10.1016/j.resuscitation.2011.02.042. PMID: 21459505. Exclusion: Ineligible publication type. 
428. Reed DB, Snyder G, Hogue TD. Regional

EMS experience with etomidate for

facilitated intubation. Prehosp Emerg Care.

2002 Jan-Mar;6(1):50-3. doi:

10.1080/10903120290938779. PMID:

11789650. Exclusion: KQ4 Triage.

429. Reichert RJ, Gothard M, Gothard MD, et al. Intubation success in critical care transport: a multicenter study. Prehosp Emerg Care. 2018 Sep-Oct;22(5):571-7. doi: 10.1080/10903127.2017.1419324. PMID: 29465274. Exclusion: Ineligible population.

430. Reid C, Chan L, Tweeddale M. The who, where, and what of rapid sequence intubation: prospective observational study of emergency RSI outside the operating theatre. Emerg Med J. 2004 May;21(3):296301. doi: 10.1136/emj.2003.007344. PMID: 15107366. Exclusion: Ineligible comparison or no comparison.

431. Reinhart DJ, Simmons G. Comparison of placement of the laryngeal mask airway with endotracheal tube by paramedics and respiratory therapists. Ann Emerg Med. 1994 Aug;24(2):260-3. PMID: 8037393. Exclusion: Ineligible setting.

432. Reynolds JC, Grunau BE, Rittenberger JC, et al. The association between duration of resuscitation and favorable outcome after out-of-hospital cardiac arrest: implications for prolonging or terminating resuscitation. Circulation. 2016 Dec 20;134(25):2084-94. doi:

10.1161/CIRCULATIONAHA.116.023309. PMID: 27760796. Exclusion: Ineligible intervention.

433. Rhee KJ, O'Malley RJ. Neuromuscular blockade-assisted oral intubation versus nasotracheal intubation in the prehospital care of injured patients. Ann Emerg Med. 1994 Jan;23(1):37-42. doi: 10.1016/s01960644(94)70005-2. PMID: 8273956.

Exclusion: Ineligible comparison or no comparison.

434. Rhee KJ, Muntz CB, Donald PJ, et al. Does nasotracheal intubation increase complications in patients with skull base fractures? Ann Emerg Med. 1993 Jul;22(7):1145-7. doi: 10.1016/s01960644(05)80980-1. PMID: 8517565. Exclusion: Ineligible intervention.

435. Rhode MG, Vandborg MP, Bladt V, et al. Video laryngoscopy in pre-hospital critical care - a quality improvement study. Scand J Trauma Resusc Emerg Med. 2016 Jun 13;24:84. doi: 10.1186/s13049-016-0276-6. PMID: 27297563. Exclusion: Ineligible comparison or no comparison.
436. Rich JM, Thierbach A, Frass M. The Combitube, self-inflating bulb, and colorimetric carbon dioxide detector to advance airway management in the first echelon of the battlefield. Mil Med. 2006 May;171(5):389-95. doi:

10.7205/milmed.171.5.389. PMID: 16761887. Exclusion: Ineligible comparison or no comparison.

437. Richard J, Osmond MH, Nesbitt L, et al. Management and outcomes of pediatric patients transported by emergency medical services in a Canadian prehospital system. CJEM, Can. 2006 Jan;8(1):6-12. doi: 10.1017/s1481803500013312. PMID: 17175623. Exclusion: Ineligible comparison or no comparison.

438. Richmond NJ. BVM REVISITED: In the bag or out of control? JEMS. 2018;43(4):60. Exclusion: Ineligible publication type.

439. Ringbaek TJ, Terkelsen J, Lange P. Outcomes of acute exacerbations in COPD in relation to pre-hospital oxygen therapy. Eur Clin Respir J. 2015 May 11;2doi: 10.3402/ecrj.v2.27283. PMID: 26557264. Exclusion: Ineligible intervention.

440. Rittenberger JC, Guimond G, Platt TE, et al. Quality of BLS decreases with increasing resuscitation complexity. Resuscitation. 2006 Mar;68(3):365-9. doi: 10.1016/j.resuscitation.2005.07.019. PMID: 16377058. Exclusion: Ineligible population.

441. Riyapan S, Lubin J. Apneic oxygenation may not prevent severe hypoxemia during rapid sequence intubation: a retrospective helicopter emergency medical service study. Air Med J. 2016 Nov - Dec;35(6):365-8. doi: 10.1016/j.amj.2016.07.008. PMID: 27894561. Exclusion: KQ4 Triage.

442. Robinson MJ, Taylor J, Brett SJ, et al. Design and implementation of a large and complex trial in emergency medical services. Trials. 2019a;20(1)doi: 10.1186/s13063-019-3203-0. PMID: 30736841. Exclusion: Ineligible study design.

443. Rognas L, Hansen TM, Kirkegaard H, et al. Pre-hospital advanced airway management by experienced anaesthesiologists: a prospective descriptive study. Scand J Trauma Resusc Emerg Med. 2013 Jul 25;21:58. doi: 10.1186/1757-7241-21-58. PMID: 23883447. Exclusion: Ineligible comparison or no comparison. 
444. Rognas L, Hansen TM, Kirkegaard H, et al. Predicting the lack of ROSC during prehospital CPR: should an end-tidal CO2 of $1.3 \mathrm{kPa}$ be used as a cut-off value? Resuscitation. 2014 Mar;85(3):332-5. doi: 10.1016/j.resuscitation.2013.12.009. PMID: 24361671. Exclusion: Ineligible comparison or no comparison.

445. Romanowski KS, Palmieri TL, Sen S, et al. More than one third of intubations in patients transferred to burn centers are unnecessary: proposed guidelines for appropriate intubation of the burn patient. J Burn Care Res. 2016 Sep-Oct;37(5):e40914. doi: 10.1097/BCR.0000000000000288. PMID: 26284640. Exclusion: Ineligible setting.

446. Rose L, Gray S, Burns K, et al. Emergency department length of stay for patients requiring mechanical ventilation: a prospective observational study. Scand J Trauma Resusc Emerg Med. 2012 Apr 11;20:30. doi: 10.1186/1757-7241-20-30. PMID: 22494785. Exclusion: Ineligible intervention.

447. Rose WD, Anderson LD, Edmond SA. Analysis of intubations. Before and after establishment of a rapid sequence intubation protocol for air medical use. Air Med J. 1994 Nov-Dec;13(11-12):475-8. doi: 10.1016/S1067-991X(05)80289-5. PMID: 10137495. Exclusion: Ineligible intervention.

448. Rowe BH. Review: prehospital noninvasive ventilation for severe respiratory distress reduces hospital mortality. Ann Intern Med. 2014 May;160(10):JC2. doi: 10.7326/00034819-160-10-201405200-02002. PMID: 24842436. Exclusion: Ineligible publication type.

449. Rubens AJ, Stoy W, Piane G. Using interactive videodisc to test advanced airway management skills. Prehospital Disaster Med. 1995 Oct-Dec;10(4):251-8. doi: 10.1017/s1049023x00042126. PMID: 10155437. Exclusion: Ineligible population.

450. Rubenson Wahlin R, Nelson DW, Bellander $\mathrm{BM}$, et al. Prehospital intubation and outcome in traumatic brain injury-assessing intervention efficacy in a modern trauma cohort. Front Neurol. 2018 Apr 10;9:194. doi: 10.3389/fneur.2018.00194. PMID: 29692755. Exclusion: Ineligible comparison or no comparison.
451. Ruchholtz S, Waydhas C, Ose C, et al. Prehospital intubation in severe thoracic trauma without respiratory insufficiency: a matched-pair analysis based on the Trauma Registry of the German Trauma Society. J Trauma. 2002 May;52(5):879-86. doi: 10.1097/00005373-200205000-00010. PMID: 11988653. Exclusion: Ineligible comparison or no comparison.

452. Rupprecht H, Heppner HJ, Wohlfart K, et al. The geriatric polytrauma: risk profile and prognostic factors. Ulus Travma Acil Cerrahi Derg. 2017 Mar;23(2):156-62. doi: 10.5505/tjtes.2016.77177. PMID: 28467584. Exclusion: Ineligible comparison or no comparison.

453. Russi CS, Myers LA, Kolb LJ, et al. The Airtraq Optical Laryngoscope in helicopter emergency medical services: a pilot trial. Air Med J. 2013 Mar-Apr;32(2):88-92. doi: 10.1016/j.amj.2012.06.013. PMID: 23452367. Exclusion: KQ4 Triage.

454. Russo SG, Becke K. Expected difficult airway in children. Curr Opin Anaesthesiol. 2015 Jun;28(3):321-6. doi: 10.1097/ACO.0000000000000198. PMID: 25887198. Exclusion: Ineligible publication type.

455. Russo SG, Nickel EA, Leissner KB, et al. Use of the GlideScope-Ranger for prehospital intubations by anaesthesia trained emergency physicians - an observational study. BMC Emerg Med. 2016 Jan 29;16:8. doi: 10.1186/s12873-016-0069-2. PMID: 26830474. Exclusion: Ineligible comparison or no comparison.

456. Russo SG, Zink W, Herff H, et al. [Death due to (no) airway. Adverse events by outof-hospital airway management?]. Anaesthesist. 2010 Oct;59(10):929-39. doi: 10.1007/s00101-010-1782-y. PMID: 20827450. Exclusion: Not in English, but may be relevant.

457. Ryynanen OP, Iirola T, Reitala J, et al. Is advanced life support better than basic life support in prehospital care? A systematic review. Scand J Trauma Resusc Emerg Med. 2010 Nov 23;18:62. doi: 10.1186/17577241-18-62. PMID: 21092256. Exclusion: Systematic review used to identify primary studies. 
458. Sagarin MJ, Barton ED, Chng YM, et al. Airway management by US and Canadian emergency medicine residents: a multicenter analysis of more than 6,000 endotracheal intubation attempts. Ann Emerg Med. 2005 Oct;46(4):328-36. doi: 10.1016/j.annemergmed.2005.01.009. PMID: 16187466. Exclusion: Studies that meet other criteria but setting is in Emergency department.

459. Sagarin MJ, Barton ED, Sakles JC, et al. Underdosing of midazolam in emergency endotracheal intubation. Acad Emerg Med. 2003 Apr;10(4):329-38. doi: 10.1197/aemj.10.4.329. PMID: 12670846. Exclusion: Ineligible intervention.

460. Saissy JM. Simplified use of mixed propofol and alfentanil for anesthesia in remote locations. Mil Med. 2000 Mar;165(3):195-9. PMID: 10741082. Exclusion: Ineligible setting.

461. Sakurai A, Kinoshita K, Maeda Y, et al. Confirmed cardiac output on emergency medical services arrival as confounding by indication: an observational study of prehospital airway management in patients with out-of-hospital cardiac arrest. Emerg Med J. 2019 Jul;36(7):410-5. doi: 10.1136/emermed-2018-208107. PMID: 31171627. Exclusion: Ineligible comparison or no comparison.

462. Sams J, M. KA. Use of an emergency sedation protocol to assist intubation in helicopter patient retrieval in Victoria. Emerg Med Australas. 1999;11(2):84-9. Exclusion: Ineligible comparison or no comparison.

463. Sandroni C, De Santis P, D'Arrigo S. Capnography during cardiac arrest. Resuscitation. 2018 Nov;132:73-7. doi: 10.1016/j.resuscitation.2018.08.018. PMID: 30142399. Exclusion: Ineligible intervention.

464. Sanfilippo F, Chiarenza F, Maybauer DM, et al. The Easytube for airway management: a systematic review of clinical and simulation studies. J Clin Anesth. 2016 Jun;31:215-22. doi: 10.1016/j.jclinane.2016.01.039. PMID: 27185715. Exclusion: Ineligible population.

465. Sanson G, Di Bartolomeo S, Nardi G, et al. Road traffic accidents with vehicular entrapment: incidence of major injuries and need for advanced life support. Eur J Emerg Med. 1999 Dec;6(4):285-91. doi: 10.1097/00063110-199912000-00002. PMID: 10646914. Exclusion: Ineligible comparison or no comparison.
466. Santos D, Mitchell R. The history of pediatric airway reconstruction. Laryngoscope. 2010 Apr;120(4):815-20. doi: 10.1002/lary.20823. PMID: 20205174. Exclusion: Ineligible publication type.

467. Sasada MP, Gabbott DA. The role of the laryngeal mask airway in pre-hospital care. Resuscitation. 1994 Oct;28(2):97-102. doi: 10.1016/0300-9572(94)90079-5. PMID: 7846379. Exclusion: Ineligible publication type.

468. Savino PB, Reichelderfer S, Mercer MP, et al. Direct versus video laryngoscopy for prehospital intubation: a systematic review and meta-analysis. Acad Emerg Med. 2017 Aug;24(8):1018-26. doi:

10.1111/acem.13193. PMID: 28370736. Exclusion: Systematic review used to identify primary studies.

469. Sayre MR, Sackles JC, Mistler AF, et al. Field trial of endotracheal intubation by basic EMTs. Ann Emerg Med. 1998 Feb;31(2):228-33. PMID: 28139992. Exclusion: Ineligible comparison or no comparison.

470. Schalk R, Byhahn C, Fausel F, et al. Out-ofhospital airway management by paramedics and emergency physicians using laryngeal tubes. Resuscitation. 2010 Mar;81(3):323-6. doi: 10.1016/j.resuscitation.2009.11.007. PMID: 20006418. Exclusion: KQ4 Triage.

471. Schalk R, Meininger D, Ruesseler M, et al. Emergency airway management in trauma patients using laryngeal tube suction. Prehosp Emerg Care. 2011 JulSep;15(3):347-50. doi: 10.3109/10903127.2011.561405. PMID: 21521037. Exclusion: KQ4 Triage.

472. Schalk R, Seeger FH, Mutlak H, et al. Complications associated with the prehospital use of laryngeal tubes--a systematic analysis of risk factors and strategies for prevention. Resuscitation. 2014 Nov;85(11):1629-32. doi: 10.1016/j.resuscitation.2014.07.014. PMID: 25110247. Exclusion: Ineligible comparison or no comparison.

473. Schaller RJ, Huff JS, Zahn A. Comparison of a colorimetric end-tidal $\mathrm{CO} 2$ detector and an esophageal aspiration device for verifying endotracheal tube placement in the prehospital setting: a six-month experience. Prehospital Disaster Med. 1997 JanMar;12(1):57-63. doi: 10.1017/s1049023x00037237. PMID: 10166376. Exclusion: Ineligible intervention. 
474. Schmidbauer W, Ahlers O, Spies C, et al. Early prehospital use of non-invasive ventilation improves acute respiratory failure in acute exacerbation of chronic obstructive pulmonary disease. Emerg Med J. 2011 Jul;28(7):626-7. doi: 10.1136/emj.2009.089102. PMID: 20844095. Exclusion: Ineligible intervention.

475. Schmidt AR, Ulrich L, Seifert B, et al. Ease and difficulty of pre-hospital airway management in 425 paediatric patients treated by a helicopter emergency medical service: a retrospective analysis. Scand J Trauma Resusc Emerg Med. 2016 Mar 05;24:22. doi: 10.1186/s13049-016-0212-9. PMID: 26944389. Exclusion: Ineligible comparison or no comparison.

476. Schyma B, Gobindram A, Joseph A, et al. Advanced airway interventions in the trapped (racing) driver: a randomised crossover trial. British Paramedic Journal. 2017;2(2):2-12. Exclusion: Ineligible population.

477. Seamon MJ, Doane SM, Gaughan JP, et al. Prehospital interventions for penetrating trauma victims: a prospective comparison between advanced life support and basic life support. Injury. 2013 May;44(5):634-8. doi: 10.1016/j.injury.2012.12.020. PMID: 23391450. Exclusion: Ineligible population.

478. Selde W, English K, Heffelfinger M, et al. Successful airtraq use in an air medical transport system. Air Med J. 2014 NovDec;33(6):331-4. doi: 10.1016/j.amj.2014.07.003. PMID: 25441532. Exclusion: Ineligible setting.

479. Sharma V. First-pass success rate between rocuronium and succinylcholine in emergent out-of-hospital endotracheal intubation. Critical Care Alert. 2020;28(5):1-3. Exclusion: Ineligible publication type.

480. Shavit I, Aviram E, Hoffmann Y, et al. Laryngeal mask airway as a rescue device for failed endotracheal intubation during scene-to-hospital air transport of combat casualties. Eur J Emerg Med. 2018 Oct;25(5):368-71. doi: 10.1097/MEJ.0000000000000480. PMID: 28657971. Exclusion: Ineligible comparison or no comparison.

481. Shy BD, Rea TD, Becker LJ, et al. Time to intubation and survival in prehospital cardiac arrest. Prehosp Emerg Care. 2004 Oct-Dec;8(4):394-9. doi: 10.1016/j.prehos.2004.06.013. PMID: 15626000. Exclusion: Ineligible comparison or no comparison.
482. Sibley A, Mackenzie M, Bawden J, et al. A prospective review of the use of ketamine to facilitate endotracheal intubation in the helicopter emergency medical services (HEMS) setting. Emerg Med J. 2011 Jun;28(6):521-6. doi: 10.1136/emj.2009.088237. PMID: 20926628. Exclusion: Ineligible comparison or no comparison.

483. Silvestri S, Ralls GA, Krauss B, et al. The effectiveness of out-of-hospital use of continuous end-tidal carbon dioxide monitoring on the rate of unrecognized misplaced intubation within a regional emergency medical services system. Ann Emerg Med. 2005 May;45(5):497-503. doi: 10.1016/j.annemergmed.2004.09.014. PMID: 15855946. Exclusion: Ineligible intervention.

484. Sim SS, Lien WC, Chou HC, et al. Ultrasonographic lung sliding sign in confirming proper endotracheal intubation during emergency intubation. Resuscitation. 2012 Mar;83(3):307-12. doi:

10.1016/j.resuscitation.2011.11.010. PMID: 22138058. Exclusion: Ineligible intervention.

485. Simpson PM, Bendall JC. Prehospital noninvasive ventilation for acute cardiogenic pulmonary oedema: an evidence-based review. Emerg Med J. 2011 Jul;28(7):60912. doi: 10.1136/emj.2010.092296. PMID: 21076052. Exclusion: Ineligible intervention.

486. Sing RF, Reilly PM, Rotondo MF, et al. Out-of-hospital rapid-sequence induction for intubation of the pediatric patient. Acad Emerg Med. 1996 Jan;3(1):41-5. doi: 10.1111/j.1553-2712.1996.tb03301.x. PMID: 8749966. Exclusion: Ineligible comparison or no comparison.

487. Sing RF, Rotondo MF, Zonies DH, et al. Rapid sequence induction for intubation by an aeromedical transport team: a critical analysis. Am J Emerg Med. 1998 Oct;16(6):598-602. doi: 10.1016/s07356757(98)90227-3. PMID: 9786546. Exclusion: Ineligible comparison or no comparison.

488. Sirbaugh PE, Pepe PE, Shook JE, et al. A prospective, population-based study of the demographics, epidemiology, management, and outcome of out-of-hospital pediatric cardiopulmonary arrest. Ann Emerg Med. 1999 Feb;33(2):174-84. doi: 0.1016/s01960644(99)70391-4. PMID: 9922413. Exclusion: Ineligible intervention. 
489. Slagt C, Zondervan A, Patka P, et al. A retrospective analysis of the intubations performed during 5 years of helicopter emergency medical service in Amsterdam. Air Med J. 2004 Sep-Oct;23(5):36-7. doi: 10.1016/j.amj.2004.06.004. PMID: 15337954. Exclusion: Ineligible comparison or no comparison.

490. Slater EA, Weiss SJ, Ernst AA, et al. Preflight versus en route success and complications of rapid sequence intubation in an air medical service. J Trauma. 1998 Sep;45(3):588-92. doi: 10.1097/00005373199809000-00031. PMID: 9751556. Exclusion: KQ4 Triage.

491. Smith CE, Kovach B, Polk JD, et al. Prehospital tracheal intubating conditions during rapid sequence intubation: rocuronium versus vecuronium. Air Med J. 2002 Jan-Feb;21(1):26-32. doi: 10.1067/mmj.2002.121713. PMID: 11805764. Exclusion: KQ4 Triage.

492. Smith KA, Gothard MD, Schwartz HP, et al. Risk factors for failed tracheal intubation in pediatric and neonatal critical care specialty transport. Prehosp Emerg Care. 2015 JanMar;19(1):17-22. doi: 10.3109/10903127.2014.964888. PMID: 25350689. Exclusion: Ineligible setting.

493. Sollid SJ, Lossius HM, Soreide E. Prehospital intubation by anaesthesiologists in patients with severe trauma: an audit of a Norwegian helicopter emergency medical service. Scand J Trauma Resusc Emerg Med. 2010 Jun 14;18:30. doi: 10.1186/17577241-18-30. PMID: 20546578. Exclusion: Ineligible comparison or no comparison.

494. Sonday CJ, Axelband J, Jacoby J, et al. Thiopental vs. etomidate for rapid sequence intubation in aeromedicine. Prehospital Disaster Med. 2005 Sep-Oct;20(5):324-6. doi: 10.1017/s1049023x00002788. PMID: 16295169. Exclusion: KQ4 Triage.

495. Sonne A, Wulffeld S, Steinmetz J, et al. Prehospital interventions before and after implementation of a physician-staffed helicopter. Dan Med J. 2017 Oct;64(10) PMID: 28975883. Exclusion: Ineligible intervention.

496. Speer T, Dersch W, Kleine B, et al. Mechanical ventilation during resuscitation: how manual chest compressions affect a ventilator's function. Adv Ther. 2017 Oct;34(10):2333-44. doi: 10.1007/s12325017-0615-7. PMID: 28983829. Exclusion: Ineligible population.
497. Stassen W, Lithgow A, Wylie C, et al. A descriptive analysis of endotracheal intubation in a South African helicopter emergency medical service. Afr J Emerg Med. 2018 Dec;8(4):140-4. doi: 10.1016/j.afjem.2018.07.002. PMID: 30534517. Exclusion: Ineligible comparison or no comparison.

498. Staudinger T, Brugger S, Roggla M, et al. Comparison of the Combitube with the endotracheal tube in cardiopulmonary resuscitation in the prehospital phase. German. Wien Klin Wochenschr. 1994;13:412-5. PMID: 8091765. Exclusion: Not in English, but may be relevant.

499. Sternbach GL, Varon J, Fromm RE, et al. Galen and the origins of artificial ventilation, the arteries and the pulse. Resuscitation. 2001 May;49(2):119-22. doi: 10.1016/s0300-9572(01)00344-6. PMID: 11382516. Exclusion: Ineligible publication type.

500. Stevenson AG, Graham CA, Hall R, et al. Tracheal intubation in the emergency department: the Scottish district hospital perspective. Emerg Med J. 2007 Jun;24(6):394-7. doi: 10.1136/emj.2006.041988. PMID: 17513533. Exclusion: Studies that meet other criteria but setting is in Emergency department.

501. Stevenson C, Baker K, Dhindsa HS. Use of the endotracheal tube introducer to improve intubation success rate...2010 Critical Care Transport Medicine Conference held April 12-14, 2010, San Antonio, Texas. Air Med J. 2010;29(4):163-. Exclusion: Ineligible publication type.

502. Stiell IG, Nesbitt LP, Pickett W, et al. The OPALS Major Trauma Study: impact of advanced life-support on survival and morbidity. Cmaj. 2008 Apr 22;178(9):114152. doi: 10.1503/cmaj.071154. PMID: 18427089. Exclusion: Ineligible comparison or no comparison.

503. Stiell IG, Spaite DW, Field B, et al. Advanced life support for out-of-hospital respiratory distress. N Engl J Med. 2007 May 24;356(21):2156-64. doi: 10.1056/NEJMoa060334. PMID: 17522399. Exclusion: Ineligible intervention.

504. Stiell IG, Wells GA, Field B, et al. Advanced cardiac life support in out-ofhospital cardiac arrest. N Engl J Med. 2004 Aug 12;351(7):647-56. doi: 10.1056/NEJMoa040325. PMID: 15306666. Exclusion: Ineligible intervention. 
505. Stone CK, Thomas SH. Is oral endotracheal intubation efficacy impaired in the helicopter environment? Air Med J. 1994 Aug;13(8):319-21. doi: 10.1016/S1067991X(05)80337-2. PMID: 10135926.

Exclusion: Ineligible population.

506. Stratton SJ, Underwood LA, Whalen SM, et al. Prehospital pediatric endotracheal intubation: a survey of the United States. Prehospital Disaster Med. 1993 OctDec;8(4):323-6. doi: 10.1017/s1049023x00040589. PMID: 10146431. Exclusion: Ineligible intervention.

507. Struck MF, Wittrock M, Nowak A. Prehospital Glidescope video laryngoscopy for difficult airway management in a helicopter rescue program with anaesthetists. Eur J Emerg Med. 2011 Oct;18(5):282-4. doi: 10.1097/MEJ.0b013e328344e70f. PMID: 21430543. Exclusion: Ineligible comparison or no comparison.

508. Su E, Mann NC, McCall M, et al. Use of resuscitation skills by paramedics caring for critically injured children in Oregon.

Prehosp Emerg Care. 1997 Jul-

Sep;1(3):123-7. doi:

10.1080/10903129708958803. PMID:

9709352. Exclusion: Ineligible outcome.

509. Subramanian A, Garcia-Marcinkiewicz AG, Brown DR, et al. Definitive airway management of patients presenting with a pre-hospital inserted King LT(S)-D laryngeal tube airway: a historical cohort study. Can J Anaesth. 2016 Mar;63(3):27582. doi: 10.1007/s12630-015-0493-x. PMID: 26514982. Exclusion: Ineligible intervention.

510. Sunde GA, Brattebo G, Odegarden T, et al. Laryngeal tube use in out-of-hospital cardiac arrest by paramedics in Norway. Scand $\mathrm{J}$ Trauma Resusc Emerg Med. 2012 Dec 18;20:84. doi: 10.1186/1757-7241-20-84. PMID: 23249522. Exclusion: Ineligible comparison or no comparison.

511. Sunde GA, Sandberg M, Lyon R, et al. Hypoxia and hypotension in patients intubated by physician staffed helicopter emergency medical services - a prospective observational multi-centre study. BMC Emerg Med. 2017 Jul 11;17(1):22. doi: 10.1186/s12873-017-0134-5. PMID: 28693491. Exclusion: KQ4 Triage.
512. Swanson ER, Fosnocht DE. Effect of an airway education program on prehospital intubation. Air Med J. 2002 JulAug;21(4):28-31. doi: 10.1067/mmj.2002.125936. PMID: 12087321. Exclusion: KQ4 Triage.

513. Swanson ER, Fosnocht DE, Jensen SC. Comparison of etomidate and midazolam for prehospital rapid-sequence intubation. Prehosp Emerg Care. 2004 JulSep;8(3):273-9. doi: 10.1016/j.prehos.2003.12.026. PMID: 15295727. Exclusion: KQ4 Triage.

514. Swanson ER, Fosnocht DE, Neff RJ. The use of etomidate for rapid-sequence intubation in the air medical setting. Prehosp Emerg Care. 2001 Apr-Jun;5(2):142-6. doi: 10.1080/10903120190940001. PMID: 11339723. Exclusion: Ineligible comparison or no comparison.

515. Syverud SA, Borron SW, Storer DL, et al. Prehospital use of neuromuscular blocking agents in a helicopter ambulance program. Ann Emerg Med. 1988 Mar;17(3):236-42. doi: 10.1016/s0196-0644(88)80114-8. PMID: 3345016. Exclusion: Ineligible publication date.

516. Taghavi S, Vora HP, Jayarajan SN, et al. Prehospital intubation does not decrease complications in the penetrating trauma patient. Am Surg. 2014 Jan;80(1):9-14. PMID: 24401498. Exclusion: Ineligible comparison or no comparison.

517. Takeda T, Tanigawa K, Tanaka H, et al. The assessment of three methods to verify tracheal tube placement in the emergency setting. Resuscitation. 2003 Feb;56(2):1537. doi: 10.1016/s0300-9572(02)00345-3. PMID: 12589988. Exclusion: Studies that meet other criteria but setting is in Emergency department.

518. Tam RK, Maloney J, Gaboury I, et al. Review of endotracheal intubations by Ottawa advanced care paramedics in Canada. Prehosp Emerg Care. 2009 JulSep;13(3):311-5. doi: 10.1080/10903120902935231. PMID: 19499466. Exclusion: Ineligible intervention.

519. Tan CK, Ko SC, Huang HC, et al. Tracheal perforation after tracheal intubation. Resuscitation. 2007 May;73(2):174-5. doi: 10.1016/j.resuscitation.2006.10.006. PMID: 17291671. Exclusion: Ineligible study design. 
520. Tanigawa K, Shigematsu A. Choice of airway devices for 12,020 cases of nontraumatic cardiac arrest in Japan. Prehosp Emerg Care. 1998 Apr-Jun;2(2):96100. doi: 10.1080/10903129808958850. PMID: 9709326. Exclusion: Ineligible outcome.

521. Tanigawa K, Takeda $\mathrm{T}$, Goto E, et al. Accuracy and reliability of the self-inflating bulb to verify tracheal intubation in out-ofhospital cardiac arrest patients. Anesthesiology. 2000 Dec;93(6):1432-6. doi: 10.1097/00000542-200012000-00015. PMID: 11149438. Exclusion: Ineligible intervention.

522. Tarpgaard M, Hansen TM, Rognas L. Anaesthetist-provided pre-hospital advanced airway management in children: a descriptive study. Scand J Trauma Resusc Emerg Med. 2015 Aug 27;23:61. doi: 10.1186/s13049-015-0140-0. PMID: 26307040. Exclusion: Ineligible comparison or no comparison.

523. Taylor DM, Bernard SA, Masci K, et al. Prehospital noninvasive ventilation: a viable treatment option in the urban setting. Prehosp Emerg Care. 2008 Jan-

Mar;12(1):42-5. doi: 10.1080/10903120701710389. PMID: 18189176. Exclusion: Ineligible comparison or no comparison.

524. Taylor J, Black S, S JB, et al. Design and implementation of the AIRWAYS-2 trial: a multi-centre cluster randomised controlled trial of the clinical and cost effectiveness of the i-gel supraglottic airway device versus tracheal intubation in the initial airway management of out of hospital cardiac arrest. Resuscitation. 2016 12;109:25-32. doi: 10.1016/j.resuscitation.2016.09.016. PMID: 27697605. Exclusion: Ineligible study design.

525. Tekwani KL, Watts HF, Chan CW, et al. The effect of single-bolus etomidate on septic patient mortality: a retrospective review. West J Emerg Med. 2008 Nov;9(4):195-200. PMID: 19561744. Exclusion: Ineligible population.

526. Tentillier E, Heydenreich C, Cros AM, et al. Use of the intubating laryngeal mask airway in emergency pre-hospital difficult intubation. Resuscitation. 2008 Apr;77(1):30-4. doi: 10.1016/j.resuscitation.2007.06.035. PMID: 18022753. Exclusion: Ineligible comparison or no comparison.
527. Thibodeau LG, Verdile VP, Bartfield JM. Incidence of aspiration after urgent intubation. Am J Emerg Med. 1997 Oct;15(6):562-5. doi: 10.1016/s07356757(97)90157-1. PMID: 9337361. Exclusion: Studies that meet other criteria but setting is in Emergency department.

528. Thierbach AR, Piepho T, Maybauer M. The EasyTube for airway management in emergencies. Prehosp Emerg Care. 2005 Oct-Dec;9(4):445-8. doi: 10.1080/10903120500254910. PMID: 16263680. Exclusion: Ineligible comparison or no comparison.

529. Thoeni N, Piegeler T, Brueesch M, et al. Incidence of difficult airway situations during prehospital airway management by emergency physicians--a retrospective analysis of 692 consecutive patients. Resuscitation. 2015 May;90:42-5. doi: 10.1016/j.resuscitation.2015.02.010. PMID: 25708959. Exclusion: Ineligible outcome.

530. Thomas SH, Harrison T, Wedel SK. Flight crew airway management in four settings: a six-year review. Prehosp Emerg Care. 1999 Oct-Dec;3(4):310-5. doi: 10.1080/10903129908958960. PMID: 10534031. Exclusion: KQ4 Triage.

531. Thomas S, Judge T, Lowell MJ, et al. Airway management success and hypoxemia rates in air and ground critical care transport: a prospective multicenter study. Prehosp Emerg Care. 2010 JulSep;14(3):283. doi: 10.3109/10903127.2010.481758. PMID: 20507218. Exclusion: Ineligible comparison or no comparison.

532. Thompson CB, Balasz K, Goltermann J, et al. Intubation quality assurance thresholds. Air Med J. 1995 Apr-Jun;14(2):55-60. doi: 10.1016/s1067-991x(95)90095-0. PMID: 10143544. Exclusion: Ineligible intervention.

533. Tiah L, Kajino K, Alsakaf O, et al. Does pre-hospital endotracheal intubation improve survival in adults with nontraumatic out-of-hospital cardiac arrest? A systematic review. West J Emerg Med. 2014 Nov;15(7):749-57. doi: 10.5811/westjem.2014.9.20291. PMID: 25493114. Exclusion: Systematic review used to identify primary studies. 
534. Tiamfook-Morgan TO, Harrison TH, Thomas SH. What happens to $\mathrm{SpO} 2$ during air medical crew intubations? Prehosp Emerg Care. 2006 Jul-Sep;10(3):363-8. doi: 10.1080/10903120600725835. PMID: 16801281. Exclusion: Ineligible comparison or no comparison.

535. Tijssen JA, Prince DK, Morrison LJ, et al. Time on the scene and interventions are associated with improved survival in pediatric out-of-hospital cardiac arrest. Resuscitation. 2015 Sep;94:1-7. doi: 10.1016/j.resuscitation.2015.06.012. PMID: 26095301. Exclusion: Ineligible comparison or no comparison.

536. Timmermann A, Eich C, Russo SG, et al. Prehospital airway management: a prospective evaluation of anaesthesia trained emergency physicians. Resuscitation. 2006 Aug;70(2):179-85. doi:

10.1016/j.resuscitation.2006.01.010. PMID: 16828956. Exclusion: Ineligible outcome.

537. Timmermann A, Russo SG, Eich C, et al. The out-of-hospital esophageal and endobronchial intubations performed by emergency physicians. Anesth Analg. 2007 Mar;104(3):619-23. doi: 10.1213/01.ane.0000253523.80050.e9. PMID: 17312220. Exclusion: Ineligible comparison or no comparison.

538. Timmermann A, Russo SG, Rosenblatt WH, et al. Intubating laryngeal mask airway for difficult out-of-hospital airway management: a prospective evaluation. $\mathrm{Br} \mathrm{J}$ Anaesth. 2007 Aug;99(2):286-91. doi: 10.1093/bja/aem136. PMID: 17584849. Exclusion: Ineligible comparison or no comparison.

539. Tollefsen WW, Brown CA, 3rd, Cox KL, et al. Two hundred sixty pediatric emergency airway encounters by air transport personnel: a report of the air transport emergency airway management (NEAR VI: "A-TEAM") project. Pediatr Emerg Care. 2013 Sep;29(9):963-8. doi: 10.1097/PEC.0b013e3182a219ea. PMID: 23974713. Exclusion: Ineligible comparison or no comparison.

540. Tollefsen WW, Chapman J, Frakes M, et al. Endotracheal tube cuff pressures in pediatric patients intubated before aeromedical transport. Pediatr Emerg Care. 2010 May;26(5):361-3. doi: 10.1097/PEC.0b013e3181db224d. PMID: 20404779. Exclusion: Ineligible outcome.
541. Tracy S, Schinco MA, Griffen MM, et al. Urgent airway intervention: does outcome change with personnel performing the procedure? J Trauma. 2006 Nov;61(5):11625. doi: 10.1097/01.ta.0000243887.90697.06. PMID: 17099523. Exclusion: Ineligible comparison or no comparison.

542. Trakulsrichai S, Sundarathiti P, Chalermdamrichai P, et al. An observation study of rapid sequence, awake and sedation-only intubations in an emergency department in Thai patients. J Med Assoc Thai. 2009 Aug;92(8):1022-7. PMID: 19694325. Exclusion: Studies that meet other criteria but setting is in Emergency department.

543. Tran DT, Newton EK, Mount VA, et al. Rocuronium versus succinylcholine for rapid sequence induction intubation. Cochrane Database Syst Rev. 2015 Oct 29(10):Cd002788. doi: 10.1002/14651858.CD002788.pub3. PMID: 26512948. Exclusion: Ineligible setting.

544. Tritsch L, Boet S, Pottecher J, et al. Intubating laryngeal mask airway placement by non-physician healthcare providers in management out-of-hospital cardiac arrests: a case series. Resuscitation. 2014 Mar;85(3):320-5. doi: 10.1016/j.resuscitation.2013.11.006. PMID: 24287330. Exclusion: Ineligible study design.

545. Truhlar A, Ferson DZ. Use of the laryngeal mask airway supreme in pre-hospital difficult airway management. Resuscitation. 2008 Aug;78(2):107-8. doi: 10.1016/j.resuscitation.2008.03.008. PMID: 18508181. Exclusion: Ineligible publication type.

546. Tsur AM, Nadler R, Tsur N, et al. Prehospital definitive airway is not associated with improved survival in trauma patients. J Trauma Acute Care Surg. 2020 Aug;89(2S Suppl 2):S237-S41. doi: 10.1097/TA.0000000000002722. PMID: 32301876. Exclusion: Ineligible comparison or no comparison.

547. Tuma M, El-Menyar A, Abdelrahman H, et al. Prehospital intubation in patients with isolated severe traumatic brain injury: a 4year observational study. Crit Care Res Pract. 2014;2014:135986. doi: 10.1155/2014/135986. PMID: 24527211. Exclusion: KQ4 Triage. 
548. Tweed J, George T, Greenwell C, et al. Prehospital airway management examined at two pediatric emergency centers. Prehospital Disaster Med. 2018 Oct;33(5):532-8. doi: 10.1017/S1049023X18000882. PMID: 30379129. Exclusion: Ineligible comparison or no comparison.

549. Tyrode A. Airway management in the multi trauma patient. Australian Emergency Nursing Journal. 1997;1(3):6-9. Exclusion: Ineligible study design.

550. Ufberg JW, Bushra JS, Karras DJ, et al. Aspiration of gastric contents: association with prehospital intubation. Am J Emerg Med. 2005 May;23(3):379-82. doi: 10.1016/j.ajem.2005.02.005. PMID: 15915418. Exclusion: Ineligible comparison or no comparison.

551. Vadeboncoeur TF, Davis DP, Ochs M, et al. The ability of paramedics to predict aspiration in patients undergoing prehospital rapid sequence intubation. J Emerg Med. 2006 Feb;30(2):131-6. doi: 10.1016/j.jemermed.2005.04.019. PMID: 16567245. Exclusion: Ineligible outcome.

552. van Exter P. Prehospital endotracheal intubation in patients with severe traumatic brain injury: guidelines versus reality. Resuscitation. 2010 May;81(5):627; author reply -8. doi:

10.1016/j.resuscitation.2009.10.028. PMID: 20398847. Exclusion: Ineligible publication type.

553. van Tulder R, Schriefl C, Roth D, et al. Laryngeal tube practice in a metropolitan ambulance service: a five-year retrospective observational study (2009-2013). Prehosp Emerg Care. 2016 Apr 26;24(3):434-40. doi: 10.3109/10903127.2015.1129473. PMID: 27115936. Exclusion: Ineligible outcome.

554. Vandromme MJ, Melton SM, Griffin R, et al. Intubation patterns and outcomes in patients with computed tomography-verified traumatic brain injury. J Trauma. 2011 Dec;71(6):1615-9. doi:

10.1097/TA.0b013e31822a30a1. PMID: 21841511. Exclusion: Ineligible comparison or no comparison.

555. Varga S, Shupp JW, Maher D, et al. Trauma airway management: transition from anesthesia to emergency medicine. J Emerg Med. 2013 Jun;44(6):1190-5. doi: 10.1016/j.jemermed.2012.11.074. PMID: 23473818. Exclusion: Studies that meet other criteria but setting is in Emergency department.
556. Velmahos GC, Vassiliu P, Chan LS, et al. Influence of flail chest on outcome among patients with severe thoracic cage trauma. Int Surg. 2002 Oct-Dec;87(4):240-4. PMID: 12575808. Exclusion: Ineligible comparison or no comparison.

557. Vezina MC, Trepanier CA, Nicole PC, et al. Complications associated with the Esophageal-Tracheal Combitube in the prehospital setting. Can J Anaesth. 2007 Feb;54(2):124-8. doi: 10.1007/BF03022008. PMID: 17272251. Exclusion: Ineligible outcome.

558. Vijayakumar E, Bosscher H, Renzi FP, et al. The use of neuromuscular blocking agents in the emergency department to facilitate tracheal intubation in the trauma patient: help or hindrance? J Crit Care. 1998 Mar;13(1):1-6. doi: 10.1016/s08839441(98)90022-3. PMID: 9556120. Exclusion: Studies that meet other criteria but setting is in Emergency department.

559. Vilke GM, Steen PJ, Smith AM, et al. Outof-hospital pediatric intubation by paramedics: the San Diego experience. J Emerg Med. 2002 Jan;22(1):71-4. doi: 10.1016/s0736-4679(01)00439-5. PMID: 11809559. Exclusion: Ineligible comparison or no comparison.

560. Vincent-Lambert C, Makkink A, Kloppers F. Keep pushing! Limiting interruptions to CPR; bag-valve mask versus i-gel ${ }^{\circledR}$ airway ventilation. Health SA Gesondheid. 2016;21(1):21-32. Exclusion: Ineligible intervention.

561. Vithalani VD, Vlk S, Davis SQ, et al. Unrecognized failed airway management using a supraglottic airway device. Resuscitation. 2017 Oct;119:1-4. doi: 10.1016/j.resuscitation.2017.07.019. PMID: 28750882. Exclusion: Ineligible comparison or no comparison.

562. Vohra TT, Miller JB, Nicholas KS, et al. Endotracheal intubation in patients treated for prehospital status epilepticus. Neurocrit Care. 2015 Aug;23(1):33-43. doi: 10.1007/s12028-014-0106-5. PMID: 25623785. Exclusion: Ineligible comparison or no comparison.

563. von Elm E, Schoettker P, Henzi I, et al. Prehospital tracheal intubation in patients with traumatic brain injury: systematic review of current evidence. Br J Anaesth. 2009 Sep;103(3):371-86. doi: 10.1093/bja/aep202. PMID: 19648153. Exclusion: Systematic review used to identify primary studies. 
564. von Vopelius-Feldt J, Wood J, Benger J. Critical care paramedics: where is the evidence? A systematic review. Emerg Med J. 2014 Dec;31(12):1016-24. doi: 10.1136/emermed-2013-202721. PMID: 24071949. Exclusion: Systematic review used to identify primary studies.

565. Voss S, Rhys M, Coates D, et al. How do paramedics manage the airway during out of hospital cardiac arrest? Resuscitation. 2014 Dec;85(12):1662-6. doi: 10.1016/j.resuscitation.2014.09.008. PMID: 25260723. Exclusion: Ineligible comparison or no comparison.

566. Wadhwa R, Kalra S. Comparison of hemodynamic changes and ease of endotracheal intubation through I-gel vs. ILMA. J Anaesthesiol Clin Pharmacol. 2010;26(3):379-82. Exclusion: Ineligible setting.

567. Walker RG, White LJ, Whitmore GN, et al. Evaluation of physiologic alterations during prehospital paramedic-performed rapid sequence intubation. Prehosp Emerg Care. 2018 May-Jun;22(3):300-11. doi: 10.1080/10903127.2017.1380095. PMID: 29297718. Exclusion: Ineligible comparison or no comparison.

568. Walrath BD, Harper S, Barnard E, et al. Airway management for trauma patients. Mil Med. 2018 Sep 01;183(suppl 2):29-31. doi: 10.1093/milmed/usy124. PMID: 30189067. Exclusion: Ineligible study design.

569. Wang $\mathrm{CH}$, Lee AF, Chang WT, et al. Comparing effectiveness of initial airway interventions for out-of-hospital cardiac arrest: a systematic review and network meta-analysis of clinical controlled trials. Ann Emerg Med. 2020 May;75(5):627-36. doi: 10.1016/j.annemergmed.2019.12.003. PMID: 31983493. Exclusion: Systematic review used to identify primary studies.

570. Wang HE, Balasubramani GK, Cook LJ, et al. Medical conditions associated with outof-hospital endotracheal intubation. Prehosp Emerg Care. 2011a Jul-Sep;15(3):338-46. doi: 10.3109/10903127.2011.569850. PMID: 21612386. Exclusion: Ineligible comparison or no comparison.
571. Wang HE, Brown SP, MacDonald RD, et al. Association of out-of-hospital advanced airway management with outcomes after traumatic brain injury and hemorrhagic shock in the ROC hypertonic saline trial. Emerg Med J. 2014 Mar;31(3):186-91. doi: 10.1136/emermed-2012-202101. PMID: 23353663. Exclusion: Ineligible comparison or no comparison.

572. Wang HE, Cook LJ, Chang CC, et al. Outcomes after out-of-hospital endotracheal intubation errors. Resuscitation. 2009 Jan;80(1):50-5. doi:

10.1016/j.resuscitation.2008.08.016. PMID: 18952357. Exclusion: Ineligible comparison or no comparison.

573. Wang HE, Davis DP, O'Connor RE, et al. Drug-assisted intubation in the prehospital setting (resource document to NAEMSP position statement). Prehosp Emerg Care. 2006c Apr-Jun;10(2):261-71. doi: 10.1080/10903120500541506. PMID: 16531387. Exclusion: Systematic review used to identify primary studies.

574. Wang HE, Davis DP, Wayne MA, et al. Prehospital rapid-sequence intubation--what does the evidence show? Proceedings from the 2004 National Association of EMS Physicians annual meeting. Prehosp Emerg Care. 2004a Oct-Dec;8(4):366-77. PMID: 15625996. Exclusion: Ineligible study design.

575. Wang HE, Donnelly JP, Barton D, et al. Assessing advanced airway management performance in a national cohort of emergency medical services agencies. Ann Emerg Med. 2018a May;71(5):597-607.e3. doi: 10.1016/j.annemergmed.2017.12.012. PMID: 29352616. Exclusion: Ineligible comparison or no comparison.

576. Wang HE, Kupas DF, Paris PM, et al. Multivariate predictors of failed prehospital endotracheal intubation. Acad Emerg Med. 2003a Jul;10(7):717-24. doi: 10.1111/j.1553-2712.2003.tb00065.x. PMID: 12837645. Exclusion: Ineligible comparison or no comparison.

577. Wang HE, Kupas DF, Paris PM, et al. Preliminary experience with a prospective, multi-centered evaluation of out-of-hospital endotracheal intubation. Resuscitation. 2003b Jul;58(1):49-58. doi: 10.1016/s03009572(03)00058-3. PMID: 12867309. Exclusion: Ineligible outcome. 
578. Wang HE, Kupas DF, Paris PM, et al. Factors associated with the use of pharmacologic agents to facilitate out-ofhospital endotracheal intubation. Prehosp Emerg Care. 2004d Jan-Mar;8(1):1-9. doi: 10.1080/312703002740. PMID: 14691780. Exclusion: Ineligible outcome.

579. Wang HE, Lave JR, Sirio CA, et al. Paramedic intubation errors: isolated events or symptoms of larger problems? Health Aff (Millwood). 2006a Mar-Apr;25(2):501-9. doi: 10.1377/hlthaff.25.2.501. PMID: 16522604. Exclusion: Ineligible comparison or no comparison.

580. Wang HE, Mann NC, Mears G, et al. Outof-hospital airway management in the United States. Resuscitation. 2011b Apr;82(4):378-85. doi: 10.1016/j.resuscitation.2010.12.014. PMID: 21288624. Exclusion: Ineligible comparison or no comparison. - excluded per email from Tamr

581. Wang HE, O'Connor RE, Domeier RM, et al. Prehospital rapid-sequence intubation. Prehosp Emerg Care. 2001b JanMar;5(1):40-8. PMID: 11194068. Exclusion: Ineligible study design.

582. Wang HE, O'Connor RE, Megargel RE, et al. The utilization of midazolam as a pharmacologic adjunct to endotracheal intubation by paramedics. Prehosp Emerg Care. 2000 Jan-Mar;4(1):14-8. doi: 10.1080/10903120090941560. PMID: 10634276. Exclusion: Ineligible comparison or no comparison.

583. Wang HE, O'Connor RE, Schnyder ME, et al. Patient status and time to intubation in the assessment of prehospital intubation performance. Prehosp Emerg Care. 2001a Jan-Mar;5(1):10-8. doi: 10.1080/10903120190940254. PMID: 11194061. Exclusion: Ineligible intervention.

584. Wang HE, Peitzman AB, Cassidy LD, et al. Out-of-hospital endotracheal intubation and outcome after traumatic brain injury. Ann Emerg Med. 2004b Nov;44(5):439-50. doi: 10.1016/j.annemergmed.2004.04.008. PMID: 15520702. Exclusion: Ineligible comparison or no comparison.

585. Wang HE, Prince DK, Stephens SW, et al. Design and implementation of the Resuscitation Outcomes Consortium Pragmatic Airway Resuscitation Trial (PART). Resuscitation. 2016 Apr;101:5764. doi: 10.1016/j.resuscitation.2016.01.012. PMID: 26851059. Exclusion: Ineligible publication type.
586. Wang HE, Seitz SR, Hostler D, et al. Defining the learning curve for paramedic student endotracheal intubation. Prehosp Emerg Care. 2005 Apr-Jun;9(2):156-62. doi: 10.1080/10903120590924645. PMID: 16036839. Exclusion: Ineligible intervention.

587. Wang HE, Sternig K, Benger J, et al. Intubation on trial: randomized trials of prehospital endotracheal intubation in cardiac arrest will influence how we intubate patients J Emerg Med Serv JEMS. 2015 Aug;40(8):40-2, 4. PMID: 26403044. Exclusion: Ineligible publication type.

588. Wang HE, Sweeney TA, O'Connor RE, et al. Failed prehospital intubations: an analysis of emergency department courses and outcomes. Prehosp Emerg Care. 2001c Apr-Jun;5(2):134-41. doi: 10.1080/10903120190939995. PMID: 11339722. Exclusion: Ineligible comparison or no comparison.

589. Warner KJ, Carlbom D, Cooke CR, et al Paramedic training for proficient prehospital endotracheal intubation. Prehosp Emerg Care. 2010 Jan-Mar;14(1):103-8. doi: 10.3109/10903120903144858. PMID: 19947874. Exclusion: KQ4 Triage.

590. Warner KJ, Cuschieri J, Copass MK, et al. The impact of prehospital ventilation on outcome after severe traumatic brain injury. J Trauma. 2007 Jun;62(6):1330-6; discussion 6-8. doi: 10.1097/TA.0b013e31804a8032. PMID: 17563643. Exclusion: Ineligible comparison or no comparison.

591. Warner KJ, Cuschieri J, Jurkovich GJ, et al. Single-dose etomidate for rapid sequence intubation may impact outcome after severe injury. J Trauma. 2009a Jul;67(1):45-50. doi: 10.1097/TA.0b013e3181a92a70. PMID: 19590307. Exclusion: KQ4 Triage.

592. Warner KJ, Sharar SR, Copass MK, et al. Prehospital management of the difficult airway: a prospective cohort study. J Emerg Med. 2009b Apr;36(3):257-65. doi: 10.1016/j.jemermed.2007.10.058. PMID: 18439793. Exclusion: Ineligible comparison or no comparison.

593. Wayne MA, Friedland E. Prehospital use of succinylcholine: a 20-year review. Prehosp Emerg Care. 1999 Apr-Jun;3(2):107-9. doi: 10.1080/10903129908958916. PMID: 10225641. Exclusion: Ineligible study design. 
594. Weingart GS, Carlson JN, Callaway CW, et al. Estimates of sedation in patients undergoing endotracheal intubation in US EDs. Am J Emerg Med. 2013 Jan;31(1):2226. doi: 10.1016/j.ajem.2012.05.015. PMID: 22770915. Exclusion: Ineligible intervention.

595. Weinstein EP, Sayre MR, Mistler AF, et al. Endotracheal intubation by basic EMTs...'Field trial of endotracheal intubation by basic EMTs' by Sayre et al. Ann Emerg Med. 1998;32(3 part 1):391-2. Exclusion: Ineligible publication type.

596. Welsford M. Management of out-of-hospital cardiac arrest with laryngeal tube improved survival vs endotracheal intubation. APC Journal Club. 2019;34(1):JC4-JC. PMID: 30641555. Exclusion: Ineligible study design.

597. Werman HA, Schwegman D, Gerard JP. The effect of etomidate on airway management practices of an air medical transport service. Prehosp Emerg Care. 2004 Apr-Jun;8(2):185-90. doi: 10.1016/j.prehos.2003.12.012. PMID: 15060854. Exclusion: KQ4 Triage.

598. Wesley K, Wesley K. Is intubation success rate higher with two-medic crews. JEMS. 2017;42(1):24-5. PMID: 29206400. Exclusion: Ineligible study design.

599. West JR, Lott C, Donner L, et al. Periintubation factors affecting emergency physician choice of paralytic agent for rapid sequence intubation of trauma patients. Am J Emerg Med. 2018 Jul;36(7):1151-4. doi: 10.1016/j.ajem.2017.11.038. PMID: 29162438. Exclusion: Ineligible outcome.

600. White L, Melhuish T, Holyoak R, et al. Advanced airway management in out of hospital cardiac arrest: a systematic review and meta-analysis. Am J Emerg Med. 2018 Dec;36(12):2298-306. doi:

10.1016/j.ajem.2018.09.045. PMID: 30293843. Exclusion: Systematic review used to identify primary studies.

601. Whitley A. Pre-hospital providers use of ketamine for rapid sequence intubation. PreHospital Providers Use of Ketamine for Rapid Sequence Intubation. 2017:1-. Exclusion: Ineligible outcome.
602. Wiese CH, Semmel T, Muller JU, et al. The use of the laryngeal tube disposable (LT-D) by paramedics during out-of-hospital resuscitation-an observational study concerning ERC guidelines 2005. Resuscitation. 2009 Feb;80(2):194-8. doi: 10.1016/j.resuscitation.2008.08.023. PMID: 19010582. Exclusion: KQ4 Triage.

603. Wilbers NE, Hamaekers AE, Jansen J, et al. Prehospital airway management: a prospective case study. Acta Anaesthesiol Belg. 2011;62(1):23-31. PMID: 21612142. Exclusion: Ineligible comparison or no comparison.

604. Wimalasena Y, Burns B, Reid C, et al. Apneic oxygenation was associated with decreased desaturation rates during rapid sequence intubation by an Australian helicopter emergency medicine service. Ann Emerg Med. 2015 Apr;65(4):371-6. doi: 10.1016/j.annemergmed.2014.11.014. PMID: 25536868. Exclusion: KQ4 Triage.

605. Winchell RJ, Hoyt DB. Endotracheal intubation in the field improves survival in patients with severe head injury. Trauma Research and Education Foundation of San Diego. Arch Surg. 1997 Jun;132(6):592-7. doi: 10.1001/archsurg.1997.01430300034007. PMID: 9197850. Exclusion: Ineligible comparison or no comparison.

606. Wirtz DD, Ortiz C, Newman DH, et al. Unrecognized misplacement of endotracheal tubes by ground prehospital providers. Prehosp Emerg Care. 2007 AprJun;11(2):213-8. doi: 10.1080/10903120701205935. PMID: 17454811. Exclusion: Ineligible study design.

607. Wnent J, Franz R, Seewald S, et al. Difficult intubation and outcome after out-of-hospital cardiac arrest: a registry-based analysis. Scand J Trauma Resusc Emerg Med. 2015 Jun 06;23:43. doi: 10.1186/s13049-0150124-0. PMID: 26048574. Exclusion: Ineligible comparison or no comparison.

608. Writer H. Cardiorespiratory arrest in children (out of hospital). Clin Evid (Online). 2007 Sep 01;01:01. PMID: 19450304. Exclusion: Systematic review used to identify primary studies.

609. Writer H. Cardiorespiratory arrest in children (out of hospital). Clin Evid (Online). 2010 Nov 25;25:25. PMID: 21406131. Exclusion: Systematic review used to identify primary studies. 
610. Yoo JY, Chae YJ, Park SY, et al. Time to tracheal intubation over a fibreoptic bronchoscope using a silicone left doublelumen endobronchial tube versus polyvinyl chloride single-lumen tube with bronchial blocker: a randomized controlled noninferiority trial. J Thorac Dis. 2019 Mar;11(3):901-8. doi: 10.21037/jtd.2019.01.108. PMID: 31019779. Exclusion: Ineligible outcome.

611. Young B. The intubating laryngeal-mask airway may be an ideal device for airway control in the rural trauma patient. Am J Emerg Med. 2003 Jan;21(1):80-5. doi: 10.1053/ajem.2003.50012. PMID: 12563589. Exclusion: Ineligible comparison or no comparison.

612. Youngquist S, Gausche-Hill M, Burbulys D. Alternative airway devices for use in children requiring prehospital airway management: update and case discussion. Pediatr Emerg Care. 2007 Apr;23(4):250-8; quiz 9-61. doi: 10.1097/PEC.0b013e31803f7552. PMID: 17438442. Exclusion: Ineligible publication type.

613. Yun MJ, Hwang JW, Park SH, et al. The 90degree rotation technique improves the ease of insertion of the ProSealTM laryngeal mask airway in children. Can J Anaesth. 2011 Apr;58(4):379-83. doi: 10.1007/s12630-010-9452-8. PMID: 21203877. Exclusion: Ineligible setting.

614. Zechner PM, Breitkreutz R. Ultrasound instead of capnometry for confirming tracheal tube placement in an emergency? Resuscitation. 2011 Oct;82(10):1259-61. doi: 10.1016/j.resuscitation.2011.06.040. PMID: 21763250. Exclusion: Ineligible intervention.

615. Zed PJ, Abu-Laban RB, Harrison DW. Intubating conditions and hemodynamic effects of etomidate for rapid sequence intubation in the emergency department: an observational cohort study. Acad Emerg Med. 2006 Apr;13(4):378-83. doi: 10.1197/j.aem.2005.11.076. PMID: 16531603. Exclusion: Studies that meet other criteria but setting is in Emergency department.

616. Zettervall SL, Sirajuddin S, Akst S, et al. Use of propofol as an induction agent in the acutely injured patient. Eur J Trauma Emerg Surg. 2015 Aug;41(4):405-11. doi: 10.1007/s00068-014-0479-3. PMID: 26038005. Exclusion: Studies that meet other criteria but setting is in Emergency department.
617. Zuckerbraun NS, Pitetti RD, Herr SM, et al. Use of etomidate as an induction agent for rapid sequence intubation in a pediatric emergency department. Acad Emerg Med. 2006 Jun;13(6):602-9. doi: 10.1197/j.aem.2005.12.026. PMID: 16636355. Exclusion: Studies that meet other criteria but setting is in Emergency department. 


\section{Appendix E. Study Characteristics Evidence Table}

Shown in associated Excel ${ }^{\circledR}$ file. 


\section{Appendix F. Outcomes Evidence Table}

Shown in associated Excel ${ }^{\circledR}$ file. 


\section{Appendix G. Risk of Bias}

Table G-1. Risk of bias for randomized controlled trials (part 1 of 2)

\begin{tabular}{|c|c|c|c|c|c|c|c|c|}
\hline Author, Year & $\begin{array}{c}\text { Key } \\
\text { Question }\end{array}$ & $\begin{array}{c}\text { 1. Was } \\
\text { Randomization } \\
\text { Adequate? }\end{array}$ & $\begin{array}{l}\text { 2. Was the } \\
\text { Allocation of } \\
\text { Treatment } \\
\text { Adequately } \\
\text { Concealed? }\end{array}$ & $\begin{array}{l}\text { 3. Were Groups } \\
\text { Similar at } \\
\text { Baseline? }\end{array}$ & $\begin{array}{l}\text { 4. Was Analysis } \\
\text { Intent to Treat? }\end{array}$ & $\begin{array}{l}\text { 5. Did Analyses } \\
\text { Include All Eligible } \\
\text { Trial Participants } \\
\text { Postrandomization } \\
\text { (i.e., Were Not } \\
\text { Excluded)? }\end{array}$ & $\begin{array}{c}\text { 6. Was Loss } \\
\text { to } \\
\text { Followup or } \\
\text { Missing Data } \\
20 \% \\
\text { or Less? }\end{array}$ & $\begin{array}{l}\text { 7. Were } \\
\text { Comparable } \\
\text { Groups } \\
\text { Maintained? }\end{array}$ \\
\hline Arima, 2014 & 4 & Unclear & Yes & No & Yes & No & Yes & Yes \\
\hline Benger, 2016 & 4 & Yes & Yes & Yes & Yes & Yes & Yes & Yes \\
\hline Benger, 2018 & 3 & Yes & Yes & Yes & Yes & Yes & Yes & Yes \\
\hline Chan, 2020a & 4 & Yes & NR & Yes & Yes & Yes & Yes & Yes \\
\hline Ducharme, 2017 & 4 & Unclear & Unclear & Unclear & Yes & Yes & Yes & Yes \\
\hline Fiala, 2017 & 1 & NR & Yes & Yes & Yes & Yes & Yes & Yes \\
\hline Frascone, 2011 & 3 & Yes & No & Yes & Yes & No & Yes & Yes \\
\hline Jabre, 2011 & 4 & Yes & Yes & Yes & Yes & Yes & Yes & Yes \\
\hline Jabre, 2018 & 2 & Yes & Yes & Yes & Yes & Yes & Yes & Yes \\
\hline Khosravan, 2015 & 3 & Yes & Unclear & Yes & No & Yes & Yes & Yes \\
\hline Kreutziger, 2019 & 4 & Yes & Yes & Yes & Yes & Yes & Yes & Yes \\
\hline Macke, 2020 & 4 & Yes & Yes & Yes & Yes & Yes & Yes & Yes \\
\hline Middleton, 2014 & 4 & Yes & No & Yes & Yes & Yes & No & Unclear \\
\hline Ono, 2014 & 4 & Unclear & No & Yes & Yes & No & Yes & Yes \\
\hline Rabitsch, 2003 & 3 & No & No & Yes & Unclear & Yes & Yes & Yes \\
\hline Rumball, 1997 & 1,4 & Yes & Yes & Yes & Yes & Yes & Unclear & $\begin{array}{l}\text { Yes for success, no } \\
\text { for Pco2 and } \mathrm{Po} 2\end{array}$ \\
\hline Trimmel, 2011 & 4 & Yes & No & Yes & No & Yes & Yes & Yes \\
\hline Trimmel, 2016 & 4 & Yes & No & Yes & Yes & Yes & Yes & Yes \\
\hline $\begin{array}{l}\text { Wang, } 2018 \\
\text { Lupton, } 2020\end{array}$ & $1,2,3$ & Yes & NR & Unclear & Yes & Yes & Yes & Yes \\
\hline
\end{tabular}


Table G-2. Risk of bias for randomized controlled trials (part 2 of 2)

\begin{tabular}{|c|c|c|c|c|c|c|c|}
\hline Author, Year & $\begin{array}{c}\text { Key } \\
\text { Question }\end{array}$ & $\begin{array}{l}\text { 8. Were Outcomes } \\
\text { Assessed Using } \\
\text { Valid and Reliable } \\
\text { Measures? }\end{array}$ & $\begin{array}{c}\text { 9. Was Outcome } \\
\text { Measurement or } \\
\text { Ascertainment Similar } \\
\text { Between Groups? }\end{array}$ & $\begin{array}{c}\text { 10. Were Outcome } \\
\text { Assessors Blinded, or Were } \\
\text { Outcomes Objectively } \\
\text { Measured? }\end{array}$ & $\begin{array}{l}\text { 11. Were Outcomes } \\
\text { Prespecified and Were } \\
\text { Primary/Prespecified } \\
\text { Outcomes Reported? }\end{array}$ & $\begin{array}{c}\text { Was the } \\
\text { Study } \\
\text { Registered? }\end{array}$ & $\begin{array}{l}\text { Risk of Bias } \\
\text { Quality Rating }\end{array}$ \\
\hline Arima, 2014 & 4 & Yes & Yes & Yes & Yes & NR & Moderate \\
\hline Benger, 2016 & 4 & Yes & Yes & $\begin{array}{l}\text { Yes for clinical outcomes, no } \\
\text { for process outcomes }\end{array}$ & Yes & Yes & $\begin{array}{l}\text { Low for clinical outcomes, } \\
\text { moderate for process } \\
\text { outcomes }\end{array}$ \\
\hline Benger, 2018 & 3 & Yes & Yes & Yes & Yes & Yes & Low \\
\hline Bernard, 2010 & 2 & Yes & Yes & Yes & Yes & NR & Low \\
\hline Chan, 2020a & 4 & $\begin{array}{l}\text { Success: Unclear } \\
\text { ROSC: Yes }\end{array}$ & Yes & $\begin{array}{l}\text { Success: No } \\
\text { ROSC: Yes }\end{array}$ & Yes & No & $\begin{array}{l}\text { Success: Moderate } \\
\text { ROSC: Low }\end{array}$ \\
\hline Ducharme, 2017 & 4 & Yes & Yes & Unclear & Yes & NR & High \\
\hline \begin{tabular}{|l|} 
Fiala, 2017 \\
\end{tabular} & 1 & Unclear & Yes & No & Yes & Yes & Moderate \\
\hline Frascone, 2011 & 3 & Yes & Yes & No & Yes & NR & Moderate \\
\hline Jabre, 2011 & 4 & Yes & Yes & Yes & Yes & NR & Low \\
\hline Jabre, 2018 & 2 & Yes & Yes & Yes & Yes & Yes & Low \\
\hline Khosravan, 2015 & 3 & Yes & Yes & Yes & Yes & Yes & Moderate \\
\hline Kreutziger, 2019 & 4 & Yes & Unclear & Unclear & Yes & NR & Moderate \\
\hline Malinverni, 2019 & 2 & NR & NR & NR & NR & Yes & Moderate \\
\hline \begin{tabular}{|l|} 
Middleton, 2014 \\
\end{tabular} & 4 & Yes & Yes & Yes & Yes & Yes & High \\
\hline Ono, 2014 & 4 & Unclear & Yes & $\begin{array}{l}\text { NR for CPC, unclear for } \\
\text { success. }\end{array}$ & Yes & NR & $\begin{array}{l}\text { Moderate for success, } \\
\text { survival, and ROSC, high } \\
\text { for CPC. }\end{array}$ \\
\hline Rabitsch, 2003 & 3 & Yes & Yes & Yes & Yes & NR & Low \\
\hline Rumball, 1997 & 1,4 & $\begin{array}{l}\text { No for success, yes } \\
\text { for blood gasses }\end{array}$ & Yes & $\begin{array}{l}\text { No for success, yes for blood } \\
\text { gasses }\end{array}$ & Yes & NR & Moderate \\
\hline Trimmel, 2011 & 4 & Unclear & Yes & $\begin{array}{l}\text { No blinding. Unclear if } \\
\text { provider confirmed own } \\
\text { placement. }\end{array}$ & Yes & NR & High \\
\hline Trimmel, 2016 & 4 & Yes & Yes & No blinding & Yes & NR & Moderate \\
\hline $\begin{array}{l}\text { Wang, } 2018 \\
\text { Lupton, } 2020\end{array}$ & $1,2,3$ & Yes & Yes & $\begin{array}{l}\text { Yes for survival and ROSC, } \\
\text { blinding NR for Modified } \\
\text { Rankin Scale, no for success }\end{array}$ & Yes & Yes & $\begin{array}{l}\text { Low for Survival and ROSC, } \\
\text { moderate for Modified } \\
\text { Rankin Scale, and success }\end{array}$ \\
\hline
\end{tabular}

$\mathrm{CPC}=$ Cerebral Performance Category Score; $\mathrm{NR}=$ not reported, $\mathrm{ROSC}=$ return of spontaneous circulation 
Table G-3. Risk of bias for controlled clinical trials

\begin{tabular}{|c|c|c|c|c|c|c|c|c|c|c|c|c|c|}
\hline $\begin{array}{l}\text { Author, } \\
\text { Year }\end{array}$ & $\begin{array}{c}\text { Key } \\
\text { Question }\end{array}$ & $\begin{array}{l}\text { 1. Was the } \\
\text { Group } \\
\text { Allocation } \\
\text { Protocol } \\
\text { Sufficient To } \\
\text { Minimize } \\
\text { Group } \\
\text { Differences? }\end{array}$ & $\begin{array}{l}\text { 2. Was the } \\
\text { Allocation } \\
\text { of } \\
\text { Treatment } \\
\text { Adequately } \\
\text { Concealed? }\end{array}$ & $\begin{array}{c}\text { 3. Were } \\
\text { Groups } \\
\text { Similar at } \\
\text { Base- } \\
\text { line? }\end{array}$ & $\begin{array}{c}\text { 4. Did } \\
\text { Analyses } \\
\text { Include All } \\
\text { Eligible Trial } \\
\text { Participants } \\
\text { After Group } \\
\text { Allocation (i.e., } \\
\text { Were Not } \\
\text { Excluded)? }\end{array}$ & $\begin{array}{l}\text { 5. Was } \\
\text { Loss to } \\
\text { Follow- } \\
\text { up or } \\
\text { Missing } \\
\text { Data } \\
20 \% \\
\text { or Less? }\end{array}$ & $\begin{array}{l}\text { 6. Was } \\
\text { There } \\
\text { Minimal } \\
\text { Differen- } \\
\text { tial Loss } \\
\text { to } \\
\text { Followup } \\
\text { or Missing } \\
\text { Data? }\end{array}$ & $\begin{array}{c}\text { 7. Were } \\
\text { Outcomes } \\
\text { Assessed } \\
\text { Using } \\
\text { Valid and } \\
\text { Reliable } \\
\text { Measures? }\end{array}$ & $\begin{array}{l}\text { 8. Was } \\
\text { Measurement } \\
\text { or Ascertain- } \\
\text { ment Similar } \\
\text { Between } \\
\text { Groups? }\end{array}$ & $\begin{array}{c}\text { 9. Were } \\
\text { Outcome } \\
\text { Assessors } \\
\text { Blinded, or } \\
\text { Were } \\
\text { Outcomes } \\
\text { Objectively } \\
\text { Measured? }\end{array}$ & $\begin{array}{c}\text { 10. Were } \\
\text { Outcomes } \\
\text { Pre- } \\
\text { Specified } \\
\text { and Were } \\
\text { Primaryl } \\
\text { Pre- } \\
\text { Specified } \\
\text { Outcomes } \\
\text { Reported? }\end{array}$ & $\begin{array}{c}\text { Was the } \\
\text { Study } \\
\text { Registered } \\
?\end{array}$ & $\begin{array}{l}\text { Risk of } \\
\text { Bias } \\
\text { Quality } \\
\text { Rating }\end{array}$ \\
\hline \begin{tabular}{|l} 
Bartlett, \\
1992 \\
\end{tabular} & 3 & No & No & Yes & No & No & No & Yes & Yes & Yes & Yes & No & High \\
\hline $\begin{array}{l}\text { Bozeman, } \\
2006 \\
\end{array}$ & 4 & Yes & Unclear & Yes & Yes & Yes & Yes & Yes & Yes & Yes & Yes & No & Moderate \\
\hline $\begin{array}{l}\text { Gausche, } \\
2000\end{array}$ & 2,4 & Yes & NA & Yes & Yes & Yes & Yes & $\begin{array}{l}\text { Yes for } \\
\text { mortality, } \\
\text { unclear for } \\
\text { function }\end{array}$ & Yes & $\begin{array}{l}\text { Yes for } \\
\text { mortality, } \\
\text { unclear for } \\
\text { PCPC }\end{array}$ & Yes & No & $\begin{array}{l}\text { Low for } \\
\text { mortality, } \\
\text { moderate } \\
\text { for PCPC }\end{array}$ \\
\hline $\begin{array}{l}\text { Maignan, } \\
2015\end{array}$ & 1 & Yes & No & Yes & Yes & Yes & Yes & Yes & Yes & Yes & Yes & Yes & Moderate \\
\hline \begin{tabular}{|l|} 
Rumball, \\
2004 \\
\end{tabular} & 3 & Unclear & No & No & Yes & Yes & Yes & Yes & Yes & Yes & Yes & No & Moderate \\
\hline $\begin{array}{l}\text { Sos-Kanto, } \\
2009\end{array}$ & 1 & NR & NR & Yes & Yes & Yes & Yes & Yes & Yes & Yes & Yes & No & Low \\
\hline
\end{tabular}

$\mathrm{NA}=$ not applicable, $\mathrm{NR}=$ not reported, $\mathrm{PCPC}=$ pediatric cerebral performance category 
Table G-4. Risk of bias for prospective cohort studies

\begin{tabular}{|c|c|c|c|c|c|c|c|c|c|c|}
\hline Author, Year & $\begin{array}{c}\text { Key } \\
\text { Question }\end{array}$ & \begin{tabular}{|c|} 
1. Did the \\
Study Attempt \\
To Enroll or \\
Include All or \\
a Random \\
Sample of \\
Patients \\
Meeting \\
Inclusion \\
Criteria? \\
\end{tabular} & $\begin{array}{l}\text { 2. Were the } \\
\text { Groups Similar } \\
\text { at Baseline, or } \\
\text { Did the Design } \\
\text { or Analysis } \\
\text { Account for } \\
\text { Important } \\
\text { Confounding } \\
\text { and Modifying } \\
\text { Variables? }\end{array}$ & $\begin{array}{c}\text { 3. Was Loss } \\
\text { to } \\
\text { Followup or } \\
\text { Missing Data } \\
20 \% \\
\text { or Less? } \\
\end{array}$ & \begin{tabular}{|c|} 
4. Were \\
Comparable \\
Groups \\
Maintained? \\
(Was There \\
Minimal \\
Differential \\
Loss to \\
Followup or \\
Missing Data?) \\
\end{tabular} & $\begin{array}{c}\text { 5. Were } \\
\text { Outcomes } \\
\text { Prespecified } \\
\text { and Were } \\
\text { Primary/ } \\
\text { Prespecified } \\
\text { Outcomes } \\
\text { Reported? }\end{array}$ & $\begin{array}{c}\text { 6. Were } \\
\text { Outcomes } \\
\text { Assessed } \\
\text { Using Valid } \\
\text { and Reliable } \\
\text { Measures? }\end{array}$ & $\begin{array}{c}\text { 7. Were } \\
\text { Outcome } \\
\text { Assessors } \\
\text { Blinded, or } \\
\text { Were } \\
\text { Outcomes } \\
\text { Objectively } \\
\text { Measured? }\end{array}$ & $\begin{array}{c}\text { Was the } \\
\text { Study } \\
\text { (Protocol) } \\
\text { Registered } \\
?\end{array}$ & $\begin{array}{l}\text { Risk of Bias } \\
\text { Quality Rating }\end{array}$ \\
\hline Breeman, 2020 & 4 & Yes & Unclear & Yes & Unclear & Yes & Yes & Yes & No & Moderate \\
\hline Eich, 2009 & 4 & Yes & No & Yes & Yes & Yes & Yes & No & No & Moderate \\
\hline Hankins, 1993 & 3 & Yes & Unclear/NR & Yes & Unclear & Yes & No & No & No & High \\
\hline Hansen, 2020 & $1,2,3$ & Yes & No & Yes & No & Yes & Yes & $\begin{array}{l}\text { Yes survival, } \\
\text { no success }\end{array}$ & No & Moderate \\
\hline Hiltunen, 2016 & 3 & Yes & Yes & Yes & Unclear & Yes & Yes & Yes & Yes & $\begin{array}{l}\text { Moderate for } \\
\text { Success Rate }\end{array}$ \\
\hline \begin{tabular}{|l|} 
Jarman, 2017 \\
\end{tabular} & 3,4 & Yes & No & No & Yes & Yes & \begin{tabular}{|l|} 
No for first- \\
pass success, \\
yes for ROSC. \\
\end{tabular} & Yes & No & Moderate \\
\hline Kajino, 2011 & 3 & Yes & Yes & Yes & Yes & Yes & Yes & $\begin{array}{l}\text { Yes for ROSC } \\
\text { and Survival } \\
\text { NR for CPC } \\
\end{array}$ & No & $\begin{array}{l}\text { Low for ROSC } \\
\text { and survival, high } \\
\text { for CPC. }\end{array}$ \\
\hline McCall, 2008 & 3 & Yes & Unclear & NA & Yes & Yes & Yes & Yes & No & Moderate \\
\hline McMahan, 1992 & 3 & Yes & Yes & NA & Yes & Yes & Yes & Yes & No & Low \\
\hline Myers, 2016 & 4 & Yes & Unclear & Yes & Yes & Yes & Unclear & No & No & High \\
\hline Prekker, 2014 & 4 & Yes & Yes & Yes & $\mathrm{N} / \mathrm{A}$ & Yes & Yes & No & No & Moderate \\
\hline Risse, 2020 & 4 & Yes & Unclear & Yes & Unclear & Yes & Yes & Yes & No & Moderate \\
\hline Roth, 2015 & 1 & Yes & Yes & Yes & Yes & Yes & Yes & Yes & No & Low \\
\hline $\begin{array}{l}\text { Sulzgruber, } \\
2018\end{array}$ & $1,2,3$ & Yes & Yes & Yes & Yes & Yes & Yes & Unclear & NR & $\begin{array}{l}\text { Low for Mortality } \\
\text { and ROSC, } \\
\text { moderate for CPC }\end{array}$ \\
\hline Sunde, 2015 & 4 & Yes & Yes & Yes & Yes & Yes & Yes & No & Yes & Moderate \\
\hline Takei, 2010 & $1,2,3$ & Yes & No & Unclear & $\begin{array}{l}\text { Yes for short- } \\
\text { term outcomes, } \\
\text { unclear for } 1- \\
\text { month survival. }\end{array}$ & Yes & Yes & Yes & NR & $\begin{array}{l}\text { Moderate for } \\
\text { short-term } \\
\text { outcomes, high } \\
\text { for 1-month } \\
\text { survival } \\
\end{array}$ \\
\hline
\end{tabular}

$\mathrm{CPC}=$ cerebral performance category, $\mathrm{NA}=$ not applicable, $\mathrm{NR}=$ not reported, $\mathrm{ROSC}=$ return of spontaneous circulation 
Table G-5. Risk of bias for before/after study designs

\begin{tabular}{|c|c|c|c|c|c|c|c|c|c|c|c|}
\hline Author, Year & $\begin{array}{c}\text { Key } \\
\text { Question }\end{array}$ & \begin{tabular}{|c|} 
1. Did the \\
Study \\
Attempt To \\
Enroll or \\
Include All or \\
a Random \\
Sample of \\
Patients \\
Meeting \\
Inclusion \\
Criteria?
\end{tabular} & $\begin{array}{l}\text { 2. Were the } \\
\text { Groups Similar } \\
\text { at Baseline, or } \\
\text { Did the Design } \\
\text { or Analysis } \\
\text { Account for } \\
\text { Important } \\
\text { Confounding } \\
\text { and Modifying } \\
\text { Variables? }\end{array}$ & $\begin{array}{c}\text { 3. Were } \\
\text { Groups } \\
\text { Comparable } \\
\text { Across Time } \\
\text { Periods? }\end{array}$ & $\begin{array}{l}\text { 4. Were } \\
\text { Outcome } \\
\text { Assessors } \\
\text { Blinded, or } \\
\text { Were } \\
\text { Outcomes } \\
\text { Objectively } \\
\text { Measured? }\end{array}$ & $\begin{array}{c}5 . \text { Was } \\
\text { Loss to } \\
\text { Followup or } \\
\text { Missing } \\
\text { Data } 20 \% \\
\text { or Less? }\end{array}$ & $\begin{array}{c}\text { 6. Were } \\
\text { Comparable } \\
\text { Groups } \\
\text { Maintained? }\end{array}$ & $\begin{array}{c}\text { 7. Were } \\
\text { Outcomes } \\
\text { Assessed } \\
\text { Using Valid } \\
\text { and } \\
\text { Reliable } \\
\text { Measures? }\end{array}$ & $\begin{array}{c}\text { 8. Were } \\
\text { Outcomes } \\
\text { Prespecified } \\
\text { and Were } \\
\text { Primaryl } \\
\text { Prespecified } \\
\text { Outcomes } \\
\text { Reported? }\end{array}$ & $\begin{array}{c}\text { Was the } \\
\text { Study } \\
\text { (Protocol) } \\
\text { Registered? }\end{array}$ & $\begin{array}{c}\text { Risk of } \\
\text { Bias } \\
\text { Quality } \\
\text { Rating }\end{array}$ \\
\hline Chien, 2012 & 1 & Yes & Yes & Yes & Yes & No & Unclear & Yes & Yes & No & Moderate \\
\hline \begin{tabular}{|l} 
Dos Santos, \\
2011
\end{tabular} & 4 & Unclear & Unclear & Unclear & No & Yes & NA & Yes & Yes & No & High \\
\hline Frascone, 2013 & Harms & $\begin{array}{l}\text { Unclear - not } \\
\text { all eligible } \\
\text { providers } \\
\text { agreed to } \\
\text { participate }\end{array}$ & No & Unclear & No & Yes & Unclear & $\begin{array}{l}\text { No for } \\
\text { Success. } \\
\text { Yes for } \\
\text { Harms }\end{array}$ & Yes & No & High \\
\hline Gahan, 2011 & 3 & Yes & Yes & Unclear & $\begin{array}{l}\text { Yes for harms, } \\
\text { no for success }\end{array}$ & Yes & Yes & Yes & Yes & No & Low \\
\hline Jabre, 2007 & 4 & Yes & Yes & Yes & Yes & Yes & Yes & Yes & Yes & No & Low \\
\hline \begin{tabular}{|l|} 
Jarvis, 2015 \\
\end{tabular} & 4 & Yes & Yes & No & Yes & Yes & Yes & Yes & Yes & No & Moderate \\
\hline Louka, 2018 & 4 & Yes & Unclear & No & Unclear & Yes & Unclear & Unclear & Yes & No & High \\
\hline Wayne, 2010 & 4 & Yes & Yes & Yes & Unclear & Yes & Yes & Yes & Yes & No & Moderate \\
\hline
\end{tabular}

$\mathrm{NA}=$ not applicable

\section{Table G-6. Risk of bias for retrospective studies}

\begin{tabular}{|c|c|c|c|c|c|c|c|c|}
\hline Author, Year & $\begin{array}{c}\text { Key } \\
\text { Question }\end{array}$ & $\begin{array}{l}\text { 1. Was the } \\
\text { Selection of } \\
\text { Patients for } \\
\text { Inclusion } \\
\text { Unbiased? }\end{array}$ & $\begin{array}{l}\text { 2. Were the } \\
\text { Groups } \\
\text { Similar at } \\
\text { Baseline, or } \\
\text { Did the Design } \\
\text { or Analysis } \\
\text { Account for } \\
\text { Important } \\
\text { Confounding } \\
\text { and Modifying } \\
\text { Variables? }\end{array}$ & $\begin{array}{l}\text { 3. Were Outcome Assessors } \\
\text { Blinded, or Were Outcomes } \\
\text { Objectively Measured? }\end{array}$ & $\begin{array}{l}\text { 4. Were } \\
\text { Outcomes } \\
\text { Assessed } \\
\text { Using Valid } \\
\text { and Reliable } \\
\text { Measures? }\end{array}$ & \begin{tabular}{|c|} 
5. Were \\
Outcomes Pre- \\
specified and \\
Were Primaryl \\
Prespecified \\
Outcomes \\
Reported?
\end{tabular} & $\begin{array}{l}\text { 6. Was the } \\
\text { Study } \\
\text { (Protocol) } \\
\text { Registered? }\end{array}$ & Risk of Bias Quality Rating \\
\hline Becker, 2018 & 3 & Yes & No & Yes & Yes & Yes & No & High \\
\hline Behrens, 2020 & 3 & Yes & Yes & $\begin{array}{l}\text { Yes: mortality, ROSC } \\
\text { No: neurological function (CPC) }\end{array}$ & Yes & Yes & No & $\begin{array}{l}\text { Low: mortality, ROSC } \\
\text { Moderate: neurological function }\end{array}$ \\
\hline Bendinelli, 2018 & 4 & Yes & Yes & $\begin{array}{l}\text { Yes: mortality, length of stay } \\
\text { No: success }\end{array}$ & $\begin{array}{l}\text { Yes: mortality, } \\
\text { length of stay } \\
\text { No: success }\end{array}$ & Yes & No & $\begin{array}{l}\text { Low: mortality, length of stay } \\
\text { Moderate: success }\end{array}$ \\
\hline Bulger, 2005 & 4 & Yes & Yes & Yes & $\begin{array}{l}\text { Yes (not using } \\
\text { GCS) }\end{array}$ & Yes & No & Low \\
\hline
\end{tabular}




\begin{tabular}{|c|c|c|c|c|c|c|c|c|}
\hline Author, Year & $\begin{array}{c}\text { Key } \\
\text { Question }\end{array}$ & $\begin{array}{l}\text { 1. Was the } \\
\text { Selection of } \\
\text { Patients for } \\
\text { Inclusion } \\
\text { Unbiased? }\end{array}$ & $\begin{array}{l}\text { 2. Were the } \\
\text { Groups } \\
\text { Similar at } \\
\text { Baseline, or } \\
\text { Did the Design } \\
\text { or Analysis } \\
\text { Account for } \\
\text { Important } \\
\text { Confounding } \\
\text { and Modifying } \\
\text { Variables? }\end{array}$ & $\begin{array}{c}\text { 3. Were Outcome Assessors } \\
\text { Blinded, or Were Outcomes } \\
\text { Objectively Measured? }\end{array}$ & $\begin{array}{l}\text { 4. Were } \\
\text { Outcomes } \\
\text { Assessed } \\
\text { Using Valid } \\
\text { and Reliable } \\
\text { Measures? }\end{array}$ & \begin{tabular}{|c|} 
5. Were \\
Outcomes Pre- \\
specified and \\
Were Primaryl \\
Prespecified \\
Outcomes \\
Reported?
\end{tabular} & $\begin{array}{l}\text { 6. Was the } \\
\text { Study } \\
\text { (Protocol) } \\
\text { Registered? }\end{array}$ & Risk of Bias Quality Rating \\
\hline Cady, 2005 & 3 & Yes & Unclear & No & No & No & No & High \\
\hline Chan, 2020b & 4 & Yes & Unclear & Yes & Yes & Yes & No & Moderate \\
\hline Chiang, 2018 & $1,2,3$ & Yes & Yes & Yes & Yes & Yes & No & Low \\
\hline Cooper, 2001 & 2 & Unclear & No & No & Yes & Yes & No & High \\
\hline Cudnik, 2010 & 4 & Yes & Yes & Yes & Yes & Yes & No & Low \\
\hline Davis, 2005 & 3 & Yes & NR & Yes & No & Yes & No & Moderate \\
\hline Delorenzo, 2018 & 4 & Yes & No & Yes & Yes & Yes & No & Moderate \\
\hline Duckett, 2014 & 3 & Yes & NR & No & Unclear & Yes & No & High \\
\hline Eckstein, 2000 & 2 & Yes & Yes & Yes & Yes & Yes & No & Moderate \\
\hline Edwards, 2019 & 3 & Yes & Yes & No & Yes & Yes & No & Moderate \\
\hline Evans, 2016 & $1,2,3$ & Yes & Yes & Yes & Yes & Yes & No & Moderate \\
\hline Fouche, 2019 & 4 & Yes & Yes & Yes & Yes & Yes & No & Low \\
\hline Fukuda, 2020 & 3 & Yes & Yes & $\begin{array}{l}\text { Yes: mortality, ROSC } \\
\text { No: neurological function (CPC) }\end{array}$ & Yes & Yes & No & $\begin{array}{l}\text { Low: mortality, ROSC } \\
\text { Moderate: neurological function }\end{array}$ \\
\hline Gamberini, 2019 & 3 & Yes & Unclear & Yes & Yes & Yes & No & Moderate \\
\hline Garza, 2005 & 4 & Yes & No & No & Yes & Yes & No & High \\
\hline Gellerfors, 2014 & 4 & Yes & NR & No & No & Yes & No & High \\
\hline Hanif, 2010 & $1,2,3$ & Yes & Yes & Yes & Yes & Yes & No & Low \\
\hline Hansen, 2017 & $1,2,3$ & Yes & Yes & Yes & Yes & Yes & No & Low \\
\hline Hardy, 2018 & 1 & Yes & No & Yes & Yes & Yes & No & Moderate \\
\hline Hoffman, 2017 & 4 & Yes & Unclear & $\begin{array}{l}\text { Survival: Yes } \\
\text { Neurological function (GOS): } \\
\text { Unclear }\end{array}$ & Yes & Yes & No & Moderate \\
\hline Hossfeld, 2020 & 4 & Yes & Yes & No & Unclear & Yes & No & Moderate \\
\hline Jarvis, 2019 & 3,4 & Yes & Yes & No & Unclear & Yes & No & High \\
\hline Kang, 2016 & $1,2,3$ & Yes & Yes & $\begin{array}{l}\text { Yes for survival, no for CPC, } \\
\text { unclear for ROSC }\end{array}$ & Yes & Yes & No & $\begin{array}{l}\text { Low for survival, moderate for } \\
\text { CPC, moderate for ROSC }\end{array}$ \\
\hline Murray, 2000 & 4 & Yes & No & Yes & Yes & Yes & No & Moderate \\
\hline Nagao, 2012 & $1,2,3$ & Yes & No & Yes & Yes & Yes & No & Moderate \\
\hline Noda, 2007 & $1,2,3$ & Unclear & Unclear & Yes & Yes & Yes & No & High \\
\hline Nwanne, 2020 & 3,4 & Yes & Unclear & No & No & Yes & No & High \\
\hline $\begin{array}{l}\text { Ohashi-Fukuda, } \\
2017\end{array}$ & $1,2,3$ & Yes & Yes & Yes & Yes & Yes & No & Low \\
\hline
\end{tabular}




\begin{tabular}{|c|c|c|c|c|c|c|c|c|}
\hline Author, Year & $\begin{array}{c}\text { Key } \\
\text { Question }\end{array}$ & $\begin{array}{l}\text { 1. Was the } \\
\text { Selection of } \\
\text { Patients for } \\
\text { Inclusion } \\
\text { Unbiased? }\end{array}$ & \begin{tabular}{|} 
2. Were the \\
Groups \\
Similar at \\
Baseline, or \\
Did the Design \\
or Analysis \\
Account for \\
Important \\
Confounding \\
and Modifying \\
Variables?
\end{tabular} & $\begin{array}{c}\text { 3. Were Outcome Assessors } \\
\text { Blinded, or Were Outcomes } \\
\text { Objectively Measured? }\end{array}$ & $\begin{array}{c}\text { 4. Were } \\
\text { Outcomes } \\
\text { Assessed } \\
\text { Using Valid } \\
\text { and Reliable } \\
\text { Measures? }\end{array}$ & $\begin{array}{c}\text { 5. Were } \\
\text { Outcomes Pre- } \\
\text { specified and } \\
\text { Were Primary/ } \\
\text { Prespecified } \\
\text { Outcomes } \\
\text { Reported? }\end{array}$ & $\begin{array}{l}\text { 6. Was the } \\
\text { Study } \\
\text { (Protocol) } \\
\text { Registered? }\end{array}$ & Risk of Bias Quality Rating \\
\hline Olvera, 2018 & 4 & Yes & Unclear & Yes & Yes & Yes & No & High \\
\hline Powell, 2019 & 4 & Yes & Yes & Yes & Yes & Yes & No & Low \\
\hline Prekker, 2016 & 4 & Yes & Yes & No & Yes & Yes & No & Moderate \\
\hline Rocca 2000 & 4 & Yes & Unclear & No & Unclear & Yes & No & High \\
\hline Shin, 2012 & $1,2,3$ & No & Yes & Yes & Yes & Yes & No & Moderate \\
\hline Sobuwa, 2013 & 4 & Yes & Unclear & Unclear & Yes & Yes & No & Moderate \\
\hline Steuerwald, 2018 & 3 & Unclear & Unclear & Yes & Yes & Yes & No & Moderate \\
\hline Stockinger, 2004 & 2 & Yes & Yes & Yes & Yes & Yes & No & Low \\
\hline Tanabe, 2013 & 3,4 & Yes & Yes & Yes & Yes & Yes & No & Low \\
\hline Vilke, 1994 & 4 & Yes & Unclear & Yes & Yes & No & No & Moderate \\
\hline Wang, 2006 & 4 & No & Unclear & No & Yes & Yes & No & High \\
\hline Wang, 2012 & 3 & Yes & Unclear & Yes & Yes & Yes & No & Low \\
\hline Yanagawa, 2010 & $1,2,3$ & Yes & Yes & Yes for PROSC, no for CPC & Yes & Yes & No & Moderate \\
\hline Yuksen, 2020 & 2 & Yes & No & Yes & Yes & Yes & No & High \\
\hline
\end{tabular}

$\mathrm{CPC}=$ cerebral performance category; $\mathrm{NR}=$ not reported; $\mathrm{PROSC}=$ prehospital return of spontaneous circulation; ROSC $=$ return of spontaneous circulation 


\section{Appendix H. Meta-Analysis: Primary Analyses}

Figure H-1. BVM versus SGA (KQ1) pooled estimate of survival by emergency type, age, and study design

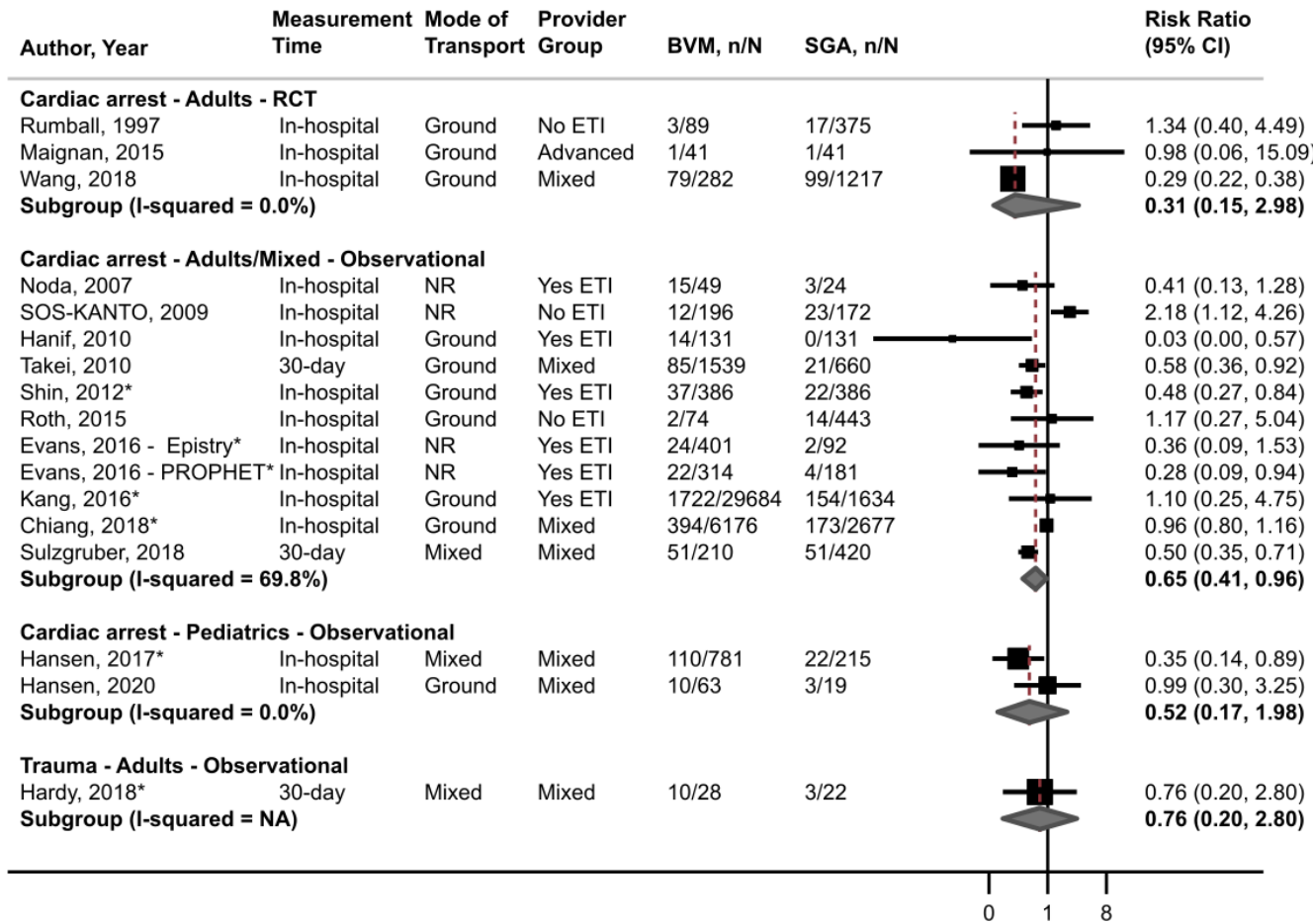

Favors BVM Favors SGA

$\mathrm{BVM}=$ bag valve mask; $\mathrm{CI}=$ confidence interval; $\mathrm{ETI}=$ endotracheal intubation; $\mathrm{KQ}=$ Key Question; NA = not applicable; NR $=$ not reported; $\mathrm{RCT}=$ randomized control trial; $\mathrm{SGA}=$ supraglottic airway

${ }^{a}$ Asterisk indicates where adjusted results were used in the analysis 
Figure H-2. BVM versus SGA (KQ1) pooled estimate of neurological function by emergency type, age, and study design

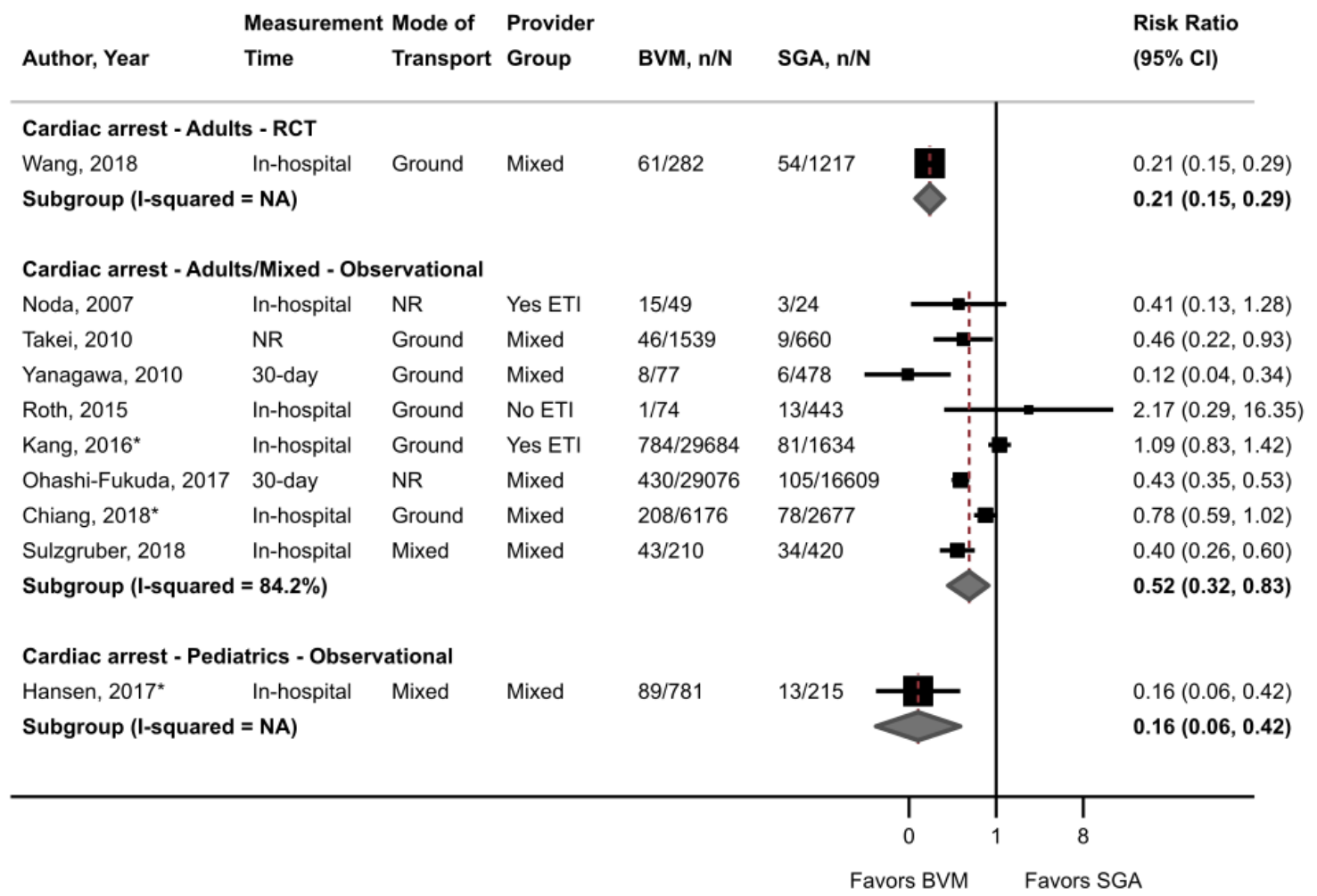

$\mathrm{BVM}=$ bag valve mask; $\mathrm{CI}=$ confidence interval; $\mathrm{ETI}=$ endotracheal intubation; $\mathrm{KQ}=$ Key Question; $\mathrm{NA}=$ not applicable; NR $=$ not reported; $\mathrm{RCT}=$ randomized control trial; $\mathrm{SGA}=$ supraglottic airway

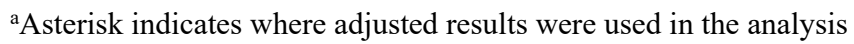


Figure H-3. BVM versus SGA (KQ1) pooled estimate of ROSC by emergency type, age, and study design

\begin{tabular}{|c|c|c|c|c|c|c|}
\hline Author, Year & $\begin{array}{l}\text { Measurement } \\
\text { Time }\end{array}$ & $\begin{array}{l}\text { Mode of } \\
\text { Transport }\end{array}$ & $\begin{array}{l}\text { Provider } \\
\text { Group }\end{array}$ & $\mathrm{BVM}, \mathrm{n} / \mathrm{N}$ & SGA, n/N & $\begin{array}{l}\text { Risk Ratio } \\
(95 \% \mathrm{Cl})\end{array}$ \\
\hline \multicolumn{7}{|c|}{ Cardiac arrest - Adults - RCT } \\
\hline Maignan, 2015 & Prehospital & Ground & Advanced & $9 / 41$ & $7 / 41$ & $0.78(0.32,1.89)$ \\
\hline Fiala, 2017 & Not specified & Ground & No ETI & $7 / 41$ & $9 / 35$ & $1.51(0.63,3.63)$ \\
\hline Wang, 2018 & In-hospital & Ground & Mixed & $107 / 282$ & $432 / 1217$ & $0.94(0.79,1.11)$ \\
\hline \multicolumn{6}{|c|}{ Subgroup $(\mathrm{I}-\mathrm{squared}=0.0 \%)$} & $0.94(0.76,1.27)$ \\
\hline \multicolumn{7}{|c|}{ Cardiac arrest - Adults - Observational } \\
\hline SOS-KANTO, 2009 & ED & NR & No ETI & $80 / 200$ & $67 / 173$ & $0.97(0.75,1.25)$ \\
\hline Takei, 2010 & Sustained & Ground & Mixed & $327 / 1539$ & $133 / 660$ & $0.95(0.79,1.14)$ \\
\hline Yanagawa, 2010 & Prehospital & Ground & Mixed & $13 / 77$ & $37 / 478-$ & $0.46(0.26,0.82)$ \\
\hline Chien, 2012 & Not specified & Ground & No ETI & $32 / 89$ & $147 / 309$ & $1.32(0.98,1.79)$ \\
\hline Nagao, 2012 & Overall & Ground & Yes ETI & $16 / 156$ & $36 / 189$ & $1.86(1.07,3.22)$ \\
\hline Roth, $2015^{*}$ & Sustained & Ground & No ETI & $5 / 74$ & $61 / 443$ & $1.22(0.50,3.00)$ \\
\hline Kang, 2016 & Prehospital & Ground & Yes ETI & $920 / 29684$ & $87 / 1634$ & $1.72(1.39,2.13)$ \\
\hline Chiang, 2018* & Sustained & Ground & Mixed & $1327 / 6176$ & $649 / 2677$ & $1.12(1.03,1.22)$ \\
\hline Sulzgruber, 2018 & Sustained & Mixed & Mixed & $77 / 210$ & $113 / 420$ & $0.73(0.58,0.93)$ \\
\hline \multicolumn{6}{|c|}{ Subgroup (I-squared = 87.2\%) } & $1.07(0.82,1.40)$ \\
\hline \multicolumn{7}{|c|}{ Cardiac arrest - Pediatrics - Observational } \\
\hline Hansen, $2017^{\star}$ & Sustained & Mixed & Mixed & $141 / 781$ & $58 / 215$ & $1.19(0.75,1.88)$ \\
\hline \multicolumn{6}{|c|}{ Subgroup (I-squared = NA) } & $1.19(0.75,1.88)$ \\
\hline & & & & & $T$ & \\
\hline & & & & & Favors B & SGA \\
\hline
\end{tabular}

$\mathrm{BVM}=$ bag valve mask; $\mathrm{CI}=$ confidence interval; $\mathrm{ED}=$ emergency department; $\mathrm{ETI}=$ endotracheal intubation; $\mathrm{KQ}=\mathrm{Key}$ Question; NA = not applicable; NR = not reported; RCT = randomized control trial; ROSC = return of spontaneous circulation; SGA $=$ supraglottic airway

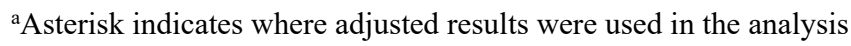


Figure H-4. BVM versus ETI (KQ2) pooled estimate of survival by emergency type, age, and study design

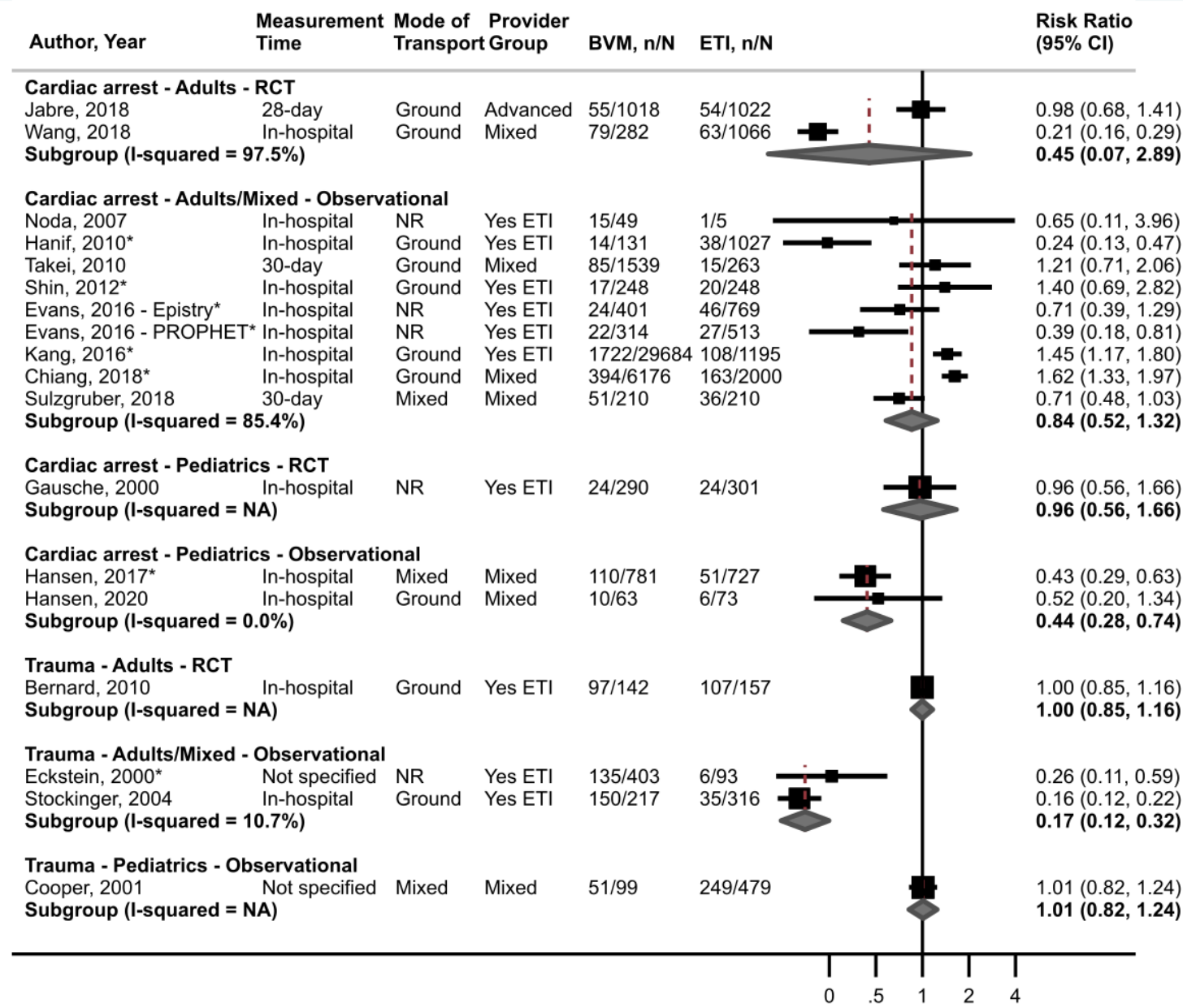

Favors BVM $\quad$ Favors ETI

$\mathrm{BVM}=$ bag valve mask; $\mathrm{CI}=$ confidence interval; $\mathrm{ETI}=$ endotracheal intubation; $\mathrm{KQ}=$ Key Question; $\mathrm{NA}=$ not applicable; NR $=$ not reported; $\mathrm{RCT}=$ randomized control trial

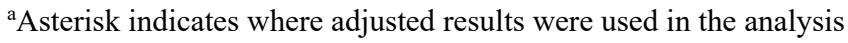


Figure H-5. BVM versus ETI (KQ2) pooled estimate of neurological function by emergency type, age, and study design

\begin{tabular}{|c|c|c|c|c|c|c|}
\hline Author, Year & $\begin{array}{l}\text { Measurement } \\
\text { Time }\end{array}$ & $\begin{array}{l}\text { Mode of } \\
\text { Transport }\end{array}$ & $\begin{array}{l}\text { Provider } \\
\text { t Group }\end{array}$ & $\mathrm{BVM}, \mathrm{n} / \mathrm{N}$ & $\mathrm{ETI}, \mathrm{n} / \mathrm{N}$ & $\begin{array}{l}\text { Risk Ratio } \\
(95 \% \mathrm{CI})\end{array}$ \\
\hline \multicolumn{7}{|c|}{ Cardiac arrest - Adults - RCT } \\
\hline Jabre, 2018 & 28-day & Ground & Advanced & $44 / 1018$ & $43 / 1022$ & $0.97(0.65,1.47)$ \\
\hline \multicolumn{6}{|c|}{ Subgroup (I-squared = NA) } & $0.97(0.65,1.47)$ \\
\hline \multicolumn{7}{|c|}{ Cardiac arrest - Adults- Observational } \\
\hline Takei, 2010 & Not specified & Ground & Mixed & $46 / 1539$ & $4 / 263$ & $0.51(0.18,1.40)$ \\
\hline Yanagawa, 2010 & 30-day & Ground & Mixed & $8 / 77$ & $2 / 158$ & $0.12(0.03,0.56)$ \\
\hline Kang, 2016* & In-hospital & Ground & Yes ETI & $784 / 29684$ & $53 / 1195$ & $1.39(1.00,1.93)$ \\
\hline Ohashi-Fukuda, $2017^{\star}$ & 30-day & NR & Mixed & $430 / 29076$ & $26 / 3849$ & $0.47(0.31,0.72)$ \\
\hline Chiang, 2018* & In-hospital & Ground & Mixed & $208 / 6176$ & $64 / 2000$ & $1.20(0.88,1.63)$ \\
\hline Sulzgruber, 2018 & In-hospital & Mixed & Mixed & $43 / 210$ & $19 / 210$ & $0.44(0.27,0.73)$ \\
\hline \multicolumn{6}{|c|}{ Subgroup (l-squared = 84.1\%) } & $0.65(0.32,1.13)$ \\
\hline \multicolumn{7}{|c|}{ Cardiac arrest - Pediatrics - RCT } \\
\hline Gausche, 2000 & In-hospital & NR & Yes ETI & $10 / 290$ & $15 / 301$ & $1.45(0.66,3.16)$ \\
\hline \multicolumn{6}{|c|}{ Subgroup (I-squared = NA) } & $1.45(0.66,3.16)$ \\
\hline \multicolumn{7}{|c|}{ Cardiac arrest - Pediatrics - Observational } \\
\hline Hansen, 2017* & In-hospital & Mixed & Mixed & $89 / 781$ & $34 / 727$ & $0.33(0.20,0.53)$ \\
\hline \multicolumn{6}{|c|}{ Subgroup $(\mathrm{I}-\mathrm{squared}=\mathrm{NA})$} & $0.33(0.20,0.53)$ \\
\hline
\end{tabular}

Favors BVM Favors ETI

$\mathrm{BVM}=$ bag valve mask; $\mathrm{CI}$ = confidence interval; $\mathrm{ETI}=$ endotracheal intubation; $\mathrm{KQ}=$ Key Question; NA = not applicable; NR $=$ not reported; $\mathrm{RCT}=$ randomized control trial

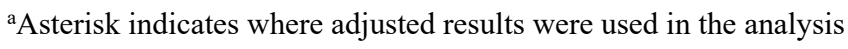


Figure H-6. BVM versus ETI (KQ2) pooled estimate of ROSC by emergency type, age, and study design

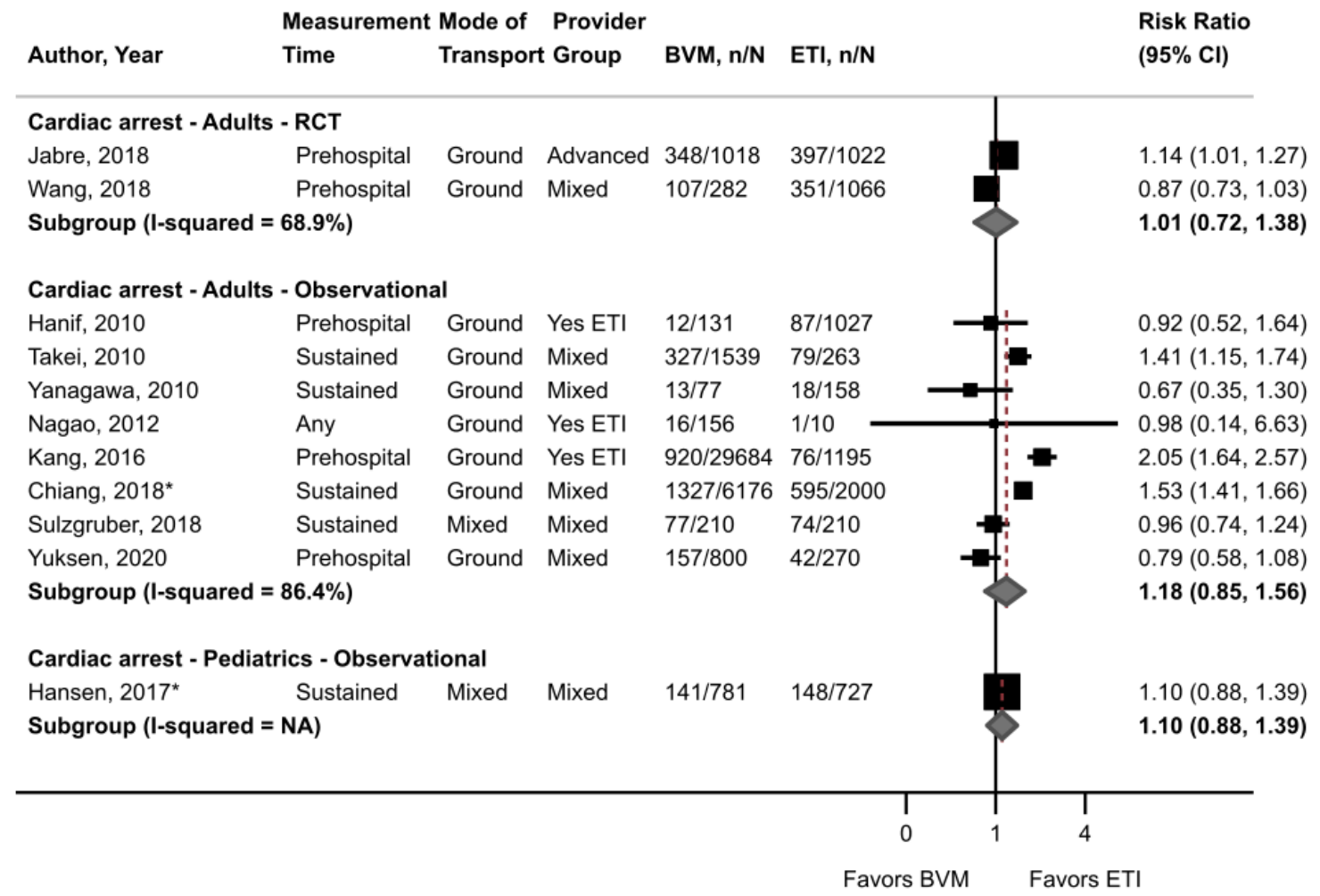

$\mathrm{BVM}=$ bag valve mask; $\mathrm{CI}=$ confidence interval; $\mathrm{ETI}=$ endotracheal intubation; $\mathrm{KQ}=$ Key Question; NA = not applicable; $\mathrm{RCT}=$ randomized control trial; ROSC $=$ return of spontaneous circulation

${ }^{a}$ Asterisk indicates where adjusted results were used in the analysis 
Figure H-7. SGA versus ETI (KQ3) pooled estimate of survival by emergency type, age, and study design

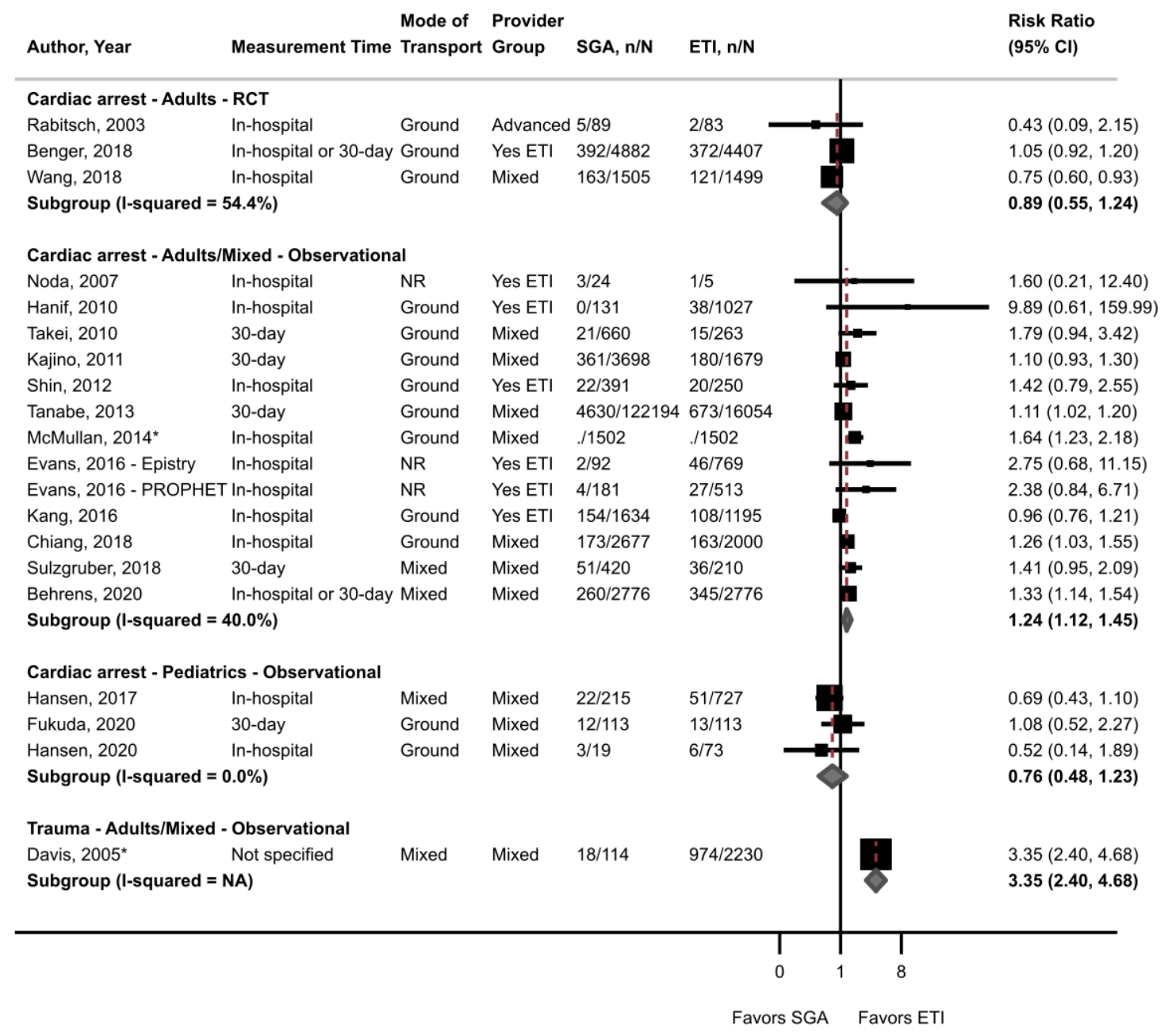

$\mathrm{CI}=$ confidence interval; $\mathrm{ETI}=$ endotracheal intubation; $\mathrm{KQ}=$ Key Question; $\mathrm{NA}=$ not applicable; $\mathrm{NR}=$ not reported; $\mathrm{RCT}=$ randomized control trial; SGA = supraglottic airway

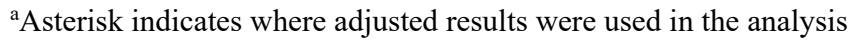


Figure H-8. SGA versus ETI (KQ3) pooled estimate of neurological function by emergency type,

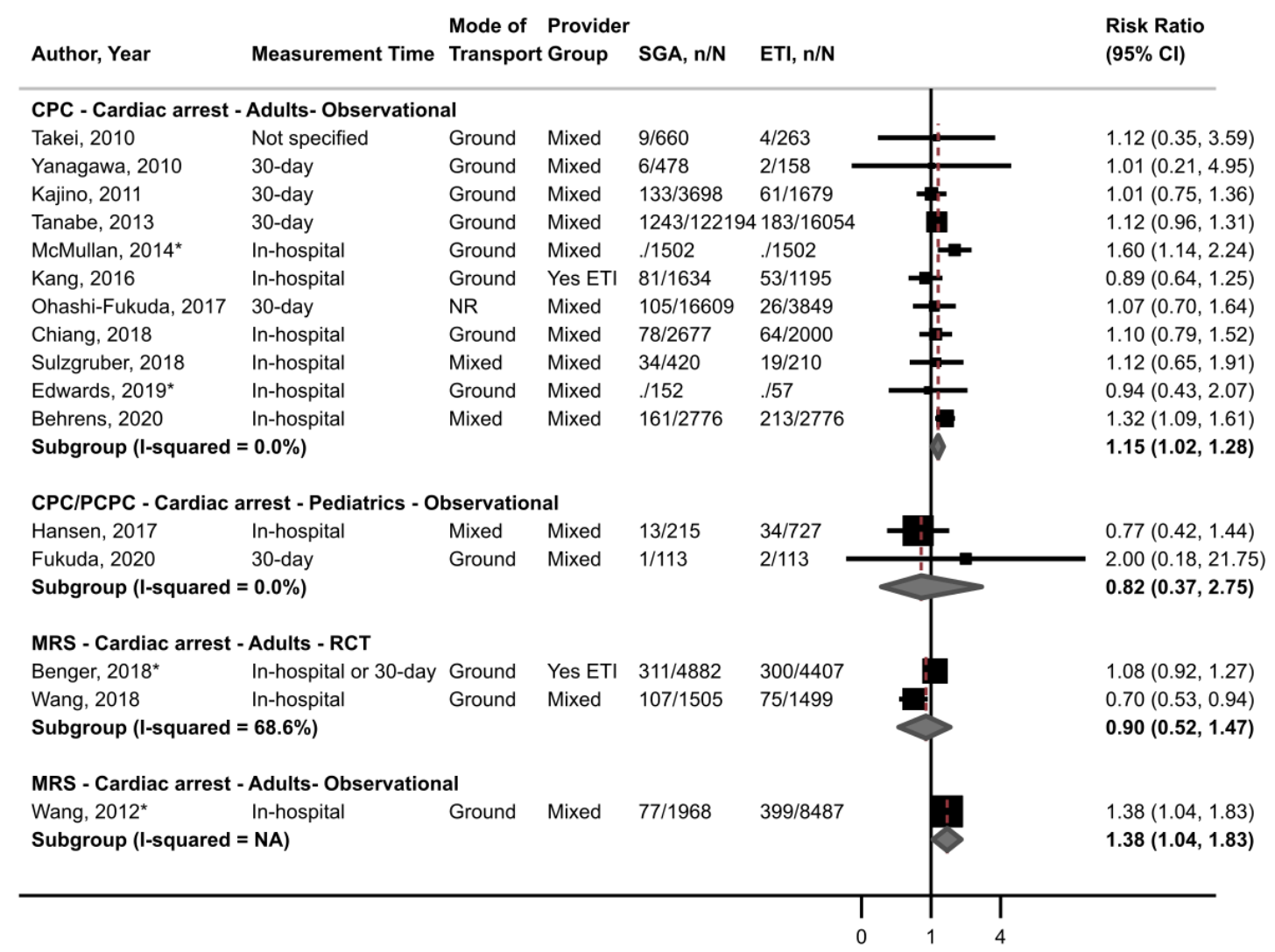

Favors SGA Favors ETI

$\mathrm{CI}=$ confidence interval; $\mathrm{CPC}=$ Cerebral Performance Category; $\mathrm{ETI}=$ endotracheal intubation; $\mathrm{KQ}=$ Key Question; $\mathrm{MRS}=$ Modified Rankin Scale; NA = not applicable; NR = not reported; PCPC = Pediatric Cerebral Performance Category; RCT = randomized control trial; SGA = supraglottic airway

${ }^{\mathrm{a}}$ Asterisk indicates where adjusted results were used in the analysis 
Figure H-9. SGA versus ETI (KQ3) pooled estimate of ROSC by emergency type, age, and study design

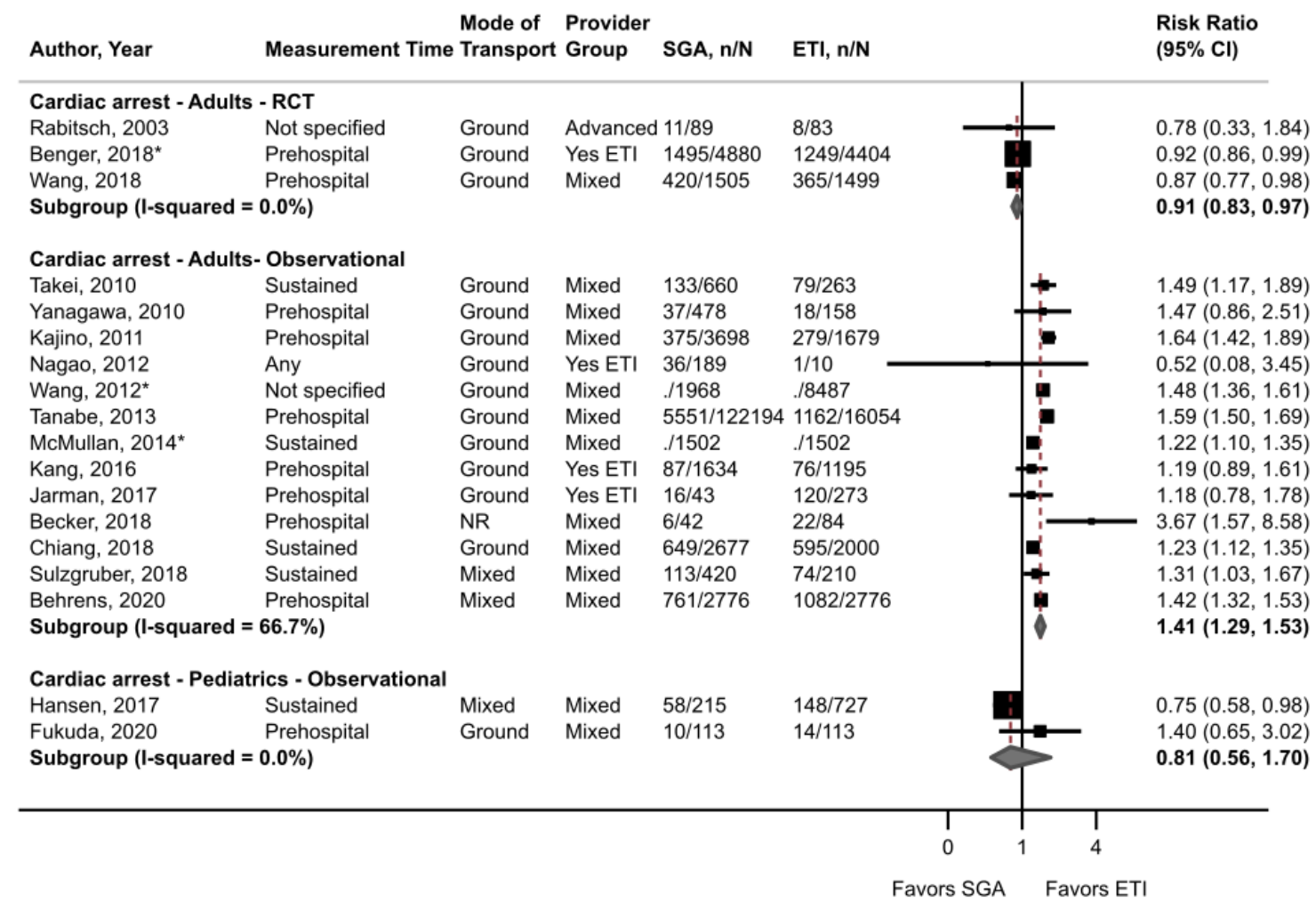

$\mathrm{CI}=$ confidence interval; $\mathrm{CPC}=$ Cerebral Performance Category; $\mathrm{ETI}=$ endotracheal intubation; $\mathrm{KQ}=\mathrm{Key}$ Question; $\mathrm{NR}=$ not reported; RCT = randomized control trial; ROSC = return of spontaneous circulation; $\mathrm{SGA}=$ supraglottic airway

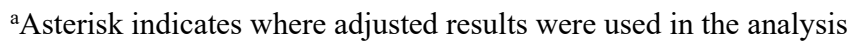


Figure H-10. SGA versus ETI (KQ3) pooled estimate of first-pass success by emergency type, age,

\begin{tabular}{|c|c|c|c|c|}
\hline $\begin{array}{l}\text { Mode of } \\
\text { Transport }\end{array}$ & $\begin{array}{l}\text { Provider } \\
\text { Group }\end{array}$ & SGA, n/N & ETI, n/N & $\begin{array}{l}\text { Risk Ratio } \\
(95 \% \mathrm{Cl})\end{array}$ \\
\hline $\begin{array}{l}\text { Cardiac arrest - Adults - RCT } \\
\text { Wang, } 2018 \quad \text { Ground } \\
\text { Subgroup (I-squared = NA) }\end{array}$ & Mixed & $1359 / 1505$ & $773 / 1499$ & $\begin{array}{l}0.57(0.54,0.60) \\
\mathbf{0 . 5 7}(\mathbf{0 . 5 4}, \mathbf{0 . 6 0})\end{array}$ \\
\hline $\begin{array}{lc}\text { Cardiac arrest }- \text { Adults - Obse } \\
\text { Hankins, 1993 } & \text { Ground } \\
\text { Gahan, 2011* } & \text { Ground } \\
\text { Jarman, 2017 } & \text { Ground } \\
\text { Jarvis, 2019 } & \text { NR } \\
\text { Subgroup (I-squared }=\mathbf{0 . 0 \% )}\end{array}$ & $\begin{array}{l}\text { rvational } \\
\text { Mixed } \\
\text { Mixed } \\
\text { Yes ETI } \\
\text { Mixed }\end{array}$ & $\begin{array}{l}180 / 240 \\
137 / 156 \\
33 / 43 \\
5144 / 5671\end{array}$ & $\begin{array}{l}180 / 269 \\
83 / 144 \\
184 / 273 \\
9942 / 13735\end{array}$ & $\begin{array}{l}0.89(0.80,1.00) \\
0.67(0.52,0.87) \\
0.88(0.73,1.06) \\
0.80(0.79,0.81) \\
\mathbf{0 . 8 0}(\mathbf{0 . 7 8}, \mathbf{0 . 8 6})\end{array}$ \\
\hline $\begin{array}{lc}\text { Cardiac arrest } & \text { Pediatrics - Ol } \\
\text { Jarvis, } 2019 & \text { NR } \\
\text { Hansen, } 2020 & \text { Ground } \\
\text { Subgroup (I-squared }=\mathbf{0 . 0 \% )}\end{array}$ & $\begin{array}{l}\text { bservation } \\
\text { Mixed } \\
\text { Mixed }\end{array}$ & $\begin{array}{l}\text { al } \\
34 / 40 \\
18 / 19\end{array}$ & $\begin{array}{l}184 / 313 \\
43 / 73\end{array}$ & $\begin{array}{l}0.69(0.59,0.81) \\
0.62(0.50,0.77) \\
\mathbf{0 . 6 7}(\mathbf{0 . 5 6}, \mathbf{0 . 7 8})\end{array}$ \\
\hline $\begin{array}{l}\text { Trauma - Adults - Observation } \\
\text { Jarvis, } 2019 \quad \text { NR } \\
\text { Subgroup (I-squared = NA) }\end{array}$ & $\stackrel{\text { Ial }}{\text { Mixed }}$ & $272 / 306$ & $1295 / 1837$ & $\begin{array}{l}0.79(0.75,0.83) \\
\mathbf{0 . 7 9}(\mathbf{0 . 7 5}, \mathbf{0 . 8 3})\end{array}$ \\
\hline $\begin{array}{l}\text { Trauma - Pediatrics - Observa } \\
\text { Jarvis, } 2019 \quad \text { NR } \\
\text { Subgroup (I-squared = NA) }\end{array}$ & $\begin{array}{l}\text { Itional } \\
\text { Mixed }\end{array}$ & $7 / 8$ & $36 / 61$ & $\begin{array}{l}0.67(0.48,0.94) \\
\mathbf{0 . 6 7}(\mathbf{0 . 4 8 , 0 . 9 4 )}\end{array}$ \\
\hline $\begin{array}{l}\text { Medical - Adults - RCT } \\
\text { Frascone, } 2011 \quad \text { Ground } \\
\text { Subgroup (I-squared = NA) }\end{array}$ & Yes ETI & $87 / 128$ & $51 / 76$ & $\begin{array}{l}0.99(0.81,1.20) \\
\mathbf{0 . 9 9}(\mathbf{0 . 8 1}, \mathbf{1 . 2 0})\end{array}$ \\
\hline $\begin{array}{l}\text { Medical - Adults - Observatio } \\
\text { Jarvis, } 2019 \quad \mathrm{NR} \\
\text { Subgroup (I-squared = NA) }\end{array}$ & $\begin{array}{l}\text { nal } \\
\text { Mixed }\end{array}$ & $690 / 820$ & $4787 / 6477$ & $\begin{array}{l}0.88(0.85,0.91) \\
\mathbf{0 . 8 8}(\mathbf{0 . 8 5}, \mathbf{0 . 9 1})\end{array}$ \\
\hline $\begin{array}{l}\text { Medical - Pediatrics - Observ } \\
\text { Jarvis, } 2019 \quad \text { NR } \\
\text { Subgroup (I-squared = NA) }\end{array}$ & $\begin{array}{l}\text { ational } \\
\text { Mixed }\end{array}$ & $3 / 4$ & $55 / 96$ & $\begin{array}{l}0.76(0.42,1.38) \\
\mathbf{0 . 7 6}(\mathbf{0 . 4 2 ,} \mathbf{1 . 3 8})\end{array}$ \\
\hline $\begin{array}{l}\text { Mixed - Adults - Observationa } \\
\text { McCall, } 2008 \quad \text { Ground } \\
\text { Steuerwald, } 2018 \quad \text { Mixed } \\
\text { Subgroup (I-squared }=\mathbf{0 . 0 \%}\end{array}$ & $\begin{array}{l}\text { Yes ETI } \\
\text { Mixed }\end{array}$ & $\begin{array}{l}42 / 52 \\
99 / 104\end{array}$ & $\begin{array}{l}26 / 46 \\
107 / 205\end{array}$ & $\begin{array}{l}0.70(0.53,0.93) \\
0.55(0.48,0.63) \\
\mathbf{0 . 5 7}(\mathbf{0 . 4 7}, \mathbf{0 . 7 9})\end{array}$ \\
\hline & & & & \\
\hline
\end{tabular}

Favors SGA Favors ETI

$\mathrm{CI}=$ confidence interval; ETI $=$ endotracheal intubation; $\mathrm{KQ}=$ Key Question; $\mathrm{NA}=$ not applicable; $\mathrm{NR}=$ not reported; $\mathrm{RCT}=$ randomized control trial; SGA = supraglottic airway

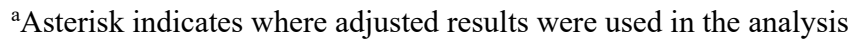


Figure H-11. SGA versus ETI (KQ3) pooled estimate of overall success by emergency type, age, and study design

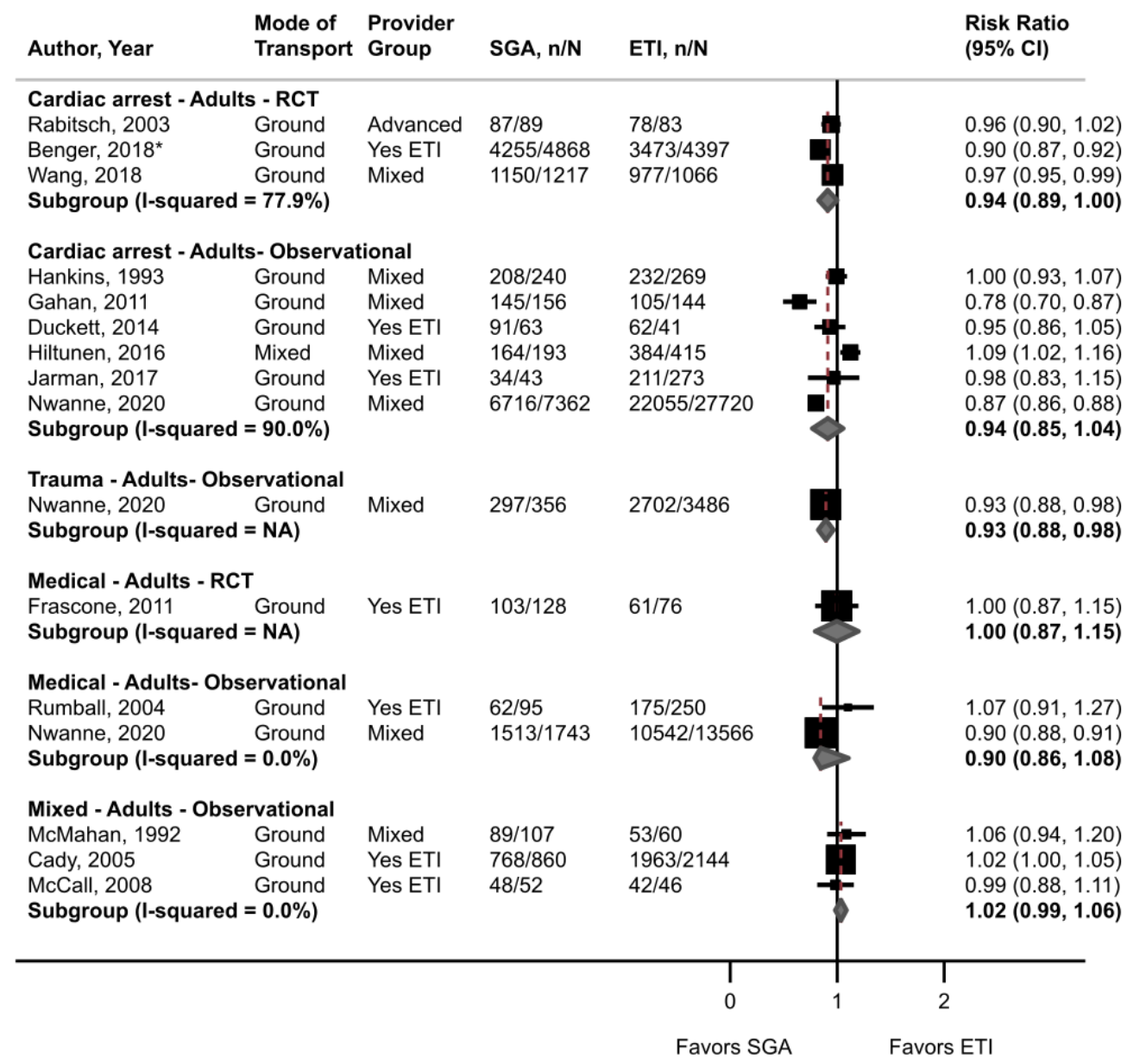

$\mathrm{CI}=$ confidence interval; $\mathrm{ETI}=$ endotracheal intubation; $\mathrm{KQ}=$ Key Question; $\mathrm{NA}=$ not applicable; $\mathrm{RCT}=$ randomized control trial; SGA = supraglottic airway

${ }^{a}$ Asterisk indicates where adjusted results were used in the analysis 
Figure H-12. RSI versus no medication (KQ4) pooled estimate of survival by emergency type, age, and study design

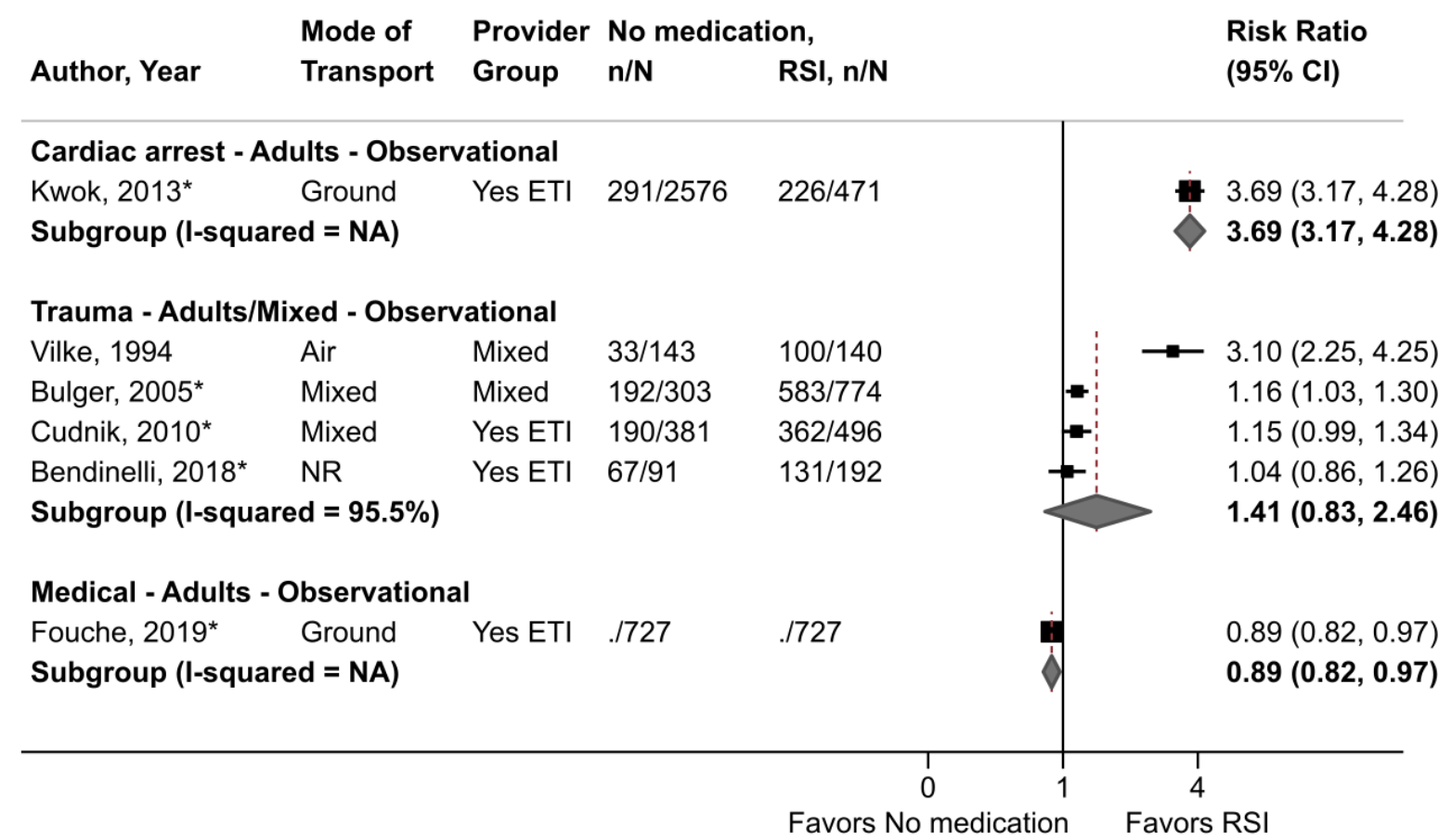

$\mathrm{CI}=$ confidence interval; $\mathrm{ETI}=$ endotracheal intubation; $\mathrm{KQ}=$ Key Question; $\mathrm{NA}=$ not applicable; $\mathrm{NR}=$ not reported; $\mathrm{RCT}=$ randomized control trial; RSI = rapid sequence intubation

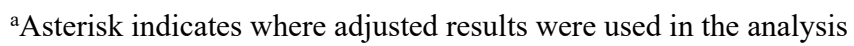


Figure H-13. RSI versus no medication (KQ4) pooled estimate of success by emergency type, age, and study design

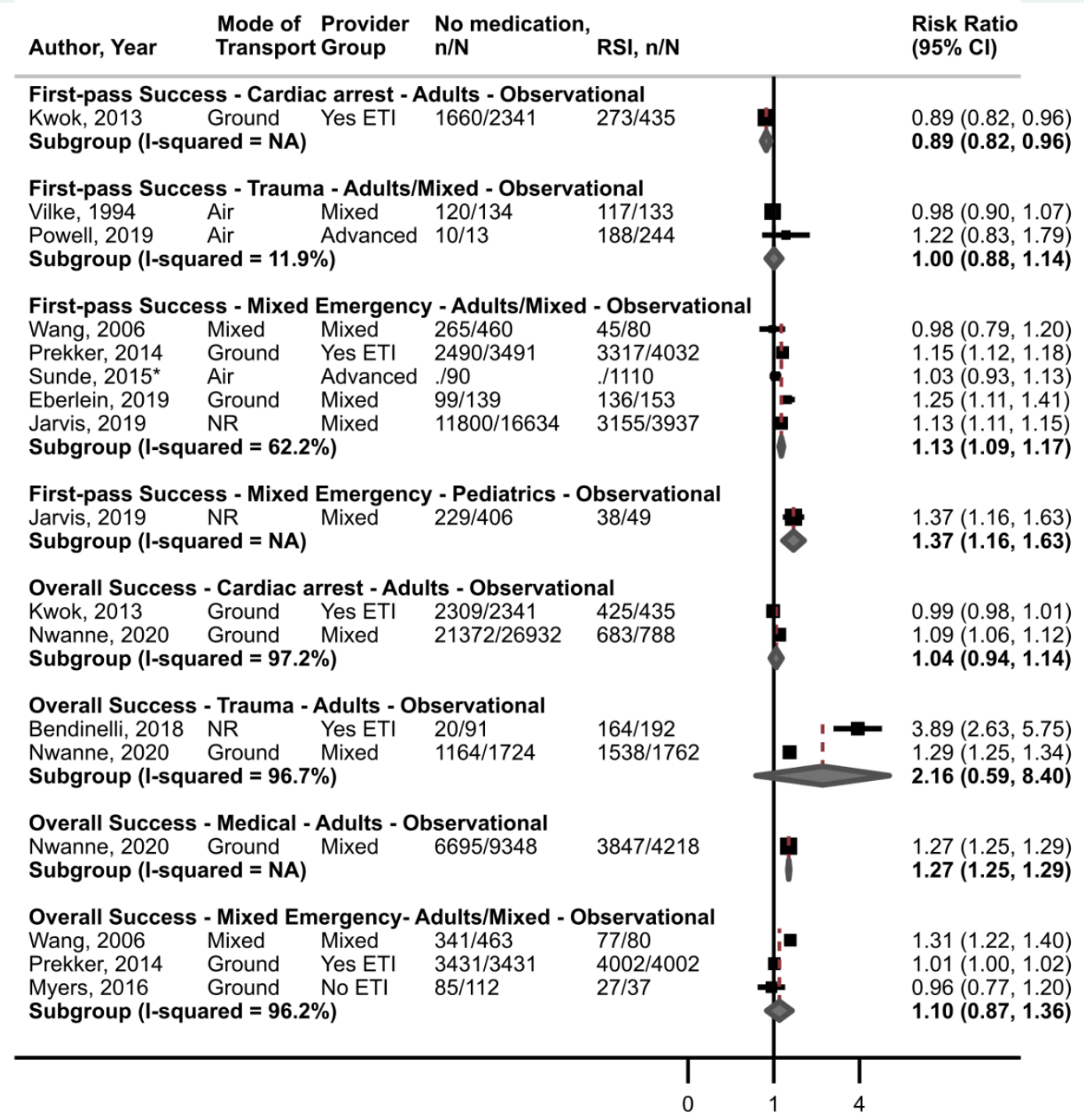

Favors No medication Favors RSI

$\mathrm{CI}=$ confidence interval; ETI $=$ endotracheal intubation; $\mathrm{KQ}=$ Key Question; $\mathrm{NA}=$ not applicable; $\mathrm{NR}=$ not reported; RSI = rapid sequence intubation

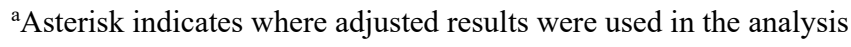


Figure H-14. RSI versus sedation-facilitated (KQ4) pooled estimate of first-pass success by emergency type, age, and study design

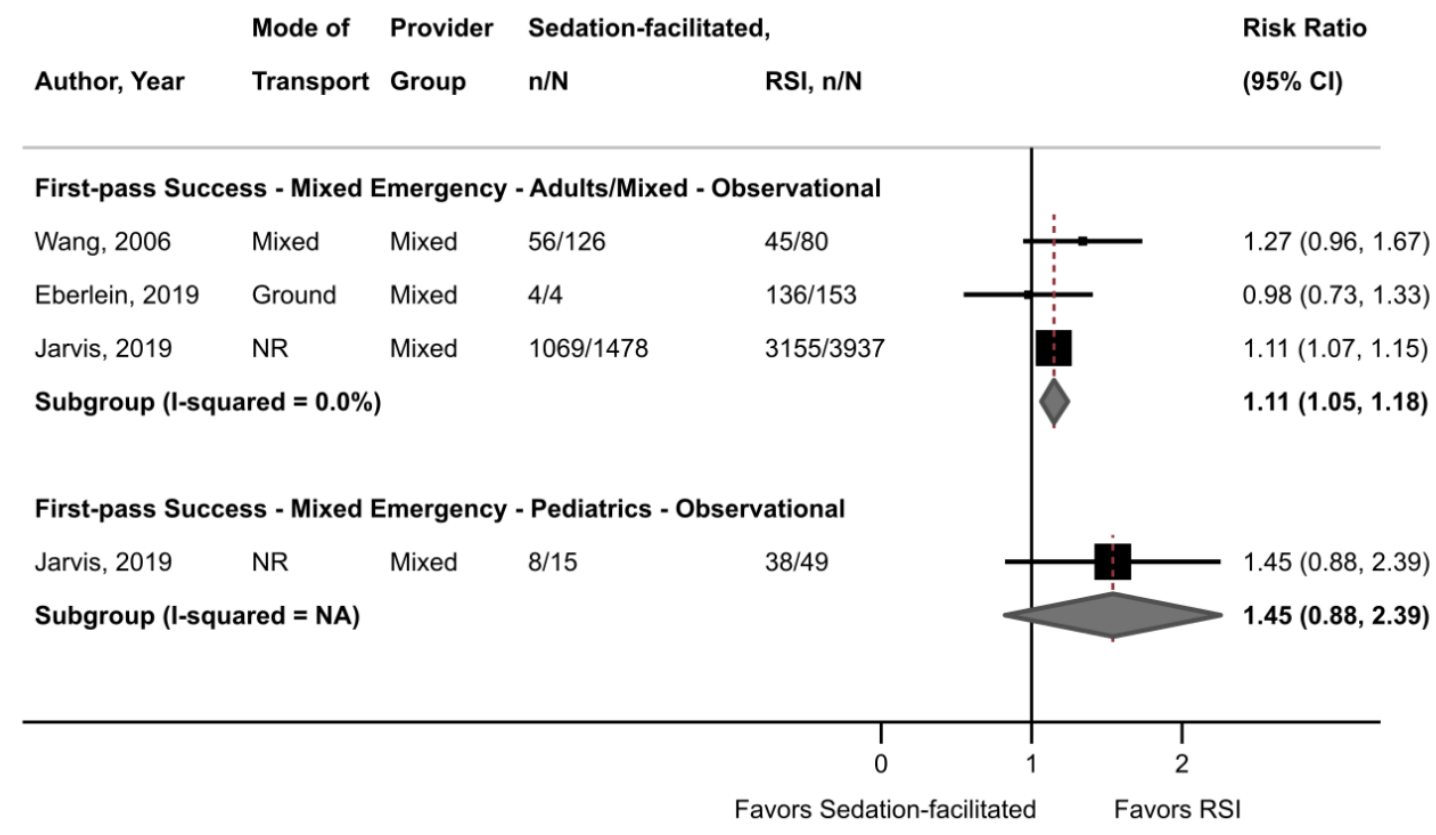

$\mathrm{CI}=$ confidence interval; KQ = Key Question; NA = not applicable; NR = not reported; RSI = rapid sequence intubation

Figure H-15. Sedation-facilitated versus no medication (KQ4) pooled estimate of first-pass success by emergency type, age, and study design

\begin{tabular}{|c|c|c|c|c|c|}
\hline Author, Year & $\begin{array}{l}\text { Mode of } \\
\text { Transport }\end{array}$ & $\begin{array}{l}\text { Provider } \\
\text { t Group }\end{array}$ & $\begin{array}{l}\text { No medication, } \\
n / N\end{array}$ & $\begin{array}{l}\text { Sedation-facilitated, } \\
n / N\end{array}$ & $\begin{array}{l}\text { Risk Ratio } \\
(95 \% \mathrm{Cl})\end{array}$ \\
\hline \multicolumn{6}{|c|}{ First-pass Success - Mixed Emergency - Adults/Mixed - Observational } \\
\hline Wang, 2006 & Mixed & Mixed & $265 / 460$ & $56 / 126$ & $0.77(0.63,0.95)$ \\
\hline Eberlein, 2019 & Ground & Mixed & $99 / 139$ & $4 / 4$ & $1.27(0.93,1.73)$ \\
\hline Jarvis, 2019 & NR & Mixed & $11800 / 16634$ & $1069 / 1478$ & $1.02(0.99,1.05)$ \\
\hline \multicolumn{5}{|c|}{ Subgroup $(\mathrm{I}$-squared $=0.0 \%)$} & $1.02(0.77,1.27)$ \\
\hline \multicolumn{6}{|c|}{ First-pass Success - Mixed Emergency - Pediatrics - Observational } \\
\hline Jarvis, 2019 & NR & Mixed & $229 / 406$ & $8 / 15$ & $0.95(0.58,1.53)$ \\
\hline \multicolumn{5}{|c|}{ Subgroup (I-squared = NA) } & $0.95(0.58,1.53)$ \\
\hline
\end{tabular}

$\mathrm{CI}=$ confidence interval; KQ = Key Question; NA = not applicable; NR = not reported 
Figure H-16. Direct versus video (KQ4) pooled estimate of success by emergency type, age, and study design

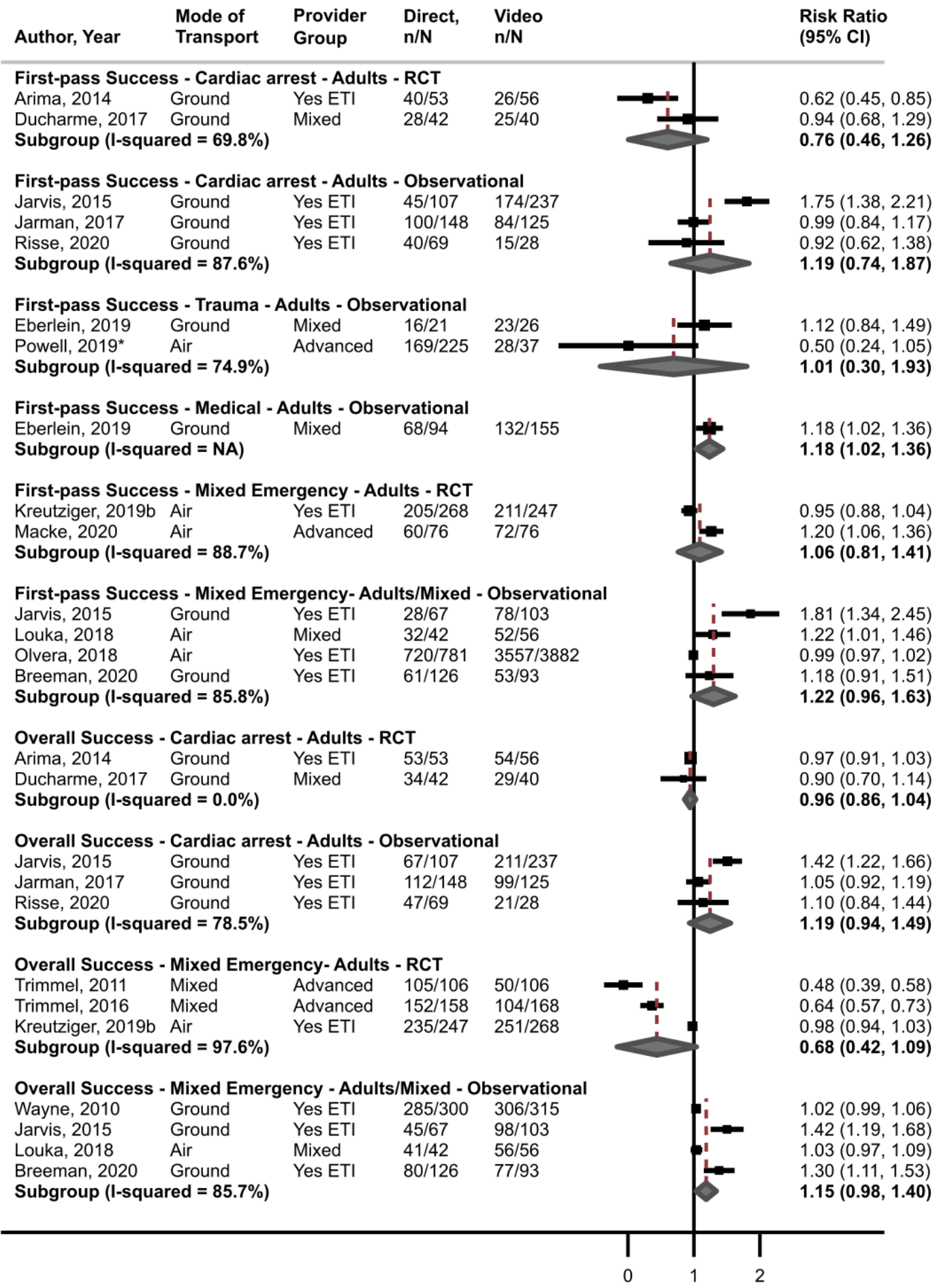

Favors Direct Favors Video

$\mathrm{CI}=$ confidence interval; $\mathrm{ETI}=$ endotracheal intubation; $\mathrm{KQ}=$ Key Question; $\mathrm{NA}=$ not applicable; $\mathrm{RCT}=$ randomized control trial

${ }^{\mathrm{a} A s t e r i s k}$ indicates where adjusted results were used in the analysis 
Figure H-17. Cardiac arrest versus medical (KQ4) pooled estimate of success

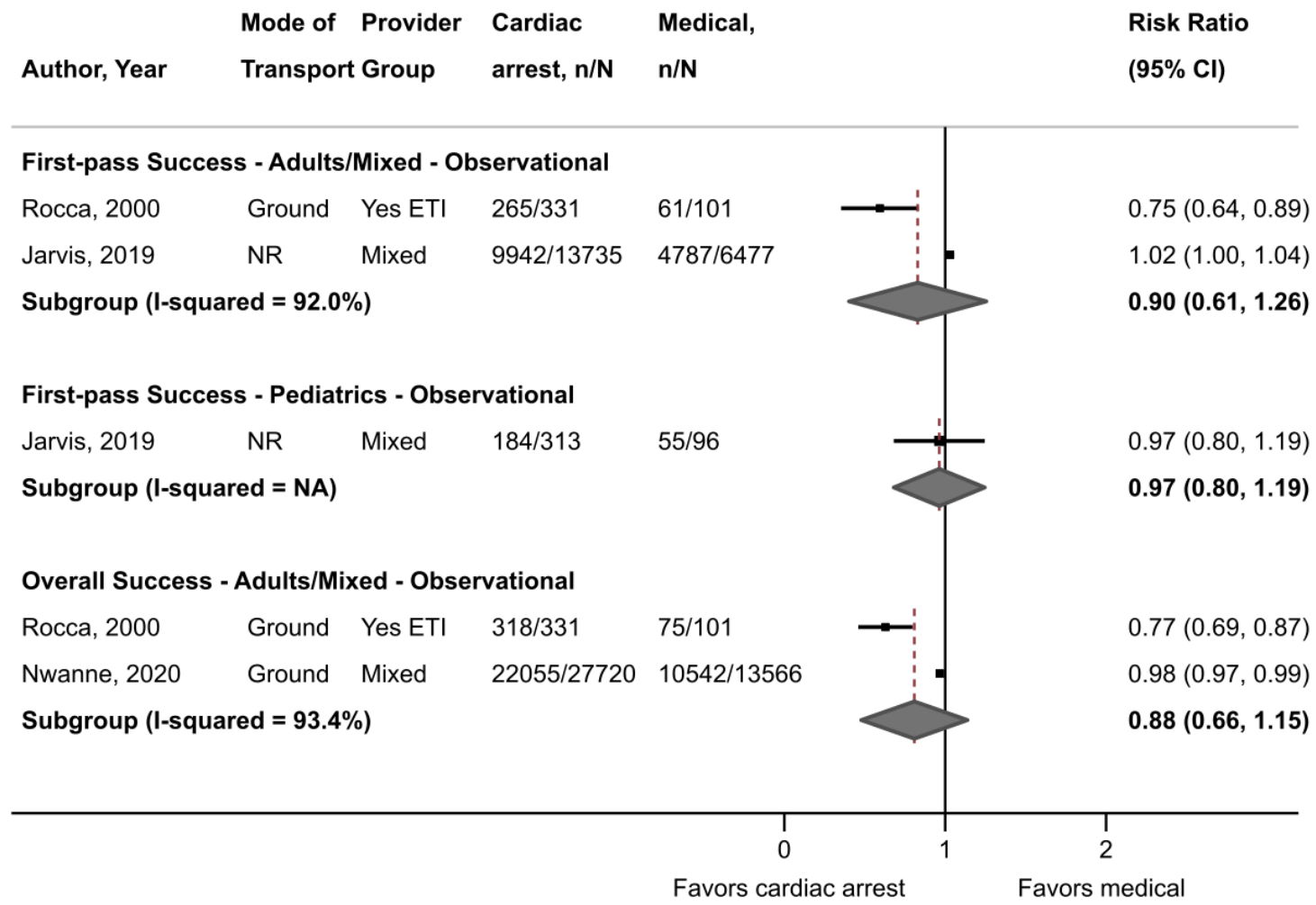

$\mathrm{CI}=$ confidence interval; $\mathrm{ETI}=$ endotracheal intubation; $\mathrm{KQ}=$ Key Question; $\mathrm{NA}=$ not applicable; $\mathrm{NR}=$ not reported 
Figure H-18. Cardiac arrest versus trauma (KQ4) pooled estimate of success by age and study design

\begin{tabular}{|c|c|c|c|c|c|}
\hline Author, Year & $\begin{array}{l}\text { Mode of } \\
\text { Transport }\end{array}$ & $\begin{array}{l}\text { Provider } \\
\text { Group }\end{array}$ & $\begin{array}{l}\text { Cardiac } \\
\text { arrest, n/N }\end{array}$ & $\begin{array}{l}\text { Trauma, } \\
\mathrm{n} / \mathrm{N}\end{array}$ & $\begin{array}{l}\text { Risk Ratio } \\
(95 \% \mathrm{Cl})\end{array}$ \\
\hline \multicolumn{6}{|c|}{ First-pass Success - Adults/Mixed - Observational } \\
\hline Rocca, 2000 & Ground & Yes ETI & $265 / 331$ & $10 / 21$ & $0.59(0.38,0.93)$ \\
\hline \multicolumn{5}{|c|}{ Subgroup $(\mathrm{I}-\mathrm{squared}=0.0 \%)$} & $0.97(0.71,1.02)$ \\
\hline \multicolumn{6}{|c|}{ First-pass Success - Pediatrics - Observational } \\
\hline Jarvis, 2019 & NR & Mixed & $184 / 313$ & $36 / 61$ & $1.00(0.80,1.26)$ \\
\hline \multicolumn{6}{|c|}{ Overall Success - Adults/Mixed - Observational } \\
\hline Rocca, 2000 & Ground & Yes ETI & $318 / 331$ & $15 / 21$ & $0.74(0.57,0.98)$ \\
\hline Gellerfors, 2014 & Mixed & Advanced & $15 / 23$ & $5 / 5$ & $1.42(0.97,2.08)$ \\
\hline Nwanne, 2020 & Ground & Mixed & $22055 / 27720$ & $2702 / 3486$ & $0.97(0.96,0.99)$ \\
\hline \multicolumn{5}{|c|}{ Subgroup $(\mathrm{I}-\mathrm{squared}=0.0 \%)$} & $0.97(0.89,1.07)$ \\
\hline \multicolumn{6}{|c|}{ Favors cardiac arrest Favors trauma } \\
\hline
\end{tabular}

$\mathrm{CI}=$ confidence interval; $\mathrm{ETI}=$ endotracheal intubation; $\mathrm{KQ}=$ Key Question; $\mathrm{NA}=$ not applicable; $\mathrm{NR}=$ not reported 
Figure H-19. Cardiac arrest versus non-arrest (KQ4) pooled estimate of success

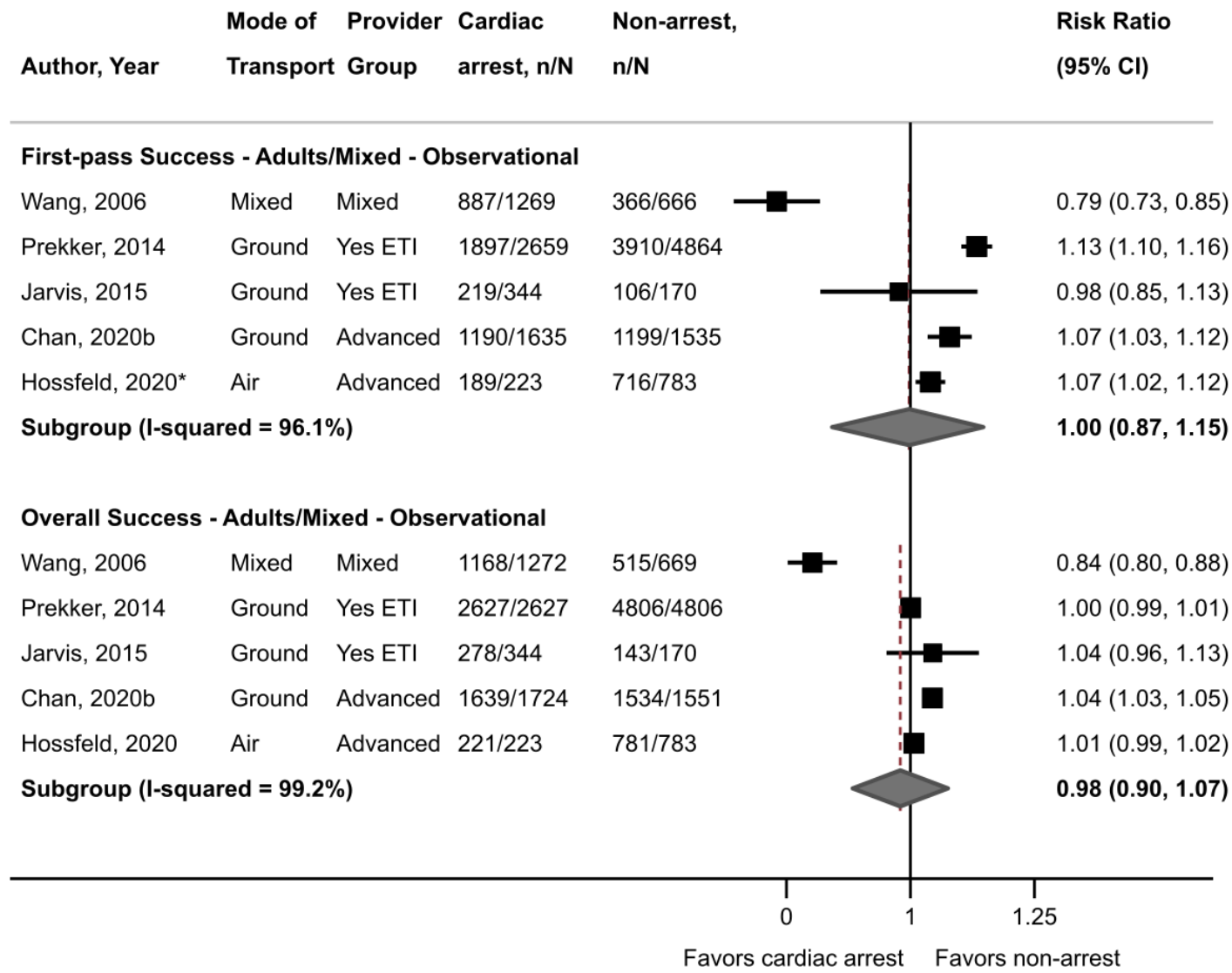

$\mathrm{CI}=$ confidence interval; $\mathrm{KQ}=$ Key Question; $\mathrm{ETI}=$ endotracheal intubation

${ }^{\mathrm{a} A s t e r i s k}$ indicates where adjusted results were used in the analysis 
Figure H-20. Medical versus trauma (KQ4) pooled estimate of success by age and study design

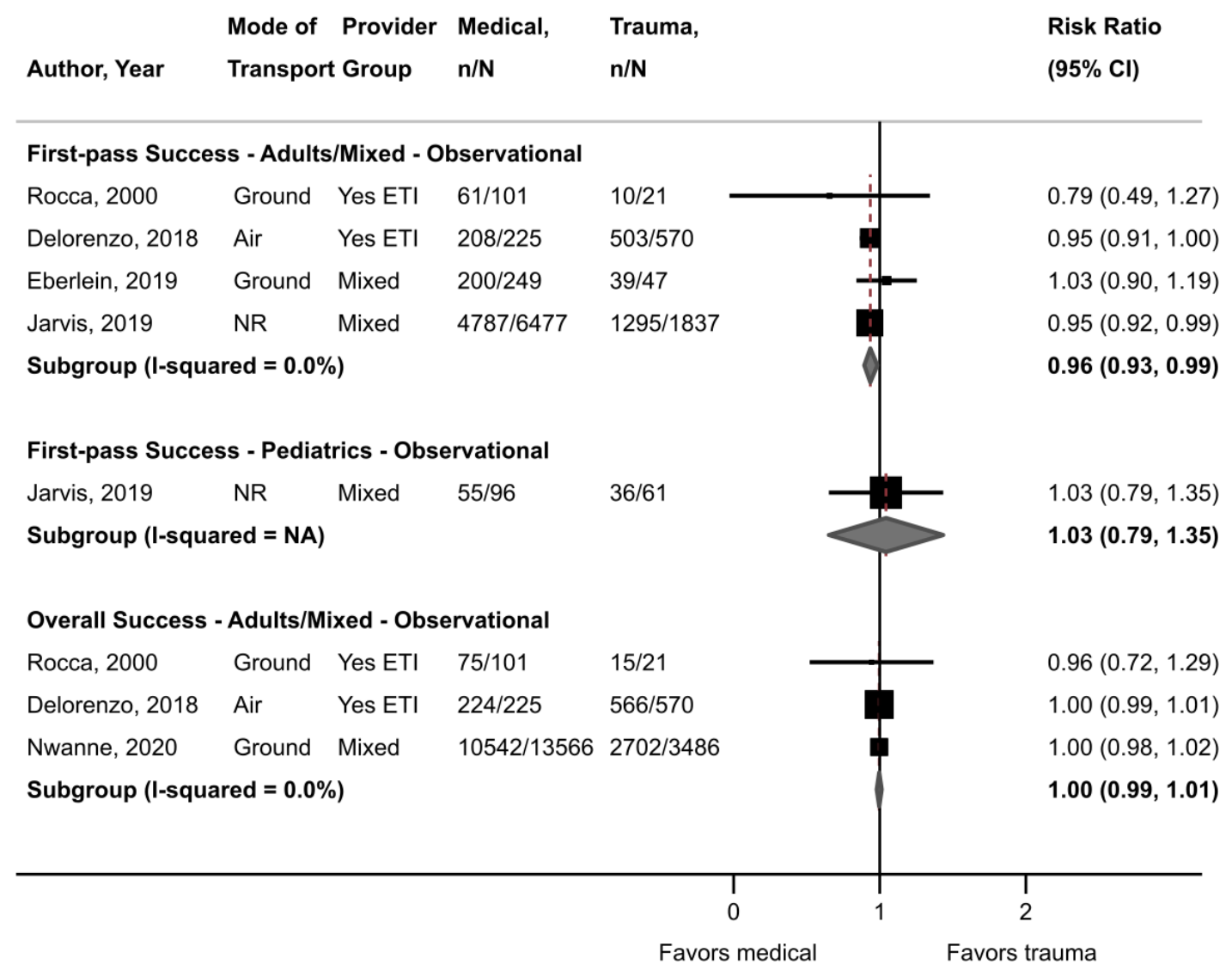

$\mathrm{CI}=$ confidence interval; ETI $=$ endotracheal intubation; $\mathrm{KQ}=$ Key Question; $\mathrm{NA}=$ not applicable; $\mathrm{NR}=$ not reported 


\section{Appendix I. Meta-Analysis: Sensitivity Analyses}

Figure I-1. BVM versus SGA (KQ1) pooled estimate of survival by emergency type, age, and study design; unadjusted

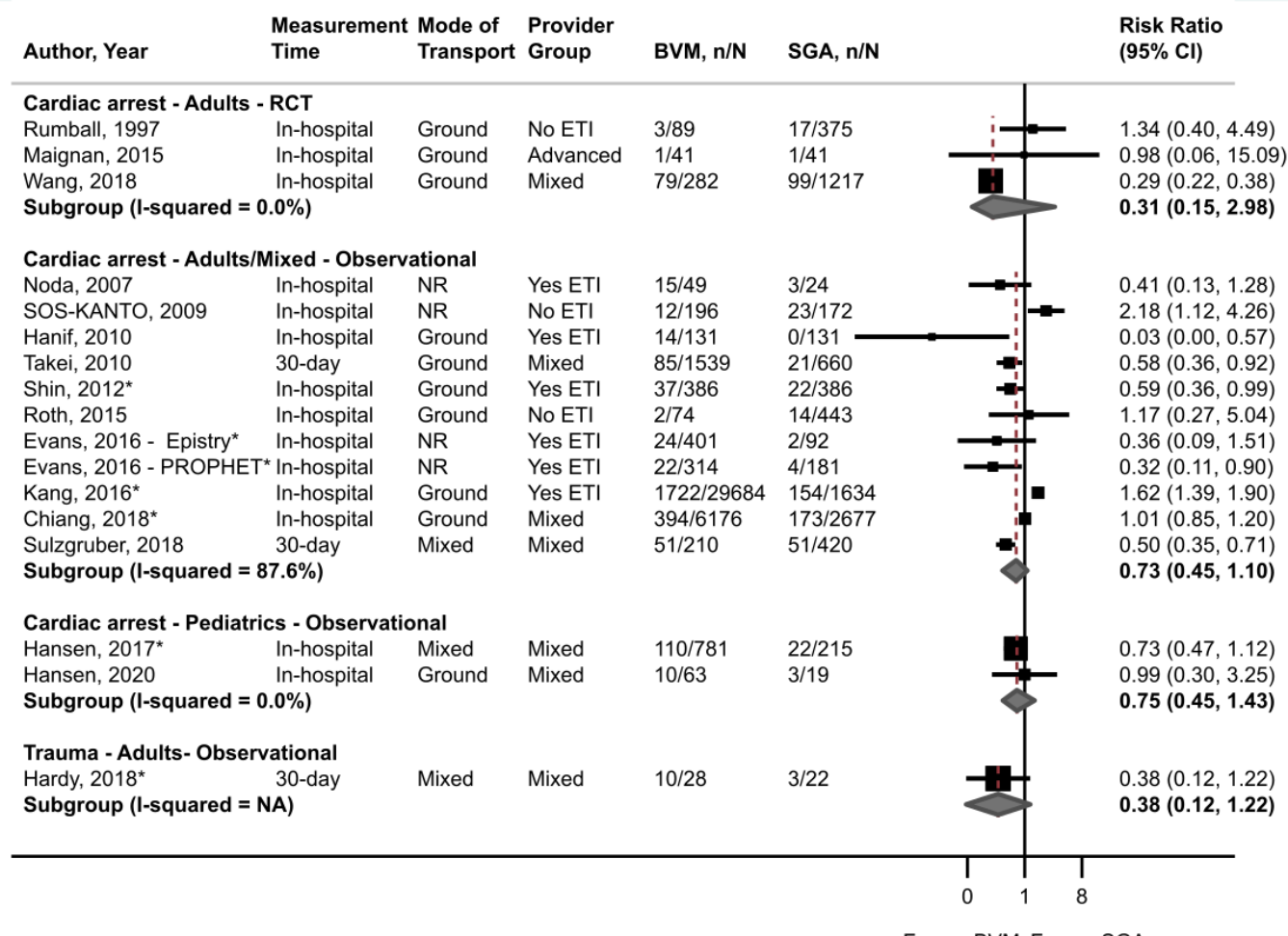

$\mathrm{BVM}=$ bag valve mask; $\mathrm{CI}=$ confidence interval; $\mathrm{ETI}=$ endotracheal intubation; $\mathrm{KQ}=$ Key Question; $\mathrm{NA}=$ not applicable; NR $=$ not reported; $\mathrm{RCT}=$ randomized control trial; $\mathrm{SGA}=$ supraglottic airway

${ }^{a}$ Asterisk indicates where adjusted results were used in the analysis 
Figure I-2. BVM versus SGA (KQ1) pooled estimate of survival by emergency type, age, and study design; excluding Hanif, 2010

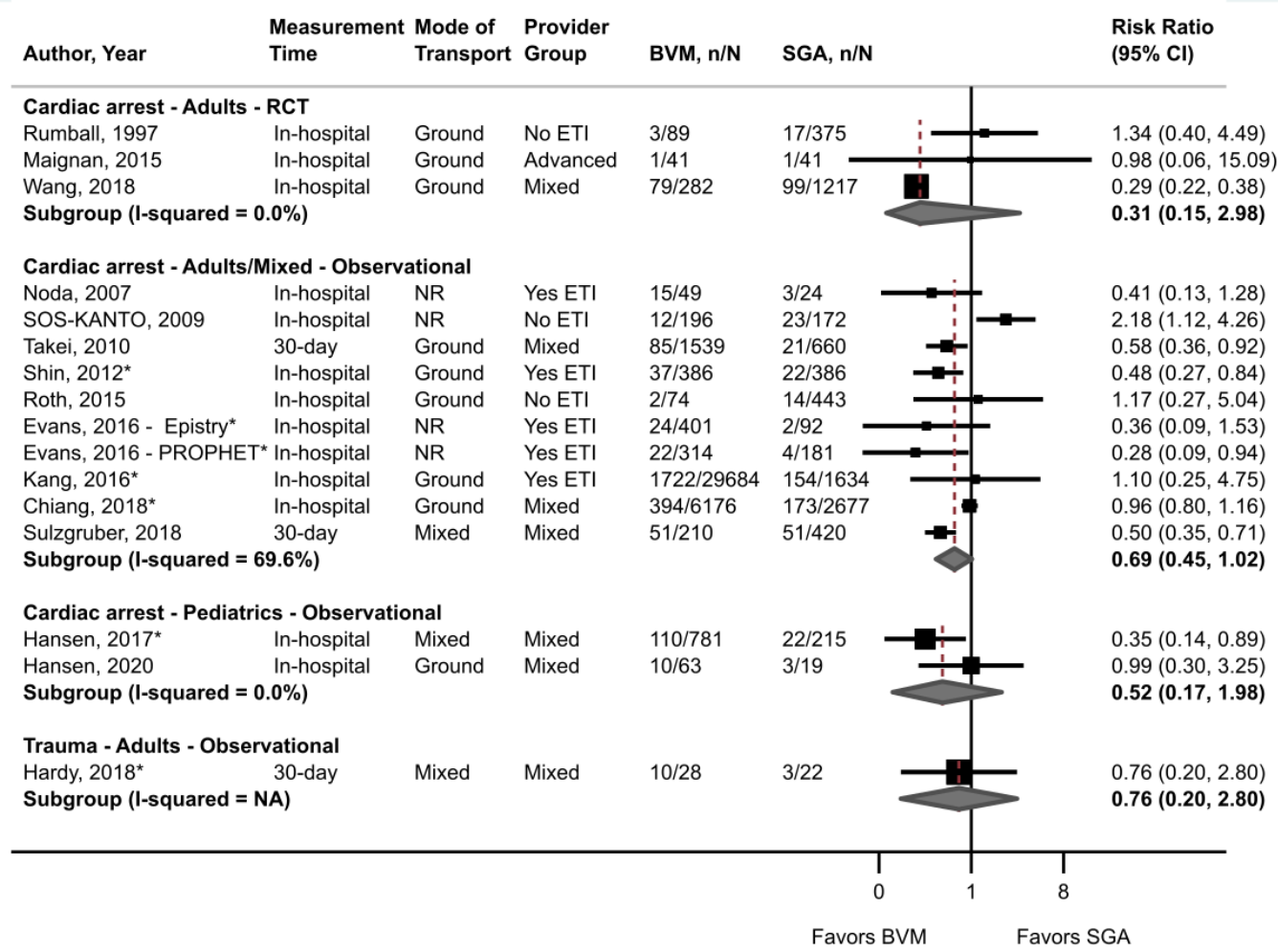

$\mathrm{BVM}=$ bag valve mask; $\mathrm{CI}=$ confidence interval; $\mathrm{ETI}=$ endotracheal intubation; $\mathrm{KQ}=$ Key Question; NA = not applicable; NR $=$ not reported; $\mathrm{RCT}=$ randomized control trial; SGA $=$ supraglottic airway

${ }^{a}$ Asterisk indicates where adjusted results were used in the analysis 
Figure I-3. BVM versus SGA (KQ1) pooled estimate of survival by emergency type, age, and study design; excluding high risk of bias studies

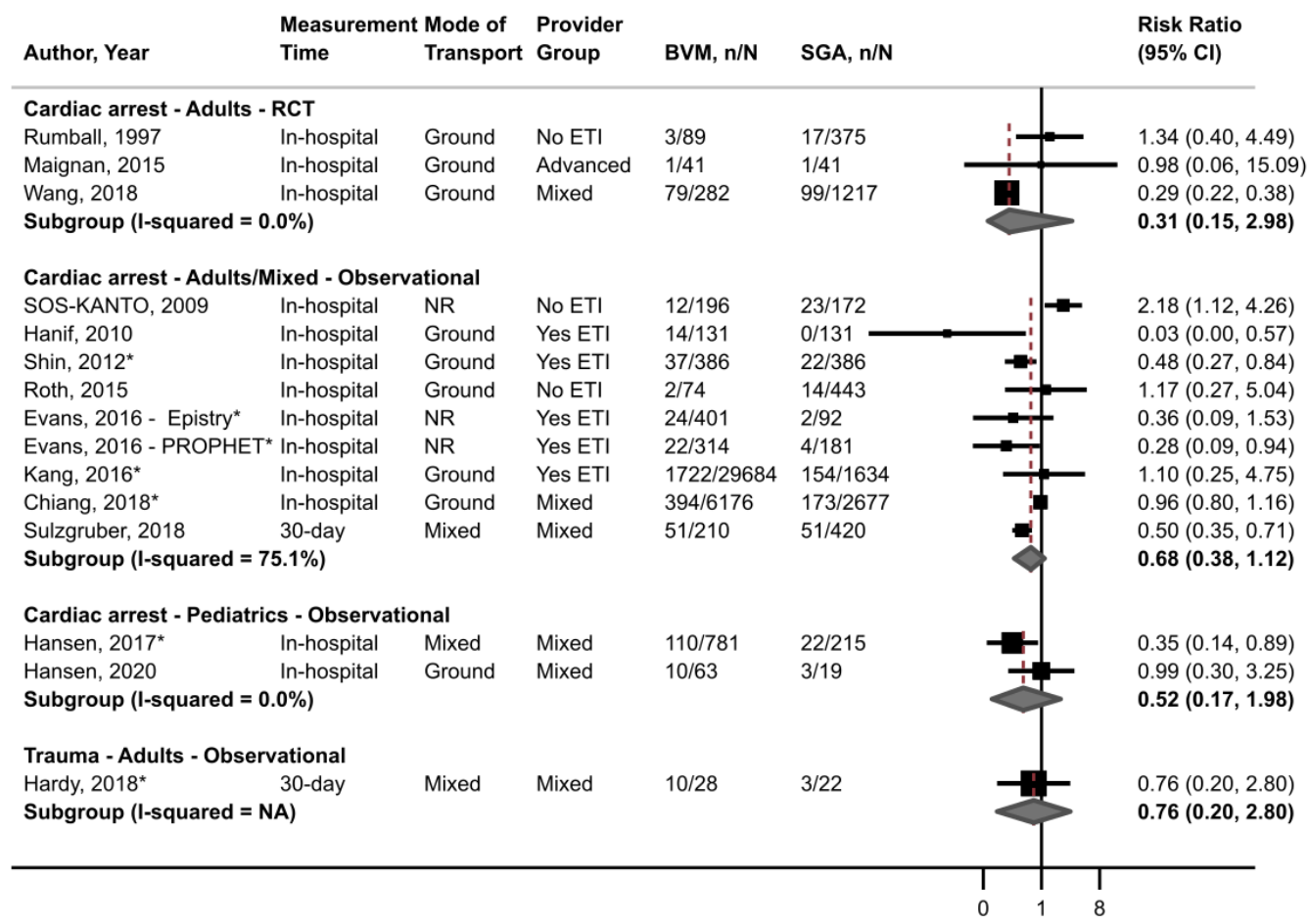

Favors BVM Favors SGA

$\mathrm{BVM}=$ bag valve mask $\mathrm{CI}=$ confidence interval; $\mathrm{ETI}=$ endotracheal intubation; $\mathrm{KQ}=$ Key Question; $\mathrm{NA}=$ not applicable; $\mathrm{NR}$ $=$ not reported; $\mathrm{RCT}=$ randomized control trial; $\mathrm{SGA}=$ supraglottic airway

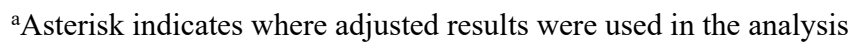


Figure I-4. BVM versus SGA (KQ1) pooled estimate of survival by emergency types, age, and study design; excluding the mixed age studies

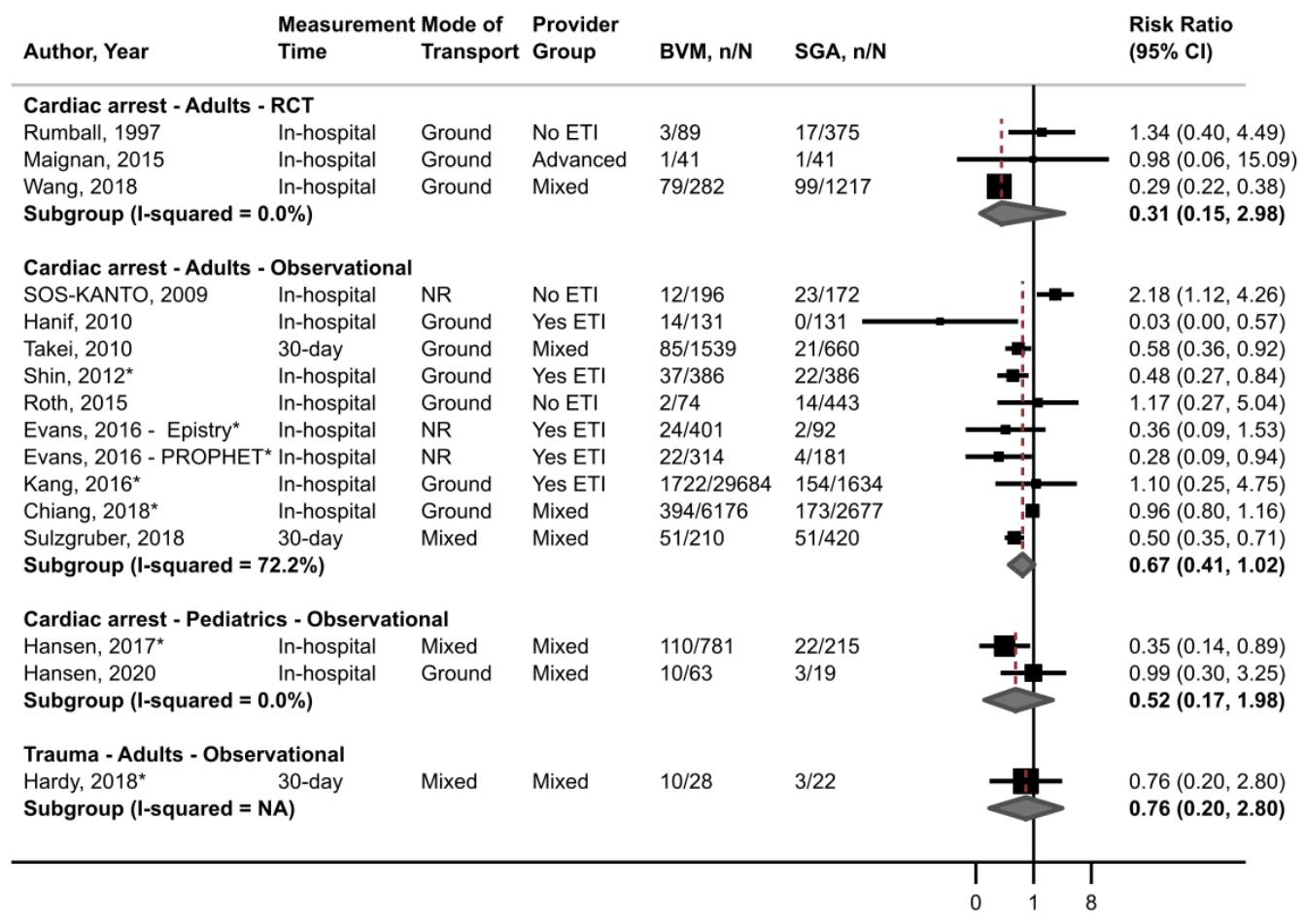

Favors BVM Favors SGA

$\mathrm{BVM}=$ bag valve mask $\mathrm{CI}=$ confidence interval; $\mathrm{ETI}=$ endotracheal intubation; $\mathrm{KQ}=$ Key Question; $\mathrm{NA}=$ applicable; $\mathrm{NR}=$ not reported; $\mathrm{RCT}=$ randomized control trial; $\mathrm{SGA}=$ supraglottic airway

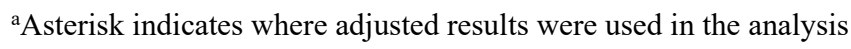


Figure I-5. BVM versus SGA (KQ1) pooled estimate of neurological function by emergency types, age, and study design; unadjusted

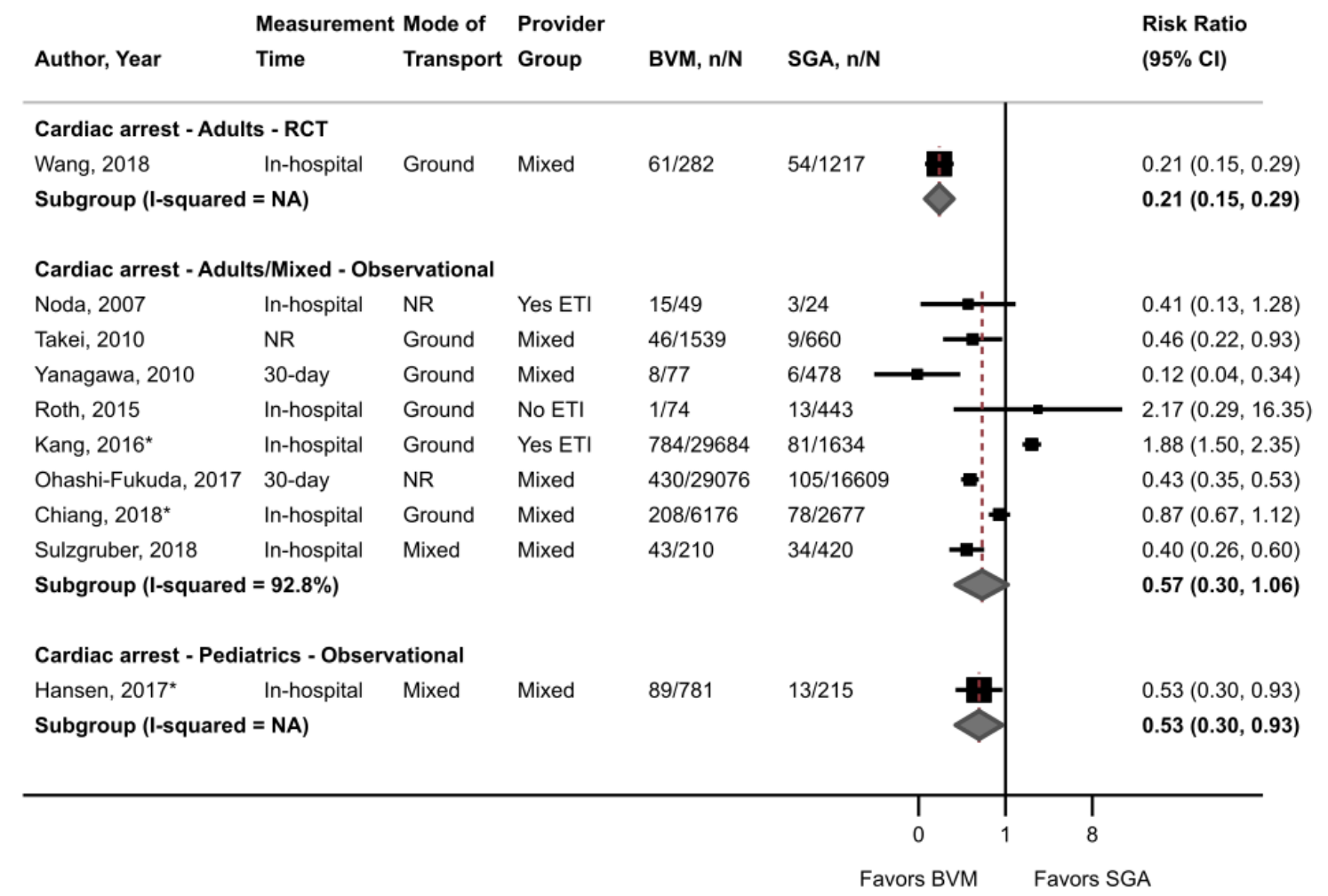

$\mathrm{BVM}=$ bag valve mask $\mathrm{CI}=$ confidence interval; $\mathrm{ETI}=$ endotracheal intubation; $\mathrm{KQ}=$ Key Question; $\mathrm{NA}=$ applicable; $\mathrm{NR}=$ not reported; $\mathrm{RCT}=$ randomized control trial; $\mathrm{SGA}=$ supraglottic airway

${ }^{a}$ Asterisk indicates where adjusted results were used in the analysis 
Figure I-6. BVM versus SGA (KQ1) pooled estimate of neurological function by emergency types, age, and study design; excluding high risk of bias studies

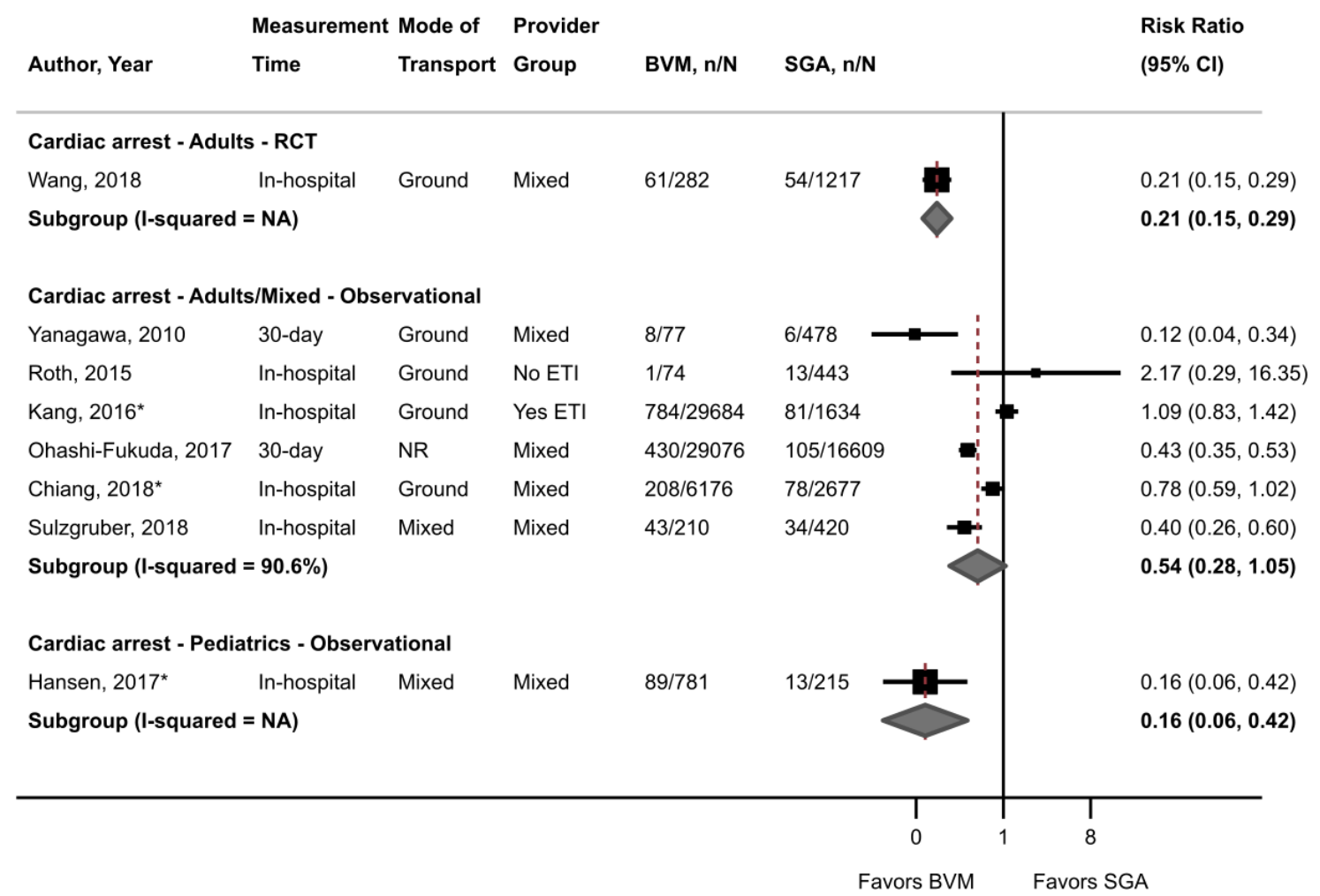

$\mathrm{BVM}=$ bag valve mask $\mathrm{CI}=$ confidence interval; $\mathrm{ETI}=$ endotracheal intubation; $\mathrm{KQ}=$ Key Question; $\mathrm{NA}=$ applicable; $\mathrm{NR}=$ not reported; $\mathrm{RCT}=$ randomized control trial; $\mathrm{SGA}=$ supraglottic airway

${ }^{a}$ Asterisk indicates where adjusted results were used in the analysis 
Figure I-7. BVM versus SGA (KQ1) pooled estimate of ROSC by emergency types, age, and study design; unadjusted

\begin{tabular}{|c|c|c|c|c|c|c|}
\hline \multirow[b]{2}{*}{ Author, Year } & \multicolumn{2}{|c|}{ Measurement Mode of } & \multicolumn{3}{|l|}{ Provider } & \multirow{2}{*}{$\begin{array}{l}\text { Risk Ratio } \\
(95 \% \mathrm{Cl})\end{array}$} \\
\hline & Time & Transport & Group & $\mathrm{BVM}, \mathrm{n} / \mathrm{N}$ & SGA, n/N & \\
\hline \multicolumn{7}{|c|}{ Cardiac arrest - Adults - RCT } \\
\hline Maignan, 2015 & Prehospital & Ground & Advanced & $9 / 41$ & $7 / 41$ & $0.78(0.32,1.89)$ \\
\hline Fiala, 2017 & Not specified & Ground & No ETI & $7 / 41$ & $9 / 35$ & $1.51(0.63,3.63)$ \\
\hline Wang, 2018 & In-hospital & Ground & Mixed & $107 / 282$ & $432 / 1217$ & $0.94(0.79,1.11)$ \\
\hline \multicolumn{6}{|c|}{ Subgroup (I-squared = $0.0 \%)$} & $0.94(0.76,1.27)$ \\
\hline \multicolumn{7}{|c|}{ Cardiac arrest - Adults - Observational } \\
\hline SOS-KANTO, 2009 & ED & NR & No ETI & $80 / 200$ & $67 / 173$ & $0.97(0.75,1.25)$ \\
\hline Takei, 2010 & Sustained & Ground & Mixed & $327 / 1539$ & $133 / 660$ & $0.95(0.79,1.14)$ \\
\hline Yanagawa, 2010 & Prehospital & Ground & Mixed & $13 / 77$ & $37 / 478-$ & $0.46(0.26,0.82)$ \\
\hline Chien, 2012 & Not specified & Ground & No ETI & $32 / 89$ & $147 / 309$ & $1.32(0.98,1.79)$ \\
\hline Nagao, 2012 & Overall & Ground & Yes ETI & $16 / 156$ & $36 / 189$ & $1.86(1.07,3.22)$ \\
\hline Roth, 2015* & Sustained & Ground & No ETI & $5 / 74$ & $61 / 443$ & $2.04(0.85,4.90)$ \\
\hline Kang, 2016 & Prehospital & Ground & Yes ETI & $920 / 29684$ & $87 / 1634$ & $1.72(1.39,2.13)$ \\
\hline Chiang, 2018* & Sustained & Ground & Mixed & $1327 / 6176$ & $649 / 2677$ & $1.13(1.04,1.22)$ \\
\hline Sulzgruber, 2018 & Sustained & Mixed & Mixed & $77 / 210$ & $113 / 420$ & $0.73(0.58,0.93)$ \\
\hline \multicolumn{6}{|c|}{ Subgroup (I-squared = 88.4\%) } & $1.10(0.84,1.46)$ \\
\hline \multicolumn{7}{|c|}{ Cardiac arrest - Pediatrics - Observational } \\
\hline Hansen, 2017* & Sustained & Mixed & Mixed & $141 / 781$ & $58 / 215$ & $1.49(1.15,1.95)$ \\
\hline \multicolumn{6}{|c|}{ Subgroup (I-squared = NA) } & $1.49(1.15,1.95)$ \\
\hline & & & & & & \\
\hline
\end{tabular}

Favors BVM Favors SGA

$\mathrm{BVM}=$ bag valve mask; $\mathrm{CI}=$ confidence interval $\mathrm{ED}=$ emergency department $\mathrm{ETI}=$ endotracheal intubation; $\mathrm{KQ}=\mathrm{Key}$ Question; NA = applicable; NR = not reported; RCT = randomized control trial; ROSC = return of spontaneous circulation; SGA = supraglottic airway

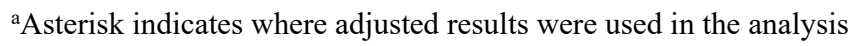


Figure I-8. SGA versus ETI (KQ3) pooled estimate of neurological function by emergency type, age, and study design; as treated

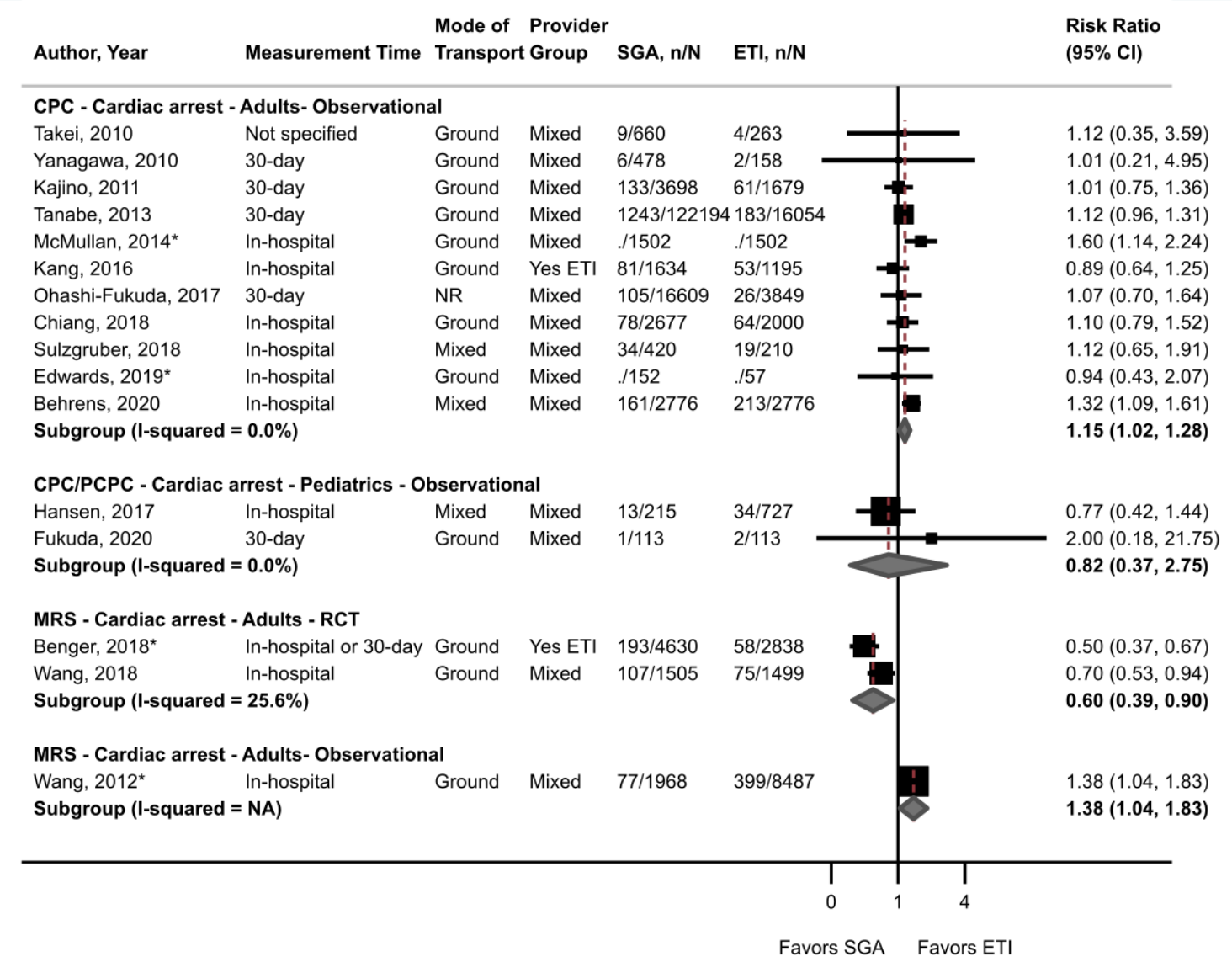

$\mathrm{CI}=$ confidence interval $\mathrm{CPC}=$ Cerebral Performance Category; $\mathrm{ETI}=$ endotracheal intubation; $\mathrm{KQ}=\mathrm{Key}$ Question; $\mathrm{MRS}=$ Modified Rankin Scale; NA = not applicable; NR = not reported; PCPC = Pediatric Cerebral Performance Category; RCT = randomized control trial; SGA = supraglottic airway

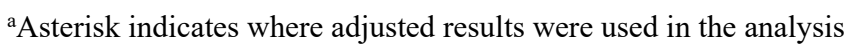


Figure I-9. RSI versus no medication (KQ4) pooled estimate success by age and study design; excluding high risk of bias studies

\begin{tabular}{|c|c|c|c|c|c|}
\hline $\begin{array}{l}\text { Study Design an } \\
\text { Author, Year }\end{array}$ & $\begin{array}{l}\text { d Mode of } \\
\text { Transport }\end{array}$ & $\begin{array}{l}\text { Provider } \\
\text { Group }\end{array}$ & $\begin{array}{l}\text { No medicati } \\
\mathrm{n} / \mathrm{N}\end{array}$ & $R S I, n / N$ & $\begin{array}{l}\text { Risk Ratio } \\
(95 \% \mathrm{Cl})\end{array}$ \\
\hline \multicolumn{6}{|c|}{ First-pass Success - Cardiac arrest - Adults - Observational } \\
\hline Kwok, 2013 & Ground & Yes ETI & $1660 / 2341$ & $273 / 435$ & $0.89(0.82,0.96)$ \\
\hline \multicolumn{5}{|c|}{ Subgroup (I-squared = NA) } & $0.89(0.82,0.96)$ \\
\hline \multicolumn{6}{|c|}{ First-pass Success - Trauma - Adults/Mixed - Observational } \\
\hline Vilke, 1994 & Air & Mixed & $120 / 134$ & $117 / 133$ & $0.98(0.90,1.07)$ \\
\hline Powell, 2019 & Air & Advanced & $10 / 13$ & $188 / 244$ & $1.22(0.83,1.79)$ \\
\hline \multicolumn{5}{|c|}{ Subgroup (I-squared $=0.0 \%)$} & $0.99(0.89,1.23)$ \\
\hline \multicolumn{6}{|c|}{ First-pass Success - Mixed Emergency - Adults/Mixed - Observational } \\
\hline Prekker, 2014 & Ground & Yes ETI & $2490 / 3491$ & $3317 / 4032$ & $1.15(1.12,1.18)$ \\
\hline Sunde, $2015^{\star}$ & Air & Advanced & .190 &.$/ 1110$ & $1.03(0.93,1.13)$ \\
\hline \multicolumn{5}{|c|}{ Subgroup (I-squared $=0.0 \%)$} & $1.14(0.94,1.26)$ \\
\hline \multicolumn{6}{|c|}{ Overall Success - Cardiac arrest - Adults - Observational } \\
\hline Kwok, 2013 & Ground & Yes ETI & $2309 / 2341$ & $425 / 435$ & $0.99(0.98,1.01)$ \\
\hline \multicolumn{5}{|c|}{ Subgroup $(1-$ squared $=\mathrm{NA})$} & $0.99(0.98,1.01)$ \\
\hline \multicolumn{6}{|c|}{ Overall Success - Trauma - Adults - Observational } \\
\hline Bendinelli, 2018 & NR & Yes ETI & $20 / 91$ & $164 / 192$ & $3.89(2.63,5.75)$ \\
\hline \multicolumn{5}{|c|}{ Subgroup (I-squared = NA) } & $3.89(2.63,5.75)$ \\
\hline \multicolumn{6}{|c|}{ Overall Success - Mixed Emergency - Adults/Mixed - Observational } \\
\hline Prekker, 2014 & Ground & Yes ETI & $3431 / 3431$ & $4002 / 4002$ & $1.01(1.00,1.02)$ \\
\hline \multicolumn{5}{|c|}{ Subgroup (I-squared $=$ NA) } & $1.01(1.00,1.02)$ \\
\hline & & & & & \\
\hline & & & & 0 & \\
\hline
\end{tabular}

$\mathrm{CI}=$ confidence interval; ETI $=$ endotracheal intubation; KQ = Key Question; NA = not applicable; NR = not reported; RSI = rapid sequence intubation

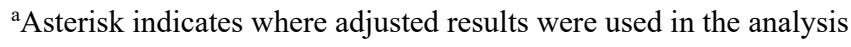


Figure I-10. RSI versus no medication (KQ4) pooled estimate success by age and study design; excluding outlier studies

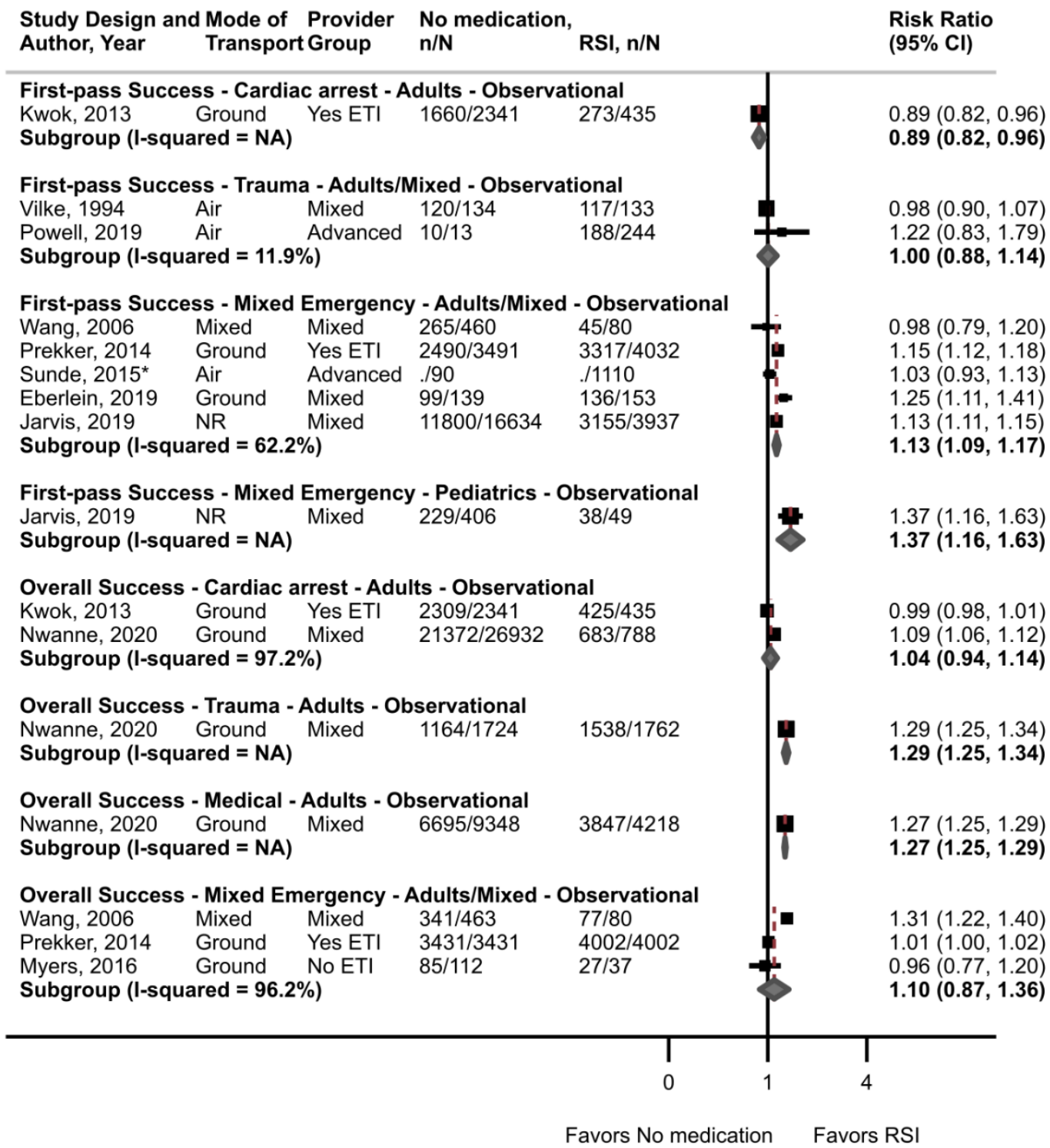

$\mathrm{CI}=$ confidence interval; ETI $=$ endotracheal intubation; $\mathrm{KQ}=$ Key Question; $\mathrm{NA}=$ not applicable; $\mathrm{NR}=$ not reported; RSI = rapid sequence intubation

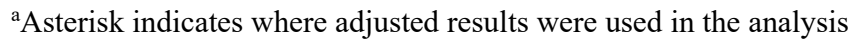


Figure I-11. SGA versus ETI (KQ3) pooled estimate of neurological function by scale, emergency type, age, and study design; per protocol

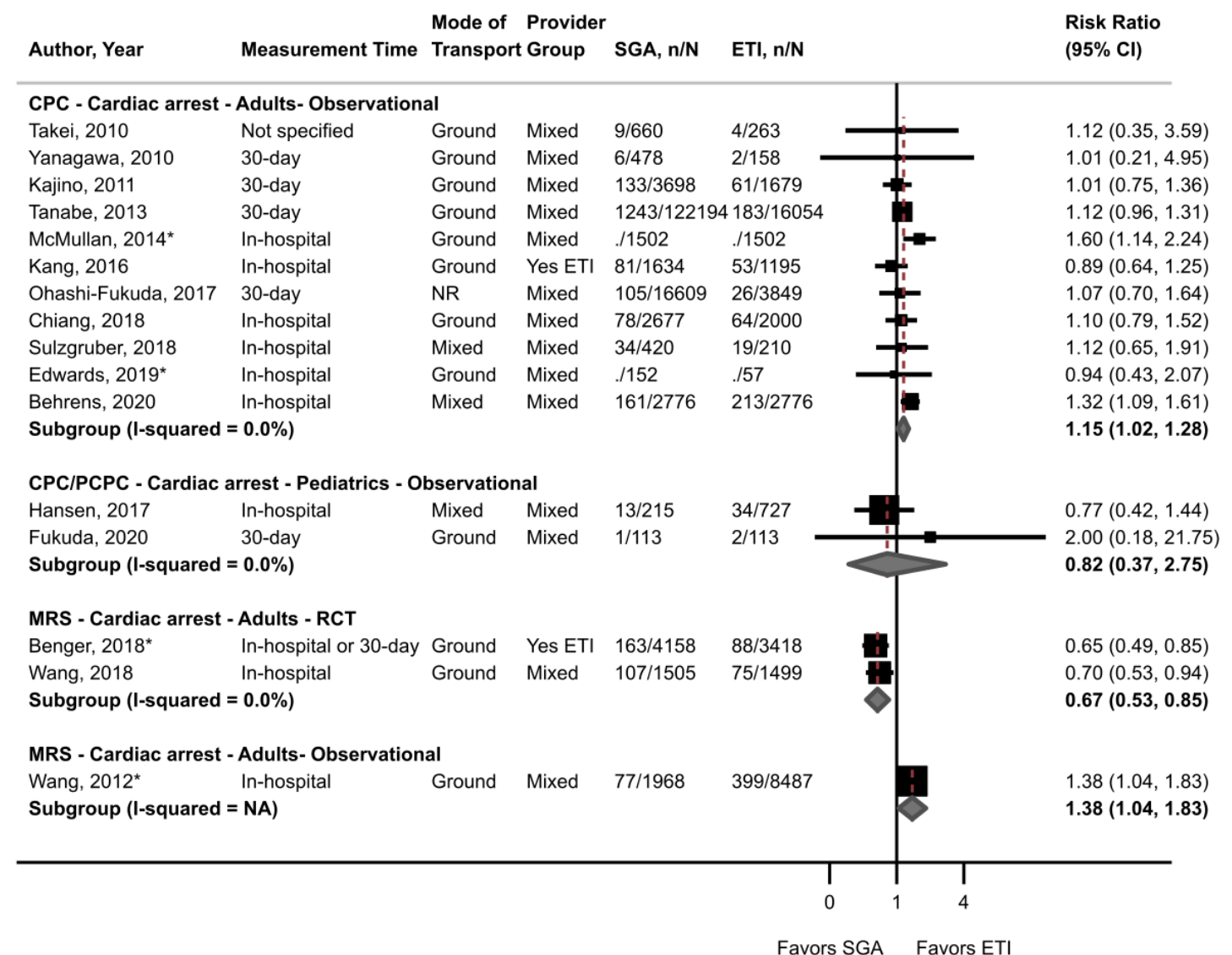

$\mathrm{CI}=$ confidence interval $\mathrm{CPC}=$ Cerebral Performance Category; $\mathrm{ETI}=$ endotracheal intubation; $\mathrm{KQ}=\mathrm{Key}$ Question; $\mathrm{MRS}=$ Modified Rankin Scale; NA = not applicable; NR = not reported; PCPC = Pediatric Cerebral Performance Category; RCT = randomized control trial; $\mathrm{SGA}=$ supraglottic airway

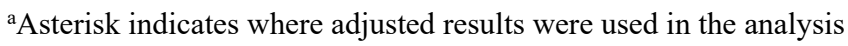


Figure I-12. Cardiac arrest versus medical (KQ4) pooled estimate of success by age and study design; excluding mixed age groups

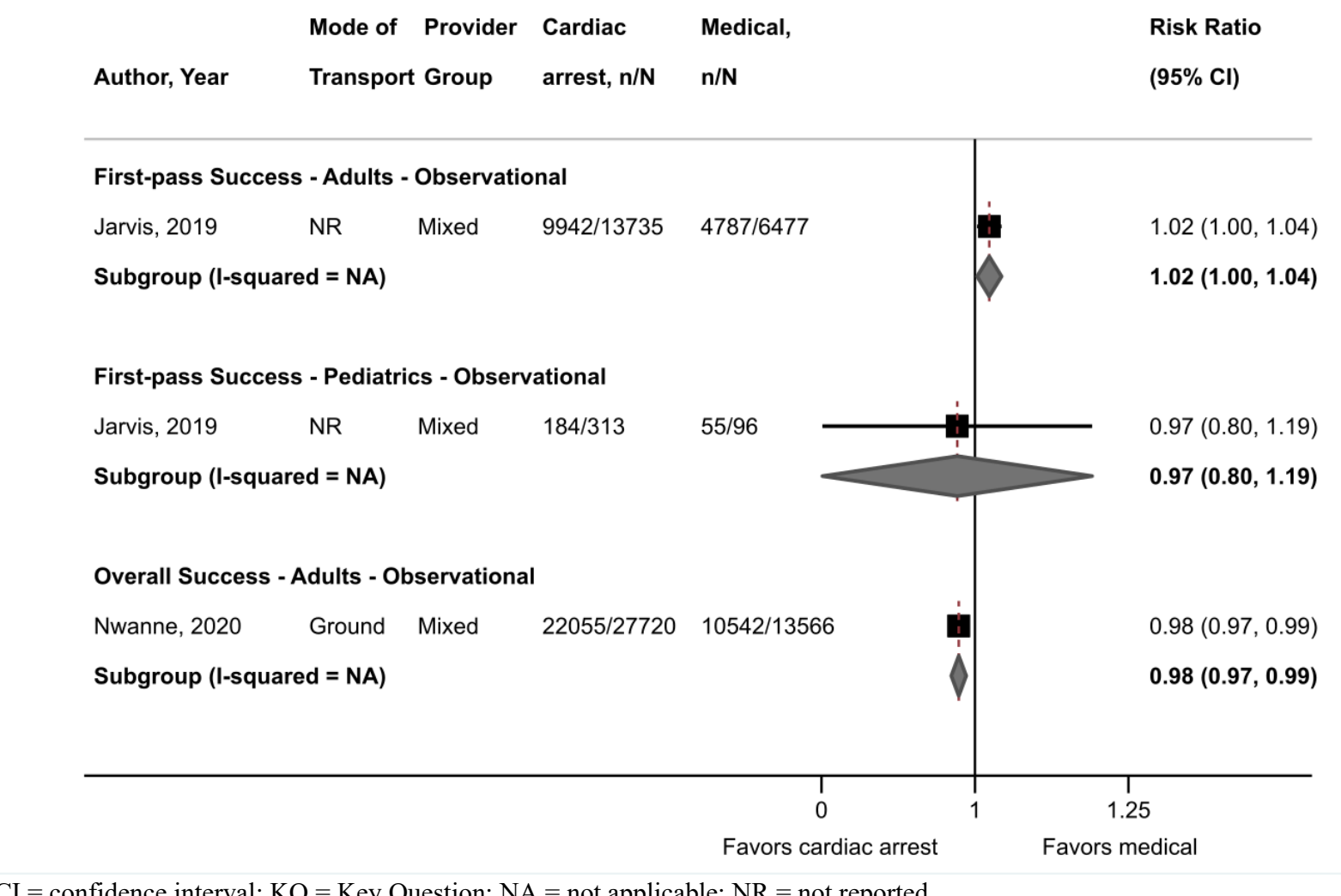

$\mathrm{CI}=$ confidence interval $\mathrm{KQ}=$ Key Question; $\mathrm{NA}=$ not applicable; $\mathrm{NR}=$ not reported 
Figure I-13. Cardiac arrest versus non-arrest (KQ4) pooled estimate of success by age and study design; excluding high risk of bias/mixed age groups

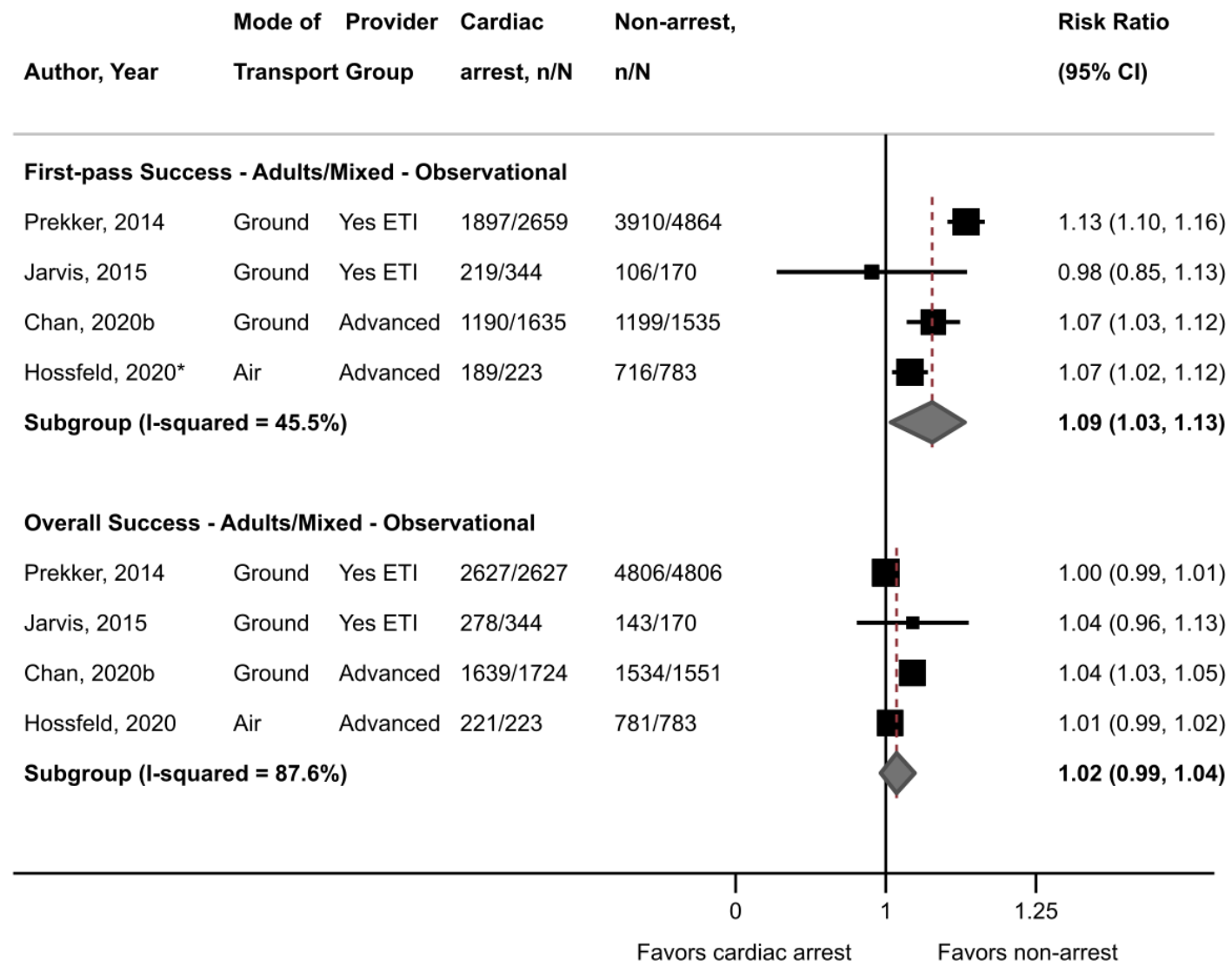

$\mathrm{CI}=$ confidence interval $\mathrm{KQ}=$ Key Question; $\mathrm{ETI}=$ endotracheal intubation

${ }^{a}$ Asterisk indicates where adjusted results were used in the analysis 


\section{Appendix J. Strength of Evidence}

Table J-1. Strength of evidence: Key Question 1

\begin{tabular}{|c|c|c|c|c|c|c|c|c|c|}
\hline $\begin{array}{c}\text { KQ: } \\
\text { Comparison } \\
\text { Outcome }\end{array}$ & Subgroup & $\begin{array}{c}\text { Number of Studies } \\
\text { (Combined N) }\end{array}$ & $\begin{array}{c}\text { Study } \\
\text { Limitations } \\
\text { (Low, } \\
\text { Medium, } \\
\text { High) }\end{array}$ & $\begin{array}{l}\text { Directness } \\
\text { (Direct, } \\
\text { Indirect) }\end{array}$ & $\begin{array}{l}\text { Consistency } \\
\text { (Consistent, } \\
\text { Inconsistent, } \\
\text { Unknown) }\end{array}$ & $\begin{array}{l}\text { Precision } \\
\text { (Precise, } \\
\text { Imprecise) }\end{array}$ & $\begin{array}{c}\text { Reporting } \\
\text { Bias } \\
\text { (Not } \\
\text { Detected, } \\
\text { Possible, } \\
\text { Suspected) }\end{array}$ & Conclusion & $\begin{array}{l}\text { Strength of } \\
\text { Evidence } \\
\text { Grade } \\
\text { (Insufficient, } \\
\text { Low, } \\
\text { Moderate, } \\
\text { High) }\end{array}$ \\
\hline \multirow[t]{3}{*}{$\begin{array}{l}\text { KQ1: BVM vs. } \\
\text { SGA } \\
\text { Survival }\end{array}$} & $\begin{array}{l}\text { Cardiac arrest: } \\
\text { Adults/Mixed } \\
\text { Ages }\end{array}$ & $\begin{array}{l}3 \mathrm{RCT}^{1-3} \\
11 \mathrm{OBS}^{4-13} \\
\\
(49,103)\end{array}$ & Medium & Direct & Consistent & Imprecise & Not detected & No difference in survival & Low \\
\hline & $\begin{array}{l}\text { Cardiac arrest: } \\
\text { Pediatrics }\end{array}$ & $\begin{array}{l}2 \mathrm{OBS}^{14,15} \\
(1,078)\end{array}$ & Medium & Direct & Consistent & Imprecise & Not detected & No difference in survival & Low \\
\hline & $\begin{array}{l}\text { Trauma: } \\
\text { Adults }\end{array}$ & $\begin{array}{l}1 \mathrm{OBS}^{16} \\
(50)\end{array}$ & Medium & Direct & Unknown & Insufficient & Not detected & No conclusion & Insufficient \\
\hline \multirow{2}{*}{$\begin{array}{l}\text { KQ1: BVM vs. } \\
\text { SGA } \\
\text { Neurological } \\
\text { function }\end{array}$} & $\begin{array}{l}\text { Cardiac arrest: } \\
\text { Adults/Mixed } \\
\text { Ages }\end{array}$ & $\begin{array}{l}1 \mathrm{RCT}^{3} \\
8 \mathrm{OBS}^{4,7-9,12,13,17,18} \\
(91,329)\end{array}$ & Medium & Indirect & Inconsistent & Imprecise & Not detected & $\begin{array}{l}\text { Favors BVM in good } \\
\text { neurological function }\end{array}$ & Low \\
\hline & $\begin{array}{l}\text { Cardiac arrest: } \\
\text { Pediatrics }\end{array}$ & $\begin{array}{l}1 \text { OBS }^{15} \\
(996)\end{array}$ & Medium & Indirect & Unknown & Insufficient & Not detected & No conclusion & Insufficient \\
\hline \multirow[t]{2}{*}{$\begin{array}{l}\text { KQ1: BVM vs. } \\
\text { SGA } \\
\text { ROSC }\end{array}$} & $\begin{array}{l}\text { Cardiac arrest: } \\
\text { Adults }\end{array}$ & $\begin{array}{l}3 \mathrm{RCT}^{1,3,19} \\
9 \mathrm{OBS}^{4,7,9,11-13,18,20,21} \\
(46,845)\end{array}$ & Medium & Direct & Inconsistent & Imprecise & Not detected & No difference in ROSC & Low \\
\hline & $\begin{array}{l}\text { Cardiac arrest: } \\
\text { Pediatrics }\end{array}$ & $\begin{array}{l}1 \mathrm{OBS}^{15} \\
(996)\end{array}$ & Medium & Direct & Unknown & Insufficient & Not detected & No conclusion & Insufficient \\
\hline
\end{tabular}




\begin{tabular}{|c|c|c|c|c|c|c|c|c|c|}
\hline $\begin{array}{c}\text { KQ: } \\
\text { Comparison } \\
\text { Outcome } \\
\end{array}$ & Subgroup & $\begin{array}{c}\text { Number of Studies } \\
\text { (Combined N) }\end{array}$ & $\begin{array}{c}\text { Study } \\
\text { Limitations } \\
\text { (Low, } \\
\text { Medium, } \\
\text { High) } \\
\end{array}$ & $\begin{array}{l}\text { Directness } \\
\text { (Direct, } \\
\text { Indirect) }\end{array}$ & $\begin{array}{c}\text { Consistency } \\
\text { (Consistent, } \\
\text { Inconsistent, } \\
\text { Unknown) }\end{array}$ & $\begin{array}{l}\text { Precision } \\
\text { (Precise, } \\
\text { Imprecise) }\end{array}$ & \begin{tabular}{|c} 
Reporting \\
Bias \\
(Not \\
Detected, \\
Possible, \\
Suspected) \\
\end{tabular} & Conclusion & $\begin{array}{c}\text { Strength of } \\
\text { Evidence } \\
\text { Grade } \\
\text { (Insufficient, } \\
\text { Low, } \\
\text { Moderate, } \\
\text { High) }\end{array}$ \\
\hline $\begin{array}{l}\text { KQ1: BVM vs. } \\
\text { SGA }\end{array}$ & Length of stay & $\begin{array}{l}1 \mathrm{OBS}^{16} \\
(50)\end{array}$ & Medium & Direct & Unknown & Insufficient & Not detected & No conclusion & Insufficient \\
\hline \multirow[t]{3}{*}{$\begin{array}{l}\text { Qualitative } \\
\text { Analysis }\end{array}$} & $\begin{array}{l}\text { Successful } \\
\text { airway }\end{array}$ & $\begin{array}{l}1 \mathrm{RCT}^{19} \\
1 \mathrm{OBS}^{9} \\
(593)\end{array}$ & Medium & Indirect & Inconsistent & Insufficient & Not detected & No conclusion & Insufficient \\
\hline & $\begin{array}{l}\text { Oxygenation/ } \\
\text { Ventilation }\end{array}$ & $\begin{array}{l}2 \mathrm{RCT}^{2,19} \\
3 \mathrm{OBS}^{11,12,20} \\
(2,525)\end{array}$ & Medium & Direct & Consistent & Insufficient & Not detected & $\begin{array}{l}\text { No difference in oxygenation } \\
\text { and ventilation }\end{array}$ & Moderate \\
\hline & Harms & $\begin{array}{l}2 \mathrm{RCT}^{1,19} \\
2 \mathrm{OBS}^{9,14} \\
(696)\end{array}$ & Medium & Direct & Consistent & Insufficient & Not detected & No difference in harms & Moderate \\
\hline
\end{tabular}


Table J-2. Strength of evidence: Key Question 2

\begin{tabular}{|c|c|c|c|c|c|c|c|c|c|}
\hline $\begin{array}{c}\text { KQ: } \\
\text { Comparison } \\
\text { Outcome } \\
\end{array}$ & Subgroup & $\begin{array}{c}\text { Number of } \\
\text { Studies } \\
\text { (Combined N) }\end{array}$ & $\begin{array}{c}\text { Study } \\
\text { Limitations } \\
\text { (Low, Medium, } \\
\text { High) } \\
\end{array}$ & $\begin{array}{c}\text { Directness } \\
\text { (Direct, } \\
\text { Indirect) } \\
\end{array}$ & $\begin{array}{l}\text { Consistency } \\
\text { (Consistent, } \\
\text { Inconsistent, } \\
\text { Unknown) }\end{array}$ & $\begin{array}{l}\text { Precision } \\
\text { (Precise, } \\
\text { Imprecise) } \\
\end{array}$ & $\begin{array}{l}\text { Reporting } \\
\text { Bias } \\
\text { (Not } \\
\text { Detected, } \\
\text { Possible, } \\
\text { Suspected) } \\
\end{array}$ & Conclusion & $\begin{array}{l}\text { Strength of } \\
\text { Evidence } \\
\text { Grade } \\
\text { (Insufficient, } \\
\text { Low, } \\
\text { Moderate, } \\
\text { High) }\end{array}$ \\
\hline \multirow[t]{4}{*}{$\begin{array}{l}\text { KQ2 BVM vs. } \\
\text { ETI }\end{array}$} & $\begin{array}{l}\text { Cardiac Arrest: } \\
\text { Adults/Mixed Ages }\end{array}$ & $\begin{array}{l}2 \mathrm{RCT}^{3,22-24} \\
9 \mathrm{OBS}^{4-8,10,12,13} \\
(48,629)\end{array}$ & Medium & Direct & Inconsistent & Imprecise & Not detected & No difference & Moderate \\
\hline & $\begin{array}{l}\text { Cardiac Arrest: } \\
\text { Pediatrics }\end{array}$ & $\begin{array}{l}1 \mathrm{RCT}^{25,26} \\
2 \mathrm{OBS}^{14,15} \\
(2,235)\end{array}$ & Low & Direct & Inconsistent & Imprecise & Not detected & No difference & Low \\
\hline & $\begin{array}{l}\text { Trauma: } \\
\text { Adults/Mixed Ages }\end{array}$ & $\begin{array}{l}1 \mathrm{RCT}^{27} \\
2 \mathrm{OBS}^{28,29} \\
(1,328)\end{array}$ & Low & Direct & Inconsistent & Imprecise & Not detected & No difference & Low \\
\hline & $\begin{array}{l}\text { Trauma: } \\
\text { Pediatrics }\end{array}$ & $\begin{array}{l}\text { No RCT } \\
1 \mathrm{OBS}^{30} \\
(578) \\
\end{array}$ & High & Direct & Unknown & Imprecise & Not detected & No conclusion & Insufficient \\
\hline \multirow[t]{2}{*}{$\begin{array}{l}\text { KQ2: BVM vs. } \\
\text { ETI } \\
\text { Neurological } \\
\text { Function }\end{array}$} & $\begin{array}{l}\text { Cardiac Arrest: } \\
\text { Adults }\end{array}$ & $\begin{array}{l}1 \mathrm{RCT}^{22,23} \\
6 \mathrm{OBS}^{4,7,12,13,17,18} \\
(76,477)\end{array}$ & Low & Direct & Inconsistent & Precise & Not detected & No difference & Moderate \\
\hline & $\begin{array}{l}\text { Cardiac Arrest: } \\
\text { Pediatrics }\end{array}$ & $\begin{array}{l}1 \mathrm{RCT}^{25,26} \\
1 \mathrm{OBS}^{15} \\
(2,099) \\
\end{array}$ & Medium & Direct & Inconsistent & Imprecise & Not detected & No difference & Low \\
\hline \multirow[t]{2}{*}{$\begin{array}{l}\text { KQ2: BVM vs. } \\
\text { ETI }\end{array}$} & $\begin{array}{l}\text { Cardiac Arrest: } \\
\text { Adults }\end{array}$ & $\begin{array}{l}2 \mathrm{RCT}^{3,22-24} \\
8 \\
\mathrm{OBS}^{4,6,7,12,13,18,21}, \\
31 \\
(47,244) \\
\end{array}$ & Low & Direct & Inconsistent & Imprecise & Not detected & No difference & Low \\
\hline & $\begin{array}{l}\text { Cardiac Arrest: } \\
\text { Pediatrics }\end{array}$ & $\begin{array}{l}1 \mathrm{OBS}^{15} \\
(1,508)\end{array}$ & Low & Direct & Unknown & Precise & Not detected & No difference & Low \\
\hline
\end{tabular}




\begin{tabular}{|c|c|c|c|c|c|c|c|c|c|}
\hline $\begin{array}{c}\text { KQ: } \\
\text { Comparison } \\
\text { Outcome }\end{array}$ & Subgroup & $\begin{array}{c}\text { Number of } \\
\text { Studies } \\
\text { (Combined N) }\end{array}$ & $\begin{array}{c}\text { Study } \\
\text { Limitations } \\
\text { (Low, Medium, } \\
\text { High) }\end{array}$ & $\begin{array}{l}\text { Directness } \\
\text { (Direct, } \\
\text { Indirect) }\end{array}$ & $\begin{array}{l}\text { Consistency } \\
\text { (Consistent, } \\
\text { Inconsistent, } \\
\text { Unknown) }\end{array}$ & $\begin{array}{l}\text { Precision } \\
\text { (Precise, } \\
\text { Imprecise) }\end{array}$ & $\begin{array}{c}\text { Reporting } \\
\text { Bias } \\
\text { (Not } \\
\text { Detected, } \\
\text { Possible, } \\
\text { Suspected) }\end{array}$ & Conclusion & $\begin{array}{c}\text { Strength of } \\
\text { Evidence } \\
\text { Grade } \\
\text { (Insufficient, } \\
\text { Low, } \\
\text { Moderate, } \\
\text { High) }\end{array}$ \\
\hline \multirow[t]{5}{*}{$\begin{array}{l}\text { KQ2: BVM vs. } \\
\text { ETI } \\
\text { Qualitative } \\
\text { Analysis }\end{array}$} & Length of stay & $\begin{array}{l}1 \mathrm{RCT}^{27} \\
1 \mathrm{CCT}^{25,26} \\
(1,142) \\
\end{array}$ & Low & Indirect & Consistent & Imprecise & Not detected & $\begin{array}{l}\text { No difference in ICU or } \\
\text { Hospital LOS }\end{array}$ & Low \\
\hline & Successful airway & $\begin{array}{l}1 \mathrm{RCT}^{22} \\
1 \mathrm{CCT}^{25,26} \\
(2,873)\end{array}$ & Low & Indirect & Inconsistent & Imprecise & Not detected & No conclusion & Insufficient \\
\hline & $\begin{array}{l}\text { Oxygenation/ } \\
\text { Ventilation }\end{array}$ & $\begin{array}{l}1 \mathrm{RCT}^{27} \\
1 \mathrm{OBS}^{12} \\
(2,535) \\
\end{array}$ & Low & Indirect & Consistent & Imprecise & Not detected & No difference & Low \\
\hline & $\begin{array}{l}\text { Survival to } \\
\text { hospital admission }\end{array}$ & $\begin{array}{l}1 \mathrm{RCT}^{22} \\
3 \mathrm{OBS}^{6,10,15} \\
(10,339) \\
\end{array}$ & Medium & Direct & Inconsistent & Imprecise & Not detected & No difference & Low \\
\hline & \begin{tabular}{|l|} 
Neurological \\
Function (not in \\
Meta-analyses)
\end{tabular} & $\begin{array}{l}1 \mathrm{RCT}^{3,24} \\
2 \mathrm{OBS}^{8,30}\end{array}$ & High & Direct & Inconsistent & Imprecise & Not detected & No conclusion & Insufficient \\
\hline
\end{tabular}


Table J-3. Strength of evidence: Key Question 3

\begin{tabular}{|c|c|c|c|c|c|c|c|c|c|}
\hline $\begin{array}{c}\text { KQ: } \\
\text { Comparison } \\
\text { Outcome } \\
\end{array}$ & Subgroup & $\begin{array}{c}\text { Number of Studies } \\
\text { (Combined N) }\end{array}$ & $\begin{array}{c}\text { Study } \\
\text { Limitations } \\
\text { (Low, Medium, } \\
\text { High) } \\
\end{array}$ & $\begin{array}{l}\text { Directness } \\
\text { (Direct, } \\
\text { Indirect) } \\
\end{array}$ & $\begin{array}{l}\text { Consistency } \\
\text { (Consistent, } \\
\text { Inconsistent, } \\
\text { Unknown) }\end{array}$ & $\begin{array}{l}\text { Precision } \\
\text { (Precise, } \\
\text { Imprecise) } \\
\end{array}$ & $\begin{array}{l}\text { Reporting } \\
\text { Bias } \\
\text { (Not } \\
\text { Detected, } \\
\text { Possible, } \\
\text { Suspected) }\end{array}$ & Conclusion & $\begin{array}{c}\text { Strength of } \\
\text { Evidence } \\
\text { Grade } \\
\text { (Insufficient, } \\
\text { Low, } \\
\text { Moderate, } \\
\text { High) } \\
\end{array}$ \\
\hline \multirow[t]{3}{*}{$\begin{array}{l}\text { KQ3: SGA } \\
\text { vs. ETI } \\
\text { Survival }\end{array}$} & Cardiac Arrest: Adults & $\begin{array}{l}3 \mathrm{RCT}^{3,32,33} \\
13 \mathrm{OBS}^{4-8,10,12,13,34-37} \\
(177,088)\end{array}$ & Low & Direct & Inconsistent & Precise & Not detected & No difference & Low \\
\hline & $\begin{array}{l}\text { Cardiac Arrest: } \\
\text { Pediatrics }\end{array}$ & $\begin{array}{l}3 \mathrm{OBS}^{14,15,38} \\
(1,260)\end{array}$ & Medium & Direct & Inconsistent & Precise & Not detected & No difference & Low \\
\hline & Trauma: Adults & $\begin{array}{l}1 \mathrm{OBS}^{39} \\
(2,344)\end{array}$ & Medium & Direct & NA & Precise & Not detected & No Conclusion & Insufficient \\
\hline $\begin{array}{l}\text { KQ3: SGA } \\
\text { vs. ETI } \\
\text { Neurological } \\
\text { function - } \\
\text { mRS }\end{array}$ & Cardiac Arrest: Adults & $\begin{array}{l}2 \mathrm{RCT}^{3,32} \\
1 \mathrm{OBS}^{40} \\
(22,748)\end{array}$ & Medium & Direct & Inconsistent & Precise & Not detected & No difference & Low \\
\hline \multirow{2}{*}{$\begin{array}{l}\text { KQ3: SGA } \\
\text { vs. ETI } \\
\text { Neurological } \\
\text { function - } \\
\text { CPC/PCPC }\end{array}$} & $\begin{array}{l}\text { Cardiac Arrest: Adults } \\
\text { (CPC) }\end{array}$ & $\begin{array}{l}11 \mathrm{OBS}^{4,7,12,13,17,18,34-} \\
37,41 \\
(179,330)\end{array}$ & Medium & Direct & Inconsistent & Precise & Not detected & Difference favoring ETI & Low \\
\hline & \begin{tabular}{|l} 
Cardiac Arrest: \\
Pediatrics (PCPC)
\end{tabular} & $\begin{array}{l}2 \mathrm{OBS}^{15,38} \\
(1,168)\end{array}$ & Medium & Direct & Consistent & Imprecise & Not detected & No difference & Low \\
\hline \multirow[t]{2}{*}{$\begin{array}{l}\text { KQ3: SGA } \\
\text { vs. ETI } \\
\text { ROSC }\end{array}$} & Cardiac Arrest: Adults & $\begin{array}{l}3 \mathrm{RCT}^{3,32,33} \\
13 \mathrm{OBS}^{4,7,12,13,18,21,35-} \\
37,40,42,43 \\
(185,474)\end{array}$ & Medium & Direct & Inconsistent & Precise & Not detected & $\begin{array}{l}\text { Difference favoring } \\
\text { SGA }\end{array}$ & Low \\
\hline & $\begin{array}{l}\text { Cardiac Arrest: } \\
\text { Pediatrics }\end{array}$ & $\begin{array}{l}2 \mathrm{OBS}^{15,38} \\
(1,168)\end{array}$ & Low & Direct & Inconsistent & Imprecise & Not detected & No difference & Low \\
\hline \multirow[t]{2}{*}{$\begin{array}{l}\text { KQ3: SGA } \\
\text { vs. ETI } \\
\text { First-pass } \\
\text { Success }\end{array}$} & Cardiac Arrest: Adults & $\begin{array}{l}1 \mathrm{RCT}^{3} \\
4 \mathrm{OBS}^{43-46} \\
(23,535)\end{array}$ & Moderate & Indirect & Inconsistent & Precise & Not detected & $\begin{array}{l}\text { Difference favoring } \\
\text { SGA }\end{array}$ & Low \\
\hline & $\begin{array}{l}\text { Cardiac Arrest: } \\
\text { Pediatrics }\end{array}$ & $\begin{array}{l}2 \mathrm{OBS}^{14,46} \\
(445)\end{array}$ & High & Indirect & Consistent & Precise & Not detected & $\begin{array}{l}\text { Difference favoring } \\
\text { SGA }\end{array}$ & Low \\
\hline
\end{tabular}




\begin{tabular}{|c|c|c|c|c|c|c|c|c|c|}
\hline $\begin{array}{c}\text { KQ: } \\
\text { Comparison } \\
\text { Outcome } \\
\end{array}$ & Subgroup & $\begin{array}{c}\text { Number of Studies } \\
\text { (Combined N) }\end{array}$ & $\begin{array}{c}\text { Study } \\
\text { Limitations } \\
\text { (Low, Medium, } \\
\text { High) } \\
\end{array}$ & $\begin{array}{l}\text { Directness } \\
\text { (Direct, } \\
\text { Indirect) }\end{array}$ & $\begin{array}{l}\text { Consistency } \\
\text { (Consistent, } \\
\text { Inconsistent, } \\
\text { Unknown) }\end{array}$ & $\begin{array}{l}\text { Precision } \\
\text { (Precise, } \\
\text { Imprecise) } \\
\end{array}$ & $\begin{array}{c}\text { Reporting } \\
\text { Bias } \\
\text { (Not } \\
\text { Detected, } \\
\text { Possible, } \\
\text { Suspected) } \\
\end{array}$ & Conclusion & $\begin{array}{c}\text { Strength of } \\
\text { Evidence } \\
\text { Grade } \\
\text { (Insufficient, } \\
\text { Low, } \\
\text { Moderate, } \\
\text { High) } \\
\end{array}$ \\
\hline & Trauma: Adults & $\begin{array}{l}1 \mathrm{OBS}^{46} \\
(2,143) \\
\end{array}$ & High & Indirect & NA & Precise & Not detected & No conclusion & Insufficient \\
\hline & Trauma: Pediatrics & $\begin{array}{l}1 \mathrm{OBS}^{46} \\
(69)\end{array}$ & High & Indirect & NA & Precise & Not detected & No Conclusion & Insufficient \\
\hline & Medical: Adults & $\begin{array}{l}1 \mathrm{RCT}^{47} \\
1 \mathrm{OBS}^{46} \\
(7,501)\end{array}$ & Moderate & Indirect & Inconsistent & Precise & Not detected & No difference & Low \\
\hline & Medical: Pediatrics & $\begin{array}{l}1 \mathrm{OBS}^{46} \\
(100)\end{array}$ & High & Indirect & NA & Precise & Not detected & No conclusion & Insufficient \\
\hline & $\begin{array}{l}\text { Mixed Emergency } \\
\text { Types: Adults }\end{array}$ & $\begin{array}{l}2 \mathrm{OBS}^{48,49} \\
(407) \\
\end{array}$ & Moderate & Indirect & Consistent & Precise & Not detected & $\begin{array}{l}\text { Difference favoring } \\
\text { SGA }\end{array}$ & Low \\
\hline \multirow{4}{*}{$\begin{array}{l}\text { KQ3: SGA } \\
\text { vs. ETI } \\
\text { Overall } \\
\text { Success }\end{array}$} & Cardiac Arrest: Adults & $\begin{array}{l}3 \mathrm{RCT}^{32,33,40} \\
6 \mathrm{OBS}^{43-45,50-52} \\
(48,703) \\
\end{array}$ & Moderate & Indirect & Inconsistent & Precise & Not detected & No difference & Moderate \\
\hline & Trauma: Adults & $\begin{array}{l}1 \mathrm{OBS}^{52} \\
(3,824)\end{array}$ & High & Indirect & NA & Precise & Not detected & No conclusion & Insufficient \\
\hline & Medical: Adults & $\begin{array}{l}1 \mathrm{RCT}^{47} \\
2 \mathrm{OBS}^{52,53} \\
(15,848)\end{array}$ & Moderate & Indirect & Inconsistent & Precise & Not detected & No difference & Moderate \\
\hline & $\begin{array}{l}\text { Mixed emergency types: } \\
\text { Adults }\end{array}$ & $\begin{array}{l}3 \mathrm{OBS}^{48,54,55} \\
(3,267) \\
\end{array}$ & Moderate & Indirect & Consistent & Precise & Not detected & No difference & Moderate \\
\hline $\begin{array}{l}\text { KQ3: SGA } \\
\text { vs. ETI }\end{array}$ & 24-hour survival & $\begin{array}{l}3 \mathrm{OBS}^{34,40,42} \\
(29,777)\end{array}$ & High & Direct & Inconsistent & Precise & Not detected & No conclusion & Insufficient \\
\hline
\end{tabular}




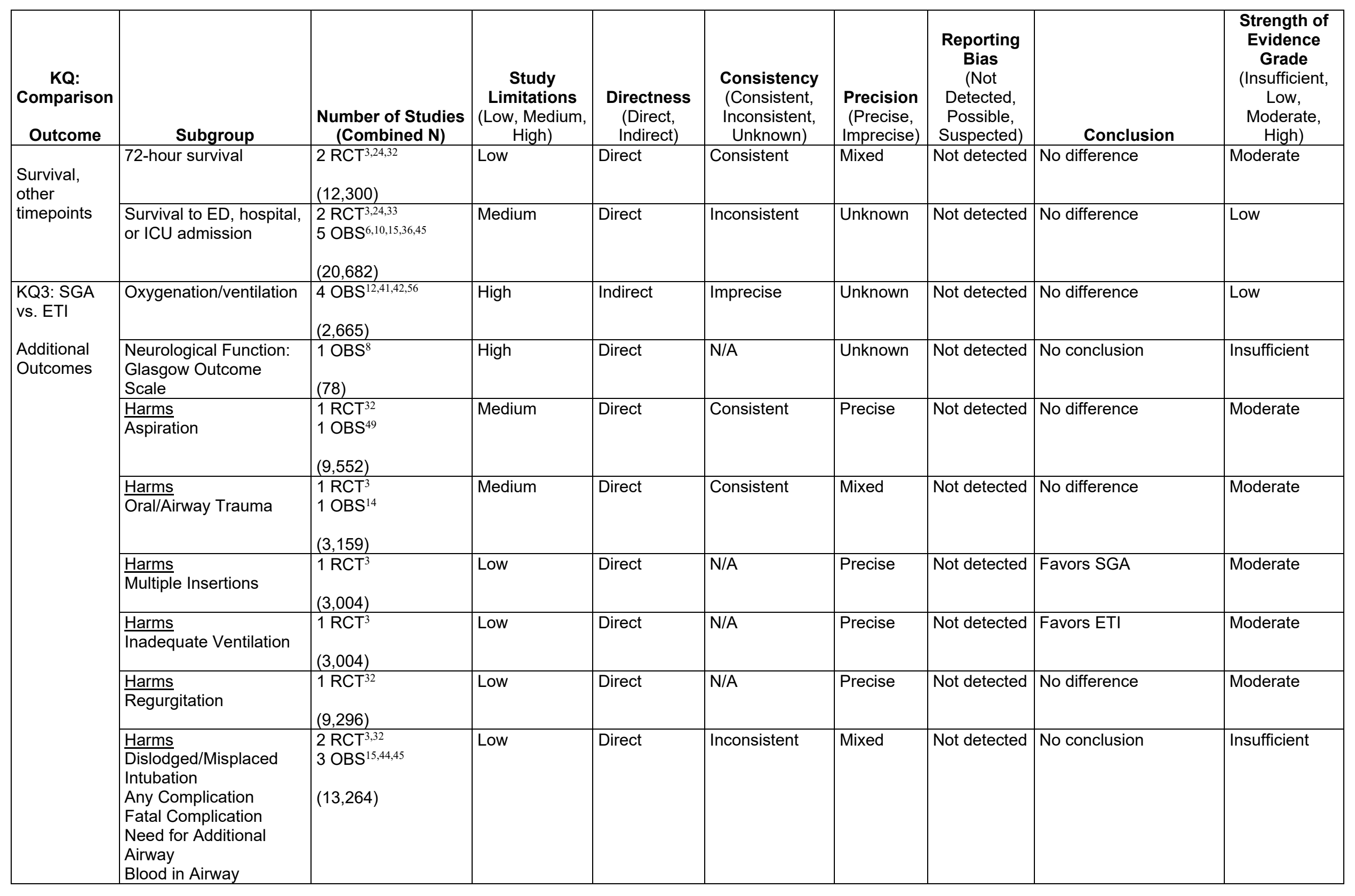


Table J-4. Strength of evidence: Key Question 4

\begin{tabular}{|c|c|c|c|c|c|c|c|c|c|}
\hline $\begin{array}{c}\text { KQ: } \\
\text { Comparison } \\
\text { Outcome }\end{array}$ & Subgroup & $\begin{array}{c}\text { Number of } \\
\text { Studies } \\
\text { (Combined N) }\end{array}$ & $\begin{array}{c}\text { Study } \\
\text { Limitations } \\
\text { (Low, Medium, } \\
\text { High) }\end{array}$ & $\begin{array}{l}\text { Directness } \\
\text { (Direct, } \\
\text { Indirect) }\end{array}$ & $\begin{array}{l}\text { Consistency } \\
\text { (Consistent, } \\
\text { Inconsistent, } \\
\text { Unknown) }\end{array}$ & $\begin{array}{l}\text { Precision } \\
\text { (Precise, } \\
\text { Imprecise) }\end{array}$ & $\begin{array}{c}\text { Reporting } \\
\text { Bias } \\
\text { (Not } \\
\text { Detected, } \\
\text { Possible, } \\
\text { Suspected) }\end{array}$ & Conclusion & $\begin{array}{c}\text { Strength of } \\
\text { Evidence } \\
\text { Grade } \\
\text { (Insufficient, } \\
\text { Low, } \\
\text { Moderate, } \\
\text { High) }\end{array}$ \\
\hline \multirow{3}{*}{$\begin{array}{l}\text { KQ4: RSI vs. } \\
\text { no medication } \\
\text { Survival }\end{array}$} & $\begin{array}{l}\text { Cardiac Arrest: } \\
\text { Adults }\end{array}$ & $\begin{array}{l}1 \text { OBS }^{57} \\
(3,047)\end{array}$ & Medium & Direct & Unknown & Imprecise & Not detected & No conclusion & Insufficient \\
\hline & $\begin{array}{l}\text { Trauma: } \\
\text { Adults/Mixed ages }\end{array}$ & $\begin{array}{l}4 \mathrm{OBS}^{58-61} \\
(2,520) \\
\end{array}$ & Medium & Direct & Inconsistent & Imprecise & Not detected & No difference & Low \\
\hline & Medical: Adults & $\begin{array}{l}1 \mathrm{OBS}^{62} \\
(1,454) \\
\end{array}$ & Medium & Direct & Unknown & Precise & Not detected & No conclusion & Insufficient \\
\hline \multirow{4}{*}{$\begin{array}{l}\text { KQ4: RSI vs. } \\
\text { no medication } \\
\text { First-pass } \\
\text { success }\end{array}$} & $\begin{array}{l}\text { Cardiac Arrest: } \\
\text { Adults }\end{array}$ & $\begin{array}{l}1 \text { OBS }^{57} \\
(2,776)\end{array}$ & Medium & Indirect & Unknown & Precise & Not detected & No conclusion & Insufficient \\
\hline & $\begin{array}{l}\text { Trauma: } \\
\text { Adults/Mixed ages }\end{array}$ & $\begin{array}{l}2 \mathrm{OBS}^{61,63} \\
(530)\end{array}$ & Medium & Indirect & Consistent & Precise & Not detected & No difference & Low \\
\hline & $\begin{array}{l}\text { Mixed emergency: } \\
\text { Adults/Mixed ages }\end{array}$ & $\begin{array}{l}5 \text { OBS }^{46,64-67} \\
(30,126) \\
\end{array}$ & High & Indirect & Consistent & Precise & Not detected & Favors RSI & Low \\
\hline & $\begin{array}{l}\text { Mixed emergency: } \\
\text { Pediatrics }\end{array}$ & $\begin{array}{l}1 \mathrm{OBS}^{46} \\
(455)\end{array}$ & High & Indirect & Unknown & Precise & Not detected & No conclusion & Insufficient \\
\hline \multirow{4}{*}{$\begin{array}{l}\text { KQ4: RSI vs. } \\
\text { no medication } \\
\text { Overall } \\
\text { success }\end{array}$} & $\begin{array}{l}\text { Cardiac Arrest: } \\
\text { Adults }\end{array}$ & $\begin{array}{l}2 \text { OBS }^{52,57} \\
(30,496)\end{array}$ & Medium & Indirect & Inconsistent & Precise & Not detected & No difference & Low \\
\hline & Trauma: Adults & $\begin{array}{l}2 \mathrm{OBS}^{52,59} \\
(3,769) \\
\end{array}$ & Medium & Indirect & Inconsistent & Precise & Not detected & Favors RSI & Low \\
\hline & Medical: Adults & $\begin{array}{l}1 \mathrm{OBS}^{52} \\
(13,566) \\
\end{array}$ & High & Indirect & Unknown & Precise & Not detected & No conclusion & Insufficient \\
\hline & $\begin{array}{l}\text { Mixed emergency: } \\
\text { Adults/Mixed ages }\end{array}$ & $\begin{array}{l}3 \mathrm{OBS}^{64,65,68} \\
(8,215) \\
\end{array}$ & High & Indirect & Consistent & Precise & Not detected & No difference & Low \\
\hline \multirow{2}{*}{$\begin{array}{l}\text { KQ4: RSI vs. } \\
\text { sedation- } \\
\text { facilitated } \\
\\
\text { First-pass } \\
\text { success }\end{array}$} & $\begin{array}{l}\text { Mixed emergency: } \\
\text { Adults/Mixed ages }\end{array}$ & $\begin{array}{l}3 \mathrm{OBS}^{46,64,67} \\
(5,778)\end{array}$ & High & Indirect & Consistent & Imprecise & Not detected & Favors RSI & Low \\
\hline & $\begin{array}{l}\text { Mixed emergency: } \\
\text { Pediatrics }\end{array}$ & $\begin{array}{l}1 \mathrm{OBS}^{46} \\
(64)\end{array}$ & High & Indirect & Unknown & Imprecise & Not detected & No conclusion & Insufficient \\
\hline
\end{tabular}




\begin{tabular}{|c|c|c|c|c|c|c|c|c|c|}
\hline $\begin{array}{c}\text { KQ: } \\
\text { Comparison } \\
\text { Outcome }\end{array}$ & Subgroup & $\begin{array}{c}\text { Number of } \\
\text { Studies } \\
\text { (Combined N) }\end{array}$ & $\begin{array}{c}\text { Study } \\
\text { Limitations } \\
\text { (Low, Medium, } \\
\text { High) }\end{array}$ & $\begin{array}{l}\text { Directness } \\
\text { (Direct, } \\
\text { Indirect) }\end{array}$ & $\begin{array}{l}\text { Consistency } \\
\text { (Consistent, } \\
\text { Inconsistent, } \\
\text { Unknown) }\end{array}$ & $\begin{array}{l}\text { Precision } \\
\text { (Precise, } \\
\text { Imprecise) }\end{array}$ & $\begin{array}{c}\text { Reporting } \\
\text { Bias } \\
\text { (Not } \\
\text { Detected, } \\
\text { Possible, } \\
\text { Suspected) }\end{array}$ & Conclusion & $\begin{array}{c}\text { Strength of } \\
\text { Evidence } \\
\text { Grade } \\
\text { (Insufficient, } \\
\text { Low, } \\
\text { Moderate, } \\
\text { High) }\end{array}$ \\
\hline \multirow{2}{*}{$\begin{array}{l}\text { KQ4: } \\
\text { Sedation- } \\
\text { facilitated vs. } \\
\text { no medication } \\
\text { First-pass } \\
\text { success }\end{array}$} & $\begin{array}{l}\text { Mixed emergency: } \\
\text { Adults/Mixed ages }\end{array}$ & $\begin{array}{l}3 \mathrm{OBS}^{46,64,67} \\
(18,841)\end{array}$ & High & Indirect & Inconsistent & Precise & Not detected & No difference & Low \\
\hline & $\begin{array}{l}\text { Mixed emergency: } \\
\text { Pediatrics }\end{array}$ & $\begin{array}{l}1 \mathrm{OBS}^{46} \\
(421)\end{array}$ & High & Indirect & Unknown & Imprecise & Not detected & No conclusion & Insufficient \\
\hline \multirow{4}{*}{$\begin{array}{l}\text { KQ4: Direct } \\
\text { vs. video } \\
\text { laryngoscopy } \\
\text { First pass } \\
\text { success }\end{array}$} & $\begin{array}{l}\text { Cardiac Arrest: } \\
\text { Adults }\end{array}$ & $\begin{array}{l}2 \mathrm{RCT}^{69,70} \\
3 \mathrm{OBS}^{43,71,72} \\
(905)\end{array}$ & Medium & Indirect & Inconsistent & Precise & Not detected & No difference & Low \\
\hline & Trauma: Adults & $\begin{array}{l}2 \text { OBS }^{63,67} \\
(310)\end{array}$ & Medium & Indirect & Inconsistent & Imprecise & Not detected & No difference & Insufficient \\
\hline & Medical: Adults & $\begin{array}{l}1 \text { OBS }^{67} \\
(249)\end{array}$ & High & Indirect & Unknown & Precise & Not detected & No conclusion & Insufficient \\
\hline & $\begin{array}{l}\text { Mixed emergency: } \\
\text { Adults/Mixed ages }\end{array}$ & $\begin{array}{l}2 \mathrm{RCT}^{73,74} \\
4 \mathrm{OBS}^{71,75-77} \\
(5,816)\end{array}$ & Medium & Indirect & Consistent & Precise & Not detected & No difference & Moderate \\
\hline \multirow{2}{*}{$\begin{array}{l}\text { KQ4: Direct } \\
\text { vs. video } \\
\text { laryngoscopy } \\
\text { Overall } \\
\text { Success }\end{array}$} & $\begin{array}{l}\text { Cardiac Arrest: } \\
\text { Adults }\end{array}$ & $\begin{array}{l}2 \mathrm{RCT}^{69,70} \\
3 \mathrm{OBS}^{43,71,72} \\
(905) \\
\end{array}$ & Medium & Indirect & Consistent & Precise & Not detected & No difference & Moderate \\
\hline & $\begin{array}{l}\text { Mixed emergency: } \\
\text { Adults }\end{array}$ & $\begin{array}{l}3 \mathrm{RCT}^{73,78,79} \\
4 \mathrm{OBS}^{71,75,77,80} \\
(2,155)\end{array}$ & Medium & Indirect & Inconsistent & Precise & Not detected & No difference & Low \\
\hline $\begin{array}{l}\text { KQ4: Medical } \\
\text { vs. cardiac } \\
\text { arrest }\end{array}$ & Adults/Mixed Ages & $\begin{array}{l}2 \mathrm{OBS}^{46,81} \\
(20,644)\end{array}$ & High & Indirect & Inconsistent & Precise & Not detected & No conclusion & Insufficient \\
\hline
\end{tabular}




\begin{tabular}{|c|c|c|c|c|c|c|c|c|c|}
\hline $\begin{array}{c}\text { KQ: } \\
\text { Comparison } \\
\text { Outcome }\end{array}$ & Subgroup & $\begin{array}{c}\text { Number of } \\
\text { Studies } \\
\text { (Combined N) }\end{array}$ & $\begin{array}{c}\text { Study } \\
\text { Limitations } \\
\text { (Low, Medium, } \\
\text { High) }\end{array}$ & $\begin{array}{l}\text { Directness } \\
\text { (Direct, } \\
\text { Indirect) }\end{array}$ & $\begin{array}{l}\text { Consistency } \\
\text { (Consistent, } \\
\text { Inconsistent, } \\
\text { Unknown) }\end{array}$ & $\begin{array}{l}\text { Precision } \\
\text { (Precise, } \\
\text { Imprecise) }\end{array}$ & $\begin{array}{c}\text { Reporting } \\
\text { Bias } \\
\text { (Not } \\
\text { Detected, } \\
\text { Possible, } \\
\text { Suspected) }\end{array}$ & Conclusion & $\begin{array}{c}\text { Strength of } \\
\text { Evidence } \\
\text { Grade } \\
\text { (Insufficient, } \\
\text { Low, } \\
\text { Moderate, } \\
\text { High) }\end{array}$ \\
\hline $\begin{array}{l}\text { KQ4: Medical } \\
\text { vs. cardiac } \\
\text { arrest } \\
\text { Overall } \\
\text { success }\end{array}$ & Adults/Mixed Ages & $\begin{array}{l}2 \mathrm{OBS}^{52,81} \\
(41,718)\end{array}$ & High & Indirect & Consistent & Precise & Not detected & No difference & Low \\
\hline $\begin{array}{l}\text { KQ4: Non- } \\
\text { arrest vs. } \\
\text { cardiac arrest } \\
\text { First pass } \\
\text { success }\end{array}$ & Adults/Mixed Ages & $\begin{array}{l}5 \mathrm{OBS}^{64,65,71,82,83} \\
(14,148)\end{array}$ & High & Indirect & Consistent & Precise & Not detected & No difference & Low \\
\hline $\begin{array}{l}\text { KQ4: Trauma } \\
\text { vs. cardiac } \\
\text { arrest }\end{array}$ & Adults/Mixed Ages & $\begin{array}{l}2 \mathrm{OBS}^{46,81} \\
(15,924)\end{array}$ & High & Indirect & Inconsistent & Precise & Not detected & No conclusion & Insufficient \\
\hline $\begin{array}{l}\text { First pass } \\
\text { success }\end{array}$ & Pediatrics & $\begin{array}{l}1 \mathrm{OBS}^{46} \\
(374)\end{array}$ & High & Indirect & Unknown & Precise & Not detected & No conclusion & Insufficient \\
\hline $\begin{array}{l}\text { KQ4: Trauma } \\
\text { vs. cardiac } \\
\text { arrest } \\
\text { Overall } \\
\text { Success }\end{array}$ & $\begin{array}{l}\text { Adults/ Mixed } \\
\text { Ages }\end{array}$ & $\begin{array}{l}3 \mathrm{OBS}^{52,81,84} \\
(31,586)\end{array}$ & High & Indirect & Inconsistent & Precise & Not detected & No difference & Low \\
\hline $\begin{array}{l}\text { KQ4: Medical } \\
\text { vs. Trauma }\end{array}$ & Adults/Mixed Ages & $\begin{array}{l}4 \mathrm{OBS}^{46,67,81,85} \\
(9,527)\end{array}$ & High & Indirect & Consistent & Precise & Not detected & Favors medical & Low \\
\hline $\begin{array}{l}\text { First pass } \\
\text { success }\end{array}$ & Pediatrics & $\begin{array}{l}1 \mathrm{OBS}^{46} \\
(157)\end{array}$ & High & Indirect & Unknown & Precise & Not detected & No conclusion & Insufficient \\
\hline
\end{tabular}




\begin{tabular}{|c|c|c|c|c|c|c|c|c|c|}
\hline $\begin{array}{c}\text { KQ: } \\
\text { Comparison } \\
\text { Outcome } \\
\end{array}$ & Subgroup & $\begin{array}{c}\text { Number of } \\
\text { Studies } \\
\text { (Combined N) }\end{array}$ & $\begin{array}{c}\text { Study } \\
\text { Limitations } \\
\text { (Low, Medium, } \\
\text { High) } \\
\end{array}$ & \begin{tabular}{|c|} 
Directness \\
(Direct, \\
Indirect)
\end{tabular} & $\begin{array}{l}\text { Consistency } \\
\text { (Consistent, } \\
\text { Inconsistent, } \\
\text { Unknown) }\end{array}$ & $\begin{array}{c}\text { Precision } \\
\text { (Precise, } \\
\text { Imprecise) }\end{array}$ & $\begin{array}{l}\text { Reporting } \\
\text { Bias } \\
\text { (Not } \\
\text { Detected, } \\
\text { Possible, } \\
\text { Suspected) }\end{array}$ & Conclusion & $\begin{array}{c}\text { Strength of } \\
\text { Evidence } \\
\text { Grade } \\
\text { (Insufficient, } \\
\text { Low, } \\
\text { Moderate, } \\
\text { High) }\end{array}$ \\
\hline $\begin{array}{l}\text { KQ4: Medical } \\
\text { vs. Trauma } \\
\text { Overall } \\
\text { Success }\end{array}$ & Adult/Mixed Ages & $\begin{array}{l}3 \mathrm{OBS}^{52,81,85} \\
(17,969)\end{array}$ & High & Indirect & Consistent & Precise & Not detected & No difference & Low \\
\hline
\end{tabular}




\section{Appendix J References}

1. Maignan M, Koch FX, Kraemer M, et al. Impact of laryngeal tube use on chest compression fraction during out-ofhospital cardiac arrest. A prospective alternate month study. Resuscitation. 2015 Aug;93:113-7. doi:

10.1016/j.resuscitation.2015.06.002. PMID: 26070831.

2. Rumball CJ, MacDonald D. The PTL, Combitube, laryngeal mask, and oral airway: a randomized prehospital comparative study of ventilatory device effectiveness and cost-effectiveness in 470 cases of cardiorespiratory arrest. Prehosp Emerg Care. 1997 Jan-Mar;1(1):1-10. doi: 10.1080/10903129708958776. PMID: 9709312.

3. Wang HE, Schmicker RH, Daya MR, et al. Effect of a strategy of initial laryngeal tube insertion vs endotracheal intubation on 72 hour survival in adults with out-of-hospital cardiac arrest: a randomized clinical trial. JAMA. 2018 Aug 28;320(8):769-78. doi: 10.1001/jama.2018.7044. PMID: 30167699 .

4. Chiang WC, Hsieh MJ, Chu HL, et al. The effect of successful intubation on patient outcomes after out-of-hospital cardiac arrest in taipei. Ann Emerg Med. 2018 Mar;71(3):387-96.e2. doi:

10.1016/j.annemergmed.2017.08.008. PMID: 28967516.

5. Evans CC, Petersen A, Meier EN, et al. Prehospital traumatic cardiac arrest: management and outcomes from the resuscitation outcomes consortium epistrytrauma and PROPHET registries. J Trauma Acute Care Surg. 2016 Aug;81(2):285-93. doi: 10.1097/TA.0000000000001070. PMID: 27070438.

6. Hanif MA, Kaji AH, Niemann JT. Advanced airway management does not improve outcome of out-of-hospital cardiac arrest. Acad Emerg Med. 2010 Sep;17(9):926-31. doi: 10.1111/j.15532712.2010.00829.x. PMID: 20836772.
7. Kang K, Kim T, Ro YS, et al. Prehospital endotracheal intubation and survival after out-of-hospital cardiac arrest: results from the Korean nationwide registry. Am J Emerg Med. 2016 Feb;34(2):128-32. doi: 10.1016/j.ajem.2015.09.036. PMID: 26597496.

8. Noda E, Zaitsu A, Hashizume M, et al. Prognosis of patient with cardiopulmonary arrest transported to Kyushu University Hospital. Fukuoka Igaku Zasshi. 2007 Mar;98(3):73-81. PMID: 17461032.

9. Roth D, Hafner C, Aufmesser W, et al. Safety and feasibility of the laryngeal tube when used by EMTs during out-of-hospital cardiac arrest. Am J Emerg Med. 2015 Aug;33(8):1050-5. doi: 10.1016/j.ajem.2015.04.048. PMID: 25957625.

10. Shin SD, Ahn KO, Song KJ, et al. Out-ofhospital airway management and cardiac arrest outcomes: a propensity score matched analysis. Resuscitation. 2012 Mar;83(3):313-9. doi:

10.1016/j.resuscitation.2011.10.028. PMID: 22101202.

11. Sos-Kanto study group. Comparison of arterial blood gases of laryngeal mask airway and bag-valve-mask ventilation in out-of-hospital cardiac arrests. Circ J. 2009 Mar;73(3):490-6. doi: 10.1253/circj.cj-080874. PMID: 19194045.

12. Sulzgruber P, Datler P, Sterz F, et al. The impact of airway strategy on the patient outcome after out-of-hospital cardiac arrest: a propensity score matched analysis. Europ Heart J Acute Cardiovasc Care. 2018 Aug;7(5):423-31. doi: 10.1177/2048872617731894. PMID: 28948850.

13. Takei Y, Enami M, Yachida T, et al. Tracheal intubation by paramedics under limited indication criteria may improve the short-term outcome of out-of-hospital cardiac arrests with noncardiac origin. J Anesth. 2010 Oct;24(5):716-25. doi: 10.1007/s00540-010-0974-6. PMID: 20577765. 
14. Hansen M, Wang H, Le N, et al. Prospective evaluation of airway management in pediatric out-of-hospital cardiac arrest. Resuscitation. 2020 Aug 11;11:11. doi:

10.1016/j.resuscitation.2020.08.003. PMID: 32795596.

15. Hansen ML, Lin A, Eriksson C, et al. A comparison of pediatric airway management techniques during out-ofhospital cardiac arrest using the CARES database. Resuscitation. 2017 Nov;120:516. doi: 10.1016/j.resuscitation.2017.08.015. PMID: 28838781.

16. Hardy GB, Maddry JK, Ng PC, et al. Impact of prehospital airway management on combat mortality. Am J Emerg Med. 2018 Jun;36(6):1032-5. doi: 10.1016/j.ajem.2018.02.007. PMID: 29691106.

17. Ohashi-Fukuda N, Fukuda T, Yahagi N. Effect of pre-hospital advanced airway management for out-of-hospital cardiac arrest caused by respiratory disease: a propensity score-matched study. Anaesth Intensive Care. 2017 May;45(3):375-83. doi: 10.1177/0310057X1704500314. PMID: 28486897.

18. Yanagawa Y, Sakamoto T. Analysis of prehospital care for cardiac arrest in an urban setting in Japan. J Emerg Med. 2010 Apr;38(3):340-5. doi:

10.1016/j.jemermed.2008.04.037. PMID: 18993021 .

19. Fiala A, Lederer W, Neumayr A, et al. EMT-led laryngeal tube vs. face-mask ventilation during cardiopulmonary resuscitation - a multicenter prospective randomized trial. Scand J Trauma Resusc Emerg Med. 2017 Oct 26;25(1):104. doi: 10.1186/s13049-017-0446-1. PMID: 29073915.

20. Chien LC, Hsu HC, Lin CH, et al. Use of an intubating laryngeal mask airway on out-of-hospital cardiac arrest patients in a developing emergency medical service system. J Formos Med Assoc. 2012 Jan;111(1):24-9. doi: 10.1016/j.jfma.2012.01.004. PMID: 22333009 .
21. Nagao T, Kinoshita K, Sakurai A, et al. Effects of bag-mask versus advanced airway ventilation for patients undergoing prolonged cardiopulmonary resuscitation in pre-hospital setting. J Emerg Med. 2012 Feb;42(2):162-70. doi: 10.1016/j.jemermed.2011.02.020. PMID: 22032811 .

22. Jabre P, Penaloza A, Pinero D, et al. Effect of bag-mask ventilation vs endotracheal intubation during cardiopulmonary resuscitation on neurological outcome after out-of-hospital cardiorespiratory arrest: a randomized clinical trial. JAMA. $2018 \mathrm{Feb}$ 27;319(8):779-87. doi:

10.1001/jama.2018.0156. PMID: 29486039.

23. Malinverni S, Bartiaux M, Cavallotto F, et al. Does endotracheal intubation increases chest compression fraction in out of hospital cardiac arrest: a substudy of the CAAM trial. Resuscitation. 2019

Apr;137:35-40. doi:

10.1016/j.resuscitation.2019.01.032. PMID: 30753851.

24. Lupton JR, Schmicker RH, Stephens S, et al. Outcomes with the use of bag-valvemask ventilation during out-of-hospital cardiac arrest in the Pragmatic Airway Resuscitation Trial. Acad Emerg Med. 2020 May;27(5):366-74. doi: 10.1111/acem.13927. PMID: 32220129.

25. Gausche M, Lewis RJ, Stratton SJ, et al. Effect of out-of-hospital pediatric endotracheal intubation on survival and neurological outcome: a controlled clinical trial. see comment erratum appears in JAMA 2000 Jun 28;283(24): 3204. JAMA. 2000;6:783-90. doi: 10.1001/jama.283.6.783. PMID: 10683058.

26. Gausche-Hill M, Lewis RJ, Gunter CS, et al. Design and implementation of a controlled trial of pediatric endotracheal intubation in the out-of-hospital setting. Ann Emerg Med. 2000 Oct;36(4):356-65. doi: 10.1067/mem.2000.109447. PMID: 11020685. 
27. Bernard SA, Nguyen V, Cameron P, et al. Prehospital rapid sequence intubation improves functional outcome for patients with severe traumatic brain injury: a randomized controlled trial. Ann Surg. 2010 Dec;252(6):959-65. doi: 10.1097/SLA.0b013e3181 efc15f. PMID: 21107105.

28. Eckstein M, Chan L, Schneir A, et al. Effect of prehospital advanced life support on outcomes of major trauma patients. J Trauma. 2000 Apr;48(4):643-8. doi: 10.1097/00005373-200004000-00010. PMID: 10780596.

29. Stockinger ZT, McSwain NE, Jr. Prehospital endotracheal intubation for trauma does not improve survival over bag-valve-mask ventilation. J Trauma. 2004 Mar;56(3):531-6. doi: 10.1097/01.ta.0000111755.94642.29. PMID: 15128123.

30. Cooper A, DiScala C, Foltin G, et al. Prehospital endotracheal intubation for severe head injury in children: a reappraisal. Semin Pediatr Surg. 2001 Feb;10(1):3-6. doi: 10.1053/spsu.2001.19379. PMID: 11172563.

31. Yuksen C, Phattharapornjaroen P, Kreethep W, et al. Bag-valve mask versus endotracheal intubation in out-of-hospital cardiac arrest on return of spontaneous circulation: a national database study. Open Access Emerg Med. 2020;12:43-6. doi: 10.2147/OAEM.S229356. PMID: 32210644 .

32. Benger JR, Kirby K, Black S, et al. Effect of a strategy of a supraglottic airway device vs tracheal intubation during outof-hospital cardiac arrest on functional outcome: the AIRWAYS-2 randomized clinical trial. JAMA. 2018 Aug 28;320(8):779-91. doi: 10.1001/jama.2018.11597. PMID: 30167701 .

33. Rabitsch W, Schellongowski P, Staudinger $\mathrm{T}$, et al. Comparison of a conventional tracheal airway with the Combitube in an urban emergency medical services system run by physicians. Resuscitation. 2003 Apr;57(1):27-32. doi: 10.1016/s03009572(02)00435-5. PMID: 12668296.
34. Behrens NH, Fischer M, Krieger T, et al. Effect of airway management strategies during resuscitation from out-of-hospital cardiac arrest on clinical outcome: a registry-based analysis. Resuscitation. 2020 Jul 152:157-64. doi: 10.1016/j.resuscitation.2020.04.015. PMID: 32387124.

35. Kajino K, Iwami T, Kitamura T, et al. Comparison of supraglottic airway versus endotracheal intubation for the pre-hospital treatment of out-of-hospital cardiac arrest. Crit Care. 2011;15(5):R236. doi: 10.1186/cc10483. PMID: 21985431.

36. McMullan J, Gerecht R, Bonomo J, et al. Airway management and out-of-hospital cardiac arrest outcome in the CARES registry. Resuscitation. 2014 May;85(5):617-22. doi: 10.1016/j.resuscitation.2014.02.007. PMID: 24561079.

37. Tanabe $\mathrm{S}$, Ogawa $\mathrm{T}$, Akahane $\mathrm{M}$, et al. Comparison of neurological outcome between tracheal intubation and supraglottic airway device insertion of outof-hospital cardiac arrest patients: a nationwide, population-based, observational study. J Emerg Med. 2013 Feb;44(2):389-97. doi: 10.1016/j.jemermed.2012.02.026. PMID: 22541878.

38. Fukuda T, Sekiguchi H, Taira T, et al. Type of advanced airway and survival after pediatric out-of-hospital cardiac arrest. Resuscitation. 2020 May;150:14553. doi: 10.1016/j.resuscitation.2020.02.005. PMID: 32070779.

39. Davis DP, Peay J, Sise MJ, et al. The impact of prehospital endotracheal intubation on outcome in moderate to severe traumatic brain injury. J Trauma. 2005;58(5):933-9. doi: 10.1097/01.TA.0000162731.53812.58. PMID: 15920406.

40. Wang HE, Szydlo D, Stouffer JA, et al. Endotracheal intubation versus supraglottic airway insertion in out-ofhospital cardiac arrest. Resuscitation. 2012 Sep;83(9):1061-6. doi: 10.1016/j.resuscitation.2012.05.018. PMID: 22664746. 
41. Edwards T, Williams J, Cottee M. Influence of prehospital airway management on neurological outcome in patients transferred to a heart attack centre following out-of-hospital cardiac arrest. Emerg Med Australas. 2019 Feb;31(1):7682. doi: 10.1111/1742-6723.13107. PMID: 29752776.

42. Becker TK, Berning AW, Prabhu A, et al. An assessment of ventilation and perfusion markers in out-of-hospital cardiac arrest patients receiving mechanical CPR with endotracheal or supraglottic airways. Resuscitation. 2018 Jan;122:61-4. doi: 10.1016/j.resuscitation.2017.11.054. PMID: 29175355.

43. Jarman AF, Hopkins CL, Hansen JN, et al. Advanced airway type and its association with chest compression interruptions during out-of-hospital cardiac arrest resuscitation attempts. Prehosp Emerg Care. 2017 Sep-Oct;21(5):628-35. doi: 10.1080/10903127.2017.1308611. PMID: 28459305.

44. Gahan K, Studnek JR, Vandeventer S. King LT-D use by urban basic life support first responders as the primary airway device for out-of-hospital cardiac arrest. Resuscitation. 2011 Dec;82(12):1525-8. doi: 10.1016/j.resuscitation.2011.06.036. PMID: 21756859.

45. Hankins DG, Carruthers N, Frascone RJ, et al. Complication rates for the esophageal obturator airway and endotracheal tube in the prehospital setting. Prehospital Disaster Med. 1993 Apr-Jun;8(2):117-21. doi: 10.1017/s1049023x00040176. PMID: 10148602.

46. Jarvis JL, Wampler D, Wang HE. Association of patient age with first pass success in out-of-hospital advanced airway management. Resuscitation. 2019

Aug;141:136-43. doi:

10.1016/j.resuscitation.2019.06.002.

PMID: 31238034.

47. Frascone RJ, Russi C, Lick C, et al. Comparison of prehospital insertion success rates and time to insertion between standard endotracheal intubation and a supraglottic airway. Resuscitation. 2011 Dec;82(12):1529-36. doi: 10.1016/j.resuscitation.2011.07.009. PMID: 21763247.
48. McCall MJ, Reeves M, Skinner M, et al. Paramedic tracheal intubation using the intubating laryngeal mask airway. Prehosp Emerg Care. 2008 Jan-Mar;12(1):30-4. doi: 10.1080/10903120701709803. PMID: 18189174.

49. Steuerwald MT, Braude DA, Petersen TR, et al. Preliminary report: comparing aspiration rates between prehospital patients managed with extraglottic airway devices and endotracheal intubation. Air Med J. 2018 Jul - Aug;37(4):240-3. doi: 10.1016/j.amj.2018.04.004. PMID: 29935702.

50. Duckett J, Fell P, Han K, et al. Introduction of the I-gel supraglottic airway device for prehospital airway management in a UK ambulance service. Emerg Med J. 2014 Jun;31(6):505-7. doi: 10.1136/emermed-2012-202126. PMID: 23576232.

51. Hiltunen P, Jantti H, Silfvast T, et al. Airway management in out-of-hospital cardiac arrest in Finland: current practices and outcomes. Scand J Trauma Resusc Emerg Med. 2016 Apr 12;24:49. doi: 10.1186/s13049-016-0235-2. PMID: 27071823.

52. Nwanne T, Jarvis J, Barton D, et al. Advanced airway management success rates in a national cohort of emergency medical services agencies. Resuscitation. 2020 Jan 01;146:43-9. doi: 10.1016/j.resuscitation.2019.11.006. PMID: 31756361.

53. Rumball C, Macdonald D, Barber P, et al. Endotracheal intubation and esophageal tracheal Combitube insertion by regular ambulance attendants: a comparative trial. Prehosp Emerg Care. 2004 Jan-

Mar;8(1):15-22. doi: 10.1080/312703002764. PMID: 14691782.

54. McMahan S, Ornato JP, Racht EM, et al. Multi-agency, prehospital evaluation of the pharyngeo-tracheal lumen (PTL) airway. Prehospital Disaster Med. 1992 JanMar;7(1):13-8. doi: 10.1017/s1049023x00039145. PMID: 10149691. 
55. Cady CE, Pirrallo RG. The effect of Combitube use on paramedic experience in endotracheal intubation. Am J Emerg Med. 2005 Nov;23(7):868-71. doi: 10.1016/j.ajem.2005.07.013. PMID: 16291443.

56. Bartlett RL, Martin SD, McMahon JM, Jr., et al. A field comparison of the pharyngeotracheal lumen airway and the endotracheal tube. J Trauma. 1992 Mar;32(3):280-4. doi: 10.1097/00005373199203000-00002. PMID: 1548713.

57. Kwok H, Prekker M, Grabinsky A, et al. Use of rapid sequence intubation predicts improved survival among patients intubated after out-of-hospital cardiac arrest. Resuscitation. 2013

Oct;84(10):1353-8. doi:

10.1016/j.resuscitation.2013.04.015. PMID: 23665389.

58. Cudnik MT, Newgard CD, Daya M, et al. The impact of rapid sequence intubation on trauma patient mortality in attempted prehospital intubation. J Emerg Med. 2010 Feb;38(2):175-81. doi: 10.1016/j.jemermed.2008.01.022. PMID: 18790586.

59. Bendinelli C, Ku D, Nebauer S, et al. A tale of two cities: prehospital intubation with or without paralysing agents for traumatic brain injury. ANZ J Surg. 2018 May;88(5):455-9. doi: 10.1111/ans. 14479. PMID: 29573111.

60. Bulger EM, Copass MK, Sabath DR, et al. The use of neuromuscular blocking agents to facilitate prehospital intubation does not impair outcome after traumatic brain injury. J Trauma. 2005 Apr;58(4):718-23; discussion 23-4. doi:

10.1097/01.ta.0000159239.14181.bc. PMID: 15824647.

61. Vilke GM, Hoyt DB, Epperson M, et al. Intubation techniques in the helicopter. $\mathrm{J}$ Emerg Med. 1994 Mar-Apr;12(2):217-24. doi: 10.1016/0736-4679(94)90702-1. PMID: 8207159.

62. Fouche PF, Smith K, Jennings PA, et al. The association of paramedic rapid sequence intubation and survival in out-ofhospital stroke. Emerg Med J. 2019 Jul;36(7):416-22. doi: 10.1136/emermed2019-208613. PMID: 31147349.
63. Powell EK, Hinckley WR, Stolz U, et al. Predictors of definitive airway sans hypoxia/hypotension on first attempt (DASH-1A) success in traumatically injured patients undergoing prehospital intubation. Prehosp Emerg Care. 2019 Oct 07:1-8. doi:

10.1080/10903127.2019.1670299. PMID: 31539287.

64. Wang HE, Yealy DM. How many attempts are required to accomplish out-of-hospital endotracheal intubation? Acad Emerg Med. 2006 Apr;13(4):372-7. doi: 10.1197/j.aem.2005.11.001. PMID: 16531595 .

65. Prekker ME, Kwok H, Shin J, et al. The process of prehospital airway management: challenges and solutions during paramedic endotracheal intubation. Crit Care Med. 2014 Jun;42(6):1372-8. doi: 10.1097/CCM.0000000000000213. PMID: 24589641.

66. Sunde GA, Heltne JK, Lockey D, et al. Airway management by physician-staffed helicopter emergency medical services - a prospective, multicentre, observational study of 2,327 patients. Scand J Trauma Resusc Emerg Med. 2015 Aug 07;23:57. doi: 10.1186/s13049-015-0136-9. PMID: 26250700 .

67. Eberlein CM, Luther IS, Carpenter TA, et al. First-pass success intubations using video laryngoscopy versus direct laryngoscopy: a retrospective prehospital ambulance service study. Air Med J. 2019 Sep - Oct;38(5):356-8. doi: 10.1016/j.amj.2019.06.004. PMID: 31578974.

68. Myers LA, Gallet CG, Kolb LJ, et al. Determinants of success and failure in prehospital endotracheal intubation. West $\mathrm{J}$ Emerg Med. 2016 Sep;17(5):640-7. doi: 10.5811/westjem.2016.6.29969. PMID: 27625734.

69. Arima T, Nagata O, Miura T, et al. Comparative analysis of airway scope and Macintosh laryngoscope for intubation primarily for cardiac arrest in prehospital setting. Am J Emerg Med. 2014 Jan;32(1):40-3. doi: 10.1016/j.ajem.2013.09.026. PMID: 24176585 . 
70. Ducharme S, Kramer B, Gelbart D, et al. A pilot, prospective, randomized trial of video versus direct laryngoscopy for paramedic endotracheal intubation. Resuscitation. 2017 May;114:121-6. doi: 10.1016/j.resuscitation.2017.03.022. PMID: 28336412.

71. Jarvis JL, McClure SF, Johns D. EMS intubation improves with king vision video laryngoscopy. Prehosp Emerg Care. 2015;19(4):482-9. doi: 10.3109/10903127.2015.1005259. PMID: 25909850 .

72. Risse J, Volberg C, Kratz T, et al. Comparison of videolaryngoscopy and direct laryngoscopy by German paramedics during out-of-hospital cardiopulmonary resuscitation; an observational prospective study. BMC Emerg Med. 2020 Mar 23;20(1):22. doi: 10.1186/s12873-020-00316-z. PMID: 32293276 .

73. Kreutziger J, Hornung S, Harrer C, et al. Comparing the mcgrath mac video laryngoscope and direct laryngoscopy for prehospital emergency intubation in air rescue patients: a multicenter, randomized, controlled trial. Crit Care Med. 2019 Oct;47(10):1362-70. doi: 10.1097/CCM.0000000000003918. PMID: 31389835 .

74. Macke C, Gralla F, Winkelmann M, et al. Increased first pass success with C-MAC videolaryngoscopy in prehospital endotracheal intubation-a randomized controlled trial. J Clin Med. 2020 Aug 22;9(9):22. doi: 10.3390/jcm9092719. PMID: 32842705.

75. Louka A, Stevenson C, Jones G, et al. Intubation success after introduction of a quality assurance program using video laryngoscopy. Air Med J. 2018

Sep;37(5):303-5. doi: 10.1016/j.amj.2018.05.001. PMID: 30322632 .

76. Olvera DJ, Stuhlmiller DFE, Wolfe A, et al. A continuous quality improvement airway program results in sustained increases in intubation success. Prehosp Emerg Care. 2018 Sep-Oct;22(5):602-7. doi: $10.1080 / 10903127.2018 .1433734$. PMID: 29465279.
77. Breeman W, Van Vledder MG, Verhofstad MHJ, et al. First attempt success of video versus direct laryngoscopy for endotracheal intubation by ambulance nurses: a prospective observational study. Eur J Trauma Emerg Surg. 2020 Feb 19;19:19. doi: 10.1007/s00068-020-01326z. PMID: 32072225.

78. Trimmel H, Kreutziger J, Fertsak G, et al. Use of the Airtraq laryngoscope for emergency intubation in the prehospital setting: a randomized control trial. Crit Care Med. 2011 Mar;39(3):489-93. doi: 10.1097/CCM.0b013e318206b69b. PMID: 21169822.

79. Trimmel H, Kreutziger J, Fitzka R, et al. Use of the glidescope ranger video laryngoscope for emergency intubation in the prehospital setting: a randomized control trial. Crit Care Med. 2016 Jul;44(7):e470-6. doi: 10.1097/CCM.0000000000001669. PMID: 27002277.

80. Wayne MA, McDonnell M. Comparison of traditional versus video laryngoscopy in out-of-hospital tracheal intubation. Prehosp Emerg Care. 2010 AprJun;14(2):278-82. doi: 10.3109/10903120903537189. PMID: 20199237.

81. Rocca B, Crosby E, Maloney J, et al. An assessment of paramedic performance during invasive airway management. Prehosp Emerg Care. 2000 AprJun;4(2):164-7. doi: 10.1080/10903120090941443. PMID: 10782606.

82. Chan M, Fehlmann CA, Pasquier M, et al. Endotracheal intubation success rate in an urban, supervised, resident-staffed emergency mobile system: an 11-year retrospective cohort study. J Clin Med. 2020b Jan 16;9(1):16. doi: 10.3390/jcm9010238. PMID: 31963162.

83. Hossfeld B, Thierbach S, Allgoewer A, et al. First pass success of tracheal intubation using the C-MAC PM videolaryngoscope as first-line device in prehospital cardiac arrest compared with other emergencies: an observational study. Eur J Anaesthesiol. 2020 Aug 21;21:21. doi: 10.1097/EJA.0000000000001286. PMID: 32833853 . 
84. Gellerfors M, Larsson A, Svensen CH, et al. Use of the Airtraq device for airway management in the prehospital setting--a retrospective study. Scand J Trauma Resusc Emerg Med. 2014 Feb 03;22:10. doi: 10.1186/1757-7241-22-10. PMID: 24484856.
85. Delorenzo A, St Clair T, Andrew E, et al. Prehospital rapid sequence intubation by intensive care flight paramedics. Prehosp Emerg Care. 2018 Sep-Oct;22(5):595-601. doi: 10.1080/10903127.2018.1426666.

PMID: 29405803. 